\title{
Streamlined Catalytic Enantioselective Synthesis of $\alpha$-Substituted $\beta, \gamma$-Unsaturated Ketones and Either of the Corresponding Tertiary Homoallylic Alcohol Diastereomers
}

\author{
Juan del Pozo, ${ }^{1}$ Shaochen Zhang, ${ }^{1}$ Filippo Romiti, ${ }^{1,2}$ Shibo Xu, ${ }^{1}$ Ryan P. Conger ${ }^{1}$ and Amir H. Hoveyda ${ }^{1,2}$ \\ ${ }^{1}$ Department of Chemistry, Merkert Chemistry Center, Boston College, Chestnut Hill, Massachusetts 02467, \\ USA \\ ${ }^{2}$ Supramolecular Science and Engineering Institute, University of Strasbourg, CNRS, 67000 Strasbourg, France \\ e-mail: amir.hoveyda@bc.edu or ahoveyda@unistra.fr
}

\section{SUPPORTING INFORMATION \\ Part One: Experimentals \& Analytical Data}

\section{Table of Content}

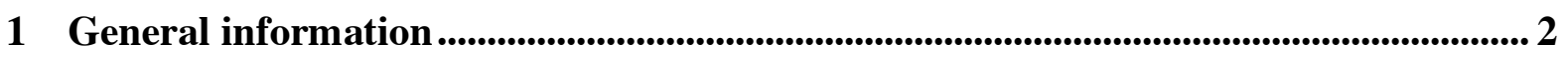

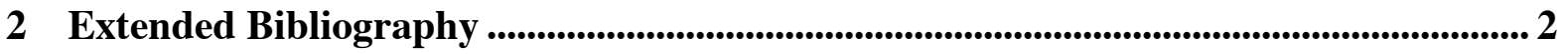

2.1 Selected reviews on catalytic enantioselective synthesis of tertiary alcohols ........................2

2.2 Selected reviews on catalytic enantioselective allyl and crotyl additions to carbonyl-containing compounds 3

2.3 Selected enantioselective allyl additions to carbonyl-containing compounds with Ag-, Ti-, Cr-, Cu-based catalysts

2.4 Enantioselective allyl additions to carbonyl-containing compounds with non-metal-based

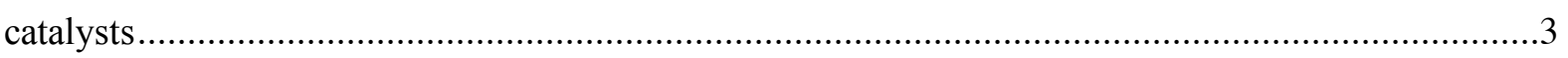

2.5 Enantioselective reductive coupling of ketones and allyl acetate with Ir-based catalysts ........4

2.6 Proline-catalyzed Mannich reactions with $\alpha$-hydroxy ketones .............................................4

2.7 Enantioselective synthesis of $\alpha$-Hydroxy aryl ketones, cyclic ketones and $\beta$-ketoesters..........4

2.8 Synthesis of $\alpha$-hydroxy ketones and derivatives by catalytic enantioselective allylic substitution .5

2.9 Catalytic enantioselective dihydroxyl additions to enol ethers derived from ketones ..............5

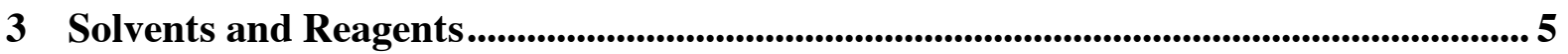

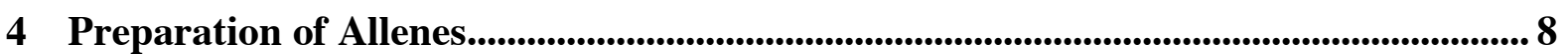


4.1 Through Crabbé processes

4.1.1 $t$-Butyldimethyl(penta-3,4-dien-1-yloxy)silane ...........................................

4.1.2 Methyl hexa-4,5-dienoate......................................................................... 8

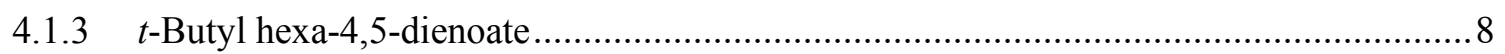

4.1.4 Methyl buta-2,3-dien-1-ylcarbamate .....................................................

4.1.5 $t$-Butyl buta-2,3-dien-1-ylcarbamate......................................................... 8

4.1.6 Penta-3,4-dien-1-yl ( $t$-butoxycarbonyl)-L-valinate ............................................

4.1.7 Propa-1,2-dien-1-ylbenzene ...............................................................

Through allylic substitutions .........................................................................

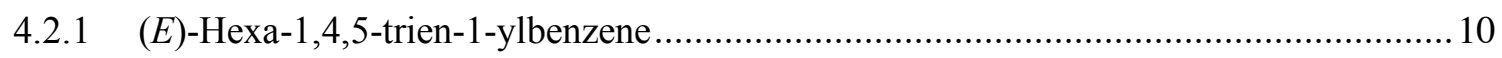

Through propargylic substitutions ................................................................... 10

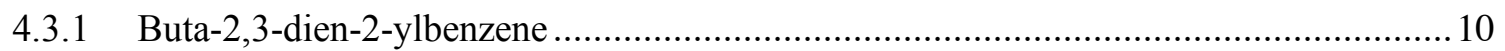

4.3.2 $t$-Butyldimethyl((2-methylbuta-2,3-dien-1-yl)oxy)silane .................................. 10

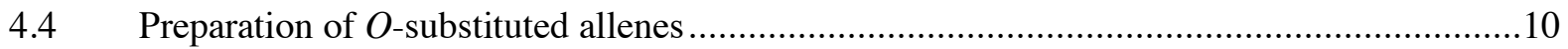

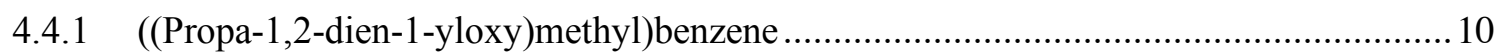

4.4.2 1-Methoxy-4-((propa-1,2-dien-1-yloxy)methyl)benzene...................................... 10

4.4.3 $t$-Butyldimethyl((2-methylbuta-2,3-dien-1-yl)oxy)silane ................................. 11

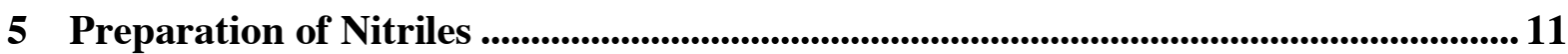

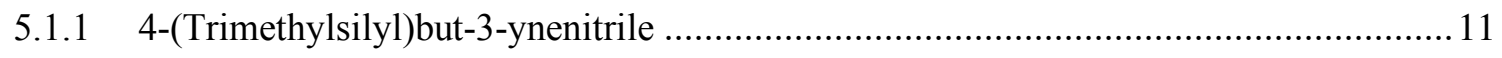

6 Synthesis of $\beta, \gamma$-unsaturated ketones ........................................................................ 11

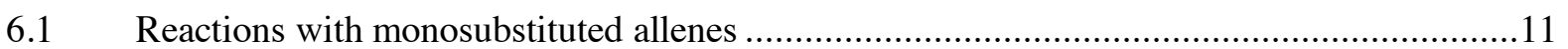

6.2 Reactions with aryl and heteroaryl nitriles....................................................... 12

6.2.1 (R)-Methyl 5-(4,4,5,5-tetramethyl-1,3,2-dioxaborolan-2-yl)-4-(2-(trifluoromethyl) benzoyl)hex-5-enoate $(2 \mathrm{a})$

6.2.2 Methyl (S)-(3-(4,4,5,5-tetramethyl-1,3,2-dioxaborolan-2-yl)-2-(4-(4,4,5,5-tetrame-thyl1,3,2-dioxaborolan-2-yl)benzoyl)but-3-en-1-yl)carbamate (2b)....

6.2.3 (R)-1-(4-Bromophenyl)-2-methyl-3-(4,4,5,5-tetramethyl-1,3,2-dioxaborolan-2-yl)but-3-

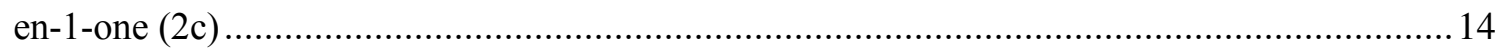

6.2.4 t-Butyl (R)-4-(4-aminobenzoyl)-5-(4,4,5,5-tetramethyl-1,3,2-dioxaborolan-2-yl) hex-5enoate $(2 \mathrm{~d})$.

6.2.5 (R)-1-(Benzofuran-2-yl)-2-(2-((t-butyldimethylsilyl)oxy)ethyl)-3-(4,4,5,5-tetramethyl1,3,2-dioxaborolan-2-yl)but-3-en-1-one (2e). 
6.2.6 $t$-Butyl-(R)-5-(2-(2-((t-butyldimethylsilyl)oxy)ethyl)-3-(4,4,5,5-tetramethyl-1,3,2dioxaborolan-2-yl)but-3-enoyl)-1H-indole-1-carboxylate (2f)

6.2.7 (R)-2-(2-((t-Butyldimethylsilyl)oxy)ethyl)-1-(pyridin-3-yl)-3-(4,4,5,5-tetramethyl-1,3,2dioxaborolan-2-yl)but-3-en-1-one (2g)

6.2.8 (S)-2-(2-((t-Butyldimethylsilyl)oxy)ethyl)-3-(4,4,5,5-tetramethyl-1,3,2-dioxaborolan-2yl)-1-(thiophen-2-yl)but-3-en-1-one (2h)

6.2.9 (S)-1,2-Diphenyl-3-(4,4,5,5-tetramethyl-1,3,2-dioxaborolan-2-yl)but-3-en-1-one (2i).. 18 6.2.10 (R)-4-(2-Methyl-3-(4,4,5,5-tetramethyl-1,3,2-dioxaborolan-2-yl)but-3enoyl)benzenesulfonyl fluoride $(2 \mathrm{j})$.

6.2.11 (R)-2-(2-((t-Butyldimethylsilyl)oxy)ethyl)-1-phenyl-3-(4,4,5,5-tetramethyl-1,3,2dioxaborolan-2-yl)but-3-en-1-one (2k)

6.2.12 (R)-2-Methyl-1-phenyl-3-(4,4,5,5-tetramethyl-1,3,2-dioxaborolan-2-yl)but-3-en-1one (21)

6.2.13 (R)-1-(4-Fluorophenyl)-2-methyl-3-(4,4,5,5-tetramethyl-1,3,2-dioxaborolan-2-yl)but-

3 -en-1-one (2m; precursor to $[(S, R)-8 \mathrm{~d}]$; see Fig. $3 \mathrm{c})$ 21

6.3 Reactions with $\alpha, \beta$-unsaturated nitriles .22

6.3.1 (R,E)-3-(2-((t-Butyldimethylsilyl)oxy)ethyl)-2-(4,4,5,5-tetramethyl-1,3,2-dioxaborolan2-yl)nona-1,5-dien-4-one (3a)......

6.3.2 $t$-Butyl $\quad(R, Z)-4-(4-(2-((t$-butyldimethylsilyl)oxy)ethyl)-3-oxo-5-(4,4,5,5-tetramethyl1,3,2-dioxaborolan-2-yl)hexa-1,5-dien-1-yl)piperidine-1-carboxylate (3b).

6.3.3 (R,E)-2-Methyl-7-phenyl-4-(1-(4,4,5,5-tetramethyl-1,3,2-dioxaborolan-2-yl)vinyl)hepta1,6-dien-3-one (3c).

6.4.1 (R)-7-Bromo-3-(2-((t-butyldimethylsilyl)oxy)ethyl)-2-(4,4,5,5-tetramethyl-1,3,2dioxaborolan-2-yl)hept-1-en-4-one (4a)

6.4.2 (R)-3-(2-((t-Butyldimethylsilyl)oxy)ethyl)-2-(4,4,5,5-tetramethyl-1,3,2-dioxaborolan-2yl)nona-1,8-dien-4-one (4b).

6.4.3 (R)-3-Cyclohexyl-2-(4,4,5,5-tetramethyl-1,3,2-dioxaborolan-2-yl)non-1-en-8-yn-4-one $(4 \mathrm{c})$

6.4.4 Methyl (R)-5-(2-((t-butyldimethylsilyl)oxy)ethyl)-4-oxo-6-(4,4,5,5-tetramethyl-1,3,2dioxaborolan-2-yl)hept-6-enoate (4d)

6.4.5 (R,E)-10-Azido-1-phenyl-4-(1-(4,4,5,5-tetramethyl-1,3,2-dioxaborolan-2-yl)vinyl)dec1-en-5-one (4e) .28

6.4.6 $t$-Butyl $\quad(R)-5$-oxo-10-(4-phenyl-1H-1,2,3-triazol-1-yl)-4-(1-(4,4,5,5-tetramethyl-1,3,2dioxaborolan-2-yl)vinyl)decanoate (4f). 
6.4.7 (R)-3-(2-((t-Butyldimethylsilyl)oxy)ethyl)-4-(4,4,5,5-tetramethyl-1,3,2-dioxaborolan-2yl)pent-4-en-2-one (4g)

6.4.8 $t$-Butyl $\quad(S)$-(2-acetyl-3-(4,4,5,5-tetramethyl-1,3,2-dioxaborolan-2-yl)but-3-en-1yl)carbamate (4h) 31

6.4.9 (S)-3-Phenyl-4-(4,4,5,5-tetramethyl-1,3,2-dioxaborolan-2-yl)pent-4-en-2-one (4i) ......31

6.4.10 (R)-3-(2-Fluoroacetyl)-4-(4,4,5,5-tetramethyl-1,3,2-dioxaborolan-2-yl)pent-4-en-1-yl ( $t$-butoxycarbonyl)-L-valinate $(4 \mathrm{j})$

6.4.11 (S)-3-(2-Fluoroacetyl)-4-(4,4,5,5-tetramethyl-1,3,2-dioxaborolan-2-yl)pent-4-en-1-yl ( $t$-butoxycarbonyl)-L-valinate $(4 \mathrm{k})$

6.4.12 $t$-Butyl $\quad(S)$-(4,4-dimethyl-3-oxo-2-(1-(4,4,5,5-tetramethyl-1,3,2-dioxaborolan-2yl)vinyl)pentyl)carbamate (4l)

6.4.13 (R)-3-(2-((t-Butyldimethylsilyl)oxy)ethyl)-2-(4,4,5,5-tetramethyl-1,3,2-dioxaborolan2-yl)hepta-1,6-dien-4-one (4m).....

6.4.14 $(R)-3-(2-((t$-Butyldimethylsilyl)oxy)ethyl)-2-(4,4,5,5-tetramethyl-1,3,2-dioxaborolan2-yl)-7-(trimethylsilyl)hept-1-en-6-yn-4-one (4n) 35

6.4.15 $t$-Butyl $(R)-4-(2-((t$-butyldimethylsilyl)oxy)ethyl)-3-oxo-5-(4,4,5,5-tetramethyl-1,3,2dioxaborolan-2-yl)hex-5-enoate $(4 \mathrm{o})$

6.4.16 (2R,4R)-4-(2-((t-Butyldimethylsilyl)oxy)ethyl)-2-phenyl-5-(4,4,5,5-tetramethyl-1,3,2dioxaborolan-2-yl)hex-5-en-3-one (4p).

6.4.17 $(2 S, 4 R)-4-(2-((t-B u t y l d i m e t h y l s i l y l) o x y) e t h y l)-2-p h e n y l-5-(4,4,5,5-t e t r a m e t h y l-1,3,2-$ dioxaborolan-2-yl)hex-5-en-3-one (4q).

6.5.1 (R)-2-(((t-Butyldimethylsilyl)oxy)methyl)-2-methyl-1-phenyl-3-(4,4,5,5-tetramethyl1,3,2-dioxaborolan-2-yl)but-3-en-1-one (5a).

6.5.2 (R)-1-(Benzo[ $d][1,3]$ dioxol-5-yl)-2-(((t-butyldimethylsilyl)oxy)methyl)-2-methyl-3(4,4,5,5-tetramethyl-1,3,2-dioxaborolan-2-yl)but-3-en-1-one (5b).....

6.5.3 $t$-Butyl-(R)-3-(2-(((t-butyldimethylsilyl)oxy)methyl)-2-methyl-3-(4,4,5,5-tetramethyl1,3,2-dioxaborolan-2-yl)but-3-enoyl)- $1 H$-indole-1-carboxylate ( $5 \mathrm{c})$. 40

6.5.4 (R)-3-Methyl-3-phenyl-4-(4,4,5,5-tetramethyl-1,3,2-dioxaborolan-2-yl)pent-4-en-2-one

6.5.5 (R)-1-Cyclohexyl-2-methyl-2-phenyl-3-(4,4,5,5-tetramethyl-1,3,2-dioxaborolan-2yl)but-3-en-1-one (5e)

6.5.6 (R)-4-Methyl-4-phenyl-5-(4,4,5,5-tetramethyl-1,3,2-dioxaborolan-2-yl)-1(trimethylsilyl)hex-5-en-1-yn-3-one (5f)

6.5.7 (R)-2-Methyl-1,2-diphenyl-3-(4,4,5,5-tetramethyl-1,3,2-dioxaborolan-2-yl)but-3-en-1one $(5 \mathrm{~g})$ 
6.6 Reactions with $O$-substituted allenes .45

6.6.1 (R)-1-Phenyl-3-(4,4,5,5-tetramethyl-1,3,2-dioxaborolan-2-yl)-2-

((triisopropylsilyl)oxy)but-3-en-1-one (6a)

6.6.2 (R)-3-(Benzyloxy)-2-(4,4,5,5-tetramethyl-1,3,2-dioxaborolan-2-yl)non-1-en-8-yn-4-one

6.6.3 (R)-3-(Benzyloxy)-2-(4,4,5,5-tetramethyl-1,3,2-dioxaborolan-2-yl)hepta-1,6-dien-4-one

6.6.4 Methyl (R)-5-(benzyloxy)-4-oxo-6-(4,4,5,5-tetramethyl-1,3,2-dioxaborolan-2-yl)hept-6enoate $(6 \mathrm{~d})$

6.6.5 (R)-4-(Benzyloxy)-5-(4,4,5,5-tetramethyl-1,3,2-dioxaborolan-2-yl)hex-5-en-3-one (6e) ..

6.6.6 (R)-3-((4-Methoxybenzyl)oxy)-1-phenyl-4-(4,4,5,5-tetramethyl-1,3,2-dioxaborolan-2-

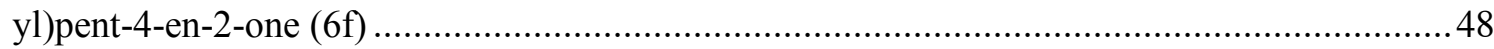

\section{Studies Regarding Mechanism and Variations in Conditions.......................................49}

7.1 Impact of time on enantioselectivity in a reaction of a nitrile with an electron-deficient substituent .... .49

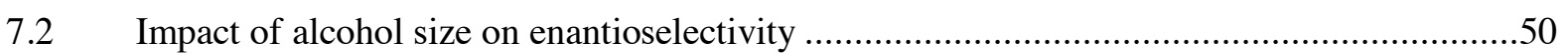

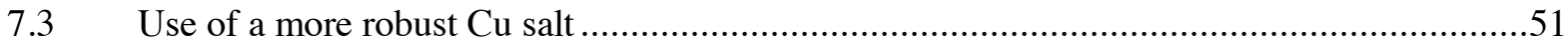

7.4 The optimal conditions for reactions involving 1 ,1-disubstituted allenes ..........................51

7.5 The impact of ketimine hydrolysis conditions on product enantioselectivity .....................52

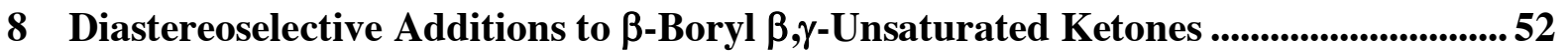

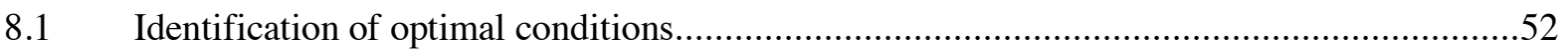

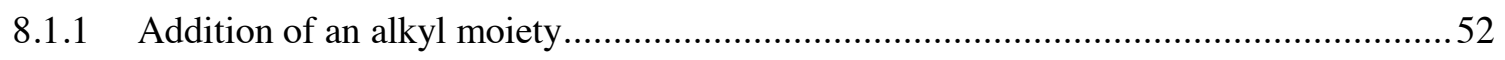

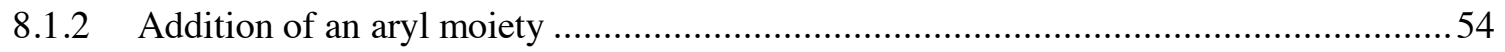

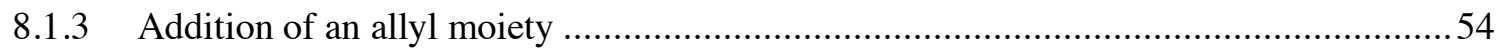

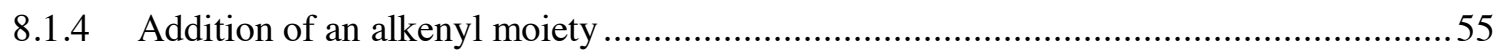

8.1.5 Regarding boronic acid products ..............................................................5

8.2 Procedures for Addition of an Organometallic Compounds to a $\beta, \gamma$-Unsaturated Ketone .....56

8.2.1 General procedure for addition of an organometallic compound based on $\mathrm{CeCl}_{3} \cdot 2 \mathrm{LiCl} 156$

8.2.2 For addition of an organolithium compound (no additive) ...................................56

8.2.3 For addition of a Grignard reagent to an $\alpha$-substituted OPMB ketone .......................57

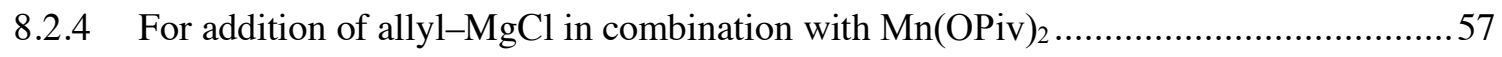

8.2.5 For addition of an allylmagnesium halide compound to an $\alpha$-substituted OPMB ketone .. 


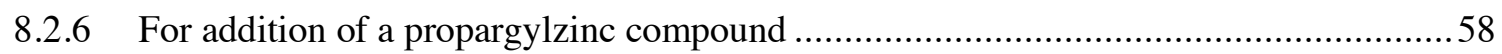

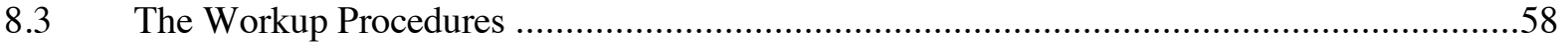

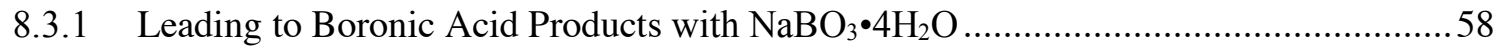

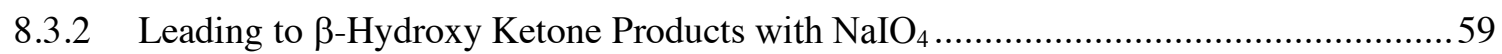

8.4 Regarding possible loss of enantiomeric purity upon nucleophilic addition to an $\alpha$-substituted ketone .59

8.5 Analytical Data for Tertiary Alcohols

8.5.1 ((3R,4R)-3-(2-((t-Butyldimethylsilyl)oxy)ethyl)-4-hydroxy-4-phenylpent-1-en-2yl)boronic acid $[(R, R)-7 \mathrm{a}]$

8.5.2 ((3R,4S)-3-(2-((t-Butyldimethylsilyl)oxy)ethyl)-4-hydroxy-4-phenylpent-1-en-2yl)boronic acid $[(S, R)-7 \mathrm{a}]$

8.5.3 ((3R,4S)-3-(2-((t-Butyldimethylsilyl)oxy)ethyl)-4-hydroxy-4-methylhepta-1,6-dien-2yl)boronic acid (7b)

8.5.4 ((3R,4S)-3-(2-((t-Butyldimethylsilyl)oxy)ethyl)-4-hydroxy-4,5,5-trimethylhex-1-en-2yl)boronic acid $(7 \mathrm{c})$

8.5.5 (3S,4S)-4-Hydroxy-3-((4-methoxybenzyl)oxy)-4-methyl-5-phenylpentan-2-one (7d) ..62

8.5.6 ((3R,4S)-3-(2-((t-butyldimethylsilyl)oxy)ethyl)-4-hydroxy-4-phenylhepta-1,6-dien-2yl)boronic acid $[(S, R)-8 \mathrm{a}]$

8.5.7 ((3R,4R)-3-(2-((t-Butyldimethylsilyl)oxy)ethyl)-4-hydroxy-4-phenylhepta-1,6-dien-2yl)boronic acid $[(R, R)-8 \mathrm{a}]$

8.5.8 (3S,4S)-3-(2-((t-Butyldimethylsilyl)oxy)ethyl)-4-hydroxy-4-(pyridin-2-yl)pentan-2-one

8.5.9 (3S,4R)-4-hydroxy-3-((4-methoxybenzyl)oxy)-5-phenyl-4-(thiophen-3-yl)pentan-2-one

8.5.10 ((3R,4S)-4-(4-Fluorophenyl)-4-hydroxy-3-methyl-4-phenylbut-1-en-2-yl)boronic acid $[(S, R)-8 \mathrm{~d}]$

8.5.11 ((3R,4R)-4-(4-Fluorophenyl)-4-hydroxy-3-methyl-4-phenylbut-1-en-2-yl)boronic acid $[(R, R)-8 \mathrm{~d}]$ .66

8.5.12 ((3R,4R)-4-Hydroxy-3-methyl-4-phenylhepta-1,6-dien-2-yl)boronic acid (9a). 67

8.5.13 ((3S,4R)-3-(((t-Butoxycarbonyl)amino)methyl)-4-hydroxy-4-methylhepta-1,6-dien-2yl)boronic acid (9b)

8.5.14 (3R,4S)-4-hydroxy-3,4-dimethyl-3-phenylhepta-1,6-dien-2-yl)boronic acid (9c) ....68

8.5.15 (3S,4S)-4-Benzyl-4-hydroxy-3-((4-methoxybenzyl)oxy)hept-6-en-2-one (9d) .........69 
8.5.16 (R)-5-Allyl-5-((R)-5-((t-butyldimethylsilyl)oxy)-2-(4,4,5,5-tetramethyl-1,3,2dioxaborolan-2-yl)pent-1-en-3-yl)dihydrofuran-2(3H)-one (9e; intermediate for $4 \mathrm{~d} \rightarrow 13$, Scheme 12)

8.5.17 ((3R,4R)-3-(2-((t-Butyldimethylsilyl)oxy)ethyl)-4-hydroxy-4-phenylhexa-1,5-dien-2yl)boronic acid (10)

8.5.18 ((3R,4S)-3-(2-((t-Butyldimethylsilyl)oxy)ethyl)-4-hydroxy-4(trimethylsilyl)ethynyl)hepta-1,6-dien-2-yl)boronic acid (11)

8.5.19 (3S,4R)-3-(2-((t-butyldimethylsilyl)oxy)ethyl)-4-hydroxy-4-methyl-7-

(trimethylsilyl)hept-6-yn-2-one (12a).

8.5.20 (R)-5-((S)-1-((t-butyldimethylsilyl)oxy)-4-oxopentan-3-yl)-5-(3-(trimethylsilyl)prop-

2-yn-1-yl)dihydrofuran-2(3H)-one (12b) .72

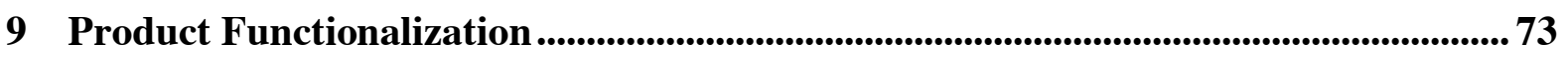

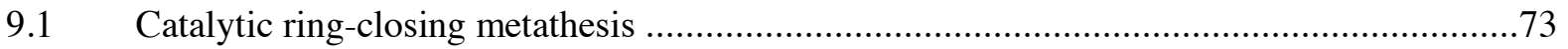

9.1.1 (5R,6R)-6-(2-((t-Butyldimethylsilyl)oxy)ethyl)-7-(4,4,5,5-tetramethyl-1,3,2-

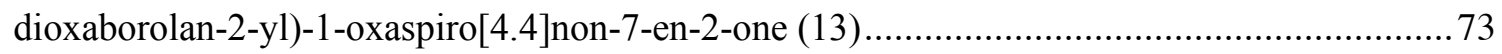

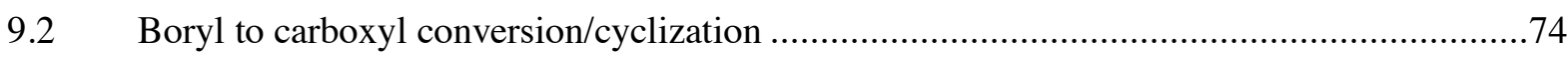

9.2.1 (4R,5S)-5-(4-Fluorop.enyl)-4-methyl-3-methylene-5-phenyldihydrofuran-2(3H)-one (14) .74

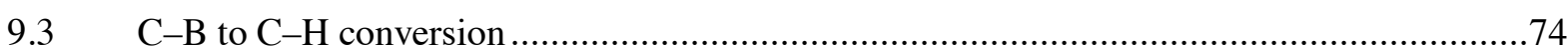

9.3.1 (3S,4R)-4-(2-((t-Butyldimethylsilyl)oxy)ethyl)-2,2,3-trimethylhex-5-en-3-ol (15a)......74

9.3.2 (1S,2R)-1-(4-Fluorophenyl)-2-methyl-1-phenylbut-3-en-1-ol (15b) ….........................75

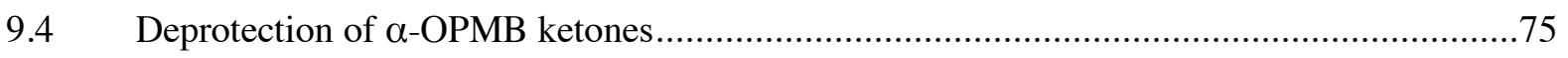

9.4.1 (R)-3-Hydroxy-1-phenyl-4-(4,4,5,5-tetramethyl-1,3,2-dioxaborolan-2-yl)pent-4-en-2-

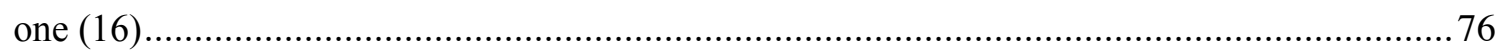

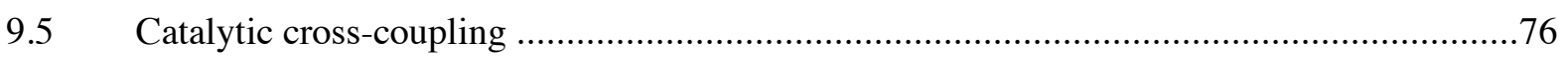

9.5.1 (3R,4R)-3-(2-((t-Butyldimethylsilyl)oxy)ethyl)-4-phenyl-2-(pyridin-2-yl)hepta-1,6-dien-

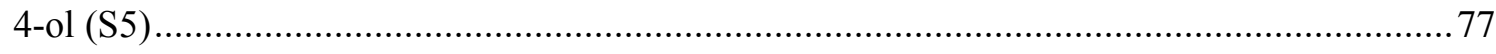

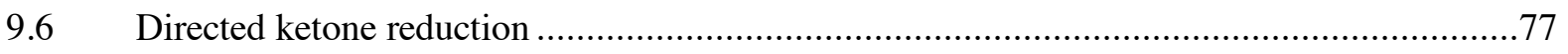

9.6.1 (2S,3R,4R)-3-Methyl-4-phenylhept-6-ene-2,4-diol (S7) ........................................... 78

10 Synthesis of the Bicyclic Fragments of (+)-Rubriflordilactone A and B, and 5-epi-

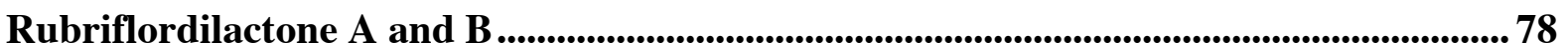

10.1 Synthesis of the Bicyclic Fragments of (+)-Rubriflordilactone A and B ..............................78

10.1.1 (S)-3-(2-((t-Butyldimethylsilyl)oxy)ethyl)-2-(4,4,5,5-tetramethyl-1,3,2-dioxaborolan2-yl)hepta-1,6-dien-4-one ( $S$-4m) 78 
10.1.2 (3R,4R)-3-(2-((t-Butyldimethylsilyl)oxy)ethyl)-4-(3,3-diethoxypropyl)-4hydroxyhept-6-en-2-one (18)

10.1.3 (R)-5-Allyl-5-((R)-1-((t-butyldimethylsilyl)oxy)-4-oxopentan-3-yl)dihydrofuran2(3H)-one (19)

10.1.4 (R)-5-Allyl-5-((S)-1-((t-butyldimethylsilyl)oxy)-4-hydroxy-4-methylpentan-3yl)dihydrofuran-2(3H)-one (20)

10.1.5 (3aR,6S,6aR)-6a-Allyl-6-(2-((t-butyldimethylsilyl)oxy)ethyl)-5,5dimethyltetrahydrofuro[3,2-b]furan-2(3H)-one (21)

10.1.6 (3aR,6S,6aR)-6-(2-((t-Butyldimethylsilyl)oxy)ethyl)-5,5-dimethyl-6a-(prop-2-yn-1yl)tetrahydrofuro[3,2-b]furan-2(3H)-one (22)

10.1.7 2-((3S,3a R,6aR)-2,2-Dimethyl-5-oxo-3a-(prop-2-yn-1-yl)hexahydrofuro[3,2-b]furan-

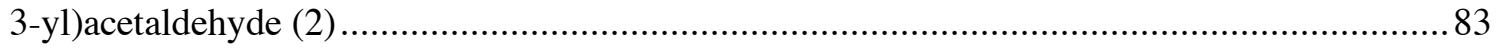

10.2 Synthesis of bicyclic fragment of 5-epi-rubriflordilactone A and B .85

10.2.1 (R)-5-((R)-1-((t-Butyldimethylsilyl)oxy)-4-hydroxy-4-methylpentan-3-yl)-5-(3-

(trimethylsilyl)prop-2-yn-1-yl)dihydrofuran-2(3H)-one (23)

10.2.2 (3aR,6R,6a $R)-6$-(2-Hydroxyethyl)-5,5-dimethyl-6a-(prop-2-yn-1-

yl)tetrahydrofuro[3,2-b]furan-2(3H)-one (S10)

10.2.3 2-((3R,3a $R, 6 \mathrm{a} R)$-2,2-Dimethyl-5-oxo-3a-(prop-2-yn-1-yl)hexahydrofuro[3,2-b]furan-

3-yl)acetaldehyde (diast-2)

11 Determination of the absolute and relative configuration of products 87

11.1.1 (4R,5R,6S)-4-allyl-2,2,5,6-tetramethyl-4-phenyl-1,3-dioxane (S12) .88

12 X-ray Structures

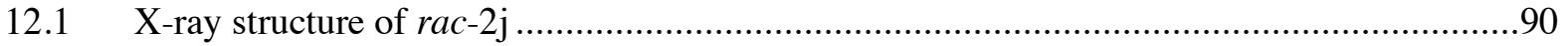

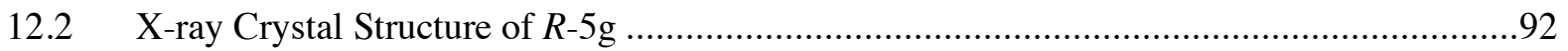

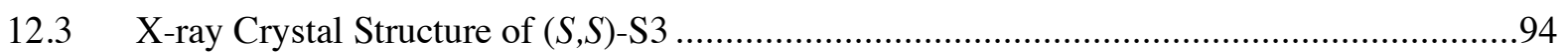

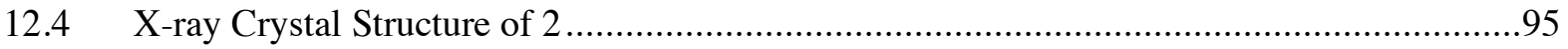




\section{General information}

Infrared (IR) spectra were recorded on a Bruker FT-IR Alpha (ATR mode) spectrophotometer, $\lambda_{\max }$ in $\mathrm{cm}^{-1}$. Bands are characterized as broad (br), strong (s), medium (m), and weak (w). ${ }^{1} \mathrm{H}$ NMR spectra were recorded on a Varian Unity INOVA 400 (400 MHz), Varian Unity INOVA $500(500 \mathrm{MHz})$, or Varian Unity INOVA $600(600 \mathrm{MHz})$ spectrometer. Chemical shifts are reported in ppm from tetramethylsilane with the solvent resonance as the internal standard $\left(\mathrm{CDCl}_{3}: \delta 7.26 \mathrm{ppm}\right)$. Data are reported as follows: chemical shift, integration, multiplicity (s $=$ singlet, $\mathrm{d}=$ doublet, $\mathrm{t}=$ triplet, $\mathrm{q}=$ quartet, $\mathrm{br}=$ broad, $\mathrm{m}=$ multiplet $)$, and coupling constant (Hz). 13C NMR spectra were recorded on a Varian Unity INOVA 400 (101 MHz), Varian Unity INOVA $500(126 \mathrm{MHz})$ or Varian Unity INOVA $600(151 \mathrm{MHz})$ spectrometer with complete proton decoupling. Chemical shifts are reported in ppm from tetramethylsilane with the solvent resonance as the internal standard $\left(\mathrm{CDCl}_{3}: \delta 77.16 \mathrm{ppm}\right) .{ }^{11} \mathrm{~B}$ NMR spectra were recorded on a Varian Unity INOVA $400(128 \mathrm{MHz})$, Varian Unity INOVA $500(160 \mathrm{MHz})$ or Varian Unity INOVA $600(192 \mathrm{MHz})$ spectrometer with complete proton decoupling. Chemical shifts are reported in ppm with $\mathrm{BF}_{3} \cdot \mathrm{Et}_{2} \mathrm{O}\left(10 \%\right.$ in $\left.\mathrm{CDCl}_{3}\right)$ as reference. Highresolution mass spectrometry was performed on a JEOL AccuTOF DART (positive mode) at the Mass Spectrometry Facility, Boston College. Enantiomeric ratios were determined by HPLC analysis KROMASIL $(R, R)$-WHELK-O1 (4.6 x $250 \mathrm{~mm})$, Chiral Technologies Chiralpak AZ-H (4.6 x $250 \mathrm{~mm})$, Chiralcel OD-H (4.6 x $250 \mathrm{~mm})$, Chiralpak AD-H (4.6 x $250 \mathrm{~mm})$, Chiralcel OZ-H (4.6 x $250 \mathrm{~mm})$ and Chiralpak IC $(4.6 \times 250 \mathrm{~mm}))$ in comparison with authentic racemic materials. Specific rotations were measured on an ATAGO ${ }^{\circledR}$ AP-300 Automatic Polarimeter or a Rudolph Research Analytical Autopol IV Polarimeter. Melting points were measured on a Thomas Hoover capillary melting point apparatus and are uncorrected.

Unless noted otherwise, reactions were carried out with distilled and degassed solvents under an atmosphere of dry $\mathrm{N}_{2}$ in oven- $\left(135^{\circ} \mathrm{C}\right)$ or flame-dried glassware with standard glovebox or vacuum-line techniques. Solvents were purified under a positive pressure of dry argon by a modified Innovative Technologies purification system: toluene, benzene and hexanes were purified through a copper oxide column and an alumina column; $\mathrm{CH}_{2} \mathrm{Cl}_{2}$ and $\mathrm{Et}_{2} \mathrm{O}$ were purged with argon and purified by passage through two alumina columns. Tetrahydrofuran (THF; Sigma-Aldrich) was purified by distillation from sodium benzophenone ketyl immediately prior to use unless specified otherwise. Work-up and purification procedures involved the use of reagent grade solvents (Fisher Scientific) in air.

\section{Extended Bibliography}

\subsection{Selected reviews on catalytic enantioselective synthesis of tertiary alcohols}

a) Hatano, M.; Ishihara, K. Recent progress in the catalytic synthesis of tertiary alcohols from ketones with organometallic reagents. Synthesis, 2008, 1647-1675. 
b) Shibasaki, M.; Kanai, M. Asymmetric synthesis of tertiary alcohols and $\alpha$-tertiary amines via $\mathrm{Cu}$-catalyzed $\mathrm{C}-\mathrm{C}$ bond formation to ketones and ketimines. Chem. Rev. 2008, 108, 2853-2873 (2008).

2.2 Selected reviews on catalytic enantioselective allyl and crotyl additions to carbonylcontaining compounds

a) Denmark, S. E.; Fu, J. Catalytic enantioselective addition of allylic organometallic reagents to aldehydes and ketones. Chem. Rev. 2003, 103, 2763-2794.

b) Yus, M.; González-Gómez, J. C.; Foubelo, F. Catalytic enantioselective allylation of carbonyl compounds and imines. Chem. Rev. 2011, 111, 7774-7854.

2.3 Selected enantioselective allyl additions to carbonyl-containing compounds with Ag-, Ti-, Cr-, Cu-based catalysts

a) Casolari, S.; D’Addari, D.; Tagliavini, E. BINOL-Ti-catalyzed synthesis of tertiary homoallylic alcohols: the first catalytic asymmetric allylation of ketones. Org. Lett. 1999, 1, 1061-1063.

b) Hanawa, H.; Kii, S.; Maruoka, K. New chiral bis-titanium(IV) catalyst with dibenzofuran spacer for catalytic asymmetric allylation of aldehydes and aryl ketones. Adv. Synth. Catal. 2001, 343, 57-60.

c) Wadamoto, M.; Yamamoto, H. Silver-catalyzed asymmetric Sakurai-Hosomi allylation of ketones. J. Am. Chem. Soc. 2005, 127, 14556-14557.

d) Teo, Y.-C.; Goh, J.-D.; Loh, T.-P. Catalytic enantioselective allylation of ketones via a chiral indium(III) complex. Org. Lett. 2005, 7, 2743-2745.

e) Haddad, T. D.; Hirayama, L. C.; Singaram, B. Indium-mediated asymmetric Barbiertype allylations: Additions to aldehydes and ketones and mechanistic investigation of the organoindium reagents. J. Org. Chem. 2010, 75, 642-649.

f) Miller, J. J.; Sigman, M. S. Design and synthesis of modular oxazoline ligands for the enantioselective chromium-catalyzed addition of allyl bromide to ketones. J. Am. Chem. Soc. 2007, 129, 2752-2753.

g) Wada, R.; Oisaki, K.; Kanai, M.; Shibasaki, M. Catalytic enantioselective allylboration of ketones. J. Am. Chem. Soc. 2004, 126, 8910-8911.

h) Shi, S. L.; Xu, L. W.; Oisaki, K.; Kanai, M.; Shibasaki, M. Identification of modular chiral bisphosphines effective for $\mathrm{Cu}(\mathrm{I})$-catalyzed asymmetric allylation and propargylation of ketones. J. Am. Chem. Soc. 2010, 132, 6638-6639.

\subsection{Enantioselective allyl additions to carbonyl-containing compounds with non-metal- based catalysts}

a) Lou, S.; Moquist, P. N.; Schaus, S. E. Asymmetric allylboration of ketones catalyzed by chiral diols. J. Am. Chem. Soc. 2006, 128, 12660-12661. 
b) Barnett, D. S.; Moquist, P. N.; Schaus, S. E. The mechanism and an improved asymmetric allylboration of ketones catalyzed by chiral biphenols. Angew. Chem., Int. Ed. 2009, 48, 8679-8682.

c) Alam, R.; Vollgraff, T.; Eriksson, L.; Szabó, K. J. Synthesis of adjacent quaternary stereocenters by catalytic asymmetric allylboration. J. Am. Chem. Soc. 2015, 137, $11262-11265$.

d) Silverio, D. L.; Torker, S.; Pilyugina, T.; Vieira, E. M.; Snapper, M. L.; Haeffner, F.; Hoveyda, A. H. Simple organic molecules as catalysts for enantioselective synthesis of amines and alcohols. Nature 2013, 494, 216-221 (2013).

e) Lee, K.; Silverio, D. L.; Torker, S.; Robbins, D. W.; Haeffner, F.; Van Der Mei, F. W.; Hoveyda, A. H. Catalytic enantioselective addition of organoboron reagents to fluoroketones controlled by electrostatic interactions. Nat. Chem. 2016, 8, 768-777.

f) Robbins, D. W.; Lee, K. A.; Silverio, D. L.; Volkov, A.; Torker, S.; Hoveyda, A. H. Practical and broadly applicable catalytic enantioselective additions of allyl-B(pin) compounds to ketones and $\alpha$-ketoesters. Angew. Chem., Int. Ed. 2016, 55, 9610-9614.

\subsection{Enantioselective reductive coupling of ketones and allyl acetate with Ir-based catalysts}

a) Brito, G. A.; Jung, W. O.; Yoo, M.; Krische, M. J. Enantioselective iridium-catalyzed allylation of acetylenic ketones via 2-propanol-mediated reductive coupling of allyl acetate: C14-C23 of Pladienolide D. Angew. Chem., Int. Ed. 2019, 58, 18803-18807.

\subsection{Proline-catalyzed Mannich reactions with $\alpha$-hydroxy ketones}

a) List, B. Pojarliev P.; Biller, W. T.; Martin, H. J. The proline-catalyzed direct asymmetric three-component Mannich reaction: scope, optimization, and application to the highly enantioselective synthesis of 1,2-amino alcohols. J. Am. Chem. Soc. 2002, 124, 827-833.

\subsection{Enantioselective synthesis of $\alpha$-Hydroxy aryl ketones, cyclic ketones and $\beta$ - ketoesters}

a) Hayashi, Y.; Yamaguchi, J.; Sumiya, T.; Shoji, M. Direct proline-catalyzed asymmetric $\alpha$-aminoxylation of ketones. Angew. Chem. Int. Ed. 2004, 43, 1112-1115.

b) Yao, H.; Lian, M.; Li, Z.; Wang, Y.; Meng, Q. Asymmetric direct $\alpha$-hydroxylation of $\beta$-oxo esters catalyzed by chiral quaternary ammonium salts derived from cinchona alkaloids. J. Org. Chem. 2012, 77, 9601-9608.

c) Zou, L.; Wang, B.; Mu, H.; Zhang, H.; Song, Y.; Qu, J. Development of tartaric acid derive chiral guanidines and their application to catalytic enantioselective a-hydroxylation of $\beta$-dicarbonyl compounds. Org. Lett. 2013, 15, 3106-3109.

d) Wang, Y.; Yin, H.; Tang, X.; Wu, Y.; Meng, Q.; Gao, Z. A series of cinchona-derived $\mathrm{N}$-oxide phase-transfer catalysts: application to the photo-organocatalytic 
enantioselective $\alpha$-hydroxylation of $\beta$-dicarbonyl compounds. J. Org. Chem. 2016, 81, $7042-7050$.

e) Vila, C.; Quintero, L.; Blay, G.; Muñoz, M. C.; Pedro, J. R. Organocatalytic enantioselective synthesis of $\alpha$-hydroxyketones through a Friedel-Crafts reaction of naphthols and activated phenols with aryl- and alkylglyoxal hydrates. Org. Lett. 2016, $18,5652-5655$.

2.8 Synthesis of $\alpha$-hydroxy ketones and derivatives by catalytic enantioselective allylic substitution

a) Huo, X.; He, R.; Zhang, X.; Zhang, W. An Ir/Zn dual catalysis for enantio- and diastereodivergent $\alpha$-allylation of $\alpha$-hydroxyketones. J. Am. Chem. Soc. 2016, 138, 11093-11096.

2.9 Catalytic enantioselective dihydroxyl additions to enol ethers derived from ketones

a) Hashiyama, T.; Morikawa, K.; Sharpless, B. K. $\alpha$-Hydroxy ketones in high enantiomeric purity from asymmetric dihydroxylation of enol ethers. J. Org. Chem. 1992, 57, 5067-5068.

\section{Solvents and Reagents}

Acetonitrile was purchased from Fisher Scientific and distilled through $\mathrm{CaH}_{2}$ prior to use.

Acetic acid was purchased from Fisher Scientific and used as received.

Allylamine was purchased from Aldrich and purified by distillation from $\mathrm{CaH}_{2}$ (Strem) prior to use.

Allyl cyanide was purchased from Sigma-Aldrich and distilled through $\mathrm{CaH}_{2}$ prior to use (allyl cyanide was dried over $3 \AA$ molecule sieves prior to the distillation).

4-Aminobenzonitrile was purchased from Oakwood Chemical and purified by recrystallization and dried under vacuum prior to use.

6-Azidohexanenitrile was prepared according to previously reported procedures. ${ }^{1}$

Benzonitrile (anhydrous grade) was purchased from Sigma-Aldrich and used as received.

Bis(pinacolato)diboron $\left[\mathbf{B}_{2}(\mathbf{p i n})_{2}\right]$ was purchased from Frontier, recrystallized from hexanes and dried under vacuum prior to use.

(1,3-Bis-(2,4,6-trimethylphenyl)-2-imidazolidinylidene)dichloro(o-

isopropoxyphenylmethylene)ruthenium catalyst was purchased from AK Scientific and used as received.

3-Bromo-1-(trimethylsilyl)-1-propyne was purchased from Combi-Blocks and used as received.

Boron trifluoride diethyl etherate was purchased from Sigma-Aldrich and used as received.

1,2-Butadiene was purchased from SynQuest and used as a 4.6 M solution in THF, which was stored at $-40{ }^{\circ} \mathrm{C}$ (freezer). 
But-3-yn-1-yl (t-butoxycarbonyl)-L-valinate was purchased for SynQuest and used as received.

Cerium(III) chloride (anhydrous grade) was purchased from Strem Chemicals Inc. and used as received.

$\mathrm{CeCl}_{3} \bullet \mathbf{3 i}$-PrOH was prepared according to previously reported procedures. ${ }^{2,3}$

Cerium(III) triisopropoxide was prepared according to previously reported procedures. ${ }^{2,3}$

3-Chloroperbenzoic acid was purchased from Oakwood Chemical and used as received.

3-Chloropropionaldehyde diethylacetal was purchased from Sigma-Aldrich and used as received.

Chlorotriisopropoxytitanium(IV) was purchased from Sigma-Aldrich and used as received.

Cobalt(II) acetylacetonate: purchased from Alfa Aesar and used as received.

Copper(I) chloride was purchased from Strem and used as received.

Copper(I) cyanide was purchased from TCI and used as received.

$\mathbf{C u}\left(\mathbf{P P h}_{3}\right)_{3} \mathbf{F} \bullet 2 E t O H$ was prepared according to previously reported procedures. ${ }^{4}$

Cyclohexyl allene was purchased from Aldrich and used as received.

Dess-Martin periodinane (DMP) was purchased from Oakwood Chemical and used as received.

Dicyclohexylamine was purchased from Aldrich and purified by distillation from $\mathrm{CaH}_{2}$ (Strem) prior to use.

2-Dicyclohexylphosphino-2',6'-dimethoxybiphenyl (SPhos) was purchased from Aldrich and used as received.

Di-tert-butyl(methyl)phosphonium tetrafluoroborate: purchased from TCI and used as received

Ethynylbenzene was purchased from Aldrich and used as received.

Gilbert-Seyferth Reagent was prepared according to previously reported procedures. ${ }^{5}$

(E)-Hex-2-enenitrile was prepared according to a previously reported procedure. ${ }^{6}$

Imidazole was purchased from Oakwood Chemical and used as received.

Lithium bis(trimethylsilyl)amide solution 1.0 M in THF: purchased from Aldrich Chemical Co. and used as received

Lithium di-tert-butylbiphenylide (LiDBB) was prepared according to previously reported procedures. $^{7}$

2,6-Lutidine: purchased from Fluorochem and used as received.

Manganese(II) pivalate was prepared according to previously reported procedure. ${ }^{8}$

Mesitylcopper(I) was prepared according to previously reported procedures. ${ }^{9,10}$

Methanol was purchased from anhydrous from Fisher Scientific and used as received.

Methyl iodide: purchased from Alfa Aesar and used as received. 
Methylmagnesium chloride solution (3.0 $\mathrm{M}$ in THF) was purchased from Sigma-Aldrich and used as received.

Palladium(II) acetate was purchased from Strem and used as received.

Paraformaldehyde was purchased from Aldrich and used as received.

Pent-4-ynoic acid was purchased from Combi-Blocks and purified by recrystallization and dried under vacuum prior to use.

6-(4-Phenyl-1H-1,2,3-triazol-1-yl)hexanenitrile was prepared according to a reported procedure $^{11}$

(R)-2-Phenylpropanenitrile and $(\boldsymbol{S})$-2-phenylpropanenitrile was prepared according to previous reported procedures. ${ }^{12}$

3-Phenylprop-2-yn-1-ol was purchased from Aldrich and used as received.

Phenyllithium (1.9 $\mathrm{M}$ in $\left.(\boldsymbol{n}-\mathbf{B u})_{2} \mathrm{O}\right)$ was purchased from Aldrich and used as received.

Phenylsilane: purchased from Aldrich Chemical Co. and used as received.

Phosphines were purchased from Strem Chemicals Inc. and used as received.

Potassium carbonate was purchased from Aldrich Chemical Co. and used as received.

Potassium $t$-butoxide was purchased from Strem Chemical Inc. and used as received.

Potassium carbonate was purchased from Aldrich and used as received.

Potassium fluoride was purchased from Acros Organics and used as received.

Propan-2-ol was purchased from Fisher Scientific and purified by distillation from $\mathrm{CaH}_{2}$ (Strem) prior to use.

Propargyl alcohol was purchased from Aldrich and used as received.

Sodium borohydride was purchased from Oakwood Chemical and used as received.

Sodium iodide was purchased from Alfa Aesar and used as received.

Sodium methoxide was purchased from Strem and used as received.

Sodium $\boldsymbol{t}$-butoxide was purchased from Strem and used as received.

Sodium perborate tetrahydrate was purchased from Aldrich Chemical Co. and used as received.

tert-Amyl alcohol: purchased from TCI and purified by distillation over magnesium turnings (Fluorochem) prior to use.

$t$-Butyldimethylsilyl chloride was purchased from Oakwood Chemical and used as received. t-Butyllithium (1.6 $\mathrm{M}$ in pentane) was purchased from Aldrich and used as received.

t-Butyl-(Z)-4-(2-cyanovinyl)piperidine-1-carboxylate was synthesized according to a reported procedure. ${ }^{13}$

4-(4,4,5,5-Tetramethyl-1,3,2-dioxaborolan-2-yl)benzonitrile was purchased from Oakwood Chemical and purified by recrystallization and dried under vacuum prior to use. 
2-(Trifluoromethyl)benzonitrile was purchased from Oakwood Chemical and purified by distillation from $\mathrm{CaH}_{2}$ (Strem) prior to use.

Trimethylsilyl trifluoromethanesulfonate: purchased from TCI and used as received.

3-(Triisopropylsilyl)propiolonitrile was synthesized according to a reported procedure. ${ }^{14}$

Tetrabutylammonium fluoride solution (1.0 M in THF) was purchased from Sigma-Aldrich and used as received.

Vinylmagnesium bromide solution (1.0 M in THF) was purchased from Aldrich and used as received.

Zinc powder: purchased from Strem Chemicals Inc. and used as received.

\section{Preparation of Allenes}

Allenes were synthesized in several ways, based on previously reported procedures, as summarized below. ${ }^{15,16}$

\subsection{Through Crabbé processes}

\subsection{1 t-Butyldimethyl(penta-3,4-dien-1-yloxy)silane}

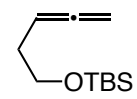

Spectroscopic data were consistent with those previously reported. ${ }^{17}$

\subsubsection{Methyl hexa-4,5-dienoate}

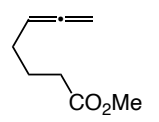

Light yellow oil, $5.11 \mathrm{~g}, 40.50 \mathrm{mmol}, 81 \%$ yield; IR (neat): 2953 (w), 1956 (w), 1736 (s), 1436 (m), 1159 (s), 845 (m) cm ${ }^{-1} ;{ }^{1} \mathbf{H}$ NMR (400 MHz, CDCl $\left.\mathbf{~}_{3}\right): \delta 5.16(\mathrm{p}, \mathrm{J}=6.5 \mathrm{~Hz}, 1 \mathrm{H})$, $4.71(\mathrm{dt}, \mathrm{J}=6.5,3.4 \mathrm{~Hz}, 2 \mathrm{H}), 3.67(\mathrm{~s}, 3 \mathrm{H}), 2.48-2.40(\mathrm{~m}, 2 \mathrm{H}), 2.36-2.25(\mathrm{~m}, 2 \mathrm{H}) .{ }^{13} \mathbf{C}$ NMR (101 MHz, CDCl $\mathbf{3}$ ): $\delta$ 208.5, 173.6, 88.9, 76.1, 51.7, 33.3, 23.3. HRMS (DART): Calcd for $\mathrm{C}_{7} \mathrm{H}_{10} \mathrm{O}_{2}[\mathrm{M}+\mathrm{H}]^{+}:$126.0681, Found: 126.0683 .

\subsection{3 t-Butyl hexa-4,5-dienoate}

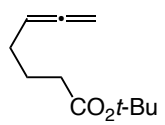

Spectroscopic data were consistent with those previously reported. ${ }^{18}$

\subsubsection{Methyl buta-2,3-dien-1-ylcarbamate}

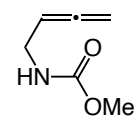

Spectroscopic data were consistent with those previously reported. ${ }^{19}$

\subsection{5 t-Butyl buta-2,3-dien-1-ylcarbamate}

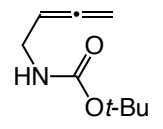


Spectroscopic data were consistent with those previously reported. ${ }^{20}$

\subsubsection{Penta-3,4-dien-1-yl ( $t$-butoxycarbonyl)-L-valinate}

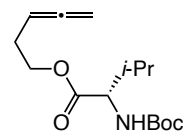

Pale-yellow oil; 1.46 g, 5.15 mmol, 58\% yield, >99:1 e.r.; IR (neat): 3364 (br), 2964 (m), 2931 (w), 2873 (w), 1710 (s), 1498 (m), 1390 (m), 1364 (m), 1308 (m), 1244 (w), 1156 (s), 1088 (m), 1015 (m), 845 (m), 779 (w) cm ${ }^{-1} ;{ }^{1} \mathbf{H}$ NMR (500 MHz, CDCl $): \delta 5.04$ (td, $J=14.2,7.3$ $\mathrm{Hz}, 1 \mathrm{H}), 5.01$ (br, 1H), 4.86-4.60 (m, 2H), 4.30-3.93 (m, 3H), 2.39-2.24 (m, 2H), 2.17-2.02 $(\mathrm{m}, 1 \mathrm{H}), 1.41(\mathrm{~s}, 9 \mathrm{H}), 0.93(\mathrm{~d}, J=6.9 \mathrm{~Hz}, 3 \mathrm{H}), 0.86(\mathrm{~d}, J=6.9 \mathrm{~Hz}, 3 \mathrm{H}$; the two sets of signals for the major diastereomer are due to carbamate rotamers; $\left.{ }^{13} \mathbf{C ~ N M R ~ ( 1 2 6 ~} \mathbf{~ M H z}, \mathbf{C D C l}_{3}\right): \delta$ 209.1, 172.4, 155.7, 128.4, 85.8, 79.7, 75.6, 64.1, 58.6, 31.4, 28.4, 27.8, 19.0, 17.6; HRMS (DART): Calcd for $\mathrm{C}_{15} \mathrm{H}_{26} \mathrm{NO}_{4} \mathrm{~S}[\mathrm{M}+\mathrm{H}]^{+}:$284.1856, Found: 284.1850; specific rotation: $[\alpha]_{\mathrm{D}}{ }^{20}=+0.2\left(c 1.0, \mathrm{CHCl}_{3}\right)$ for an enantiomerically enriched sample of $>99: 1$ e.r.

Enantiomeric purity was determined by HPLC analysis in comparison with authentic racemic material; Chiralcel OZ-H column, 98:2 hexanes/i-PrOH, $0.5 \mathrm{~mL} / \mathrm{min}, 220 \mathrm{~nm}$.
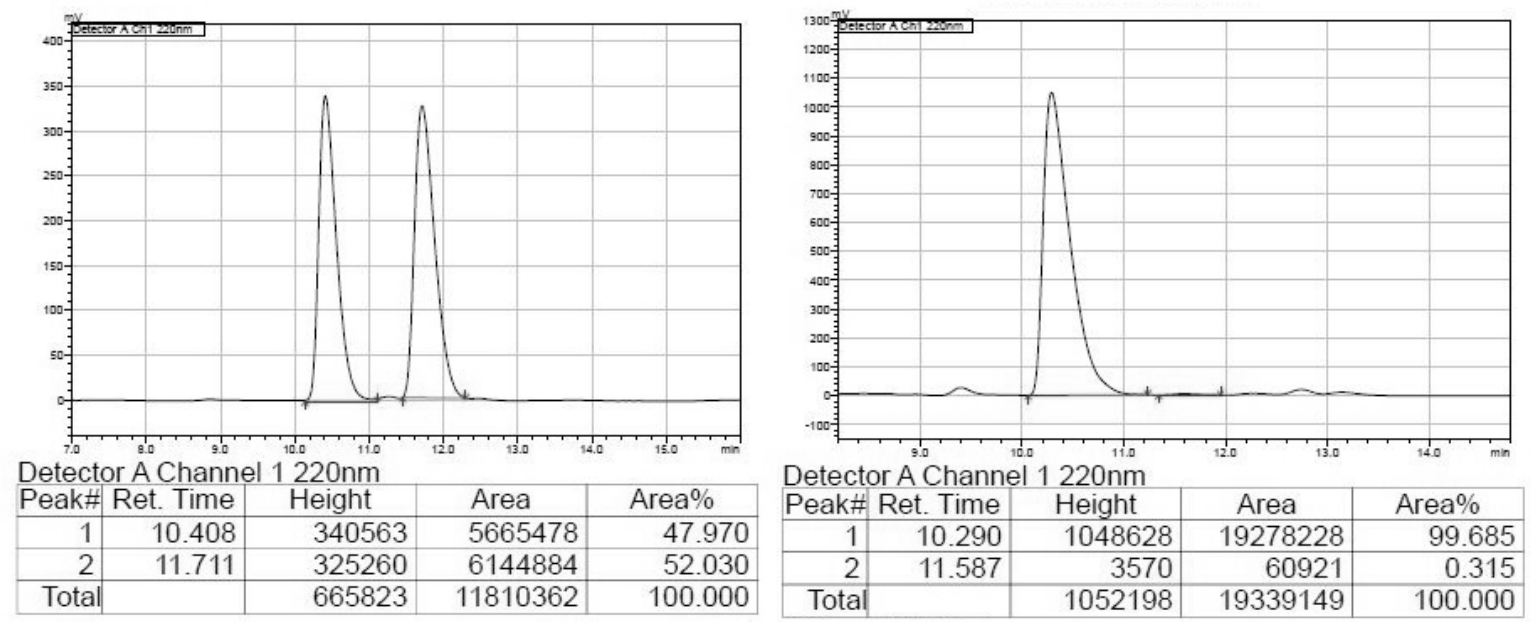

\begin{tabular}{|c|c|c|c|c|c|}
\hline Retention Time & Area & Area $\%$ & Retention Time & Area & Area\% \\
\hline 10.408 & 5665478 & 47.970 & 10.290 & 19278228 & 99.685 \\
\hline 11.711 & 6144884 & 52.030 & 11.587 & 60921 & 0.315 \\
\hline
\end{tabular}

\subsubsection{Propa-1,2-dien-1-ylbenzene}

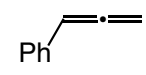

Spectroscopic data were consistent with those previously reported. ${ }^{21}$

\subsection{Through allylic substitutions}
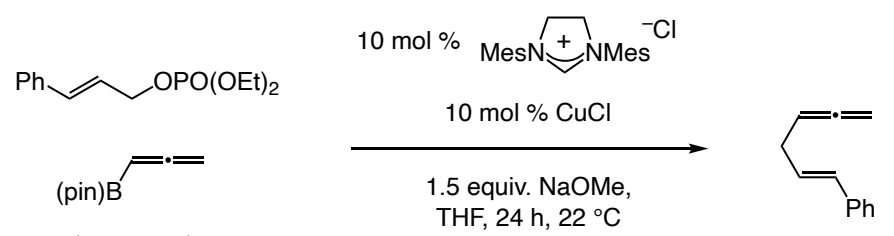


\subsection{1 (E)-Hexa-1,4,5-trien-1-ylbenzene}

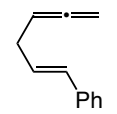

Spectroscopic data were consistent data reported previously. ${ }^{22}$

\subsection{Through propargylic substitutions}

A previously reported protocol was followed. ${ }^{23}$

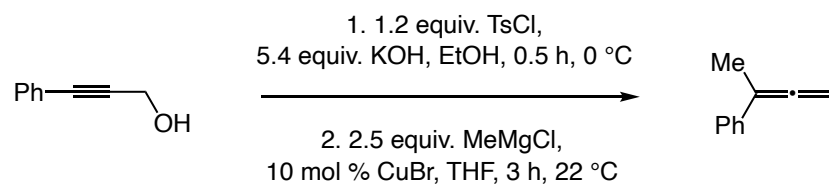

\subsubsection{Buta-2,3-dien-2-ylbenzene}

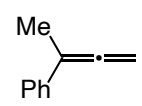

Spectroscopic data were consistent with those reported previously. ${ }^{24}$
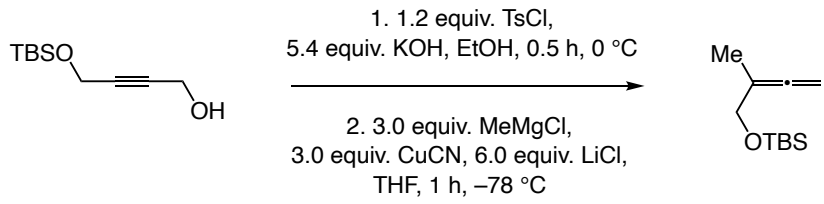

\subsection{2 $t$-Butyldimethyl((2-methylbuta-2,3-dien-1-yl)oxy)silane}

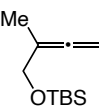

Spectroscopic data were consistent with those reported previously. ${ }^{25}$

\subsection{Preparation of $\boldsymbol{O}$-substituted allenes}

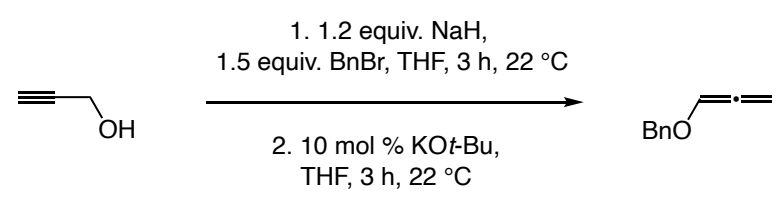

\subsection{1 ((Propa-1,2-dien-1-yloxy)methyl)benzene}

$$
\mathrm{BnO}^{\mathrm{P}}=
$$

Spectroscopic data were consistent with those reported previously. ${ }^{26}$

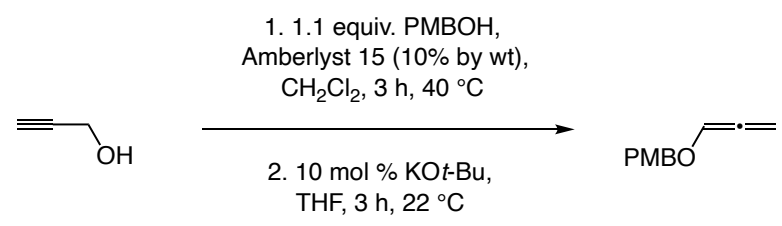

\subsubsection{1-Methoxy-4-((propa-1,2-dien-1-yloxy)methyl)benzene}

$$
\mathrm{PMBO}^{=}=
$$

Spectroscopic data were consistent with those reported previously. ${ }^{26}$ 


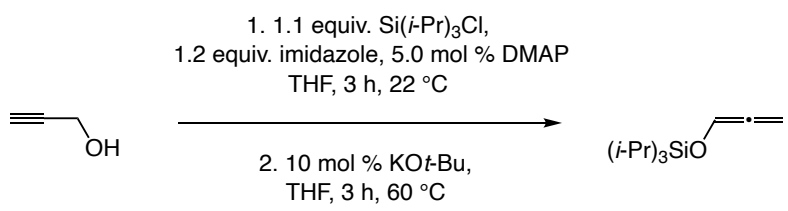

\subsection{3 t-Butyldimethyl((2-methylbuta-2,3-dien-1-yl)oxy)silane}

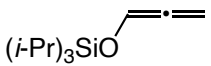

Spectroscopic data were consistent with those reported previously. ${ }^{27}$

\section{Preparation of Nitriles}

\subsubsection{4-(Trimethylsilyl)but-3-ynenitrile}<smiles>NCC#C[AsH3]</smiles>

A two-necked $50 \mathrm{~mL}$ flask equipped with a stir bar was charged with $\mathrm{CuCN}(1.97 \mathrm{~g}, 22.0$ $\mathrm{mmol}$ ), after which equipped with a reflux condenser connected to $\mathrm{N}_{2}$ inlet at $22{ }^{\circ} \mathrm{C}$. The flask was sealed with a septum, and 3-bromo-1-(trimethylsilyl)-1-propyne (3.02 g, $15.8 \mathrm{mmol}$ ) was added by syringe. The mixture was allowed to heat to $100{ }^{\circ} \mathrm{C}$ (rigorous vigorous), at which time the mixture turned black. A large volume of a dark foam was generated within $30 \mathrm{~min}$. (CAUTION: The reaction must be carried out behind a shield in a fume hood and with significant head space in the flask because of the considerable increase in vapor pressure.) The mixture was kept for $16 \mathrm{~h}$ at $100{ }^{\circ} \mathrm{C}$, after which the black slurry was washed with $\mathrm{Et}_{2} \mathrm{O}(3 \mathrm{x}$ $50 \mathrm{~mL}$ ). After filtration, the combined organic layers were dried over $\mathrm{MgSO}_{4}$, filtered again and volatiles were removed in vacuo, affording a residue, which was purified by silica gel chromatography $\left(\mathrm{R}_{f}=0.15,4: 1\right.$ hexanes: $\left.\mathrm{Et}_{2} \mathrm{O}\right)$ affording the desired product as yellow oil (195 $\mathrm{mg}, 1.42 \mathrm{mmol}, 10 \%$ yield) in a containing $5 \%$ of the allenyl isomer and traces of unidentified $\left({ }^{1} \mathrm{H}\right.$ NMR analysis). Yellow oil; IR (neat): 2957 (w), 2926 (w), 2188 (w), 1913 (w), 1250 (m), 843 (s), 761 (w) cm ${ }^{-1} ;{ }^{1} \mathbf{H}$ NMR (500 MHz, $\mathbf{C D C l}_{3}$ ): $\delta 3.37$ (s, 2H), 0.17 (s, 9H); ${ }^{\mathbf{1 3}} \mathbf{C}$ NMR (126 MHz, $\mathbf{C D C l}_{3}$ ): $\delta 114.3,91.6,89.8,10.4,-0.3$. HRMS (DART): Calcd for $\mathrm{C}_{7} \mathrm{H}_{12} \mathrm{NSi}$ $[\mathrm{M}+\mathrm{H}]^{+}: 138.0734$ Found: 138.0739 .

\section{Synthesis of $\beta, \gamma$-unsaturated ketones}

\subsection{Reactions with monosubstituted allenes}

In a $\mathrm{N}_{2}$-filled glovebox, an oven-dried vial $(4 \mathrm{~mL}, 17 \times 38 \mathrm{~mm})$ was charged with $\mathrm{MesCu}(\mathrm{I})$ (0.9 mg, $0.0050 \mathrm{mmol})$, phos-1 $(3.3 \mathrm{mg}, 0.006 \mathrm{mmol})$ and THF $(0.50 \mathrm{~mL})$. The vial was sealed (screw cap) and the solution was agitated ( $\sim 1 \mathrm{~min})$ until the solution became homogeneous. The mixture was kept at $22{ }^{\circ} \mathrm{C}$ for $10 \mathrm{~min}$ after which $\mathrm{MeOH}$ (4.8 mg, $0.15 \mathrm{mmol}, 1.5$ equiv.) was added by syringe. The mixture was kept at $22^{\circ} \mathrm{C}$ for another $10 \mathrm{~min}$ before being allowed to cool to $-40{ }^{\circ} \mathrm{C}$ (placed in a freezer) and remain at this temperature for $1 \mathrm{~h}$. A separate ovendried vial was charged with PhCN (10.3 mg, $0.10 \mathrm{mmol}$ ), $t$-butyldimethyl(penta-3,4-dien-1yloxy)silane (23.8 mg, $0.12 \mathrm{mmol}), \mathrm{B}_{2}(\text { pin })_{2}(30.5 \mathrm{mg}, 0.12 \mathrm{mmol})$ and THF $(0.50 \mathrm{~mL})$. The vial was capped and the solution was agitated manually (swirling) until the solution became 
homogeneous ( $\sim 1 \mathrm{~min})$. The mixture was then allowed to cool to $-40{ }^{\circ} \mathrm{C}$ for $1 \mathrm{~h}$ (freezer). The latter mixture was then charged with the aforementioned solution of the $\mathrm{Cu}$ complex through pre-cooled syringe (kept in glovebox freezer at $-40{ }^{\circ} \mathrm{C}, 1 \mathrm{~h}$ ) in a single portion. The vial was re-sealed (screw cap) and the mixture was allowed to remain at $-40{ }^{\circ} \mathrm{C}$ for $16 \mathrm{~h}$, after which the vial was removed from the glove box without allowing the solution to warm, and the reaction was quenched by the addition of a saturated aqueous solution of $\mathrm{NH}_{4} \mathrm{Cl}(1 \mathrm{~mL})$ followed by vigorous shaking of the mixture. The solution was washed with $\mathrm{Et}_{2} \mathrm{O}(3 \times 10 \mathrm{~mL})$ and the combined organic layers were dried over $\mathrm{MgSO}_{4}$. Filtration and removal of the volatiles in vacuo afforded orange oil, which was purified by silica gel chromatography $(20: 1 \rightarrow 10: 1$ hexanes: $\left.\mathrm{Et}_{2} \mathrm{O}\right)$ to furnish ketone $\mathbf{2 k}$ as colorless oil $(38.7 \mathrm{mg}, 0.090 \mathrm{mmol}, 90 \%$ yield). The product must be stored at $-20{ }^{\circ} \mathrm{C}$ to prevent decomposition.

NOTE: For most cases, $\mathrm{CuF}\left(\mathrm{PPh}_{3}\right)_{3} \cdot 2 \mathrm{EtOH}(4.8 \mathrm{mg}, 0.0050 \mathrm{mmol}, 5.0 \mathrm{~mol} \%)$ can be used instead of $\mathrm{Mes} \mathrm{Cu}$. The reactions may be run in a fume hood (vessels were connected to a $\mathrm{N}_{2}-$ filled Schlenk line) without appreciable change in yield or e.r. The mixtures were kept at $22^{\circ} \mathrm{C}$ for 20-30 min, reaction progress was monitored by ${ }^{1} \mathrm{H}$ and ${ }^{11} \mathrm{~B}$ NMR spectroscopy and/or TLC until full conversion. In certain cases, it was preferable to perform a reaction with CuMes at $40{ }^{\circ} \mathrm{C}$. Such instances were as follows: (i) when the reaction generated a ketone that contained a particularly sensitive stereogenic center (e.g., 2i), (ii) when the nitrile contained a strongly electron-deficient moiety (e.g., $\mathbf{2 a}, \mathbf{4 j - k}$ ), or (iii) when the NH-ketimine could intramolecularly displace a leaving group (4a).

\subsection{Reactions with aryl and heteroaryl nitriles}

\subsection{1 (R)-Methyl 5-(4,4,5,5-tetramethyl-1,3,2-dioxaborolan-2-yl)-4-(2-(trifluoromethyl) benzoyl)hex-5-enoate (2a)}

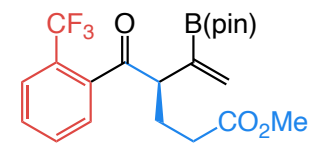

Pale-yellow oil; $32.0 \mathrm{mg}, 0.0751 \mathrm{mmol}, 75 \%$ yield, 98.5:1.5 e.r.; IR (neat): 2978 (w), 1736 (m), 1701 (m), 1313 (s), 1136 (s), 857 (w), 769 (m) cm ${ }^{-1} ;{ }^{1} \mathbf{H}$ NMR (500 MHz, CDCl $\mathbf{~}_{3}$ ): $\delta 7.67$ $(\mathrm{dd}, J=7.4,1.7 \mathrm{~Hz}, 1 \mathrm{H}), 7.57-7.45(\mathrm{~m}, 3 \mathrm{H}), 6.03(\mathrm{~d}, J=2.4 \mathrm{~Hz}, 1 \mathrm{H}), 5.76(\mathrm{~d}, J=2.4 \mathrm{~Hz}, 1 \mathrm{H})$, $3.98(\mathrm{dd}, J=8.6,5.9 \mathrm{~Hz}, 1 \mathrm{H}), 3.65(\mathrm{~s}, 3 \mathrm{H}), 2.34(\mathrm{dd}, J=8.7,7.1 \mathrm{~Hz}, 2 \mathrm{H}), 2.31-2.23(\mathrm{~m}, 1 \mathrm{H})$, 2.10-2.01 (m, 1H), 1.19 (s, 6H) $1.16(\mathrm{~s}, 6 \mathrm{H}) ;{ }^{13} \mathbf{C}$ NMR (151 MHz, CDCl 3 ): $\delta 203.5,173.8$, 139.8, 137.9 (C-B bs), 133.9 (q, $J=4.7 \mathrm{~Hz}), 131.5,130.0,128.7,127.6$ (q, $J=32.1 \mathrm{~Hz}), 126.8$ $(\mathrm{q}, J=5.2 \mathrm{~Hz}), 123.7$ (q, $J=273.8 \mathrm{~Hz}), 83.9,55.2,51.6(\mathrm{q}, J=3.8 \mathrm{~Hz}), 31.8,25.9,24.8,24.7$; ${ }^{11}$ B NMR (128 MHz, $\left.\mathbf{C D C l}_{3}\right) \delta 29.0 ;{ }^{19} \mathbf{F}$ NMR (376 $\left.\mathbf{M H z}, \mathbf{C D C l}_{3}\right): \delta-57.8$ (s); HRMS (DART): Calcd for $\mathrm{C}_{21} \mathrm{H}_{27} \mathrm{BF}_{3} \mathrm{O}_{5}[\mathrm{M}+\mathrm{H}]^{+}: 427.1898$, Found: 427.1904; specific rotation: $[\alpha]_{\mathrm{D}^{20}}=-24.6\left(c 1.0, \mathrm{CHCl}_{3}\right)$ for an enantiomerically enriched sample of 98.5:1.5 e.r.

Enantiomeric purity was determined by HPLC analysis in comparison with authentic racemic material; Chiralcel OZ-H column, 98:2 hexanes/i-PrOH, $0.8 \mathrm{~mL} / \mathrm{min}, 220 \mathrm{~nm}$. 

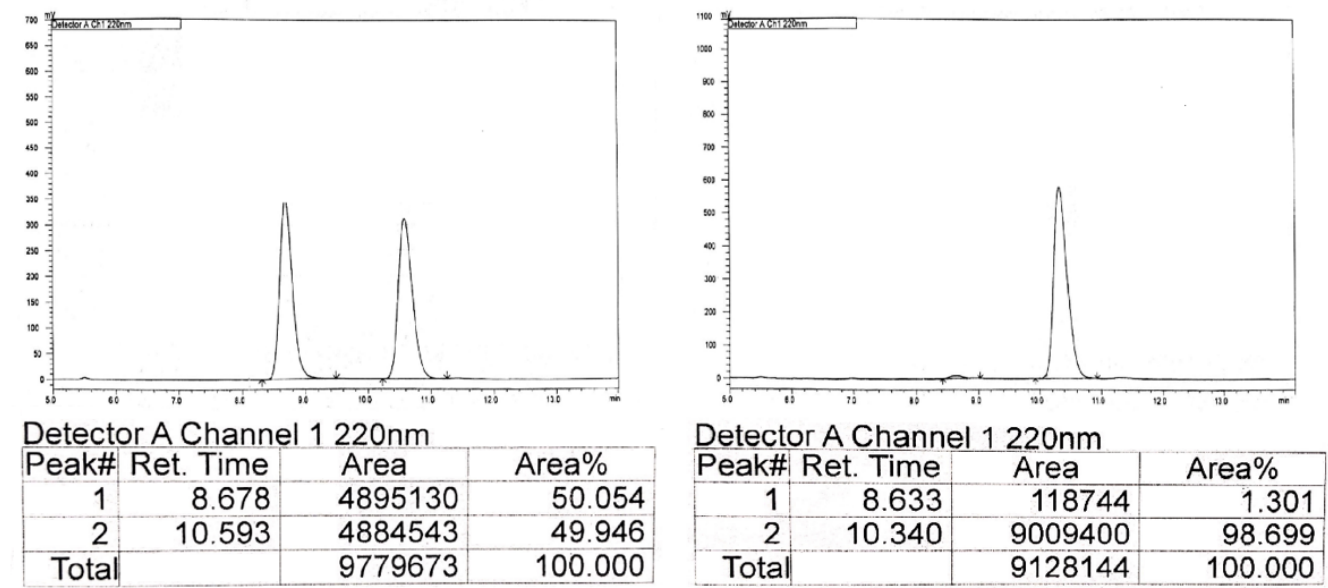

\begin{tabular}{|c|c|c|c|c|c|}
\hline Retention Time & Area & Area $\%$ & Retention Time & Area & Area\% \\
\hline 8.678 & 4895130 & 50.054 & 8.633 & 118744 & 1.301 \\
\hline 10.593 & 4884543 & 49.946 & 10.340 & 9009400 & 98.699 \\
\hline
\end{tabular}

6.2.2 Methyl $(S)-(3-(4,4,5,5$-tetramethyl-1,3,2-dioxaborolan-2-yl)-2-(4-(4,4,5,5-tetramethyl-1,3,2-dioxaborolan-2-yl)benzoyl)but-3-en-1-yl)carbamate (2b)<smiles>C=C(Br)C(CNC(C)=O)C(=O)c1ccc(Br)cc1</smiles>

Pale-yellow oil; 34.5 mg, 0.0711 mmol, 81\% yield, 99:1 e.r.; IR (neat): 3364 (br), 2975 (m), 2928 (w), 1724 (m), 1507 (m), 1432 (m), 1357 (s), 1137 (s), 1088 (s), 961 (m), 853 (m), 562 (m) $\mathrm{cm}^{-1}$; ${ }^{1} \mathbf{H}$ NMR (400 MHz, $\left.\mathbf{C D C l}_{3}\right): \delta 7.96-7.80(\mathrm{~m}, 4 \mathrm{H}), 5.96(\mathrm{~s}, 1 \mathrm{H}), 5.62(\mathrm{~s}, 1 \mathrm{H}), 5.30$ $4.90(\mathrm{~m}, 1 \mathrm{H}), 4.47$ (t, $J=6.5 \mathrm{~Hz}, 1 \mathrm{H}), 3.74-3.43(\mathrm{~m}, 5 \mathrm{H}), 1.34(\mathrm{~s}, 12 \mathrm{H}), 1.29(\mathrm{~s}, 6 \mathrm{H}), 1.27$ (s, $6 \mathrm{H})$. The two sets of signals are due to equilibrium of carbamate rotamers; ${ }^{13} \mathbf{C}$ NMR (151 MHz, $\left.\mathbf{C D C l}_{3}\right): \delta$ 200.5, 157.1, 138.1, 137.3 (C-B bs), 134.9, 133.8, 128.0, 84.3, 52.1 51.4, 42.8, 29.9, 25.0, 24.9; ${ }^{11} \mathbf{B}$ NMR (128 $\left.\mathbf{M H z}, \mathbf{C D C l}_{3}\right): \delta 30.1$; HRMS (DART): Calcd for $\mathrm{C}_{25} \mathrm{H}_{38} \mathrm{BNO}_{7}[\mathrm{M}+\mathrm{H}]^{+}: 486.2829$, Found: 486.2803 ; specific rotation: $[\alpha]_{\mathrm{D}}{ }^{20}=-4.7$ (c 1.0, $\mathrm{CHCl}_{3}$ ) for an enantiomerically enriched sample of 99:1 e.r.

Enantiomeric purity was determined by HPLC analysis in comparison with authentic racemic material; Chiralcel OZ-H column, 90:10 hexanes/i-PrOH, $0.8 \mathrm{~mL} / \mathrm{min}, 254 \mathrm{~nm}$.
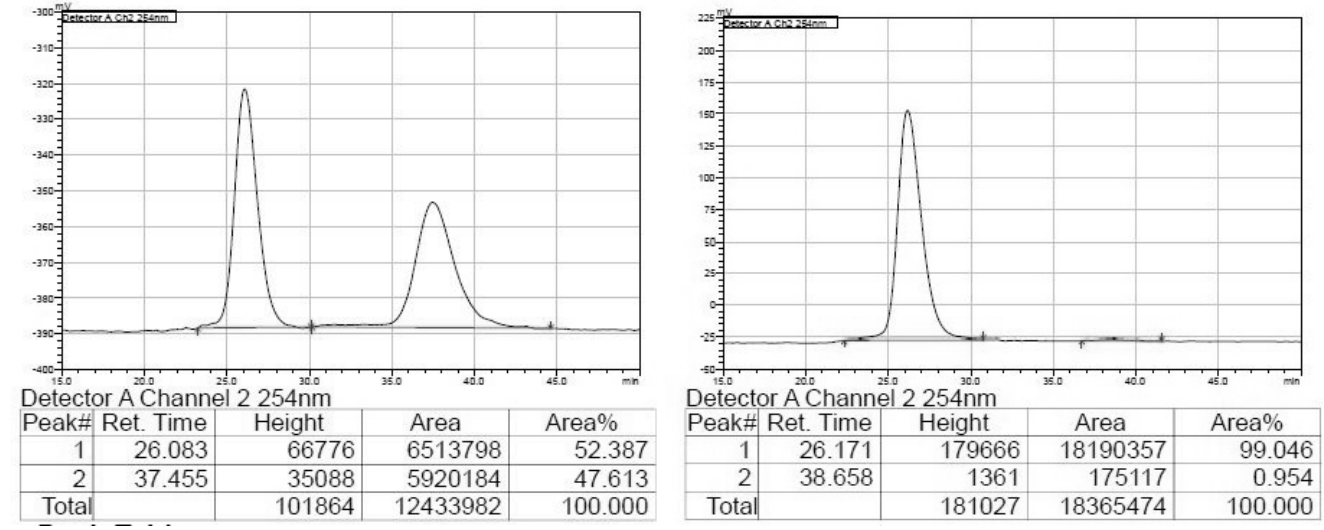

\begin{tabular}{|c|c|c|c|c|c|}
\hline Retention Time & Area & Area $\%$ & Retention Time & Area & Area $\%$ \\
\hline
\end{tabular}




\begin{tabular}{|l|l|l|l|l|l|}
\hline 26.083 & 6513798 & 52.387 & 26.171 & 18190357 & 99.046 \\
\hline 37.455 & 5920184 & 47.613 & 38.658 & 175117 & 0.954 \\
\hline
\end{tabular}

6.2.3 (R)-1-(4-Bromophenyl)-2-methyl-3-(4,4,5,5-tetramethyl-1,3,2-dioxaborolan-2yl)but-3-en-1-one (2c)

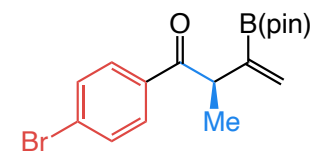

Modified procedure: $2.0 \mathrm{~mol} \%$ CuMes (18.2 mg, $0.100 \mathrm{mmol})$ and $2.2 \mathrm{~mol} \%$ phos-1 (59.6 $\mathrm{mg}, 0.110 \mathrm{mmol}$ ) were used.

White solid, m.p. $=46-48{ }^{\circ} \mathrm{C} ; 1.62 \mathrm{~g}, 4.43 \mathrm{mmol}, 89 \%$ yield, 98.5:1.5 e.r.; IR (neat): 2975 (m), 2928 (w), 1683 (s), 1583 (m), 1353 (m), 1312 (s), 1136 (s), 965 (m), 854 (m) cm ${ }^{-1}$; ${ }^{1} \mathbf{H}$ NMR (CDCl 3 , 500 MHz): $\delta 7.83(\mathrm{~d}, J=8.5 \mathrm{~Hz}, 2 \mathrm{H}), 7.54$ (d, $J=8.5 \mathrm{~Hz}, 2 \mathrm{H}), 5.91$ (d, $J=2.3$ $\mathrm{Hz}, 1 \mathrm{H}), 5.63(\mathrm{~d}, J=2.3 \mathrm{~Hz}, 1 \mathrm{H}), 4.24(\mathrm{q}, J=6.8 \mathrm{~Hz}, 1 \mathrm{H}), 1.30$ (d, $J=6.8 \mathrm{~Hz}, 3 \mathrm{H}), 1.26$ (s, 12H); ${ }^{13} \mathbf{C ~ N M R ~ ( C D C l} 3,101$ MHz): $\delta$ 200.6, 141.2 (C-B, bs), 135.5, 131.7, 131.3, 130.4, 127.7, 84.1, 45.1, 25.0, 24.8, 17.5; ${ }^{11} \mathbf{B}$ NMR (CDCl 3 , 160 MHz): $\delta 30.0$; HRMS (DART): Calcd for $\mathrm{C}_{17} \mathrm{H}_{23} \mathrm{BO}_{3} \mathrm{Br}[\mathrm{M}+\mathrm{H}]^{+}: 365.0918$, Found: 365.0924 ; specific rotation: $[\alpha]_{\mathrm{D}}{ }^{20}=-58.4$ (c $0.54, \mathrm{CHCl}_{3}$ ) for an enantiomerically enriched sample of $98.5: 1.5$ e.r.

Enantiomeric purity was determined by HPLC analysis in comparison with authentic racemic material; $(R, R)$-Whelk-O1 column, 99.9:0.1 hexanes $/ i-\mathrm{PrOH}, 1.0 \mathrm{~mL} / \mathrm{min}, 254 \mathrm{~nm}$.
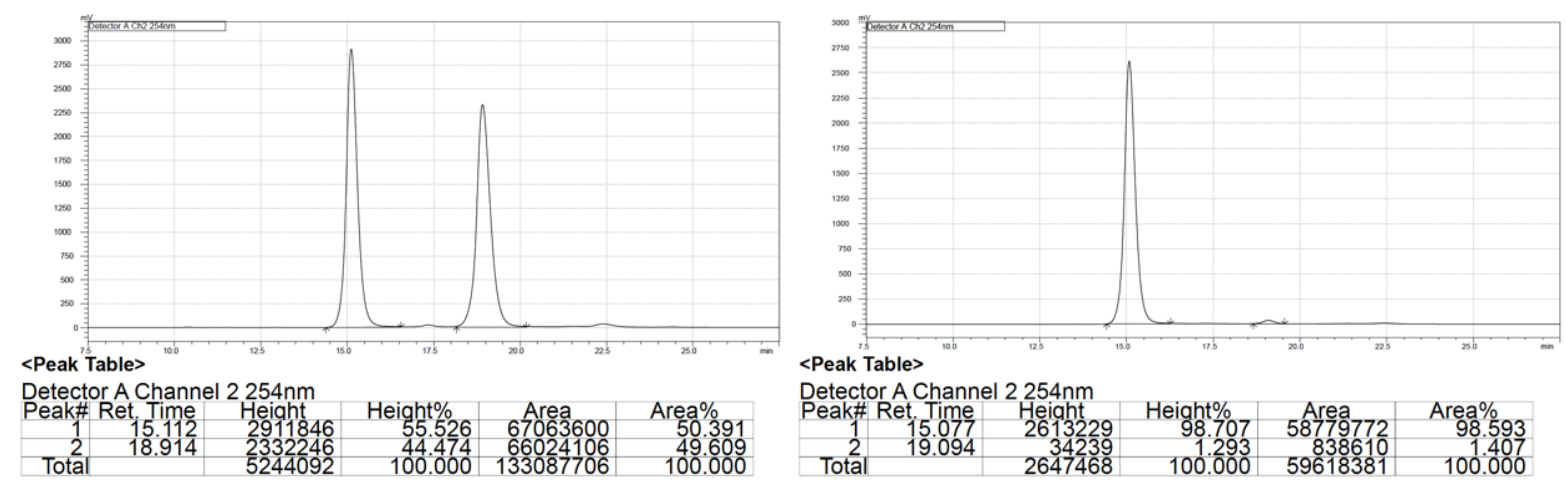

\begin{tabular}{|c|c|c|c|c|c|}
\hline Retention Time & Area & Area\% & Retention Time & Area & Area\% \\
\hline 15.112 & 67063600 & 50.391 & 15.077 & 58779772 & 98.593 \\
\hline 18.914 & 66024106 & 49.609 & 19.094 & 838610 & 1.407 \\
\hline
\end{tabular}

6.2.4 t-Butyl $(R)$-4-(4-aminobenzoyl)-5-(4,4,5,5-tetramethyl-1,3,2-dioxaborolan-2-yl) hex-5-enoate (2d)

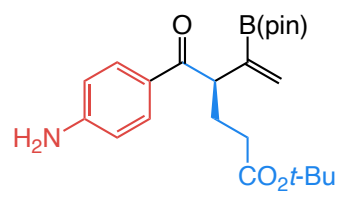

Yellow oil; 36.5 mg, 0.0879 mmol, 88\% yield, >99:1 e.r.; IR (neat): 3462 (w), 3365 (m), 3244 (w), 3054 (s), $2974(\mathrm{~m}), 1721$ (m), 1628 (m), 1594 (s), 1367 (m), 13101 (m), 1144 (s), $965(\mathrm{w})$, $847(\mathrm{w}) \mathrm{cm}^{-1} ;{ }^{1} \mathbf{H}$ NMR (600 MHz, $\left.\mathbf{C D C l}_{3}\right): \delta 7.87-7.81(\mathrm{~m}, 2 \mathrm{H}), 6.62-6.55(\mathrm{~m}, 2 \mathrm{H}), 5.90(\mathrm{~d}$, 
$J=2.5 \mathrm{~Hz}, 1 \mathrm{H}), 5.71-5.67(\mathrm{~m}, 1 \mathrm{H}), 4.19(\mathrm{t}, J=7.0 \mathrm{~Hz}, 1 \mathrm{H}), 4.08(\mathrm{~s}, 2 \mathrm{H}), 2.31-2.22(\mathrm{~m}, 1 \mathrm{H})$, 2.22-2.15 (m, 2H), 1.96-1.89 (m, 1H), $1.43(\mathrm{~s}, 9 \mathrm{H}), 1.27(\mathrm{~s}, 12 \mathrm{H}) ;{ }^{13} \mathrm{C}$ NMR (151 MHz, $\left.\mathbf{C D C l}_{3}\right): \delta 198.4,173.0,151.0,140.1$ (C-B bs), 131.7, 131.2, 127.3, 113.8, 83.9, 80.1, 49.0, 33.9, 28.2, 28.1, 25.0, 24.8; ${ }^{11}$ B NMR (192 MHz, $\left.\mathbf{C D C l}_{3}\right)$ : $\delta$ 29.3; HRMS (DART): Calcd for $\mathrm{C}_{23} \mathrm{H}_{35} \mathrm{BNO}_{5}[\mathrm{M}+\mathrm{H}]^{+}:$416.2603, Found: 416.2601; specific rotation: $[\alpha]_{\mathrm{D}}{ }^{20}=-32.1$ (c 1.0, $\mathrm{CHCl}_{3}$ ) for an enantiomerically enriched sample of $>99: 1$ e.r.

Enantiomeric purity was determined by HPLC analysis in comparison with authentic racemic material; Chiralcel OZ-H column, 90:10 hexanes/i-PrOH, $0.8 \mathrm{~mL} / \mathrm{min}, 210 \mathrm{~nm}$.
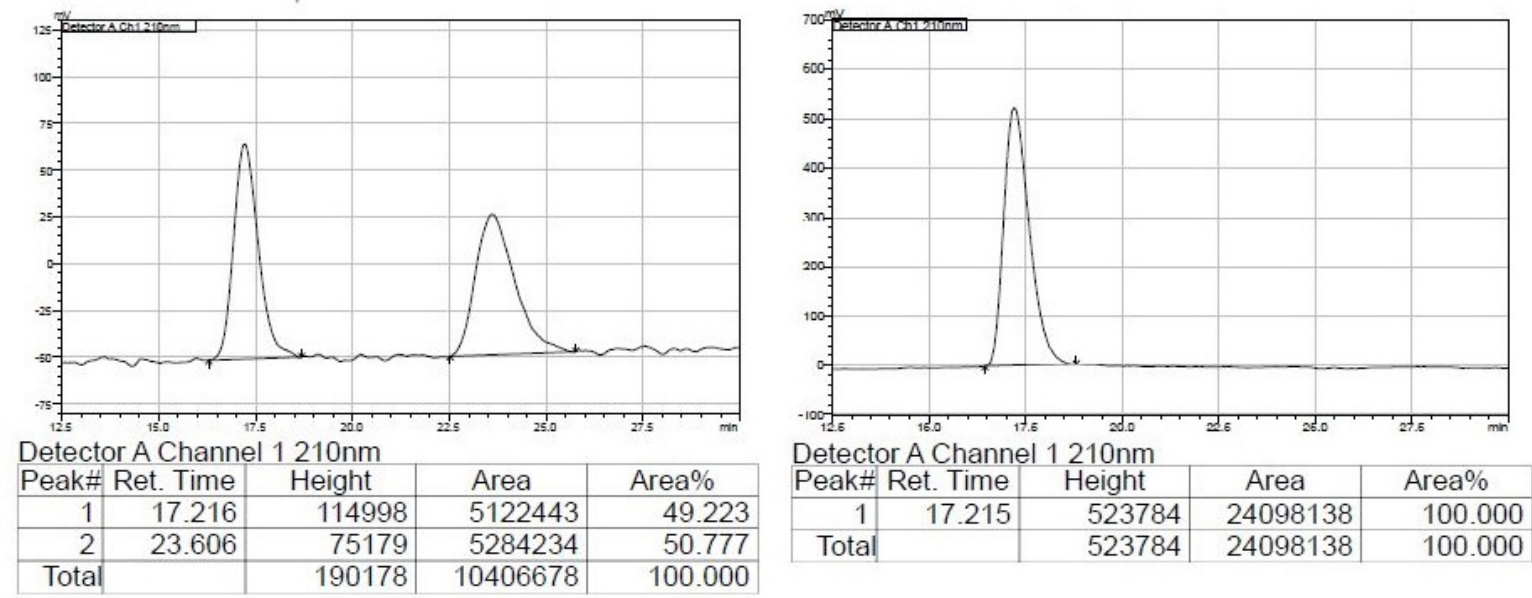

\begin{tabular}{|c|c|c|c|c|c|}
\hline Retention Time & Area & Area\% & Retention Time & Area & Area\% \\
\hline 17.716 & 5122443 & 49.223 & 17.215 & 24098138 & 100.000 \\
\hline 23.606 & 5284234 & 50.777 & & & \\
\hline
\end{tabular}

\subsection{5 (R)-1-(Benzofuran-2-yl)-2-(2-((t-butyldimethylsilyl)oxy)ethyl)-3-(4,4,5,5-} tetramethyl-1,3,2-dioxaborolan-2-yl)but-3-en-1-one (2e)

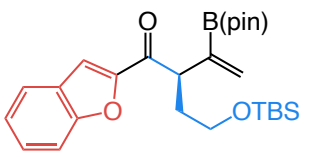

Colorless oil; $46.6 \mathrm{mg}, 0.099$ mmol, 99\% yield, 96:4 e.r.; IR (neat): 2925 (m), 2854 (w), 1679 (s), 1621 (m), 1554 (m), $832(\mathrm{~s}) \mathrm{cm}^{-1} ;{ }^{1} \mathbf{H}$ NMR (600 MHz, CDCl $): \delta 7.67$ (d, $J=7.8 \mathrm{~Hz}$, $1 \mathrm{H}), 7.63(\mathrm{~s}, 1 \mathrm{H}), 7.55(\mathrm{~d}, J=8.4 \mathrm{~Hz}, 1 \mathrm{H}), 7.49-7.42(\mathrm{~m}, 1 \mathrm{H}), 7.31-7.24(\mathrm{~m}, 1 \mathrm{H}), 5.96(\mathrm{~d}, J=$ $2.5 \mathrm{~Hz}, 1 \mathrm{H}), 5.78(\mathrm{~d}, J=2.5 \mathrm{~Hz}, 1 \mathrm{H}), 4.37$ (t, $J=7.0 \mathrm{~Hz}, 1 \mathrm{H}), 3.71-3.58(\mathrm{~m}, 2 \mathrm{H}), 2.30-2.22$ (m, 1H), 1.96-1.89 (m, 1H), 1.29 (s, 6H), 1.27 (s, 6H), 0.89 (s, 9H), $0.02(\mathrm{~s}, 3 \mathrm{H}), 0.00(\mathrm{~s}, 3 \mathrm{H})$; ${ }^{13}$ C NMR (151 MHz, $\left.\mathbf{C D C l}_{3}\right): \delta 191.4,155.8,152.6,139.4$ (C-B bs), 131.9, 128.1, 127.3, $123.8,123.2,113.9,112.6,83.95,61.0,48.3,35.0,29.8,26.1,24.9,18.4,-5.2,-5.3 ;{ }^{11}$ B NMR (192 MHz, CDCl $\mathbf{~}_{3}$ ): $\delta$ 29.4; HRMS (DART): Calcd for $\mathrm{C}_{26} \mathrm{H}_{40} \mathrm{BO}_{5} \mathrm{Si}[\mathrm{M}+\mathrm{H}]^{+}: 471.2721$, Found: 471.2745; specific rotation: $[\alpha]_{\mathrm{D}}{ }^{20}=-36.5\left(c\right.$ 1.0, $\left.\mathrm{CHCl}_{3}\right)$ for an enantiomerically enriched sample of 96:4 e.r.

Enantiomeric purity was determined by HPLC analysis in comparison with authentic racemic material; $(R, R)$-Whelk-O1 column, 99.5:0.5 hexanes/i-PrOH, $0.5 \mathrm{~mL} / \mathrm{min}, 220 \mathrm{~nm}$. 

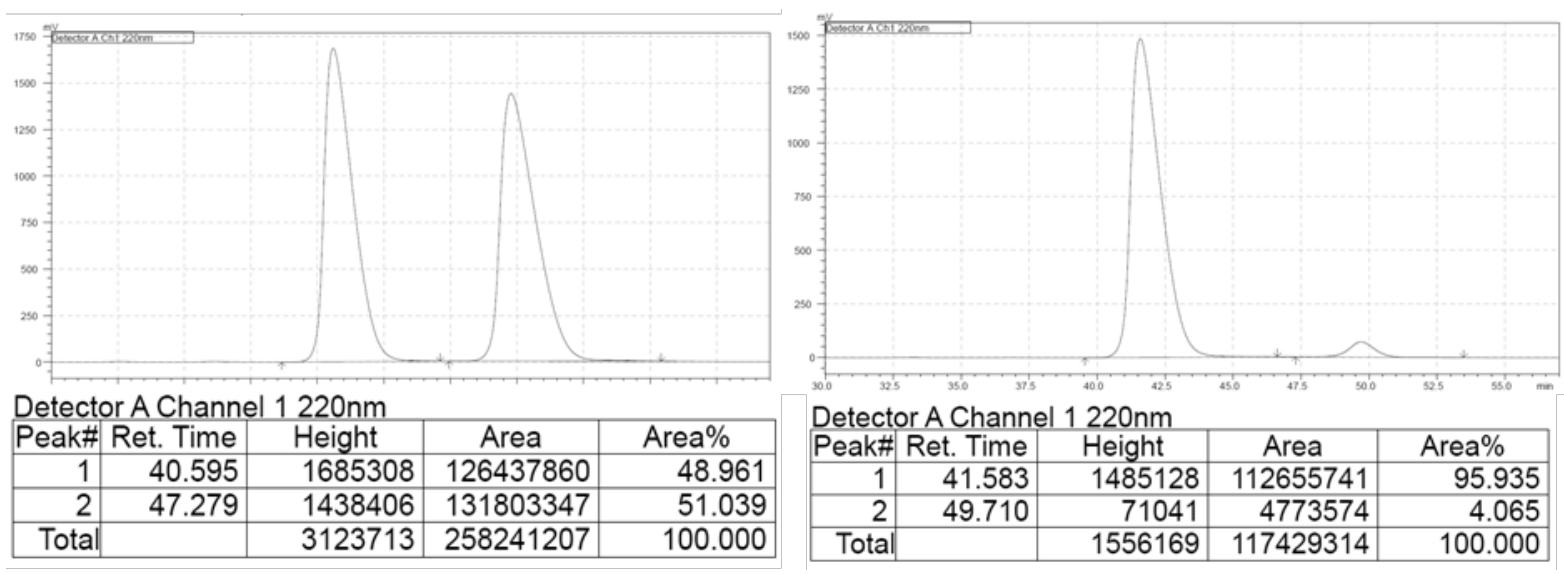

\begin{tabular}{|c|c|c|c|c|c|}
\hline Retention Time & Area & Area $\%$ & Retention Time & Area & Area\% \\
\hline 40.595 & 126437860 & 48.961 & 41.583 & 112655741 & 95.935 \\
\hline 47.279 & 131803347 & 51.039 & 49.710 & 4773574 & 4.065 \\
\hline
\end{tabular}

6.2.6 t-Butyl-(R)-5-(2-(2-((t-butyldimethylsilyl)oxy)ethyl)-3-(4,4,5,5-tetramethyl-1,3,2dioxaborolan-2-yl)but-3-enoyl)-1H-indole-1-carboxylate (2f)

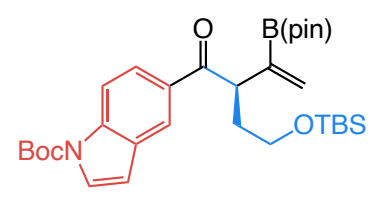

Colorless oil; 53.5 mg, 0.094 mmol, 95\% yield, 98:2 e.r.; IR (neat): 2926 (m), 2854 (w), 1737 (s), 1673 (s), 1607 (m), 1534 (m), 947 (s), 832 (s) cm ${ }^{-1}$; ${ }^{1}$ H NMR (600 MHz, CDCl 3 ): $\delta 8.29$ $(\mathrm{d}, J=1.7 \mathrm{~Hz}, 1 \mathrm{H}), 8.13(\mathrm{~d}, J=8.7 \mathrm{~Hz}, 1 \mathrm{H}), 8.01(\mathrm{dd}, J=8.7,1.8 \mathrm{~Hz}, 1 \mathrm{H}), 7.62(\mathrm{~d}, J=3.8$ $\mathrm{Hz}, 1 \mathrm{H}), 6.61(\mathrm{~d}, J=3.8, \mathrm{~Hz}, 1 \mathrm{H}), 5.89(\mathrm{~d}, J=2.5 \mathrm{~Hz}, 1 \mathrm{H}), 5.69(\mathrm{~d}, J=2.5 \mathrm{~Hz}, 1 \mathrm{H}), 4.56$ (t, $J$ $=6.9 \mathrm{~Hz}, 1 \mathrm{H}), 3.73-3.55(\mathrm{~m}, 2 \mathrm{H}), 2.27-2.19(\mathrm{~m}, 1 \mathrm{H}), 1.95-1.88(\mathrm{~m}, 1 \mathrm{H}), 1.67(\mathrm{~s}, 9 \mathrm{H}), 1.29(\mathrm{~s}$, 6H), 1.28 (s, 6H), 0.90 (s, 9H), 0.03 (s, 3H), -0.01 (s, 3H); $\left.{ }^{13} \mathbf{C ~ N M R ~ ( 1 5 1 ~ M H z , ~ C D C l ~}\right): \delta$ 200.5, 149.6, 137.8, 131.9, 131.3, 130.4, 127.1, 125.2, 122.7, 114.9, 108.1, 84.4, 83.9, 61.2, $47.3,35.7,28.3,26.2,24.9,18.4,-5.1,-5.2$. The carbon bearing the boron atom could not be detected due to quadrupolar effects; ${ }^{11} \mathbf{B}$ NMR (192 $\left.\mathbf{M H z}, \mathbf{C D C l}_{3}\right)$ : $\delta$ 29.4; HRMS (DART): Calcd for $\mathrm{C}_{31} \mathrm{H}_{49} \mathrm{~B}_{\mathrm{N}} \mathrm{O}_{6} \mathrm{Si}[\mathrm{M}+\mathrm{H}]^{+}: 570.3417$, Found: 570.3417 ; specific rotation: $[\alpha]_{\mathrm{D}}{ }^{20}=-$ 60.2 (c 1.0, $\mathrm{CHCl}_{3}$ ) for an enantiomerically enriched sample of 98:2 e.r.

Enantiomeric purity was determined by HPLC analysis in comparison with authentic racemic material; $(R, R)-$ Whelk-O1 column, 99.5:0.5 hexanes/i-PrOH, $0.5 \mathrm{~mL} / \mathrm{m}$ in, $220 \mathrm{~nm}$. 

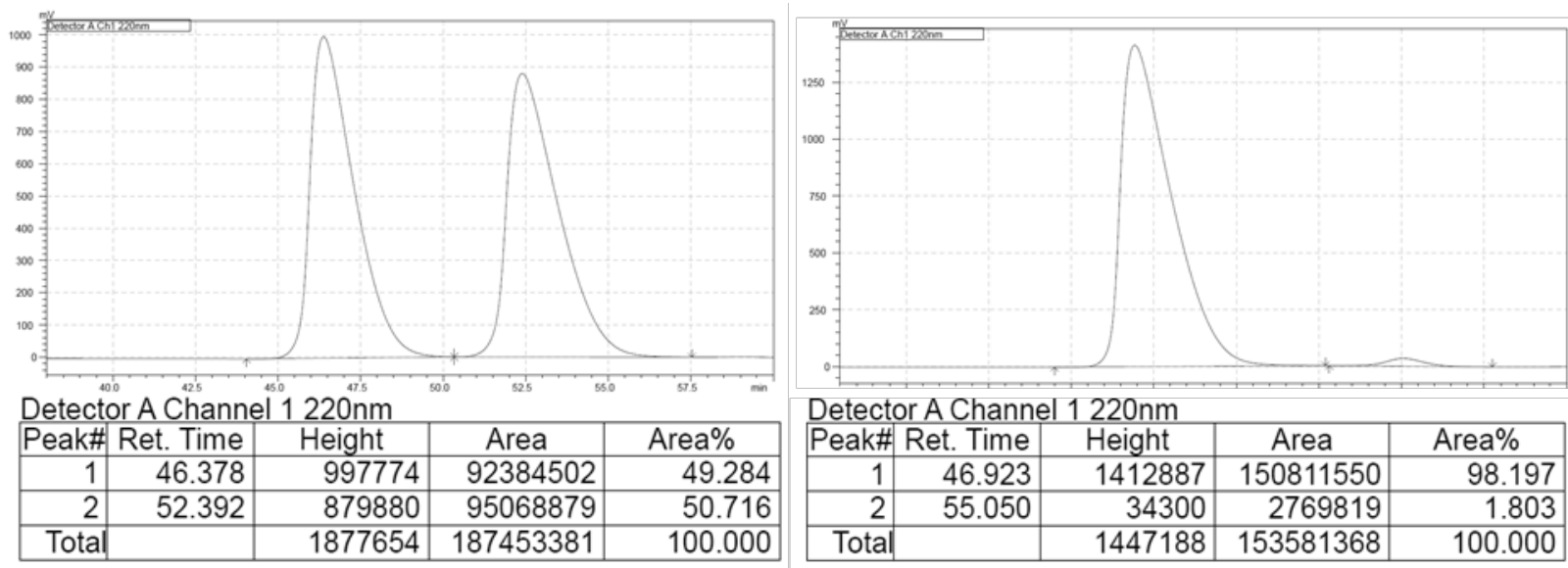

\begin{tabular}{|c|c|c|c|c|c|}
\hline Retention Time & Area & Area\% & Retention Time & Area & Area $\%$ \\
\hline 46.378 & 92384502 & 49.284 & 46.923 & 150811550 & 98.197 \\
\hline 52.392 & 95068879 & 50.716 & 55.050 & 2769819 & 1.803 \\
\hline
\end{tabular}

6.2.7 (R)-2-(2-((t-Butyldimethylsilyl)oxy)ethyl)-1-(pyridin-3-yl)-3-(4,4,5,5-tetramethyl1,3,2-dioxaborolan-2-yl)but-3-en-1-one (2g)<smiles>C=C(Br)C(CC[SeH])C(=O)c1cccnc1</smiles>

Colorless oil; $36.2 \mathrm{mg}, 0.084 \mathrm{mmol}, 91 \%$ yield, 97.5:2.5 e.r.; IR (neat): 2954 (m), 2926 (m), 2738 (m), 2856 (w), 1686 (m), 1342 (s), 835 (s) cm ${ }^{-1} ;{ }^{1}$ H NMR (400 MHz, CDCl 3 ): $\delta 9.21$ (bs, 1H), 8.73 (bs, 1H), 8.25 (d, $J=8.0 \mathrm{~Hz}, 1 \mathrm{H}), 7.35$ (bs, 1H), 5.95 (d, $J=2.4 \mathrm{~Hz}, 1 \mathrm{H}), 5.68$ $(\mathrm{d}, J=2.4 \mathrm{~Hz}, 1 \mathrm{H}), 4.45(\mathrm{~m}, 1 \mathrm{H}), 3.62(\mathrm{~m}, 2 \mathrm{H}), 2.20(\mathrm{~m}, 1 \mathrm{H}), 1.85(\mathrm{~m}, 1 \mathrm{H}), 1.24(\mathrm{~s}, 12 \mathrm{H}), 0.86$ $(\mathrm{s}, 9 \mathrm{H}), 0.00(\mathrm{~s}, 3 \mathrm{H}),-0.03$ (s, 3H); ${ }^{13} \mathbf{C}$ NMR (101 MHz, $\left.\mathbf{C D C l}_{3}\right): \delta 199.5,152.9,150.3,139.3$ (C-B bs), 136.0, 132.2, 123.3, 83.9, 60.7, 47.5, 34.0, 29.7, 25.9, 24.92, 24.85, 18.2, -5.2, -5.3; ${ }^{11} \mathbf{B}$ NMR (160 MHz, CDCl 3 ): $\delta$ 29.5; HRMS (DART): Calcd for $\mathrm{C}_{23} \mathrm{H}_{39} \mathrm{BNO}_{4} \mathrm{Si}[\mathrm{M}+\mathrm{H}]^{+}$: 432.2741, Found: 432.2761; specific rotation: $\left.[\alpha]_{\mathrm{D}}{ }^{20}=-24.8(c) 1.0, \mathrm{CHCl}_{3}\right)$ for an enantiomerically enriched sample of 97.5:2.5 e.r.

Enantiomeric purity was determined by HPLC analysis in comparison with authentic racemic material; Chiralcel AD-H column, 100:0 hexanes/i-PrOH, $0.3 \mathrm{~mL} / \mathrm{min}, 254 \mathrm{~nm}$.
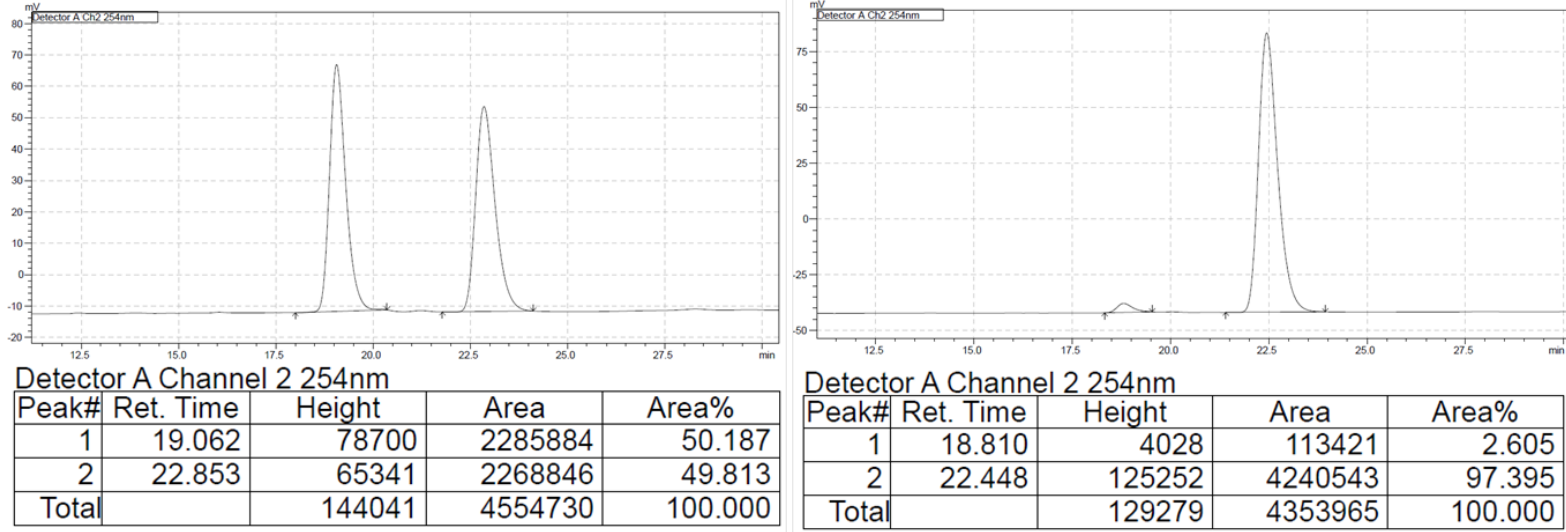

Retention Time

Area

Area $\%$

Retention Time

Area

Area\% 


\begin{tabular}{|l|l|l|l|l|l|}
\hline 19.062 & 2285884 & 50.187 & 18.810 & 113421 & 2.605 \\
\hline 22.853 & 2268846 & 49.813 & 22.448 & 4240543 & 97.395 \\
\hline
\end{tabular}

6.2.8 (S)-2-(2-((t-Butyldimethylsilyl)oxy)ethyl)-3-(4,4,5,5-tetramethyl-1,3,2dioxaborolan-2-yl)-1-(thiophen-2-yl)but-3-en-1-one (2h)

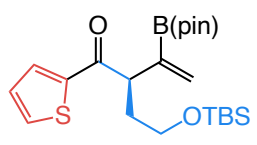

Colorless oil; 41.9 mg, 0.096 mmol, 96\% yield, 97.5:2.5 e.r.; IR (neat): 2952 (w), 2925 (w), 2853 (w), 1661 (s), 1611 (s), 1516 (s), 833 (s), 774 (w), 678 (s) cm ${ }^{-1} ;{ }^{1}$ H NMR (600 MHz, CDCl$\left._{3}\right): \delta 7.83(\mathrm{~d}, J=3.7 \mathrm{~Hz}, 1 \mathrm{H}), 7.57(\mathrm{~d}, J=4.9 \mathrm{~Hz}, 1 \mathrm{H}), 7.09-7.05(\mathrm{~m}, 1 \mathrm{H}), 5.94(\mathrm{~d}, J=$ $1.6 \mathrm{~Hz}, 1 \mathrm{H}), 5.76(\mathrm{~d}, J=1.6 \mathrm{~Hz}, 1 \mathrm{H}), 4.33(\mathrm{t}, J=7.0 \mathrm{~Hz}, 1 \mathrm{H}), 3.70-3.51(\mathrm{~m}, 2 \mathrm{H}), 2.24-2.16$ $(\mathrm{m}, 1 \mathrm{H}), 1.92-1.84(\mathrm{~m}, 1 \mathrm{H}), 1.26(\mathrm{~s}, 6 \mathrm{H}), 1.26(\mathrm{~s}, 6 \mathrm{H}), 0.88(\mathrm{~s}, 9 \mathrm{H}),-0.01(\mathrm{~s}, 3 \mathrm{H})-0.02(\mathrm{~s}$, $3 \mathrm{H}) ;{ }^{13} \mathbf{C}$ NMR (151 MHz, $\left.\mathbf{C D C l}_{3}\right): \delta$ 193.8, 144.8, 133.4, 132.5, 131.6, 127.9, 83.9, 61.0, $48.7,35.5,26.1,24.9,18.4,-5.2,-5.3$. The carbon bearing the boron atom could not be detected due to quadrupolar effects; ${ }^{11} \mathbf{B}$ NMR (192 $\left.\mathbf{M H z}, \mathbf{C D C l}_{3}\right)$ : $\delta$ 29.8; HRMS (DART): Calcd for $\mathrm{C}_{22} \mathrm{H}_{38} \mathrm{BO}_{4} \mathrm{SiS}[\mathrm{M}+\mathrm{H}]^{+}: 437.235$, Found: 437.2348; specific rotation: $[\alpha]_{\mathrm{D}}{ }^{20}=-18.7$ (c 0.20 $\mathrm{M}, \mathrm{CHCl}_{3}$ ) for an enantiomerically enriched sample of 97.5:2.5 e.r.

Enantiomeric purity was determined by HPLC analysis in comparison with authentic racemic material; Chiralcel OD-H column, 99.5:0.5 hexanes $/ i-\mathrm{PrOH}, 0.5 \mathrm{~mL} / \mathrm{min}, 220 \mathrm{~nm}$.
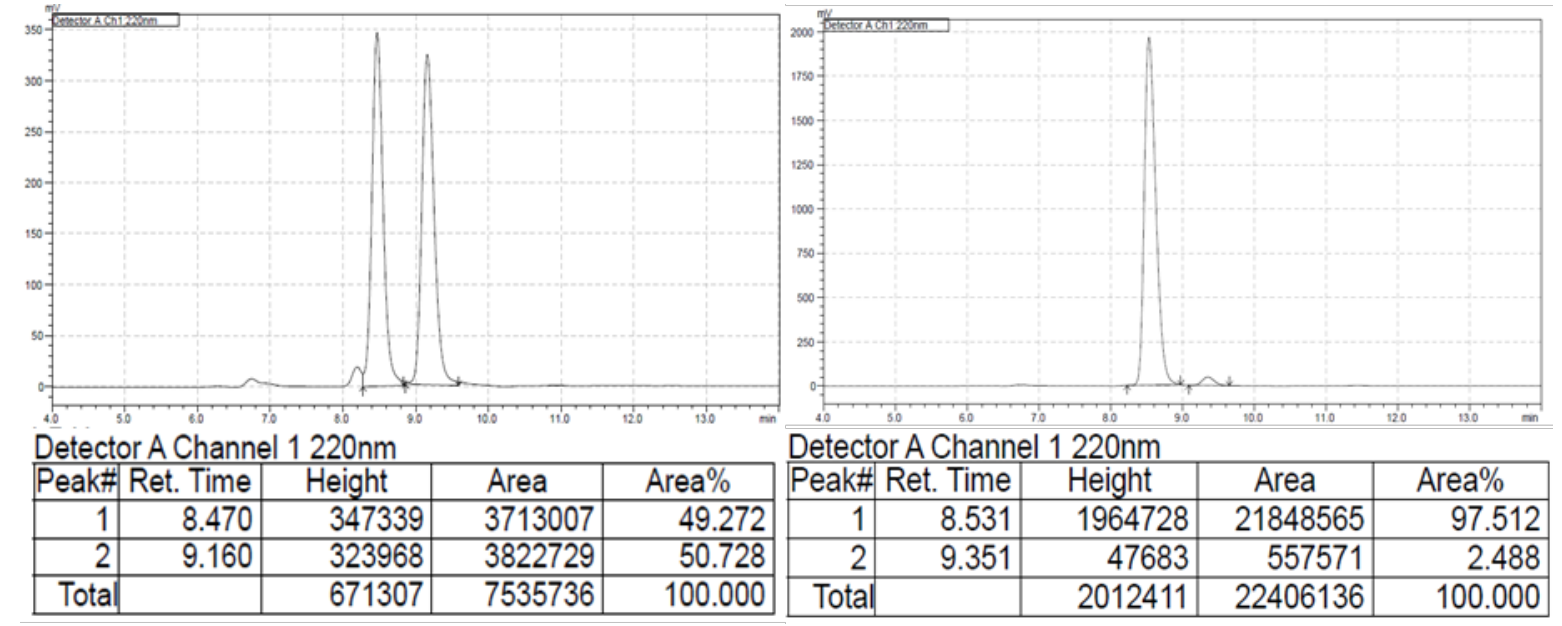

\begin{tabular}{|c|c|c|c|c|c|}
\hline Retention Time & Area & Area\% & Retention Time & Area & Area\% \\
\hline 8.470 & 3713007 & 49.272 & 8.531 & 21848565 & 97.512 \\
\hline 9.160 & 7535736 & 50.728 & 9.351 & 557571 & 2.488 \\
\hline
\end{tabular}

6.2.9 (S)-1,2-Diphenyl-3-(4,4,5,5-tetramethyl-1,3,2-dioxaborolan-2-yl)but-3-en-1-one (2i)<smiles>C=C(Br)C(=O)c1ccccc1</smiles>

Modified procedure: the reaction was quenched by addition of an aqueous solution of $\mathrm{HCl}(0.5$ M) at $-40{ }^{\circ} \mathrm{C}$ after which the mixture was shaken vigorously for one min. 
Colorless solid; m.p. $=103-105{ }^{\circ} \mathrm{C} ; 24.7 \mathrm{mg}, 0.071 \mathrm{mmol}, 71 \%$ yield, $95.5: 4.5$ e.r.; IR (neat): 3058 (w), 2974 (m), 2920 (m), 2848 (w), 1679 (s), 1596 (w), 1359 (s), 1311 (s), 698 (s) cm cm $^{-1}$ ${ }^{1}$ H NMR (400 MHz, CDCl 3$): \delta 7.98-7.93(\mathrm{~m}, 2 \mathrm{H}), 7.53-7.44(\mathrm{~m}, 1 \mathrm{H}), 7.42-7.34(\mathrm{~m}, 2 \mathrm{H})$, 7.34-7.28 (m, 2H), 7.26-7.20 (m, 3H), $6.01(\mathrm{~s}, 1 \mathrm{H}), 5.55(\mathrm{~s}, 1 \mathrm{H}), 5.26(\mathrm{~s}, 1 \mathrm{H}), 1.22(\mathrm{~s}, 6 \mathrm{H})$, 1.21 (s, 6H); ${ }^{13} \mathbf{C}$ NMR (151 MHz, $\left.\mathbf{C D C l}_{3}\right): \delta 199.4,141.3$ (C-B bs), 137.6, 137.1, 132.6, 131.0, 129.8, 129.1, 128.8, 128.5, 127.1, 83.9, 77.4, 77.2, 77.0, 58.4, 29.9, 25.0, 24.9, 24.64, 24.59, 24.5; ${ }^{11} \mathbf{B}$ NMR (128 MHz, $\mathbf{C D C l}_{3}$ ): $\delta$ 30.4, HRMS (DART): Calcd for $\mathrm{C}_{22} \mathrm{H}_{26} \mathrm{BO}_{3}$ $[\mathrm{M}+\mathrm{H}]^{+}:$349.1970, Found: 349.1963 ; specific rotation: $[\alpha]_{\mathrm{D}}{ }^{20}=+52.4\left(c 1.0, \mathrm{CHCl}_{3}\right)$ for an enantiomerically enriched sample of 95.5:4.5 e.r.

Enantiomeric purity was determined by HPLC analysis in comparison with authentic racemic material; Chiralcel OD-H column, 99.9:0.1 hexanes/i-PrOH, $0.5 \mathrm{~mL} / \mathrm{min}, 254 \mathrm{~nm}$
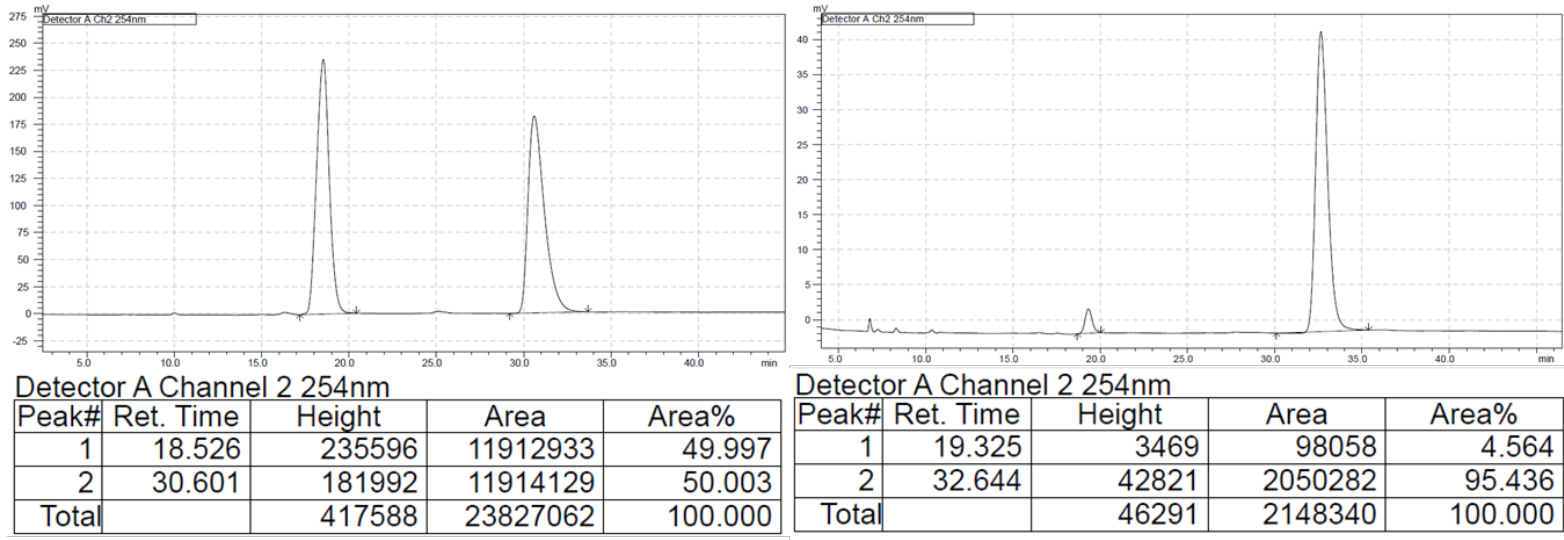

\begin{tabular}{|c|c|c|c|c|c|}
\hline Retention Time & Area & Area\% & Retention Time & Area & Area\% \\
\hline 18.596 & 11912933 & 49.997 & 19.325 & 98058 & 4.564 \\
\hline 13.027 & 11914129 & 50.003 & 32.644 & 2050282 & 95.436 \\
\hline
\end{tabular}

6.2.10 (R)-4-(2-Methyl-3-(4,4,5,5-tetramethyl-1,3,2-dioxaborolan-2-yl)but-3enoyl)benzenesulfonyl fluoride $(2 \mathrm{j})$

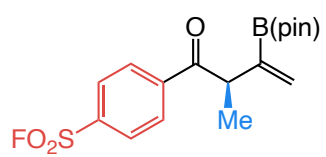

White solid, m.p. $=48-50{ }^{\circ} \mathrm{C} ; 30.2 \mathrm{mg}, 0.082 \mathrm{mmol}, 82 \%$ yield, $90: 10$ e.r.; IR (neat): 2976 (w), 2922 (m), 1693 (m), 1414 (m), 1213 (s), 967 (w), 776 (w) cm ${ }^{-1}$; ${ }^{1}$ H NMR (400 MHz, CDCl $\left._{3}\right): \delta 8.16(\mathrm{~d}, J=8.4 \mathrm{~Hz}, 2 \mathrm{H}), 8.05(\mathrm{~d}, J=8.4 \mathrm{~Hz}, 2 \mathrm{H}), 5.95(\mathrm{~d}, J=2.1 \mathrm{~Hz}, 1 \mathrm{H}), 5.64(\mathrm{bs}$, $1 \mathrm{H}), 4.28(\mathrm{q}, J=6.8 \mathrm{~Hz}, 1 \mathrm{H}), 1.33(\mathrm{~d}, J=6.8 \mathrm{~Hz}, 3 \mathrm{H}), 1.25(\mathrm{~s}, 12 \mathrm{H}) ;{ }^{13} \mathbf{C}$ NMR (101 MHz, $\left.\mathbf{C D C l}_{3}\right): \delta 199.9,142.5,136.0(\mathrm{~d}, J=25.4 \mathrm{~Hz}), 132.2,129.8,128.7,84.3,45.8,25.0,24.8$, 17.3. The carbon bearing the boron atom could not be detected due to quadrupolar effects; ${ }^{19} \mathbf{F}$

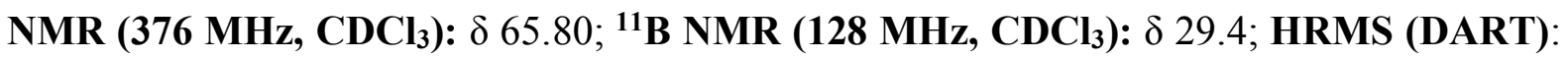
Calcd for $\mathrm{C}_{17} \mathrm{H}_{23} \mathrm{BO}_{5} \mathrm{FS}[\mathrm{M}+\mathrm{H}]^{+}: 369.1331$, Found: 369.1338 ; specific rotation: $[\alpha]_{\mathrm{D}}{ }^{20}=$ $-42.8\left(c\right.$ 1.0, $\left.\mathrm{CHCl}_{3}\right)$ for an enantiomerically enriched sample of 90:10 e.r.

Enantiomeric purity was determined by HPLC analysis in comparison with authentic racemic material; Chiralcel AD-H column, 100:0 hexanes/i-PrOH, $0.5 \mathrm{~mL} / \mathrm{min}, 220 \mathrm{~nm}$. 

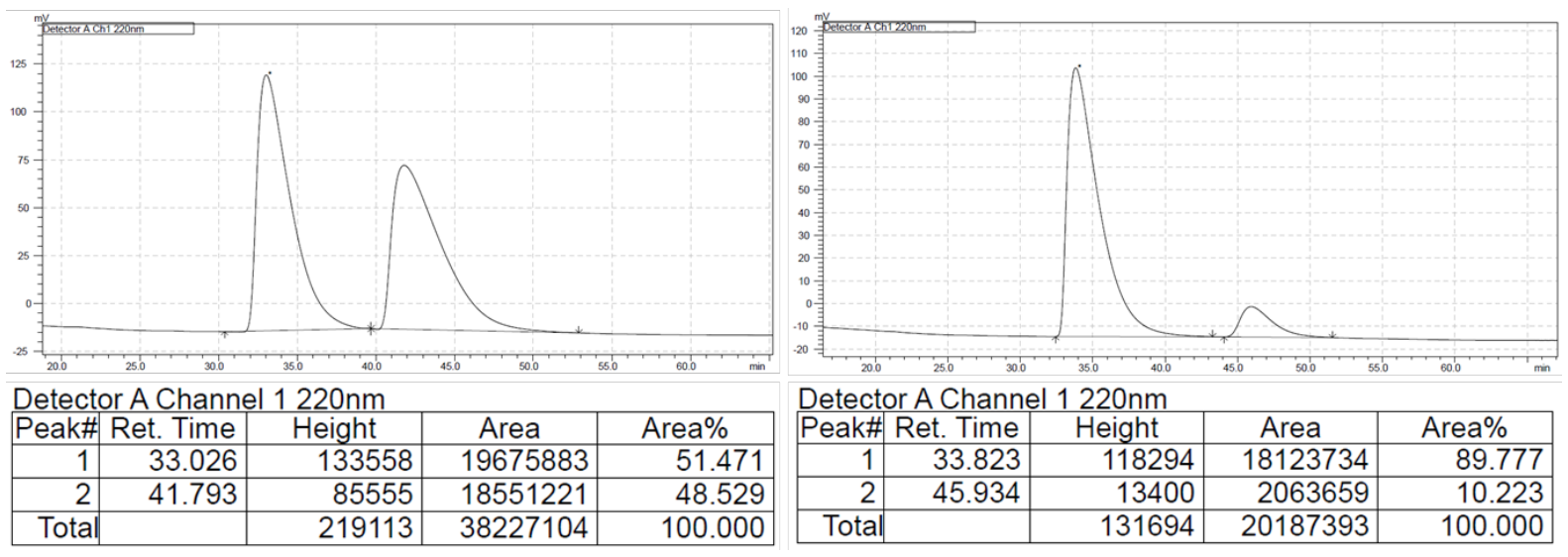

\begin{tabular}{|c|c|c|c|c|c|}
\hline Retention Time & Area & Area\% & Retention Time & Area & Area \% \\
\hline 33.026 & 19675883 & 51.471 & 33.823 & 18123734 & 89.777 \\
\hline 21.830 & 18551221 & 48.529 & 45.934 & 2063659 & 10.223 \\
\hline
\end{tabular}

6.2.11 $(R)-2-(2-((t-B u t y l d i m e t h y l s i l y l) o x y) e t h y l)-1-p h e n y l-3-(4,4,5,5-t e t r a m e t h y l-1,3,2-$ dioxaborolan-2-yl)but-3-en-1-one (2k)<smiles>CC(C)(O)C(CCO)C(=O)c1ccccc1</smiles>

Colorless oil; $38.7 \mathrm{mg}, 0.090 \mathrm{mmol}, 90 \%$ yield, 98:2 e.r.; IR (neat): 2928 (m), 1681 (m), 1359 (m), 1135 (m), 1218 (s), 832 (s), 791 (s), 687 (m) cm ${ }^{-1} ;{ }^{1}$ H NMR (400 MHz, CDCl $)$ ): $\delta 8.08-$ $7.92(\mathrm{~m}, 1 \mathrm{H}), 7.53-7.46(\mathrm{~m}, 1 \mathrm{H}), 7.45-7.38(\mathrm{~m}, 1 \mathrm{H}), 5.91(\mathrm{~d}, J=2.3 \mathrm{~Hz}, 1 \mathrm{H}), 5.67(\mathrm{~d}, J=2.6$ $\mathrm{Hz}, 1 \mathrm{H}), 4.47$ (t, $J=6.9 \mathrm{~Hz}, 1 \mathrm{H}), 3.62$ (qt, $J=10.1,6.3 \mathrm{~Hz}, 2 \mathrm{H}), 2.45-2.09$ (m, 1H), 2.08-1.77 $(\mathrm{m}, 1 \mathrm{H}), 1.27(\mathrm{~s}, 12 \mathrm{H}), 0.89(\mathrm{~s}, 9 \mathrm{H}), \delta 0.02(\mathrm{~s}, 3 \mathrm{H}),-0.01(\mathrm{~s}, 3 \mathrm{H}) ;{ }^{13} \mathrm{C}$ NMR (126 MHz, $\left.\mathbf{C D C l}_{3}\right): \delta 200.6,140.1$ (C-B bs), 136.8, 132.5, 131.4, 128.8, 128.2, 83.7, 61.0, 47.2, 35.3, 25.9, 24.8, 24.7, 18.2, -5.18, -5.24; ${ }^{11} \mathbf{B}$ NMR (160 MHz, $\left.\mathbf{C D C l}_{3}\right): \delta$ 29.9; HRMS (DART): Calcd for $\mathrm{C}_{24} \mathrm{H}_{39} \mathrm{BO}_{4} \mathrm{Si}_{1}[\mathrm{M}+\mathrm{H}]^{+}: 431.2789$, Found: 431.2801 ; specific rotation: $[\alpha]_{\mathrm{D}}{ }^{20}=$ $-26.8\left(c 1.0, \mathrm{CHCl}_{3}\right)$ for an enantiomerically enriched sample of 98:2 e.r.

Enantiomeric purity was determined by HPLC analysis of the pure product in comparison with authentic racemic material; Chiralcel AD-H column, 100: 0 hexanes $/ i-\mathrm{PrOH}, 0.5 \mathrm{~mL} / \mathrm{min}, 220$ $\mathrm{nm}$.
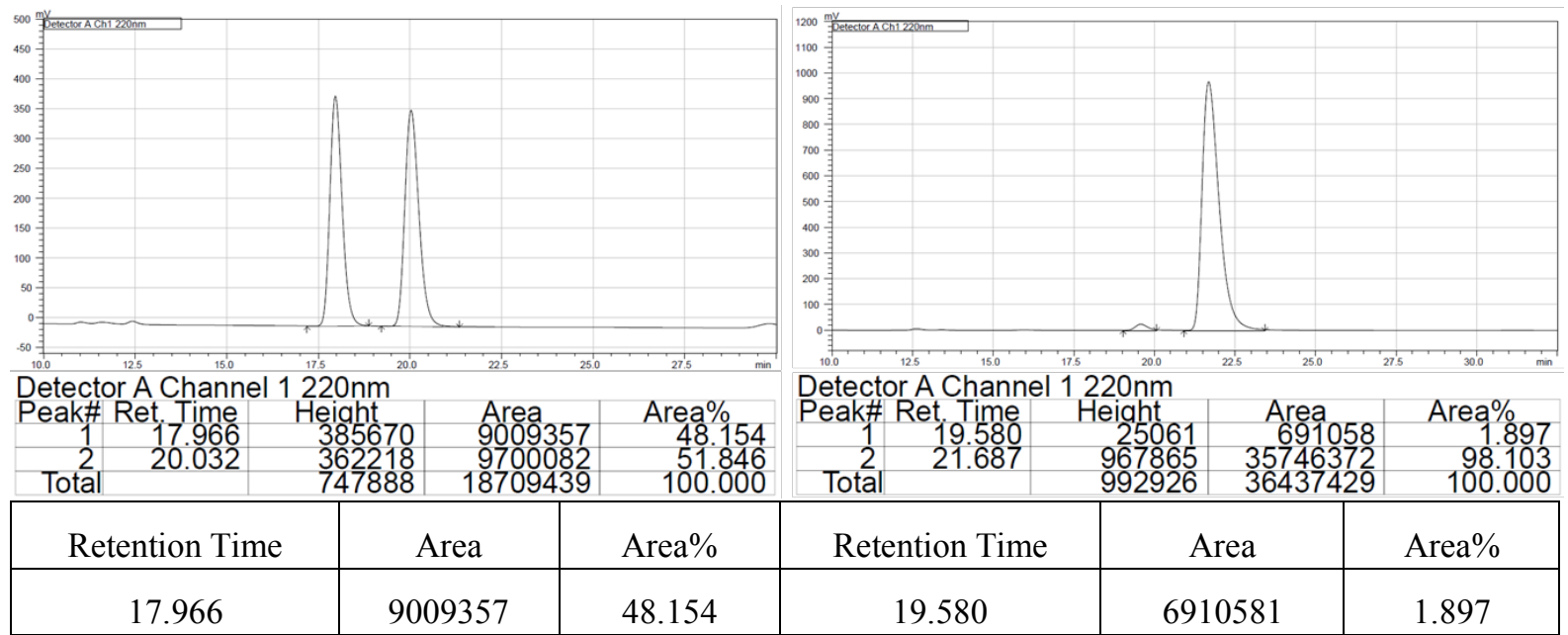


\begin{tabular}{|l|l|l|l|l|l|}
\hline 20.032 & 9700082 & 51.846 & 21.687 & 35746372 & 98.103 \\
\hline
\end{tabular}

6.2.12 (R)-2-Methyl-1-phenyl-3-(4,4,5,5-tetramethyl-1,3,2-dioxaborolan-2-yl)but-3-en1-one (2I)

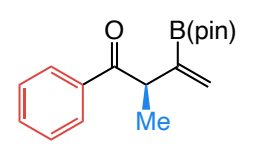

Colorless oil; $26.0 \mathrm{mg}, 0.091 \mathrm{mmol}, 91 \%$ yield, 99:1 e.r.; IR (neat): 2977 (w), 1684 (m), 1311 (s), 1135 (s), 835 (s), 688 (s) cm ${ }^{-1} ;{ }^{1} \mathbf{H}$ NMR (500 MHz, CDCl $): \delta 8.01-7.90$ (m, 2H), 7.55$7.45(\mathrm{~m}, 1 \mathrm{H}), 7.44-7.36(\mathrm{~m}, 2 \mathrm{H}), 5.90(\mathrm{~d}, J=2.5 \mathrm{~Hz}, 1 \mathrm{H}), 5.64$ (d, $J=2.5 \mathrm{~Hz}, 1 \mathrm{H}), 4.31$ (q, $J$ $=6.8 \mathrm{~Hz}, 1 \mathrm{H}), 1.32(\mathrm{~d}, J=6.8 \mathrm{~Hz}, 3 \mathrm{H}), 1.26(\mathrm{~s}, 12 \mathrm{H}) ;{ }^{13} \mathbf{C} \mathbf{N M R}\left(\mathbf{1 2 6} \mathbf{M H z}, \mathbf{C D C l}_{\mathbf{3}}\right): \delta 201.7$, $136.8,132.6,130.8,128.8,128.4,84.0,45.1,29.9,24.9,24.8,17.6$. The carbon bearing the boron atom could not be detected due to quadrupolar effects; ${ }^{11} \mathbf{B}$ NMR (160 MHz, $\left.\mathbf{C D C l}_{3}\right): \delta$ 30.0; HRMS (DART): Calcd for $\mathrm{C}_{17} \mathrm{H}_{24} \mathrm{BO}_{3}[\mathrm{M}+\mathrm{H}]^{+}$: 287.1819, Found: 287.1832; specific rotation: $[\alpha]_{\mathrm{D}}{ }^{20}=-68.8\left(c 1.0, \mathrm{CHCl}_{3}\right)$ for an enantiomerically enriched sample of $99: 1$ e.r.

Enantiomeric purity was determined by HPLC analysis in comparison with authentic racemic material; $(R, R)$-Whelk-O1 column, 99.9:0.1 hexanes/i-PrOH, 0.5 mL/min, $220 \mathrm{~nm}$.
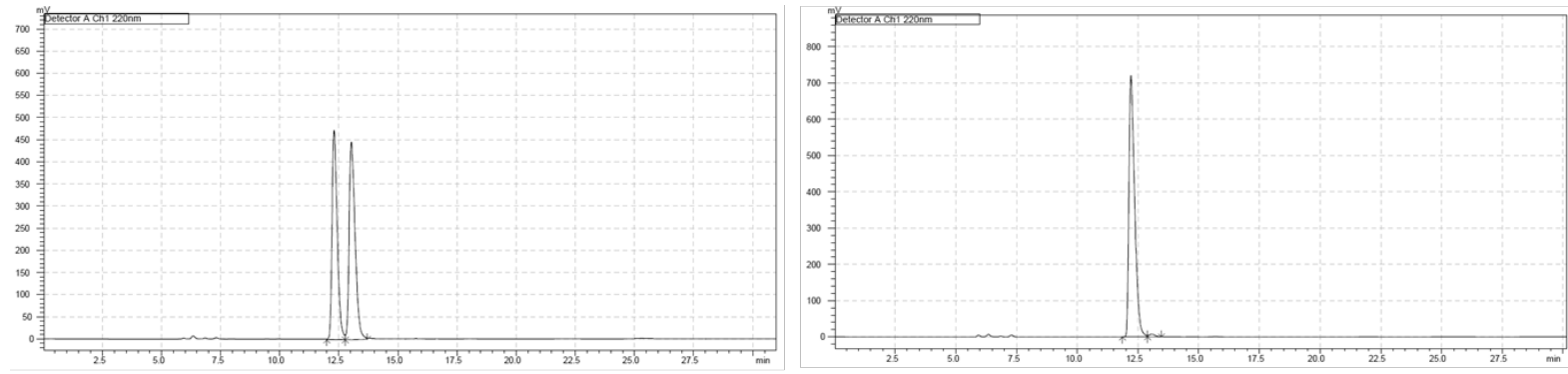

\begin{tabular}{|c|c|c|c|c|}
\hline \multicolumn{5}{|c|}{ Detector A Channel 1 220nm } \\
\hline Peak\# & et. Time & Height & Area & Area $\%$ \\
\hline 1 & 12.296 & 473299 & 7344225 & 48.047 \\
\hline 2 & 13.027 & 446910 & 7941263 & 51.953 \\
\hline Total & & 920210 & 15285489 & 100.000 \\
\hline
\end{tabular}

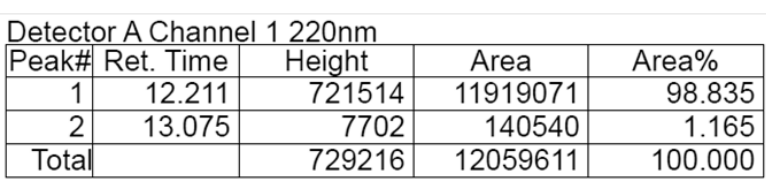

\begin{tabular}{|c|c|c|c|c|c|}
\hline Retention Time & Area & Area\% & Retention Time & Area & Area\% \\
\hline 12.296 & 7344225 & 48.047 & 12.211 & 11919071 & 98.835 \\
\hline 13.027 & 7941263 & 51.953 & 13.075 & 140540 & 1.165 \\
\hline
\end{tabular}

6.2.13 $(R)$-1-(4-Fluorophenyl)-2-methyl-3-(4,4,5,5-tetramethyl-1,3,2-dioxaborolan-2yl)but-3-en-1-one (2m; precursor to $[(S, R)-8 \mathrm{~d}]$; see Fig. 3c)

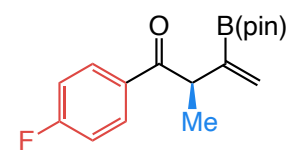

Modified procedure: $2.0 \mathrm{~mol} \%$ CuMes (18.2 mg, $0.100 \mathrm{mmol})$ and $2.2 \mathrm{~mol} \%$ phos-1 (59.6 $\mathrm{mg}, 0.110 \mathrm{mmol}$ ) was used.

Colorless oil; 1.40 g, 4.61 mmol, 92\% yield, 99:1 e.r.; IR (neat): 2976 (w), 1683 (s), 1597 (s), 1132 (m), 1223 (s), 1135 (s), 967 (m), 853 (m) cm ${ }^{-1}$, ${ }^{1} \mathbf{H}$ NMR (CDCl, 400 MHz): $\delta$ 8.02$7.95(\mathrm{~m}, 2 \mathrm{H}), 7.13-7.02(\mathrm{~m}, 2 \mathrm{H}), 5.91(\mathrm{~d}, J=2.3 \mathrm{~Hz}, 1 \mathrm{H}), 5.64(\mathrm{~d}, J=2.3 \mathrm{~Hz}, 1 \mathrm{H}), 4.26(\mathrm{q}, J$ $=6.8 \mathrm{~Hz}, 1 \mathrm{H}), 1.31(\mathrm{~d}, J=6.8 \mathrm{~Hz}, 3 \mathrm{H}), 1.26(\mathrm{~s}, 12 \mathrm{H}) ;{ }^{13} \mathbf{C} \mathbf{N M R}\left(\mathbf{C D C l}_{\mathbf{3}}, \mathbf{1 0 1} \mathbf{M H z}\right): \delta 200.1$, 
$165.5(\mathrm{~d}, J=253.7 \mathrm{~Hz}), 141.9(\mathrm{C}-\mathrm{B}, \mathrm{bs}), 133.2$ (d, $J=3.0 \mathrm{~Hz}), 131.4$ (d, $J=9.2 \mathrm{~Hz}), 131.1$, $115.5(\mathrm{~d}, J=21.6 \mathrm{~Hz}), 84.0,45.0,24.9,24.8,17.6 ;{ }^{\mathbf{1 9}} \mathbf{F} \mathbf{N M R}\left(\mathbf{C D C l}_{3}, \mathbf{3 7 6} \mathbf{~ M H z}\right): \delta-106.51$ $(\mathrm{tt}, J=8.3,5.4 \mathrm{~Hz}) ;{ }^{11} \mathbf{B}$ NMR $\left(\mathbf{C D C l}_{3}, \mathbf{1 6 0} \mathbf{M H z}\right): \delta$ 30.0; HRMS (DART): Calcd for $\mathrm{C}_{17} \mathrm{H}_{23} \mathrm{BO}_{3} \mathrm{~F}[\mathrm{M}+\mathrm{H}]^{+}: 305.1719$, Found: 305.1710 ; specific rotation: $[\alpha]_{\mathrm{D}}{ }^{20}=-88.9$ (c 1.0, $\mathrm{CHCl}_{3}$ ) for an enantiomerically enriched sample of 99:1 e.r.

Enantiomeric purity was determined by HPLC analysis in comparison with authentic racemic material; $(R, R)$-Whelk-O1 column, 99.9:0.1 hexanes/i-PrOH, 0.6 mL/min, $220 \mathrm{~nm}$.
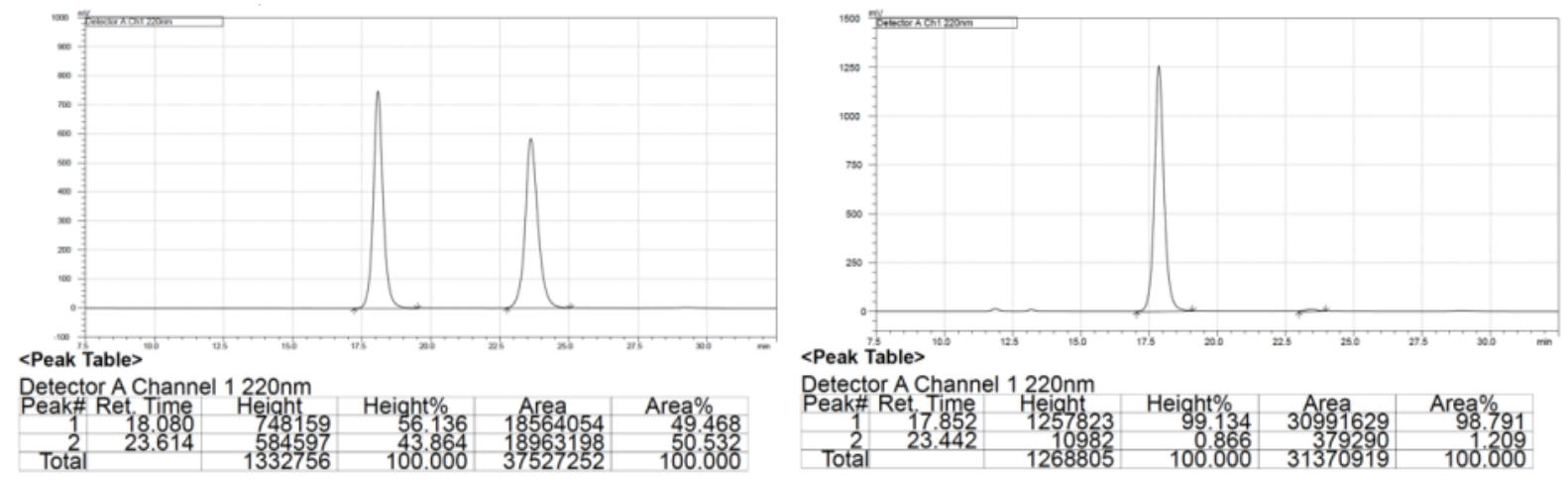

\begin{tabular}{|c|c|c|c|c|c|}
\hline Retention Time & Area & Area\% & Retention Time & Area & Area $\%$ \\
\hline 18.080 & 18564054 & 49.468 & 17.852 & 30991629 & 98.791 \\
\hline 23.614 & 18963198 & 50.532 & 23.442 & 379290 & 1.209 \\
\hline
\end{tabular}

\subsection{Reactions with $\alpha, \beta$-unsaturated nitriles}

\subsection{1 (R,E)-3-(2-((t-Butyldimethylsilyl)oxy)ethyl)-2-(4,4,5,5-tetramethyl-1,3,2-} dioxaborolan-2-yl)nona-1,5-dien-4-one (3a)

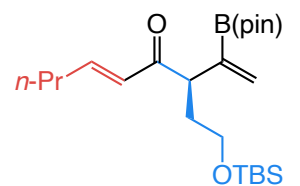

Colorless oil; 27.9 mg, 0.066 mmol, 66\% yield, 99:1 e.r.; IR (neat): 2957 (m), 2929 (m), 2857 (m), 1696 (m), 1670 (m), 1311 (s), 963 (m), 834 (s), 774 (s) $\mathrm{cm}^{-1} ;{ }^{1} \mathbf{H}$ NMR (600 MHz, $\left.\mathbf{C D C l}_{3}\right): \delta 6.87(\mathrm{dt}, J=15.6,6.9 \mathrm{~Hz}, 1 \mathrm{H}), 6.12(\mathrm{~d}, J=15.6 \mathrm{~Hz}, 1 \mathrm{H}), 5.92(\mathrm{~d}, J=2.8 \mathrm{~Hz}, 1 \mathrm{H})$, $5.65(\mathrm{~d}, J=2.8 \mathrm{~Hz}, 1 \mathrm{H}), 3.74(\mathrm{t}, J=6.9 \mathrm{~Hz}, 1 \mathrm{H}), 3.55$ (t, $J=6.4 \mathrm{~Hz}, 2 \mathrm{H}), 2.14(\mathrm{q}, \mathrm{J}=7.2 \mathrm{~Hz}$, $2 \mathrm{H}), 2.11-2.04(\mathrm{~m}, 1 \mathrm{H}), 1.81-1.73(\mathrm{~m}, 1 \mathrm{H}), 1.52-1.42(\mathrm{~m}, 2 \mathrm{H}), 1.26(\mathrm{~s}, 12 \mathrm{H}), 0.91(\mathrm{t}, J=7.4$ $\mathrm{Hz}, 2 \mathrm{H}), 0.88$ (s, 9H), 0.02 (s, 3H), 0.01 (s, 3H); $\left.{ }^{13} \mathbf{C ~ N M R ~ ( 1 5 1 ~ M H z , ~ C D C l} \mathbf{3}\right): \delta 200.5,147.0$, 139.7, 131.7, 129.9, 83.8, 61.1, 50.7, 34.6, 34.2, 26.1, 24.9, 24.8, 21.5, 18.4, 13.9, -5.15, -5.19; ${ }^{11}$ B NMR (192 MHz, CDCl 3 ): $\delta$ 29.3; HRMS (DART): Calcd for $\mathrm{C}_{23} \mathrm{H}_{44} \mathrm{BO}_{4} \mathrm{Si}[\mathrm{M}+\mathrm{H}]^{+}$: 423.3102, Found: 423.3119; specific rotation: $[\alpha]_{\mathrm{D}}{ }^{20}=-39.4$ (c 1.0, $\left.\mathrm{CHCl}_{3}\right)$ for an enantiomerically enriched sample of 99:1 e.r.

Enantiomeric purity was determined by HPLC analysis in comparison with authentic racemic material; Chiralcel OD-H column, 99.5:0.5 hexanes $/ i$-PrOH, $0.5 \mathrm{~mL} / \mathrm{min}, 220 \mathrm{~nm}$. 

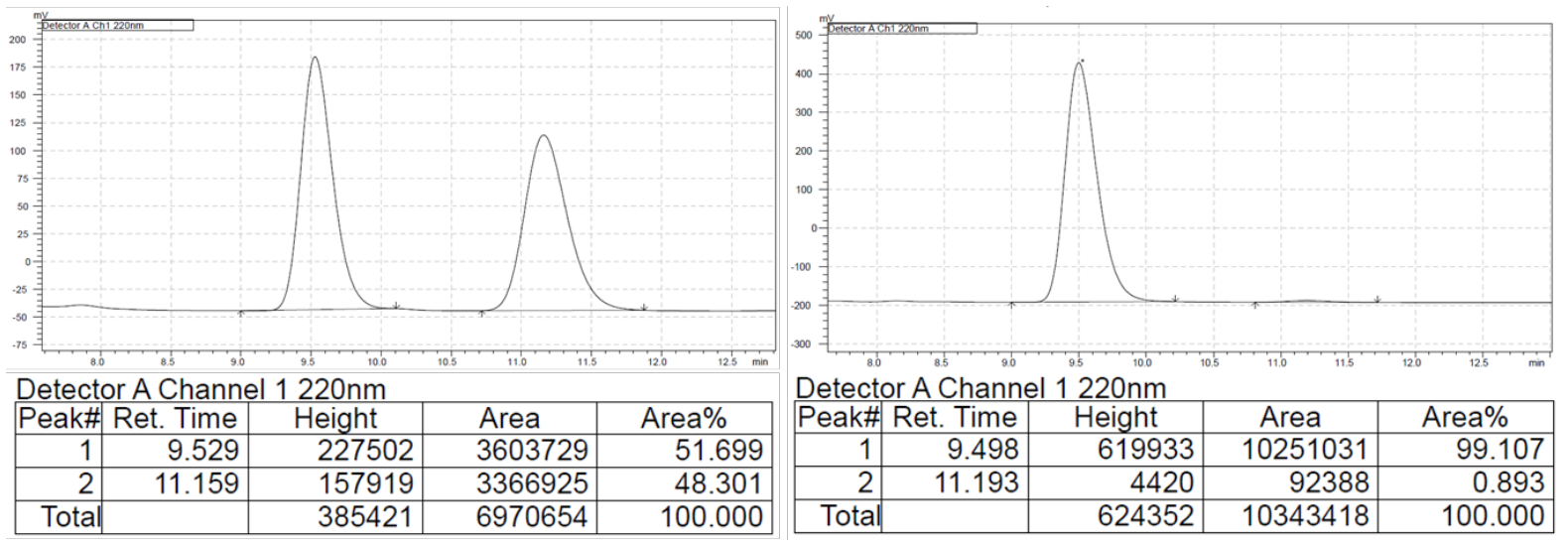

\begin{tabular}{|c|c|c|c|c|c|}
\hline Retention Time & Area & Area\% & Retention Time & Area & Area $\%$ \\
\hline 9.529 & 3603729 & 51.699 & 9.498 & 10251031 & 99.107 \\
\hline 11.159 & 3366925 & 48.301 & 11.193 & 92388 & 0.893 \\
\hline
\end{tabular}

6.3.2 $t$-Butyl $\quad(R, Z)-4-(4-(2-((t$-butyldimethylsilyl)oxy)ethyl)-3-oxo-5-(4,4,5,5tetramethyl-1,3,2-dioxaborolan-2-yl)hexa-1,5-dien-1-yl)piperidine-1-carboxylate (3b)

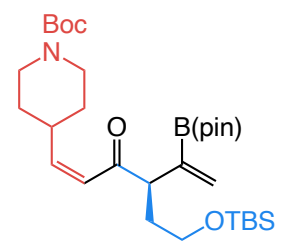

Colorless oil; $51.3 \mathrm{mg}, 0.091 \mathrm{mmol}, 91 \%$ yield, 99:1 e.r.; IR (neat): 2976 (m), 2929 (m), 2857 (m), 1695 (s), 1616 (w), 1470 (m), 1278 (m), 966 (m), 836 (m), 733 (w) cm ${ }^{-1}$; ${ }^{1}$ H NMR (600 MHz, CDCl $): \delta 6.12(\mathrm{~d}, J=11.4 \mathrm{~Hz}, 1 \mathrm{H}), 5.96(\mathrm{~d}, J=2.7 \mathrm{~Hz}, 1 \mathrm{H}), 5.78(\mathrm{dd}, J=11.4,9.3$ $\mathrm{Hz}, 1 \mathrm{H}), 5.66(\mathrm{~d}, J=2.7 \mathrm{~Hz}, 1 \mathrm{H}), 4.06(\mathrm{~s}, 2 \mathrm{H}), 3.62-3.43(\mathrm{~m}, 3 \mathrm{H}), 3.42-3.30(\mathrm{~m}, 1 \mathrm{H}), 2.75(\mathrm{~s}$, 2H), 2.14-2.02 (m, 1H), 1.77-1.58 (m, 3H), $1.44(\mathrm{~s}, 9 \mathrm{H}), 1.23(\mathrm{~s}, 12 \mathrm{H}), 0.87(\mathrm{~s}, 9 \mathrm{H}), 0.00(\mathrm{~s}$, 6H); ${ }^{13} \mathbf{C}$ NMR (151 MHz, $\left.\mathbf{C D C l}_{3}\right): \delta$ 201.2, 155.0, 150.8, 139.4, 132.4, 126.3, 83.8, 79.4, 77.4, 77.2, 77.0, 61.0, 54.4, 43.7, 35.8, 33.2, 31.3, 28.6, 26.1, 24.8, 18.4, $-5.16,-5.20 ;{ }^{11} \mathbf{B}$ NMR (192 MHz, CDCl $): \delta$ 29.3; HRMS (DART): Calcd for $\mathrm{C}_{30} \mathrm{H}_{55} \mathrm{BNO}_{6} \mathrm{Si}[\mathrm{M}+\mathrm{H}]^{+}$: 564.3886, Found: 564.3866; specific rotation: $\left.[\alpha]_{\mathrm{D}}{ }^{20}=-9.4(c) 1.0, \mathrm{CHCl}_{3}\right)$ for an enantiomerically enriched sample of 99:1 e.r.

Enantiomeric purity was determined by HPLC analysis in comparison with authentic racemic material; Chiralcel AD-H column, 99.9:0.1 hexanes $/ i-\mathrm{PrOH}, 0.5 \mathrm{~mL} / \mathrm{min}, 220 \mathrm{~nm}$.
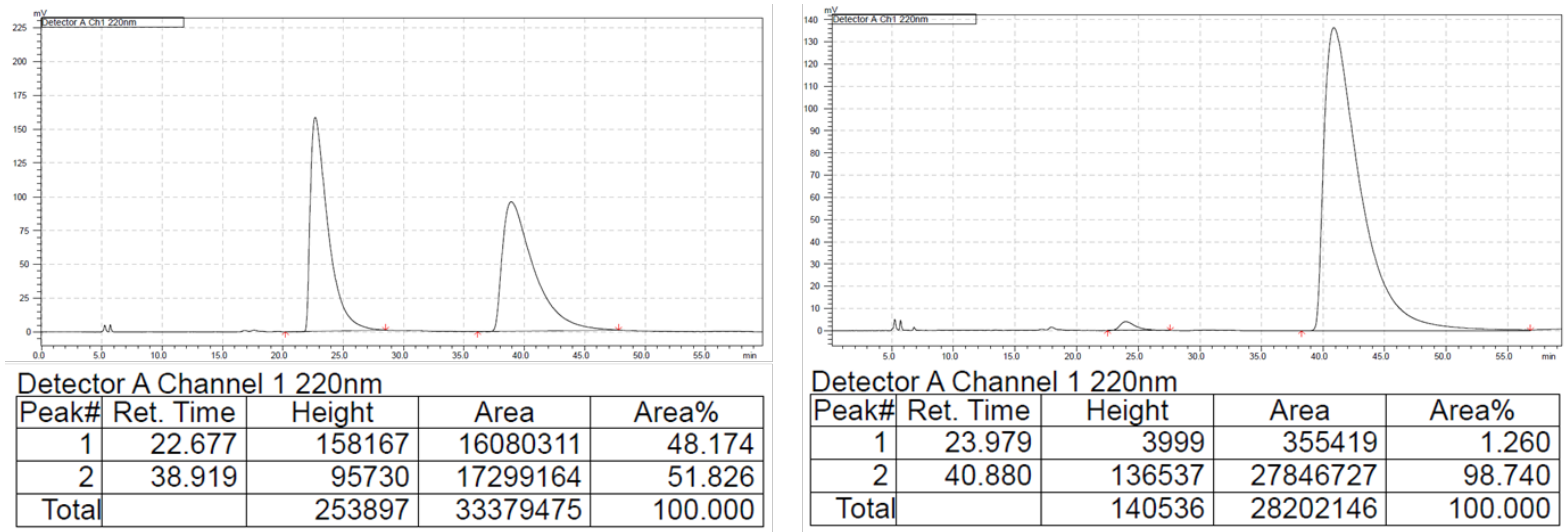


\begin{tabular}{|c|c|c|c|c|c|}
\hline Retention Time & Area & Area\% & Retention Time & Area & Area $\%$ \\
\hline 22.677 & 16080311 & 48.174 & 23.979 & 355419 & 1.260 \\
\hline 38.919 & 17299164 & 51.826 & 40.880 & 27846727 & 98.740 \\
\hline
\end{tabular}

6.3.3 (R,E)-2-Methyl-7-phenyl-4-(1-(4,4,5,5-tetramethyl-1,3,2-dioxaborolan-2yl)vinyl)hepta-1,6-dien-3-one (3c)

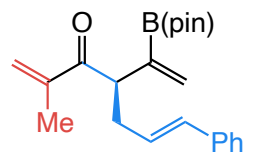

Modified procedure: 1.1 equiv. $\mathrm{B}_{2}$ (pin) 2 was (vs. 1.2 equiv.) was used to minimize reaction with the product (boryl conjugate addition).

Colorless oil; $27.1 \mathrm{mg}, 0.077$ mmol, 77\% yield, 99:1 e.r.; IR (neat): 3024 (w), 2975 (m), 2922 (m), 2849 (w), 1672 (s), 1577 (w), 1354 (s), 1311 (s), 963 (m), 691 (m) cm ${ }^{-1} ;{ }^{1}$ H NMR (600 MHz, CDCl $): \delta 7.33-7.29(\mathrm{~m}, 2 \mathrm{H}), 7.27-7.22(\mathrm{~m}, 2 \mathrm{H}), 7.17-7.13(\mathrm{~m}, 1 \mathrm{H}), 6.37(\mathrm{~d}, J=16.0$ $\mathrm{Hz}, 1 \mathrm{H}), 6.15(\mathrm{dt}, J=16.0,7.2 \mathrm{~Hz}, 1 \mathrm{H}), 6.00(\mathrm{~s}, 1 \mathrm{H}), 5.94(\mathrm{~s}, 1 \mathrm{H}), 5.71-5.62(\mathrm{~m}, 2 \mathrm{H}), 4.14(\mathrm{t}$, $J=7.2 \mathrm{~Hz}, 1 \mathrm{H}), 2.73-2.66(\mathrm{~m}, 1 \mathrm{H}), 2.49-2.40(\mathrm{~m}, 1 \mathrm{H}), 1.84(\mathrm{~d}, J=1.4 \mathrm{~Hz}, 3 \mathrm{H}), 1.22(\mathrm{~s}, 6 \mathrm{H})$, $1.21(\mathrm{~s}, 6 \mathrm{H}) ;{ }^{13} \mathbf{C}$ NMR (151 MHz, $\left.\mathbf{C D C l}_{3}\right): \delta 201.8,144.2,140.4(\mathrm{C}-\mathrm{B}$ bs), 137.9, 131.5, $131.31,131.29,128.6,128.5,127.0,126.2,124.9,124.9,84.0,49.6,36.4,24.9,24.8,18.3 ;{ }^{11} \mathbf{B}$ NMR (192 MHz, CDCl $)$ ): $\delta$ 29.7; HRMS (DART): Calcd for $\mathrm{C}_{22} \mathrm{H}_{30} \mathrm{BO}_{3}[\mathrm{M}+\mathrm{H}]^{+}: 353.2283$ Found: 353.2267 ; specific rotation: $[\alpha]_{\mathrm{D}}{ }^{20}=-108.6\left(c\right.$ 1.0, $\left.\mathrm{CHCl}_{3}\right)$ for an enantiomerically enriched sample of 99:1 e.r.

Enantiomeric purity was determined by HPLC analysis in comparison with authentic racemic material; $(R, R)$-Whelk-O1 column, 99.5:0.5 hexanes/i-PrOH, $0.5 \mathrm{~mL} / \mathrm{min}, 254 \mathrm{~nm}$.
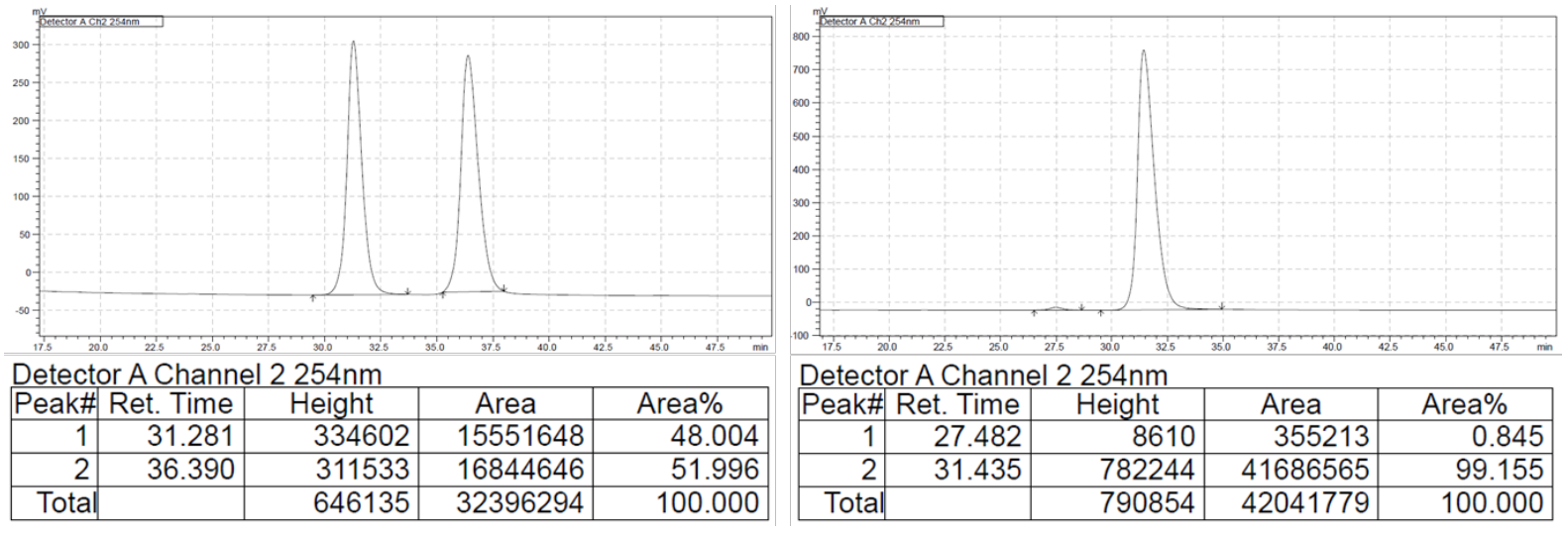

\begin{tabular}{|c|c|c|c|c|c|}
\hline Retention Time & Area & Area\% & Retention Time & Area & Area $\%$ \\
\hline 31.281 & 15551648 & 48.004 & 27.482 & 355213 & 0.845 \\
\hline 36.390 & 16844646 & 51.996 & 31.435 & 41686565 & 99.155 \\
\hline
\end{tabular}




\subsection{Reactions with alkyl nitriles}

6.4.1 (R)-7-Bromo-3-(2-((t-butyldimethylsilyl)oxy)ethyl)-2-(4,4,5,5-tetramethyl-1,3,2dioxaborolan-2-yl)hept-1-en-4-one (4a)<smiles>COCCC(C(C)=O)C(=O)CCCBr</smiles>

Modified procedure: reaction was quenched at $-40{ }^{\circ} \mathrm{C}$ to avoid decomposition.

Colorless oil, $39.0 \mathrm{mg}, 0.082 \mathrm{mmol}, 82 \%$ yield, 99:1 e.r.; IR (neat): 2955 (m), 2928 (m), 2856 (m), 1712 (m), 1141 (s), 835 (m), 776 (w) cm ${ }^{-1}$; ${ }^{1} \mathbf{H}$ NMR (600 MHz, $\left.\mathbf{C D C l}_{3}\right): \delta 5.98$ (d, $J=$ $2.7 \mathrm{~Hz}, 1 \mathrm{H}), 5.68(\mathrm{~d}, J=2.7 \mathrm{~Hz}, 1 \mathrm{H}), 3.61-3.45(\mathrm{~m}, 3 \mathrm{H}), 3.46-3.36(\mathrm{~m}, 2 \mathrm{H}), 2.72-2.63(\mathrm{~m}$, $1 \mathrm{H}), 2.62-2.53(\mathrm{~m}, 1 \mathrm{H}), 2.14-2.04(\mathrm{~m}, 3 \mathrm{H}), 1.76-1.66(\mathrm{~m}, 1 \mathrm{H}), 1.26(\mathrm{~s}, 12 \mathrm{H}), 0.88(\mathrm{~s}, 9 \mathrm{H})$, 0.02 (s, 6H); ${ }^{13} \mathbf{C}$ NMR (151 MHz, CDCl $)$ ): $\delta$ 209.9, 139.2 (C-B bs), 132.4, 83.9, 61.0, 53.8, 39.9, 33.7, 33.5, 26.9, 26.1, 24.92, 24.87, 18.4, -5.2; ${ }^{11} \mathbf{B}$ NMR (192 MHz, $\left.\mathbf{C D C l}_{3}\right): \delta$ 29.3; HRMS (ESI): Calcd for $\mathrm{C}_{21} \mathrm{H}_{41} \mathrm{BrBO}_{4} \mathrm{Si}$ : 475.2051, Found: 475.2052; specific rotation: $[\alpha]_{\mathrm{D}}^{20}=-42.6\left(c 1.0, \mathrm{CHCl}_{3}\right)$ for an enantiomerically enriched sample of 99:1 e.r.

Enantiomeric purity was determined by HPLC analysis in comparison with authentic racemic material; Chiralcel OD-H column, 100:0 hexanes $/ i-\mathrm{PrOH}, 0.5 \mathrm{~mL} / \mathrm{min}, 220 \mathrm{~nm}$.
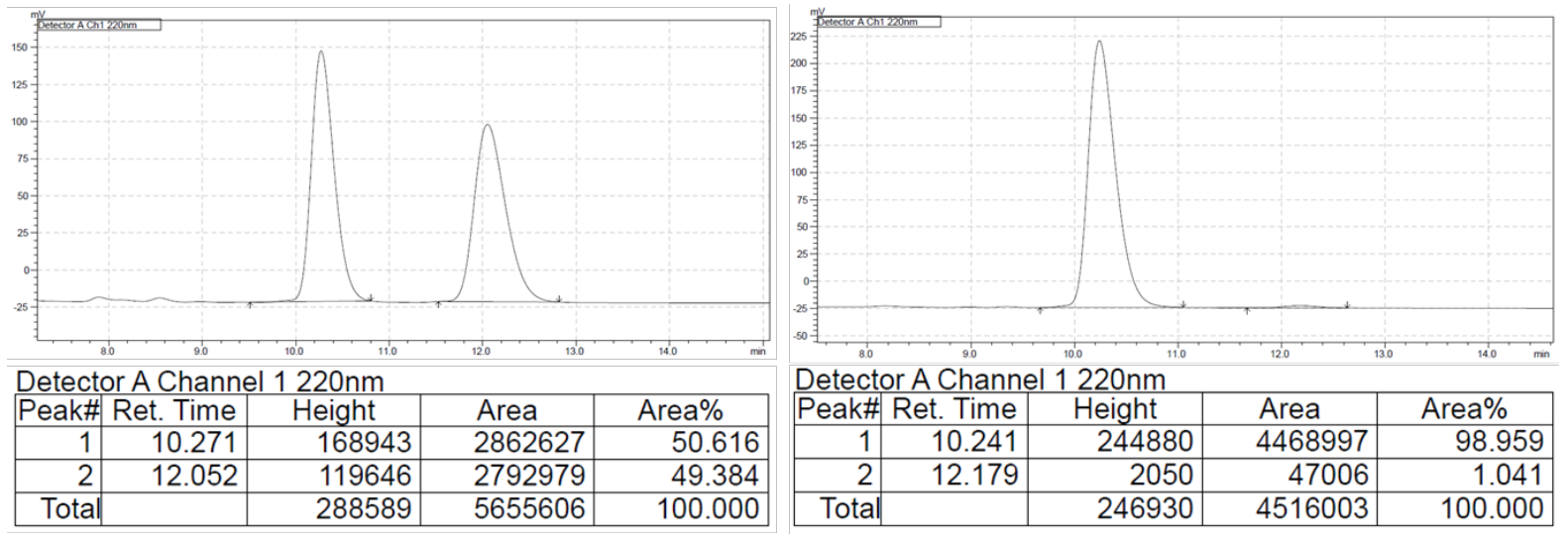

\begin{tabular}{|c|c|c|c|c|c|}
\hline Retention Time & Area & Area\% & Retention Time & Area & Area\% \\
\hline 10.271 & 2862627 & 50.616 & 10.241 & 4468997 & 98.959 \\
\hline 12.052 & 2792979 & 49.384 & 12.179 & 47006 & 1.041 \\
\hline
\end{tabular}

6.4.2 (R)-3-(2-((t-Butyldimethylsilyl)oxy)ethyl)-2-(4,4,5,5-tetramethyl-1,3,2dioxaborolan-2-yl)nona-1,8-dien-4-one (4b)

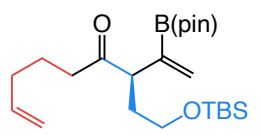

Colorless oil; $37.6 \mathrm{mg}, 0.089$ mmol, 89\% yield, 98:2 e.r.; IR (neat): 2954 (m), 2928 (m), 2856 (m), 1712 (m), $1312(\mathrm{~m}), 1140$ (s), 833 (s), 774 (s) cm ${ }^{-1} ;{ }^{1} \mathbf{H}$ NMR (500 MHz, CDCl 3 ): $\delta 5.94$ $(\mathrm{d}, J=2.7 \mathrm{~Hz}, 1 \mathrm{H}), 5.75$ (ddt, $J=17.0,10.2,6.7 \mathrm{~Hz}, 1 \mathrm{H}), 5.65(\mathrm{~d}, J=2.7 \mathrm{~Hz}, 1 \mathrm{H}), 5.03-4.88$ (m, 2H), 3.59-3.44 (m, 3H), 2.54-2.30 (m, 2H), 2.11-1.93 (m, 3H), 1.77-1.56 (m, 3H), 1.25

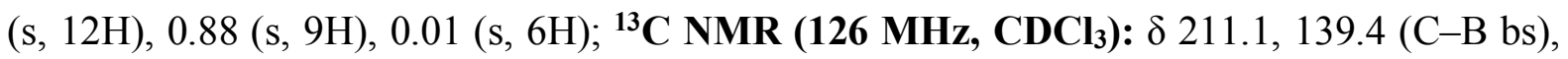
$138.4,132.0,115.1,83.8,61.1,53.5,41.2,33.6,33.3,26.1,24.9,23.1,18.4,-5.2 ;{ }^{11} \mathbf{B}$ NMR 
(160 MHz, $\mathbf{C D C l}_{3}$ ): $\delta$ 29.7; HRMS (DART): Calcd for $\mathrm{C}_{23} \mathrm{H}_{44} \mathrm{BO}_{4} \mathrm{Si}[\mathrm{M}+\mathrm{H}]^{+}:$423.3102, Found: 423.3111 ; specific rotation: $[\alpha]_{\mathrm{D}}{ }^{20}=-47.2\left(c\right.$ 1.0, $\left.\mathrm{CHCl}_{3}\right)$ for an enantiomerically enriched sample of 98:2 e.r.

Enantiomeric purity was determined by HPLC analysis in comparison with authentic racemic material; Chiralcel AD-H column, 100:0 hexanes $/ i-\mathrm{PrOH}, 0.5 \mathrm{~mL} / \mathrm{min}, 254 \mathrm{~nm}$.
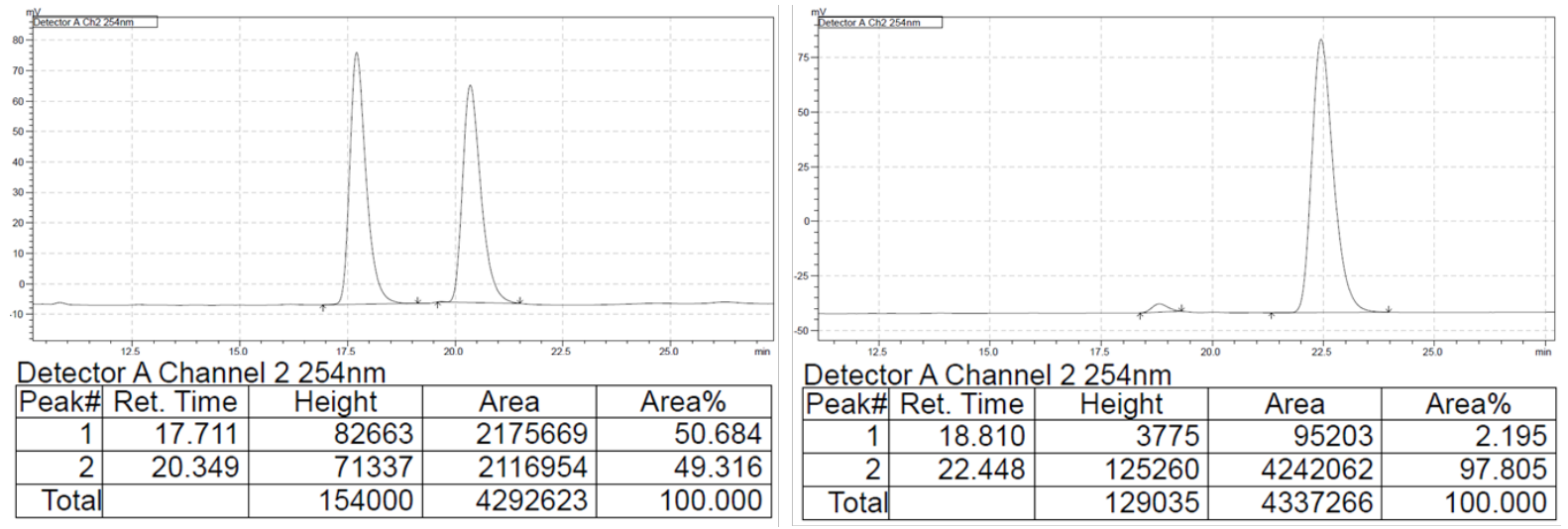

\begin{tabular}{|c|c|c|c|c|c|}
\hline Retention Time & Area & Area\% & Retention Time & Area & Area\% \\
\hline 17.711 & 2175669 & 50.684 & 18.810 & 95203 & 2.195 \\
\hline 20.349 & 2116954 & 49.316 & 22.448 & 4242062 & 97.805 \\
\hline
\end{tabular}

6.4.3 (R)-3-Cyclohexyl-2-(4,4,5,5-tetramethyl-1,3,2-dioxaborolan-2-yl)non-1-en-8-yn4-one (4c)<smiles>C=CCCC(=O)C(Cl)C(=O)O</smiles>

Colorless oil; 29.9 mg, 0.087 mmol, 87\% yield, >99:1 e.r.; IR (neat): 3270 (w), 2980 (s), 2922 (m), 2151 (w), 1958 (w), 1703 (m), 1382 (m), 1144 (s), 966 (m) cm ${ }^{-1} ;{ }^{1}$ H NMR (500 MHz, $\left.\mathbf{C D C l}_{3}\right): \delta 5.97(\mathrm{~d}, J=2.7 \mathrm{~Hz}, 1 \mathrm{H}), 5.72(\mathrm{~d}, J=2.7 \mathrm{~Hz}, 1 \mathrm{H}), 2.63-2.52(\mathrm{~m}, 1 \mathrm{H}), 2.49-2.40(\mathrm{~m}$, 1H), 2.24-2.09 (m, 2H), 2.04-1.86 (m, 2H), 1.82-1.54 (m, 7H), 1.34-1.04 (m, 15H), 0.96$0.74(\mathrm{~m}, 2 \mathrm{H}) ;{ }^{13} \mathbf{C}$ NMR (126 MHz, $\left.\mathbf{C D C l}_{3}\right): \delta 210.6,138.4$ (C-B bs), 132.7, 84.0, 68.8, 62.9, 41.9, 39.7, 32.2 , 30.6, 26.6, 26.4, 26.2, 24.8, 22.5, 17.9; ${ }^{11} \mathbf{B}$ NMR (160 MHz, $\left.\mathbf{C D C l}_{3}\right): \delta 29.9$; HRMS (DART): Calcd for $\mathrm{C}_{21} \mathrm{H}_{34} \mathrm{BO}_{3}[\mathrm{M}+\mathrm{H}]^{+}$: 345.2596, Found: 345.2584; specific rotation: $[\alpha]_{\mathrm{D}}{ }^{20}=-79.4\left(c 1.0, \mathrm{CHCl}_{3}\right)$ for an enantiomerically enriched sample of $>99: 1$ e.r. Enantiomeric purity was determined by HPLC analysis in comparison with authentic racemic material; $(R, R)$-Whelk-O1 column, 99.9:0.1 hexanes/i-PrOH, 0.5 mL/min, $210 \mathrm{~nm}$. 

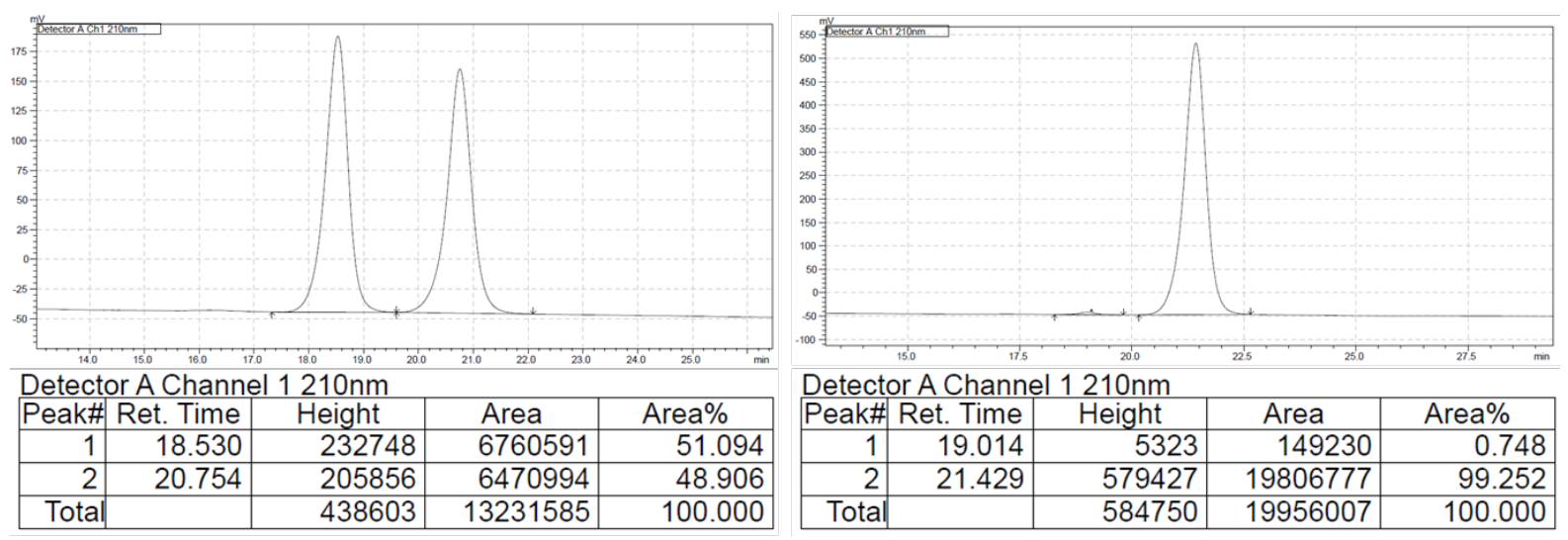

\begin{tabular}{|c|c|c|c|c|c|}
\hline Retention Time & Area & Area\% & Retention Time & Area & Area\% \\
\hline 18.530 & 6760591 & 51.094 & 19.014 & 149230 & 0.748 \\
\hline 20.754 & 6470994 & 48.906 & 21.429 & 19806777 & 99.252 \\
\hline
\end{tabular}

6.4.4 Methyl (R)-5-(2-((t-butyldimethylsilyl)oxy)ethyl)-4-oxo-6-(4,4,5,5-tetramethyl1,3,2-dioxaborolan-2-yl)hept-6-enoate (4d)<smiles>C=C(O)C(CCC(C)=O)C(=O)CCCC</smiles>

The aforementioned general procedure was modified. A two-necked flask connected to a $\mathrm{N}_{2}$ inlet was charged with $\left[\mathrm{CuF}\left(\mathrm{PPh}_{3}\right)_{3} \cdot 2 \mathrm{EtOH}\right](192.3 \mathrm{mg}, 0.20 \mathrm{mmol})$, phos-1 (119.4 mg, 0.22 $\mathrm{mmol})$ and THF $(20 \mathrm{~mL})$. Anhydrous $\mathrm{MeOH}(352 \mathrm{mg}, 11.0 \mathrm{mmol})$ was added by syringe and the mixture was kept at $22{ }^{\circ} \mathrm{C}$ for $10 \mathrm{~min}$. At this time, the mixture was allowed to cool to $0{ }^{\circ} \mathrm{C}$ and charged with a solution of allyl cyanide $(670 \mathrm{mg}, 10.0 \mathrm{mmol})$, allene $t$ butyldimethyl(penta-3,4-dien-1-yloxy)silane $(2.38 \mathrm{~g}, 1.2 \mathrm{mmol})$ and $\mathrm{B}_{2}(\text { pin) })_{2}(3.05 \mathrm{~g}, 1.2$ $\mathrm{mmol})$ in THF $(80 \mathrm{~mL})$. The mixture was allowed to stir for $1 \mathrm{~h}$ at $0{ }^{\circ} \mathrm{C}$ after which it was allowed to warm to $22^{\circ} \mathrm{C}$ and stir at this temperature for $2 \mathrm{~h}$. After full conversion was observed (TLC analysis), the reaction was quenched by the addition of a saturated aqueous solution of $\mathrm{NH}_{4} \mathrm{Cl}(50 \mathrm{~mL})$. Water $(50 \mathrm{~mL})$ was added and the aqueous layer was washed with $\mathrm{Et}_{2} \mathrm{O}(3 \times$ $40 \mathrm{~mL}$ ). The combined organic layers were dried over $\mathrm{MgSO}_{4}$, filtered and concentrated in vacuo to afford pale-yellow oil, purification of which by silica gel chromatography $(20: 1 \rightarrow$ 10:1 hexanes:EtOAc; $\mathrm{R}_{f}=0.40$ (5:1 hexanes:EtOAc)) afforded $\mathbf{4 d}$ as colorless oil (2.96 g, 7.5 mmol, 75\% yield).

Colorless oil; $4.11 \mathrm{~g}, 9.331 \mathrm{mmol}, 89 \%$ yield, 98:2 e.r.; IR (neat): 2980 (w), 2928 (w), 1742 (m), 1714 (m), 1358 (m), 1140 (s), 1098(s), 833 (s), 774 (s) cm ${ }^{-1} ;{ }^{1}$ H NMR (600 MHz, CDCl $\left._{3}\right): \delta 5.97(\mathrm{~d}, J=2.7 \mathrm{~Hz}, 1 \mathrm{H}), 5.68(\mathrm{~d}, J=2.7 \mathrm{~Hz}, 1 \mathrm{H}), 3.65(\mathrm{~s}, 3 \mathrm{H}), 3.59-3.47(\mathrm{~m}, 3 \mathrm{H})$, 2.86-2.67 (m, 2H), 2.60-2.45 (m, 2H), 2.13-2.02 (m, 1H), 1.76-1.67 (m, 1H), $1.25(\mathrm{~s}, 12 \mathrm{H})$, 0.87 (s, 9H), 0.01 (s, 6H); ${ }^{13} \mathbf{C}$ NMR (151 MHz, $\left.\mathbf{C D C l}_{3}\right): \delta 209.2,139.2$ (C-B bs), 173.4, 132.3, 83.9, 61.0, 53.5, 51.8, 36.6, 33.5, 28.2, 26.1, 24.9, 18.4, -5.2; ${ }^{11}$ B NMR (192 MHz, $\mathbf{C D C l}_{3}$ ): $\delta$ 29.4; HRMS (DART): Calcd for $\mathrm{C}_{22} \mathrm{H}_{42} \mathrm{BO}_{6} \mathrm{Si}[\mathrm{M}+\mathrm{H}]^{+}:$441.2844, Found: 
441.2859; specific rotation: $[\alpha]_{\mathrm{D}^{20}}=-47.8\left(c 1.0, \mathrm{CHCl}_{3}\right)$ for an enantiomerically enriched sample of 98:2 e.r.

Enantiomeric purity was determined by HPLC analysis in comparison with authentic racemic material; Chiralcel OD-H column, 99.5:0.5 hexanes $/ i$-PrOH, $0.5 \mathrm{~mL} / \mathrm{min}, 220 \mathrm{~nm}$.
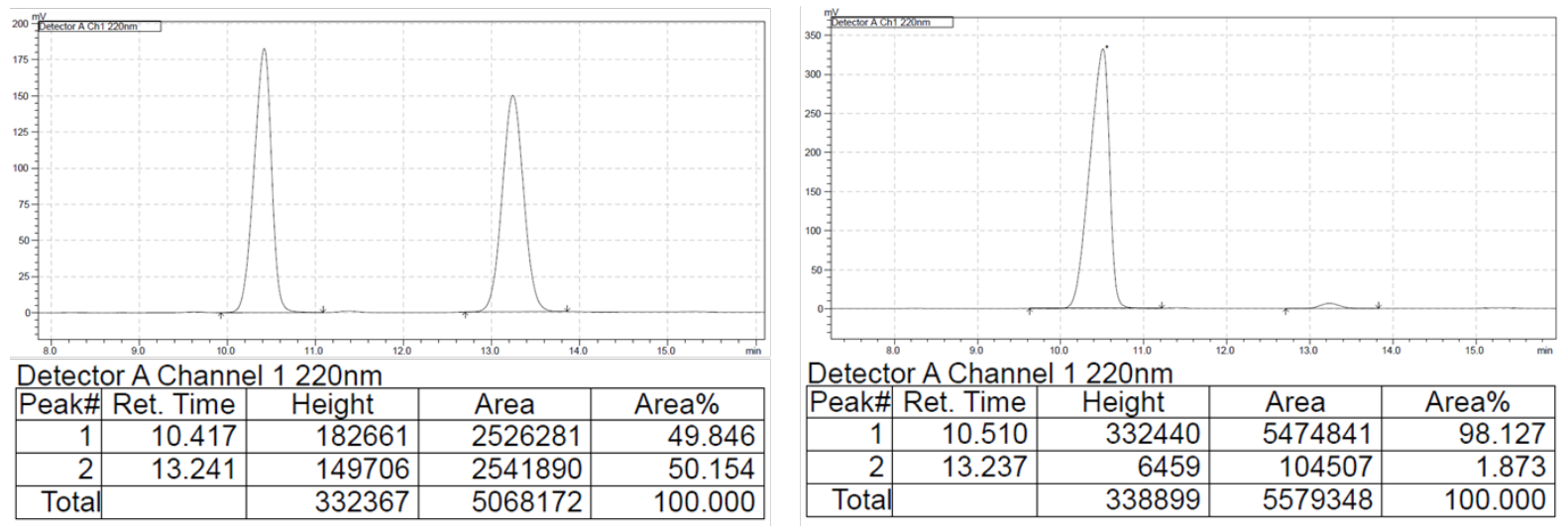

\begin{tabular}{|c|c|c|c|c|c|}
\hline Retention Time & Area & Area\% & Retention Time & Area & Area\% \\
\hline 10.417 & 2526281 & 49.846 & 10.510 & 5474841 & 98.127 \\
\hline 13.241 & 2541890 & 50.154 & 13.237 & 104507 & 1.873 \\
\hline
\end{tabular}

6.4.5 (R,E)-10-Azido-1-phenyl-4-(1-(4,4,5,5-tetramethyl-1,3,2-dioxaborolan-2yl)vinyl)dec-1-en-5-one (4e)

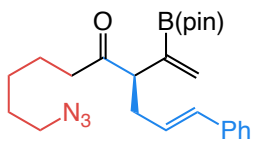

The general procedure (see above) was modified: the reaction was run at $22{ }^{\circ} \mathrm{C}$ for 20 min with $3.5 \mathrm{mg}$ of $\mathrm{MeOH}(0.11 \mathrm{mmol})$ and $38.1 \mathrm{mg}$ of $\mathrm{B}_{2}(\text { pin })_{2}(0.15 \mathrm{mmol})$. The reaction was quenched by addition of a saturated solution of aqueous $\mathrm{NH}_{4} \mathrm{Cl}$.

Colorless oil; 32.1 mg, 0.076 mmol, 76\% yield, 98:2 e.r.; IR (neat): 2977 (w), 2931 (w), 2094 (s), 1711 (m), 1316 (s), 1138 (s), 965 (m), 693 (m) cm ${ }^{-1} ;{ }^{1}$ H NMR (500 MHz, CDCl $): \delta 7.32-$ 7.23 (m, 4H), 7.20-7.15 (m, 1H), 6.37 (dd, $J=15.7,1.5 \mathrm{~Hz}, 1 \mathrm{H}), 6.12$ (dt, $J=15.7,7.2 \mathrm{~Hz}$, $1 \mathrm{H}), 6.01(\mathrm{~d}, J=2.5 \mathrm{~Hz}, 1 \mathrm{H}), 5.71(\mathrm{~d}, J=2.5 \mathrm{~Hz}, 1 \mathrm{H}), 3.47(\mathrm{t}, J=7.2 \mathrm{~Hz}, 1 \mathrm{H}), 3.21(\mathrm{t}, J=7.2$ $\mathrm{Hz}, 2 \mathrm{H}), 2.76-2.66(\mathrm{~m}, 1 \mathrm{H}), 2.53-2.33(\mathrm{~m}, 3 \mathrm{H}), 1.61-1.49(\mathrm{~m}, 4 \mathrm{H}), 1.35-1.29(\mathrm{~m}, 2 \mathrm{H}), 1.24$ $(\mathrm{s}, 12 \mathrm{H}) ;{ }^{13} \mathbf{C}$ NMR (126 MHz, $\left.\mathbf{C D C l}_{3}\right): \delta 210.3,137.7,132.4,131.7,128.5,128.4,127.1$, 126.1, 84.0, 56.8, 51.4, 42.0, 34.6, 28.8, 26.4, 24.9, 24.9, 23.3. The carbon bearing the boron atom could not be detected due to quadrupolar effects; ${ }^{11} \mathbf{B} \mathbf{N M R}\left(\mathbf{1 6 0} \mathbf{M H z}, \mathbf{C D C l}_{3}\right): \delta$ 29.8; HRMS (DART): Calcd for $\mathrm{C}_{24} \mathrm{H}_{35} \mathrm{~N}_{3} \mathrm{BO}_{3}[\mathrm{M}+\mathrm{H}]^{+}$: 424.2766, Found: 424.2756; specific rotation: $[\alpha]_{\mathrm{D}}{ }^{20}=-62.0\left(c 1.0, \mathrm{CHCl}_{3}\right)$ for an enantiomerically enriched sample of 98:2 e.r.

Enantiomeric purity was determined by HPLC analysis in comparison with authentic racemic material; Chiralcel OD-H column, 99.0:1.0 hexanes $/ i$-PrOH, $0.5 \mathrm{~mL} / \mathrm{min}, 220 \mathrm{~nm}$. 

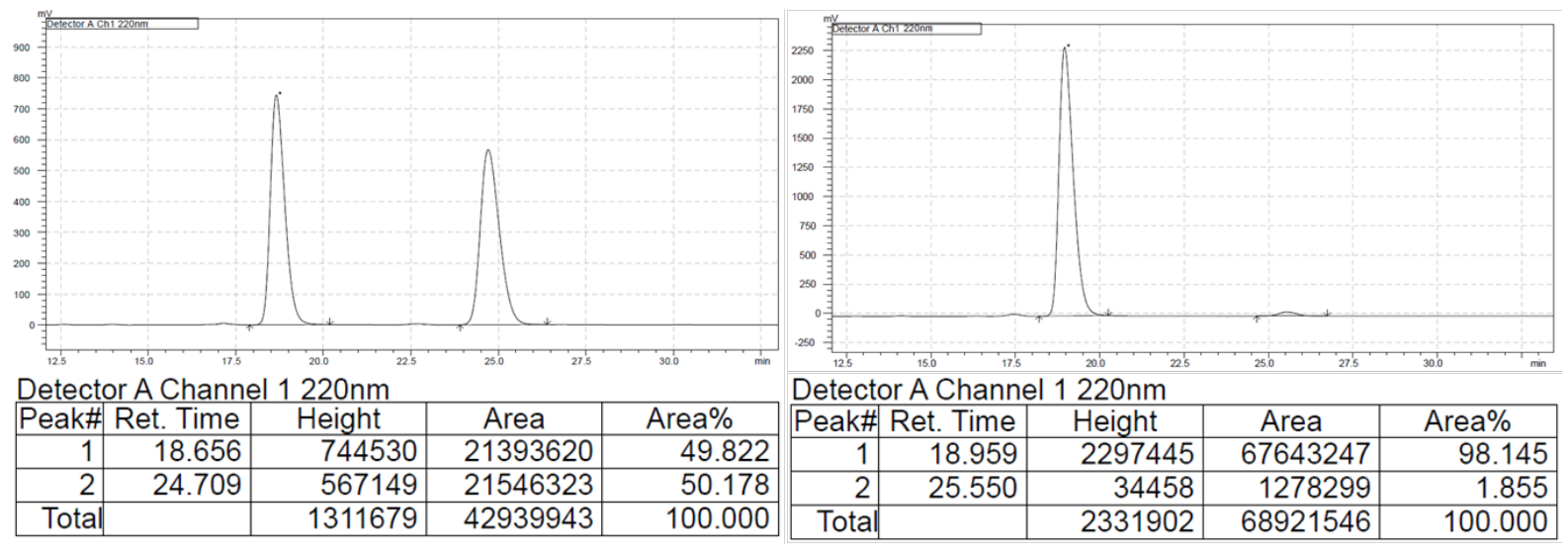

\begin{tabular}{|c|c|c|c|c|c|}
\hline Retention Time & Area & Area\% & Retention Time & Area & Area $\%$ \\
\hline 18.656 & 21393620 & 49.822 & 18.959 & 67643247 & 98.145 \\
\hline 24.709 & 21546323 & 50.178 & 25.550 & 1278299 & 1.855 \\
\hline
\end{tabular}

6.4.6 t-Butyl (R)-5-oxo-10-(4-phenyl-1H-1,2,3-triazol-1-yl)-4-(1-(4,4,5,5-tetramethyl1,3,2-dioxaborolan-2-yl)vinyl)decanoate (4f)

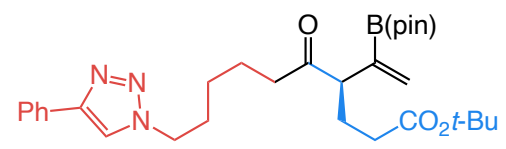

Colorless oil; $42.4 \mathrm{mg}, 0.0789$ mmol, 79\% yield, 99:1 e.r.; IR (neat): 2974, 2932 (m), 1722

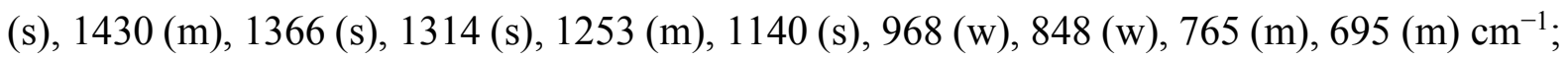

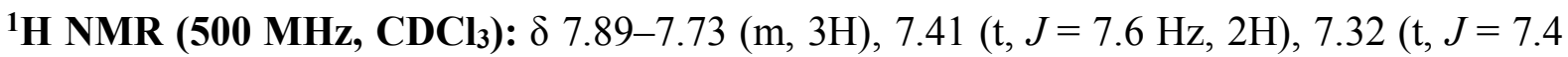
$\mathrm{Hz}, 1 \mathrm{H}), 5.97$ (d, $J=2.5 \mathrm{~Hz}, 1 \mathrm{H}), 5.65(\mathrm{~d}, J=2.5 \mathrm{~Hz}, 1 \mathrm{H}), 4.38(\mathrm{t}, J=7.1 \mathrm{~Hz}, 2 \mathrm{H}), 3.29(\mathrm{t}, J$ $=7.0 \mathrm{~Hz}, 1 \mathrm{H}), 2.49-2.33(\mathrm{~m}, 2 \mathrm{H}), 2.17-2.04(\mathrm{~m}, 3 \mathrm{H}), 1.93(\mathrm{p}, J=7.3 \mathrm{~Hz}, 2 \mathrm{H}), 1.84-1.77(\mathrm{~m}$, 1H), 1.59 (p, $J=7.3 \mathrm{~Hz}, 2 \mathrm{H}), 1.42$ (s, 9H), 1.36-1.27 (m, 2H), 1.23 (s, 12H); ${ }^{13}$ C NMR (126 MHz, $\mathbf{C D C l}_{3}$ ): $\delta 210.2,172.8,147.8,138.9$ (C-B bs), 132.7, 130.9, 128.9, 128.2, 125.8, 119.7, 84.0, 80.2, 56.2, 50.2, 41.4, 33.5, 30.3, 28.2, 26.1, 25.9, 24.85, 24.81, 23.0; ${ }^{11}$ B NMR (160 MHz, $\mathbf{C D C l}_{3}$ ): $\delta$ 29.5; HRMS (ESI): Calcd for $\mathrm{C}_{30} \mathrm{H}_{45} \mathrm{BN}_{3} \mathrm{O}_{5}[\mathrm{M}+\mathrm{H}]^{+}:$538.3447, Found: 538.3469; specific rotation: $[\alpha]_{\mathrm{D}^{20}}=-35.8\left(c 1.0, \mathrm{CHCl}_{3}\right)$ for an enantiomerically enriched sample of 99:1 e.r.

Enantiomeric purity was determined by HPLC analysis in comparison with authentic racemic material; Chiralcel OD-H column, 80:20 hexanes/i-PrOH, $0.8 \mathrm{~mL} / \mathrm{min}, 254 \mathrm{~nm}$. 

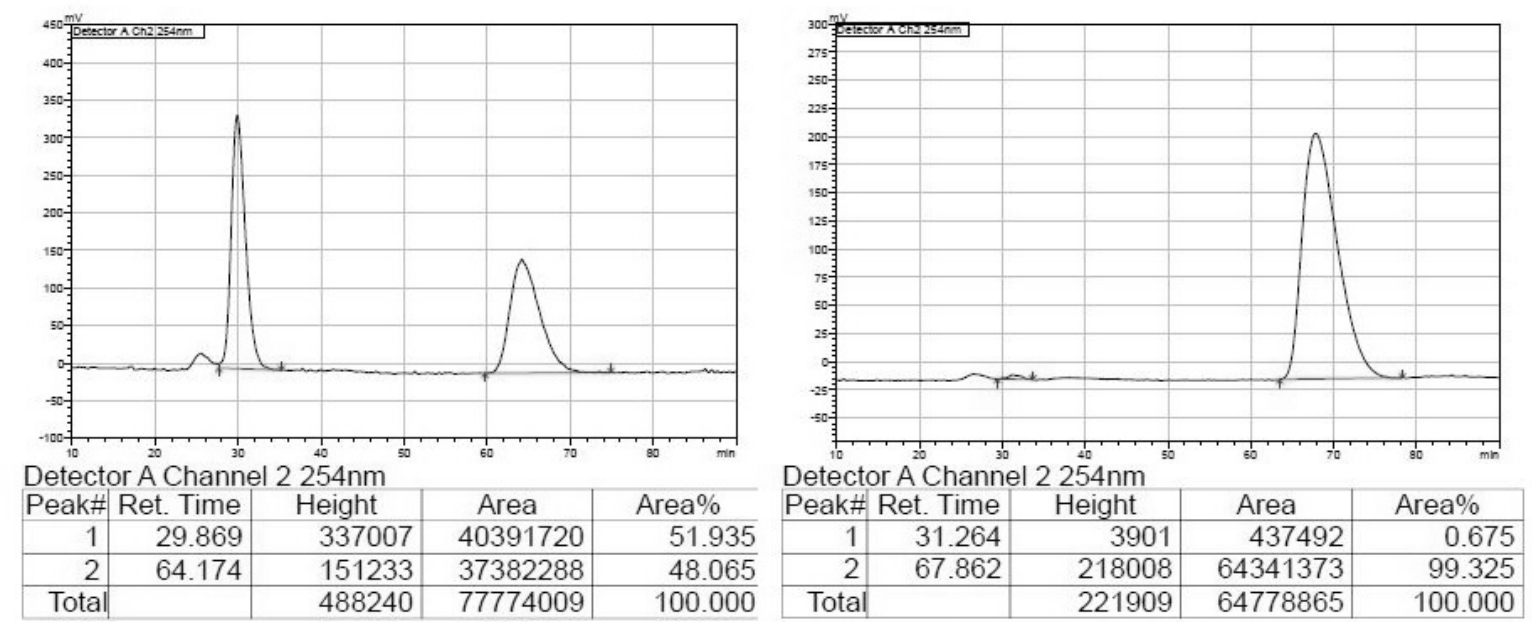

\begin{tabular}{|c|c|c|c|c|c|}
\hline Retention Time & Area & Area $\%$ & Retention Time & Area & Area\% \\
\hline 29.869 & 40391720 & 51.935 & 31.264 & 437492 & 0.675 \\
\hline 64.174 & 37382288 & 48.065 & 67.862 & 64341373 & 99.325 \\
\hline
\end{tabular}

\subsection{7 (R)-3-(2-((t-Butyldimethylsilyl)oxy)ethyl)-4-(4,4,5,5-tetramethyl-1,3,2-}

\section{dioxaborolan-2-yl)pent-4-en-2-one (4g)}

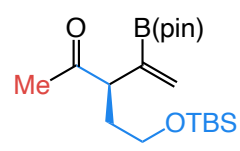

Modified procedure: $2.0 \mathrm{~mol} \% \mathrm{CuMes}(36.4 \mathrm{mg}, 0.200 \mathrm{mmol})$ and $2.2 \mathrm{~mol} \%$ phos-1 (119.2 $\mathrm{mg}, 0.220 \mathrm{mmol})$ were used.

Colorless oil; 3.31 g, 8.99 mmol, 90\% yield, 99:1 e.r.; IR (neat): 2927 (w), 2855 (w), 1712 (s), 1354 (m), 1312 (m), 1251 (m), 1138 (s), 1100 (s), 833 (s), 774 (s) cm ${ }^{-1} ;{ }^{1} \mathbf{H}$ NMR (CDCl $\mathbf{3 0 0}$ MHz): $\delta 5.96(\mathrm{~d}, J=2.7 \mathrm{~Hz}, 1 \mathrm{H}), 5.67(\mathrm{~d}, J=2.0 \mathrm{~Hz}, 1 \mathrm{H}), 3.61-3.49(\mathrm{~m}, 2 \mathrm{H}), 3.46(\mathrm{t}, J=7.1$ $\mathrm{Hz}, 1 \mathrm{H}), 2.11(\mathrm{~s}, 3 \mathrm{H}), 2.10-2.04(\mathrm{~m}, 1 \mathrm{H}), 1.75-1.67(\mathrm{~m}, 1 \mathrm{H}), 1.25(\mathrm{~s}, 12 \mathrm{H}), 0.88(\mathrm{~s}, 9 \mathrm{H}), 0.01$ (s, 6H); ${ }^{13} \mathbf{C ~ N M R ~ ( C D C l} 3$, 101 MHz): $\delta 209.3,139.4$ (C-B, bs), 132.1, 83.8, 61.0, 54.6, 33.4, 29.2, 26.1, 24.9, 24.8, 18.4, -5.19, -5.22; ${ }^{11} \mathbf{B}$ NMR (CDCl, 160 MHz): $\delta 29.8$; HRMS (DART): Calcd for $\mathrm{C}_{19} \mathrm{H}_{38} \mathrm{BO}_{4} \mathrm{Si}[\mathrm{M}+\mathrm{H}]^{+}: 369.2627$, Found: 369.2621 ; specific rotation: $[\alpha]_{\mathrm{D}^{20}}=-68.8\left(c 1.5, \mathrm{CHCl}_{3}\right)$ for an enantiomerically enriched sample of 99:1 e.r.

Enantiomeric purity was determined by HPLC analysis in comparison with authentic racemic material; Chiralcel AD-H column, 99.9:0.1 hexanes $/ i-\mathrm{PrOH}, 0.3 \mathrm{~mL} / \mathrm{min}, 220 \mathrm{~nm}$.
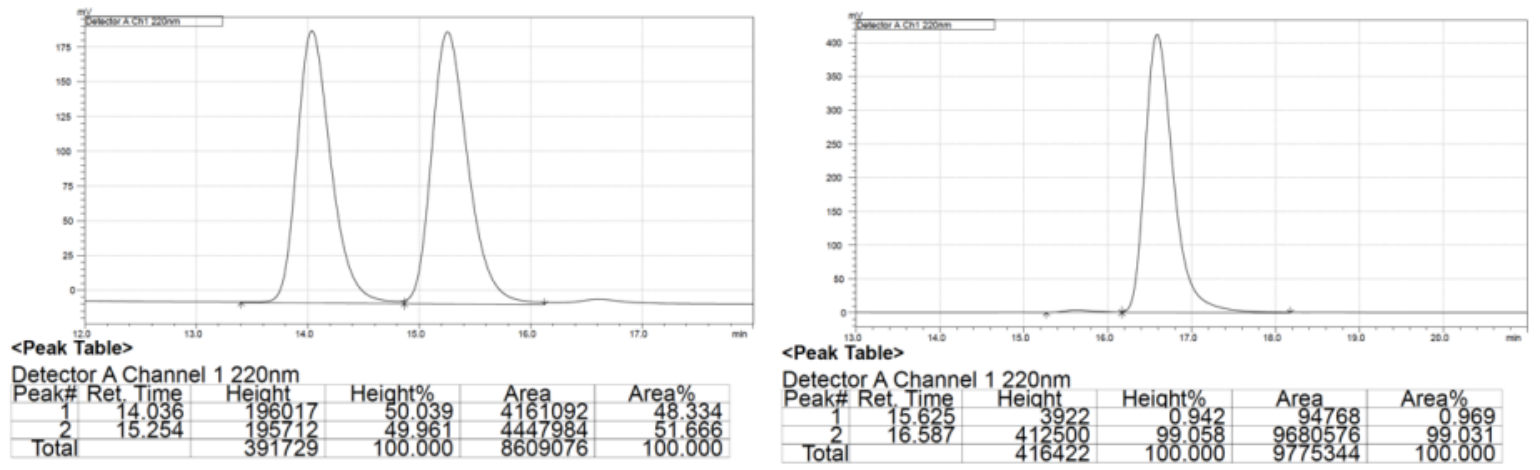


\begin{tabular}{|c|c|c|c|c|c|}
\hline Retention Time & Area & Area $\%$ & Retention Time & Area & Area\% \\
\hline 14.036 & 4161092 & 48.344 & 15.625 & 94768 & 0.969 \\
\hline 15.254 & 4447984 & 51.666 & 16.587 & 9680576 & 99.031 \\
\hline
\end{tabular}

6.4.8 t-Butyl (S)-(2-acetyl-3-(4,4,5,5-tetramethyl-1,3,2-dioxaborolan-2-yl)but-3-en-1yl)carbamate (4h)<smiles>C=C(Br)C(CNC(=O)O)C(C)=O</smiles>

Colorless oil; 23.6 mg, 0.0696 mmol, 70\% yield, 97:3 e.r.; IR (neat): 3364 (br), 2975 (m), 2929 (w), 1708 (s), 1612 (w), 1500 (m), 1429 (m), 1364 (s), 1314 (s), 1270 (m), 1249 (m), 1161 (s), 1138 (s), 966 (m), 863 (w), 683 (w) cm ${ }^{-1} ;{ }^{1}$ H NMR (500 MHz, CDCl $\left.\mathbf{~}_{3}\right): \delta 6.02$ (d, $J$ $=2.5 \mathrm{~Hz}, 1 \mathrm{H}), 5.70(\mathrm{~d}, J=2.5 \mathrm{~Hz}, 1 \mathrm{H}), 5.04(\mathrm{~s}, 1 \mathrm{H}), 3.53-3.18(\mathrm{~m}, 3 \mathrm{H}), 2.10(\mathrm{~s}, 3 \mathrm{H}), 1.40(\mathrm{~s}$, $9 \mathrm{H}), 1.26(\mathrm{~s}, 12 \mathrm{H})$. The signals are broadened due to equilibrium of carbamate rotamers; ${ }^{13} \mathrm{C}$ NMR (126 MHz, $\mathbf{C D C l}_{3}$ ): $\delta$ 208.8, 156.0, 133.7, 84.2, 79.1, 58.4, 41.2, 29.1, 28.5, 24.9, 24.8; The carbon bearing the boron atom could not be detected due to quadrupolar effects; ${ }^{11} \mathbf{B}$ NMR (160 MHz, $\mathbf{C D C l}_{3}$ ): $\delta$ 30.6; HRMS (DART): Calcd for $\mathrm{C}_{17} \mathrm{H}_{31} \mathrm{BNO}_{5}[\mathrm{M}+\mathrm{H}]^{+}: 340.2290$, Found: 340.2286 ; specific rotation: $[\alpha]_{\mathrm{D}}{ }^{20}=-71.1\left(c\right.$ 1.0, $\left.\mathrm{CHCl}_{3}\right)$ for an enantiomerically enriched sample of 97:3 e.r.

Enantiomeric purity was determined by HPLC analysis in comparison with authentic racemic material; Chiralcel OZ-H column, 98:2 hexanes/ $i-\mathrm{PrOH}, 0.6 \mathrm{~mL} / \mathrm{min}, 220 \mathrm{~nm}$.
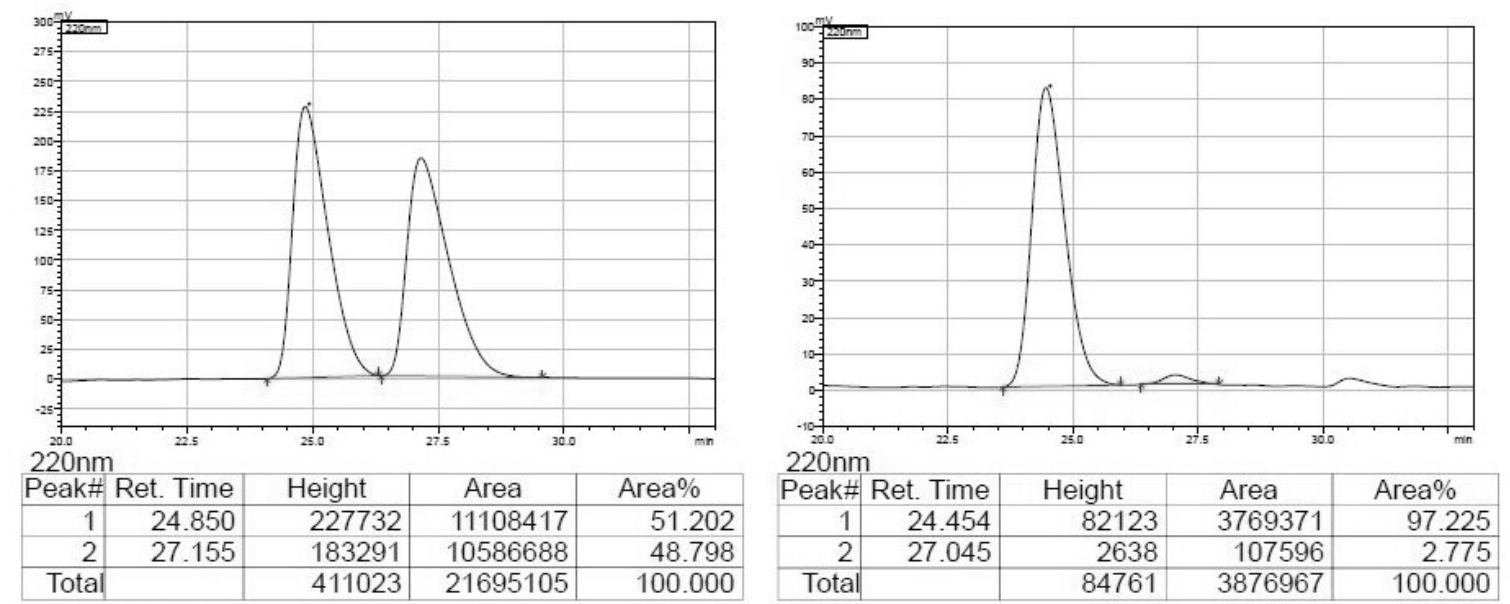

\begin{tabular}{|c|c|c|c|c|c|}
\hline Retention Time & Area & Area\% & Retention Time & Area & Area\% \\
\hline 24.850 & 11108417 & 51.202 & 24.454 & 3769371 & 97.225 \\
\hline 27.155 & 10586688 & 48.798 & 27.045 & 107569 & 2.775 \\
\hline
\end{tabular}

6.4.9 (S)-3-Phenyl-4-(4,4,5,5-tetramethyl-1,3,2-dioxaborolan-2-yl)pent-4-en-2-one (4i)<smiles>C=C(Br)C(Br)c1ccccc1</smiles> 
Modified procedure: The mixture was allowed to warm to $22{ }^{\circ} \mathrm{C}$, and kept as such for 5 min before the reaction was quenched by addition of an aqueous saturated solution of $\mathrm{NH}_{4} \mathrm{Cl}$.

Colorless oil; $22.6 \mathrm{mg}, 0.079$ mmol, 79\% yield, 96:4 e.r.; IR (neat): 2975 (m), 2922 (m), 2852 (w), 1712 (m), 1361 (s), 1310 (m), 1133 (s), 703 (w) cm ${ }^{-1} ;{ }^{1} \mathbf{H}$ NMR (600 MHz, CDCl $)$ : $\delta$ 7.36-7.30 (m, 2H), 7.29-7.24 (m, 1H), 7.18-7.14 (m, 2H), $5.93(\mathrm{~s}, 1 \mathrm{H}), 5.20(\mathrm{~s}, 1 \mathrm{H}), 4.65(\mathrm{~s}$, 1H), 2.12 (s, 3H), 1.24 (s, 12H); ${ }^{13} \mathbf{C}$ NMR (192 $\left.\mathbf{~ M H z , ~} \mathbf{C D C l}_{3}\right): \delta 207.7,140.5$ (C-B bs), 136.8, $130.5,129.9,128.8,127.3,83.9,63.9,29.9,29.6,24.9,24.7$. The ortho protons in the aromatic ring are inequivalent due to slow rotation of the phenyl ring; ${ }^{11} \mathbf{B}$ NMR (192 $\left.\mathbf{M H z}, \mathbf{C D C l}_{3}\right)$ : $\delta$ 29.6; HRMS (DART): Calcd for $\mathrm{C}_{17} \mathrm{H}_{24} \mathrm{BO}_{3}[\mathrm{M}+\mathrm{H}]^{+}$: 287.18130, Found: 287.18215; specific rotation: $[\alpha]_{\mathrm{D}}{ }^{20}=+119.2\left(c 1.0, \mathrm{CHCl}_{3}\right)$ for an enantiomerically enriched sample of $96: 4$ e.r. Enantiomeric purity was determined by HPLC analysis in comparison with authentic racemic material; Chiralcel OD-H column, 99.5:0.5 hexanes $/ i-\mathrm{PrOH}, 0.5 \mathrm{~mL} / \mathrm{min}, 210 \mathrm{~nm}$.
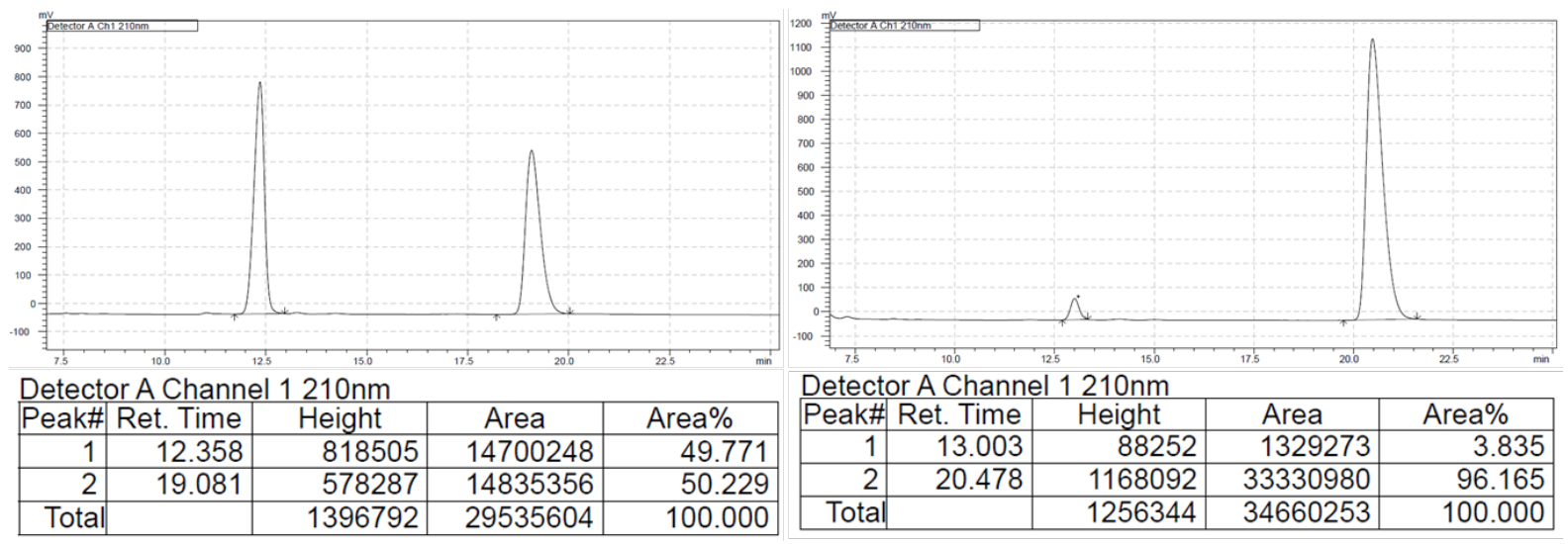

\begin{tabular}{|c|c|c|c|c|c|}
\hline Retention Time & Area & Area\% & Retention Time & Area & Area\% \\
\hline 12.358 & 14700248 & 49.771 & 13.003 & 1329273 & 3.835 \\
\hline 19.081 & 14835356 & 50.229 & 20.478 & 33330980 & 96.165 \\
\hline
\end{tabular}

6.4.10 $(R)$-3-(2-Fluoroacetyl)-4-(4,4,5,5-tetramethyl-1,3,2-dioxaborolan-2-yl)pent-4-en1-yl (t-butoxycarbonyl)-L-valinate $(4 \mathrm{j})$<smiles>C=C(O)C(CCOC(=O)C(CC)NC(=O)O)C(=O)O</smiles>

Modified procedure: $38.0 \mathrm{mg}$ of $\mathrm{B}_{2}$ (pin) $)_{2}(0.150 \mathrm{mmol}, 1.5$ equivalents $)$ and $3.8 \mathrm{mg}$ of $\mathrm{MeOH}$ ( 0.120 mmol, 1.2 equivalents). The reaction was performed for $8 \mathrm{~h}$ at $-40{ }^{\circ} \mathrm{C}$ to minimize epimerization.

Yellow oil; 22.0 mg, 0.0811 mmol, 81\% yield, 98:2 d.r., >99:1 e.r.; IR (neat): 3378 (br), 2972 (m), 2930 (w), 2873 (w), 1735 (s), 1714 (s), 1499 (m), 1428 (m), 1428 (m), 1364 (s), 1314 (s), 1245 (m), 1156 (s), 1139 (s), 1089 (m), 1044 (m), 1018 (m), 967 (m), 863 (w) cm ${ }^{-1},{ }^{1} \mathbf{H}$ NMR (600 MHz, toluene-ds): $\delta 6.06(\mathrm{~d}, J=2.5 \mathrm{~Hz}, 1 \mathrm{H}), 5.54(\mathrm{~d}, J=2.5 \mathrm{~Hz}, 1 \mathrm{H}), 5.07(\mathrm{~d}, J=9.0$ $\mathrm{Hz}, 1 \mathrm{H}), 4.55-4.32(\mathrm{~m}, 3 \mathrm{H}), 4.08-3.99(\mathrm{~m}, 1 \mathrm{H}), 3.95-3.86(\mathrm{~m}, 1 \mathrm{H}), 3.54-3.51(\mathrm{~m}, 1 \mathrm{H}), 2.18-$ $2.09(\mathrm{~m}, 1 \mathrm{H}), 2.07-1.99(\mathrm{~m}, 1 \mathrm{H}), 1.86-1.77(\mathrm{~m}, 1 \mathrm{H}), 1.41(\mathrm{~s}, 9 \mathrm{H}), 1.03(\mathrm{~s}, 12 \mathrm{H}), 0.89(\mathrm{~d}, J=$ 
$6.9 \mathrm{~Hz}, 3 \mathrm{H}), 0.75(\mathrm{~d}, J=6.9 \mathrm{~Hz}, 3 \mathrm{H}) ;{ }^{13} \mathrm{C}$ NMR (151 MHz, toluene-d $)$ ): $\delta 204.2(\mathrm{~d}, J=17.9$ Hz), 172.2, 155.7, 133.0, 84.6 (d, $J=185.9$ Hz), 84.0, 79.2, 63.0, 58.9, 49.0, 31.6, 29.9, 28.4, $24.63,24.62,19.2,17.6$. The carbon bearing the boron atom could not be detected due to quadrupolar effects; ${ }^{11} \mathrm{~B}$ NMR (192 MHz, toluene- $\left.\boldsymbol{d}_{8}\right)$ : $\delta 30.3 ;{ }^{19} \mathrm{~F}$ NMR (564 MHz, toluened8): $\delta-227.81$ ( $\mathrm{t}, J=47.9 \mathrm{~Hz}$, major diastereomer), $-227.84(\mathrm{t}, J=45.1 \mathrm{~Hz}$, minor diastereomer) The two sets of signals for the major diastereomer correspond to an equilibrium between carbamate rotamers; HRMS (DART): Calcd for $\mathrm{C}_{23} \mathrm{H}_{40} \mathrm{BNO}_{7} \mathrm{~F}[\mathrm{M}+\mathrm{H}]^{+}$: 472.2876, Found: 472.2886; specific rotation: $[\alpha]_{\mathrm{D}}{ }^{20}=-74.4\left(c 1.0, \mathrm{CHCl}_{3}\right)$ for an enantiomerically enriched sample of $>99: 1$ e.r. (corresponding to the enantiomeric purity of the allene, see section 4.1.6.

6.4.11 (S)-3-(2-Fluoroacetyl)-4-(4,4,5,5-tetramethyl-1,3,2-dioxaborolan-2-yl)pent-4-en1-yl (t-butoxycarbonyl)-L-valinate (4k)

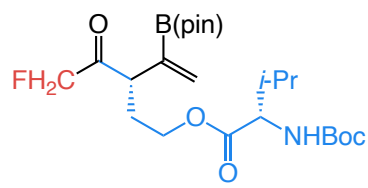

The general procedure was modified: $38.0 \mathrm{mg}$ of $\mathrm{B}_{2}(\text { pin })_{2}(0.150 \mathrm{mmol}, 1.5$ equivalents $)$ and $3.8 \mathrm{mg}$ of $\mathrm{MeOH}(0.120 \mathrm{mmol}, 1.2$ equivalents $)$. The reaction was carried out for $8 \mathrm{~h}$ at $-40{ }^{\circ} \mathrm{C}$ to minimize epimerization.

Yellow oil; 21.9 mg, 0.0807 mmol, 81\% yield, 98:2 d.r., >99:1 e.r.; IR (neat): 3379 (br), 2972 (m), 2931 (w), 1713 (s), 1499 (m), 1428 (m), 1364 (s), 1318 (s), 1245 (m), 1156 (s), 1138 (s), $1018(\mathrm{~m}), 863(\mathrm{~m}), 672(\mathrm{w}) \mathrm{cm}^{-1} ;{ }^{1} \mathbf{H}$ NMR (600 MHz, toluene-d8): $\delta 6.06(\mathrm{~d}, J=2.6 \mathrm{~Hz}, 1 \mathrm{H})$, $5.55(\mathrm{~d}, J=2.6 \mathrm{~Hz}, 1 \mathrm{H}), 5.01(\mathrm{br}, 1 \mathrm{H}), 4.57-4.31(\mathrm{~m}, 3 \mathrm{H}), 4.07-4.00(\mathrm{~m}, 1 \mathrm{H}), 3.96-3.88(\mathrm{~m}$, $1 \mathrm{H}), 3.56-3.49(\mathrm{~m}, 1 \mathrm{H}), 2.19-2.11(\mathrm{~m}, 1 \mathrm{H}), 2.07-1.97(\mathrm{~m}, 1 \mathrm{H}), 1.87-1.79(\mathrm{~m}, 1 \mathrm{H}), 1.41(\mathrm{~s}$, 9H), $1.03(\mathrm{~s}, 12 \mathrm{H}), 0.88(\mathrm{~d}, J=6.9 \mathrm{~Hz}, 3 \mathrm{H}), 0.74(\mathrm{~d}, J=6.9 \mathrm{~Hz}, 3 \mathrm{H}) ;{ }^{13} \mathbf{C}$ NMR $(151 \mathrm{MHz}$, toluene- $\left.d_{8}\right): \delta 204.1(\mathrm{~d}, J=17.5 \mathrm{~Hz}), 172.1,155.7,133.0(\mathrm{~d}, J=7.0 \mathrm{~Hz}), 84.6(\mathrm{~d}, J=185.8$ $\mathrm{Hz}$ ), 84.0, 79.2, 63.0, 59.0, 49.2, 31.6, 29.9, 28.4, 24.6, 19.2, 17.7; the carbon bearing the boron atom could not be detected due to quadrupolar effects; ${ }^{11}$ B NMR (160 MHz, toluene-d $)$ : $\delta$ 30.2; ${ }^{19}$ F NMR (564 MHz, toluene- $\left.d_{8}\right): \delta-227.84$ (t, $J=45.1 \mathrm{~Hz}$, major diastereomer), 227.81 ( $\mathrm{t}, J=47.9 \mathrm{~Hz}$, major diastereomer), -227.81 ( $\mathrm{t}, J=47.7 \mathrm{~Hz}$, major diastereomer). The two sets of signals for the minor diastereomer are due to an equilibrium between carbamate rotamers; HRMS (ESI): Calcd for $\mathrm{C}_{23} \mathrm{H}_{40} \mathrm{BNO}_{7} \mathrm{~F}[\mathrm{M}+\mathrm{H}]^{+}$: 472.2876, Found: 472.2887; specific rotation: $[\alpha]_{\mathrm{D}}{ }^{20}=+61.8\left(c 1.0, \mathrm{CHCl}_{3}\right)$ an enantiomerically enriched sample of $>99: 1$ e.r (corresponding to the enantiomeric purity of the allene, see Section 4.1.6.

6.4.12 t-Butyl (S)-(4,4-dimethyl-3-oxo-2-(1-(4,4,5,5-tetramethyl-1,3,2-dioxaborolan-2yl)vinyl)pentyl)carbamate (4I)

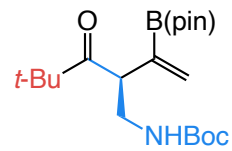

Modified procedure: $t$-Butyl-buta-2,3-dien-1-ylcarbamate $(16.9 \mathrm{mg}, 0.100 \mathrm{mmol})$ and $t$ butylnitrile (24.9 mg, $0.300 \mathrm{mmol}$ ) were used, and the reaction was carried out for $16 \mathrm{~h}$ at $26{ }^{\circ} \mathrm{C}$. 
Colorless oil; $33.2 \mathrm{mg}, 0.0871 \mathrm{mmol}$, 87\% yield, >99:1 e.r.; IR (neat): 3387 (br), 2974 (m), 2929 (m), 2869 (w), 1715 (s), 1698 (s), 1499 (m), 1478 (m), 1365 (m), 1314 (m), 1250 (m), 1168 (s), 1138 (s), 952 (w), 869 (w), $851(\mathrm{w}) \mathrm{cm}^{-1}$; NMR data at $80{ }^{\circ} \mathrm{C}:{ }^{\mathbf{1}} \mathbf{H}$ NMR (600 MHz, toluene-ds): $\delta 5.99(\mathrm{~s}, 1 \mathrm{H}), 5.68(\mathrm{~s}, 1 \mathrm{H}), 4.76(\mathrm{br}, 1 \mathrm{H}), 4.20(\mathrm{t}, J=6.5 \mathrm{~Hz}, 1 \mathrm{H}), 3.50-3.29(\mathrm{~m}$, $2 \mathrm{H}), 1.41(\mathrm{~d}, J=1.9 \mathrm{~Hz}, 9 \mathrm{H}), 1.13(\mathrm{~d}, J=11.1 \mathrm{~Hz}, 12 \mathrm{H}), 1.07(\mathrm{~s}, 9 \mathrm{H})$; NMR data at $25^{\circ} \mathrm{C}$ : ${ }^{1}$ H NMR (600 MHz, toluene-d8): $\delta 6.04(\mathrm{t}, J=2.4 \mathrm{~Hz}, 1 \mathrm{H}), 5.68(\mathrm{~d}, J=2.9 \mathrm{~Hz}, 1 \mathrm{H}), 4.89$ (br, $1 \mathrm{H}), 4.36-4.17(\mathrm{~m}, 1 \mathrm{H}), 3.48-3.27(\mathrm{~m}, 2 \mathrm{H}), 1.38(\mathrm{~s}, 9 \mathrm{H}), 1.08(\mathrm{~s}, 12 \mathrm{H}), 1.03(\mathrm{~s}, 9 \mathrm{H})$; two sets of signals are due to carbamate rotamers; ${ }^{13} \mathbf{C}$ NMR (151 MHz, toluene-ds): $\delta$ 214.6, 155.8, $131.8,83.9,78.2,50.1,44.5,43.8,28.5,24.7$. The carbon bearing the boron atom could not be detected due to quadrupolar effects; ${ }^{11}$ B NMR (160 MHz, toluene-d8) $\delta 31.1$; HRMS (DART): Calcd for $\mathrm{C}_{20} \mathrm{H}_{37} \mathrm{BNO}_{5}[\mathrm{M}+\mathrm{H}]^{+}: 382.2759$, Found: 382.2777 ; specific rotation: $[\alpha]_{\mathrm{D}}{ }^{20}=-37.4\left(c 1.0, \mathrm{CHCl}_{3}\right)$ for an enantiomerically enriched sample of $>99: 1$ e.r.

Enantiomeric purity was determined by HPLC analysis in comparison with authentic racemic material; Chiralcel OZ-H column, 95:5 hexanes/i-PrOH, $0.5 \mathrm{~mL} / \mathrm{min}, 220 \mathrm{~nm}$.
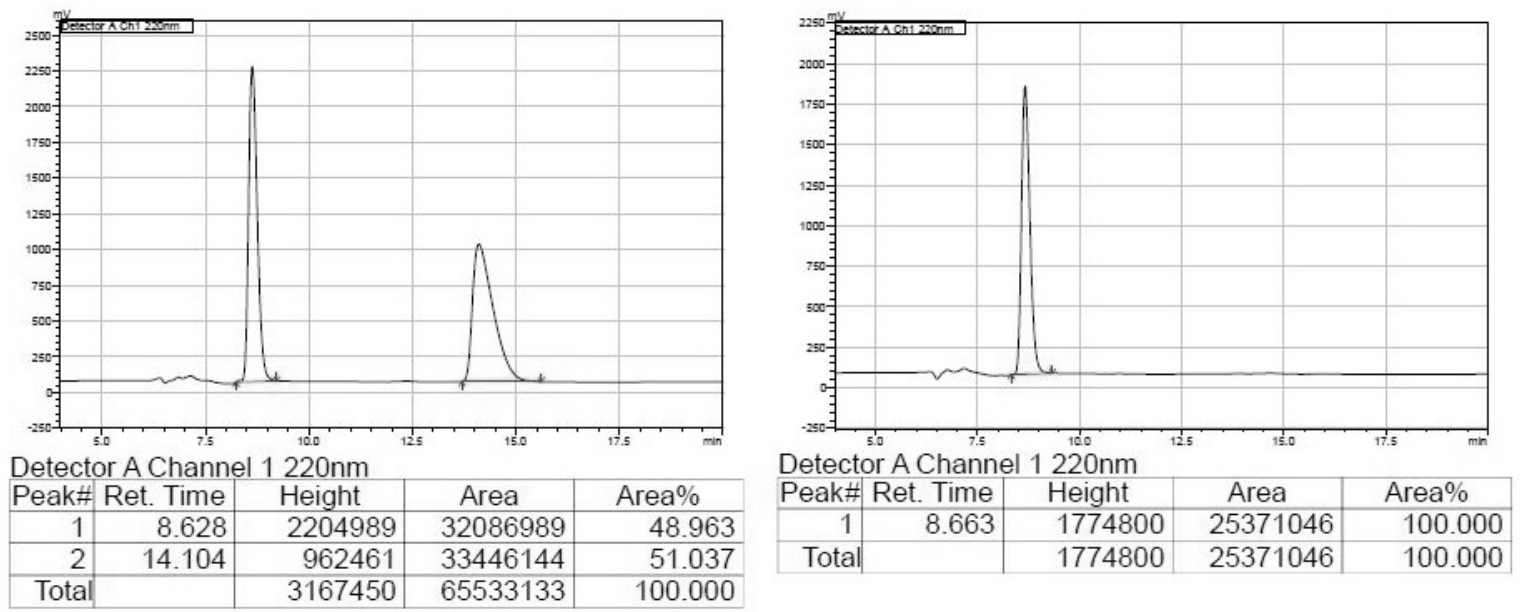

\begin{tabular}{|c|c|c|c|c|c|}
\hline Retention Time & Area & Area\% & Retention Time & Area & Area $\%$ \\
\hline 8.628 & 32086989 & 48.963 & 8.663 & 25371046 & 100.000 \\
\hline 14.104 & 33446144 & 51.037 & & & \\
\hline
\end{tabular}

6.4.13 (R)-3-(2-((t-Butyldimethylsilyl)oxy)ethyl)-2-(4,4,5,5-tetramethyl-1,3,2dioxaborolan-2-yl)hepta-1,6-dien-4-one (4m)

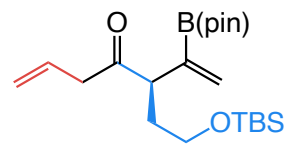

Modified procedure: $2.0 \mathrm{~mol} \%$ CuMes (18.2 mg, $0.200 \mathrm{mmol})$ and $2.2 \mathrm{~mol} \%$ phos-1 (59.6 $\mathrm{mg}, 0.110 \mathrm{mmol}$ ) was used.

Colorless oil; 1.66 g, 4.20 mmol, 84\% yield, 97:3 e.r.; IR (neat): 2926 (w), 2854 (w), 1712 (m), 1312 (m), 1251 (m), 1139 (s), 1096 (m), 833 (s), 774 (s) cm ${ }^{-1} ;{ }^{1} \mathbf{H}$ NMR (CDCl, 500 MHz): $\delta 5.96(\mathrm{~d}, J=2.7 \mathrm{~Hz}, 1 \mathrm{H}), 5.90(\mathrm{ddt}, J=17.2,10.2,6.9 \mathrm{~Hz}, 1 \mathrm{H}), 5.66(\mathrm{~d}, J=3.2 \mathrm{~Hz}$, 1H), 5.15-5.02 (m, 2H), 3.60-3.49 (m, 3H), 3.20 (ddt, $J=7.2,4.5,1.4 \mathrm{~Hz}, 2 \mathrm{H}), 2.17-1.95$ (m, 
1H), 1.76-1.65 (m, 1H), $1.25(\mathrm{~s}, 12 \mathrm{H}), 0.87(\mathrm{~s}, 9 \mathrm{H}), 0.01(\mathrm{~s}, 3 \mathrm{H}), 0.01(\mathrm{~s}, 3 \mathrm{H}) ;{ }^{13} \mathbf{C ~ M R}\left(\mathbf{C D C l}_{\mathbf{3}}\right.$, $101 \mathrm{MHz}): \delta 208.8,132.3,131.3,118.3,83.9,61.0,53.1,46.7,33.6,26.1,24.88,24.85,18.4$, $-5.18,-5.20$; ${ }^{11} \mathbf{B}$ NMR (CDCl, 160 MHz): $\delta 29.8$; HRMS (DART): Calcd for $\mathrm{C}_{19} \mathrm{H}_{38} \mathrm{BO}_{4} \mathrm{Si}$ $[\mathrm{M}+\mathrm{H}]^{+}:$395.2783, Found: 395.2778 ; specific rotation: $[\alpha]_{\mathrm{D}}{ }^{20}=-59.2\left(c 1.5, \mathrm{CHCl}_{3}\right)$ for an enantiomerically enriched sample of 97:3 e.r.

Enantiomeric purity was determined by HPLC analysis in comparison with authentic racemic material; Chiralcel AD-H column, 99.9:0.1 hexanes/i-PrOH, 0.3 mL/min, $220 \mathrm{~nm}$.
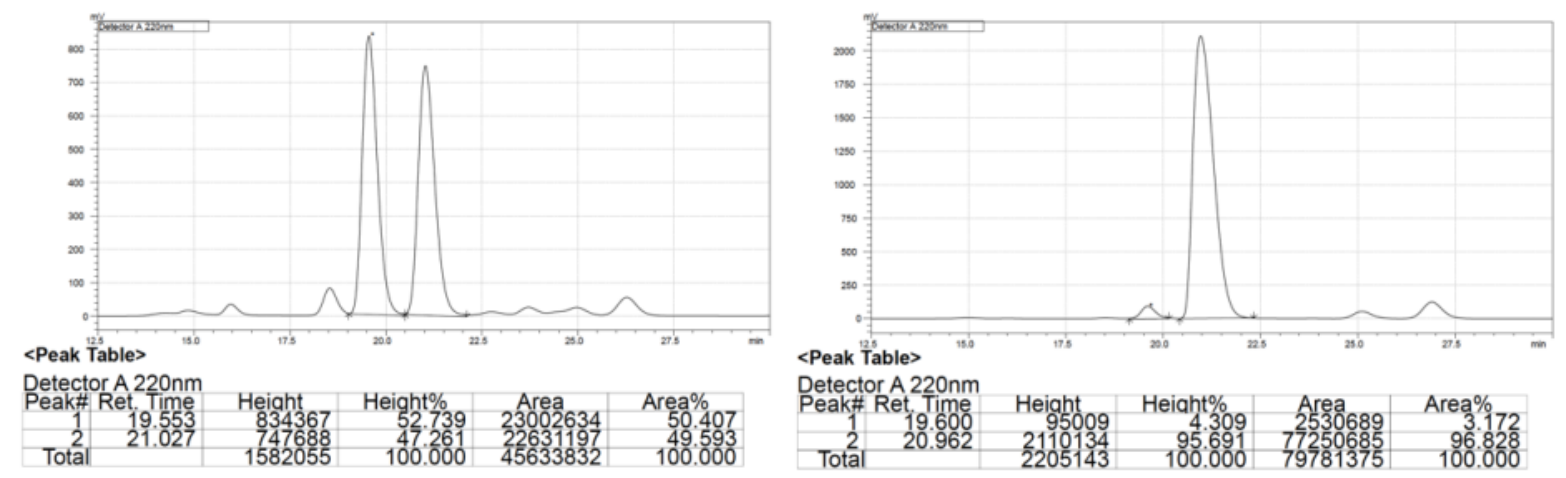

\begin{tabular}{|c|c|c|c|c|c|}
\hline Retention Time & Area & Area\% & Retention Time & Area & Area \% \\
\hline 19.553 & 23002634 & 50.407 & 19.600 & 2530689 & 3.172 \\
\hline 21.027 & 22631197 & 49.593 & 20.962 & 77250685 & 96.828 \\
\hline
\end{tabular}

6.4.14 (R)-3-(2-((t-Butyldimethylsilyl)oxy)ethyl)-2-(4,4,5,5-tetramethyl-1,3,2dioxaborolan-2-yl)-7-(trimethylsilyl)hept-1-en-6-yn-4-one (4n)

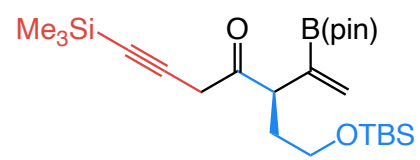

Colorless oil; $30.2 \mathrm{mg}, 0.065 \mathrm{mmol}, 65 \%$ yield, 97:3 e.r.; IR (neat): 2975 (m), 2922 (m), 2852 (w), 1712 (m), 1361 (s), 1310 (m), 1133 (s), 703 (w) cm ${ }^{-1}$; ${ }^{\mathbf{1}} \mathbf{H}$ NMR (500 MHz, CDCl $)$ : $\delta$ $5.99(\mathrm{~d}, J=2.6 \mathrm{~Hz}, 1 \mathrm{H}), 5.72(\mathrm{~d}, J=2.6 \mathrm{~Hz}, 1 \mathrm{H}), 3.68(\mathrm{t}, J=7.0 \mathrm{~Hz}, 1 \mathrm{H}), 3.61-3.50(\mathrm{~m}, 2 \mathrm{H})$, 3.37 (s, 2H), 2.16-2.05 (m, 1H), 1.78-1.65 (m, 1H), $1.25(\mathrm{~s}, 12 \mathrm{H}), 0.88(\mathrm{~s}, 9 \mathrm{H}), 0.16(\mathrm{~s}, 9 \mathrm{H})$, 0.02 (s, 6H); ${ }^{13} \mathbf{C}$ NMR (126 MHz, $\mathbf{C D C l}_{3}$ ): $\delta$ 204.0, 133.0, 99.1, 89.1, 84.0, 60.9, 52.7, 34.4, $33.6,29.9,26.1,24.9,24.8,18.4,0.1,-5.2$; the carbon bearing the boron atom could not be detected due to quadrupolar effects; ${ }^{11} \mathbf{B}$ NMR (160 $\left.\mathbf{M H z}, \mathbf{C D C l}_{3}\right): \delta 29.9$; HRMS (DART): Calcd for $\mathrm{C}_{17} \mathrm{H}_{24} \mathrm{BO}_{3}[\mathrm{M}+\mathrm{H}]^{+}: 287.1813$, Found: 287.1822 ; specific rotation: $[\alpha]_{\mathrm{D}}{ }^{20}=-42.8$ $\left(c 1.0, \mathrm{CHCl}_{3}\right)$ for an enantiomerically enriched sample of $97: 3$ e.r.

Enantiomeric purity was determined by HPLC analysis in comparison with authentic racemic material; $(R, R)$-Whelk-O1 column, 100:0 hexanes $/ i-\mathrm{PrOH}, 0.5 \mathrm{~mL} / \mathrm{min}, 220 \mathrm{~nm}$. As a result of a slight signal overlap, there is greater uncertainty in the determination of this enantiomeric ratio, which we estimate it to be 97.3 e.r. 

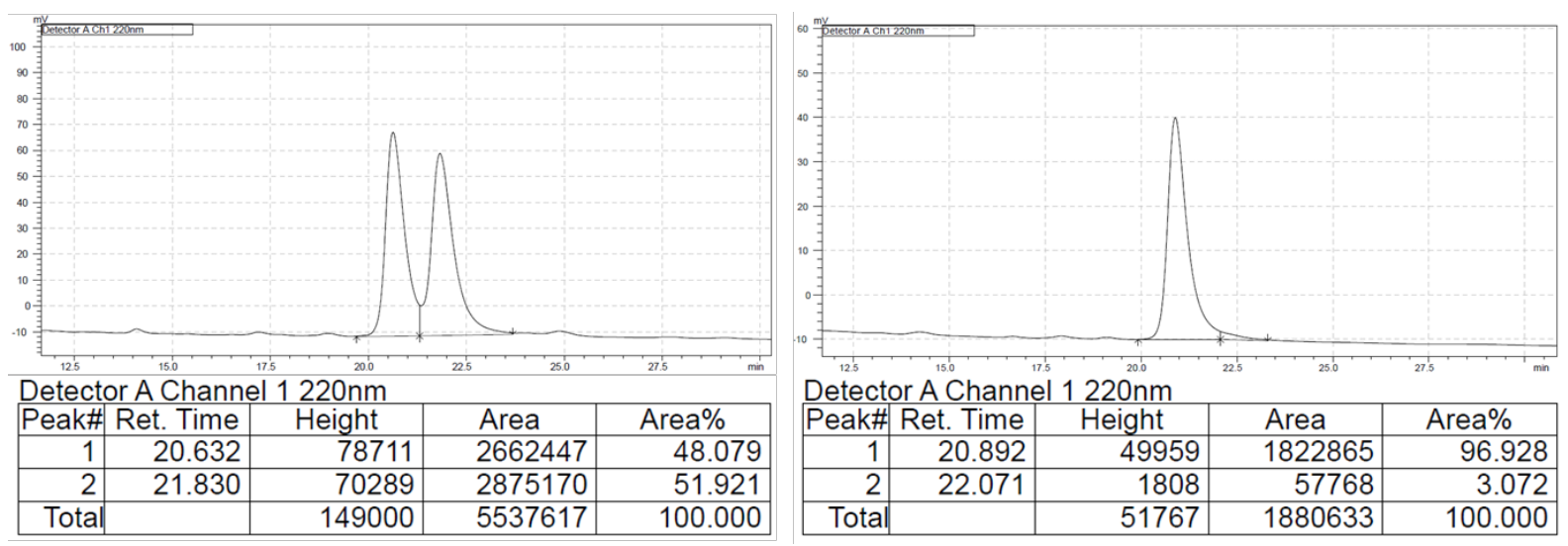

\begin{tabular}{|c|c|c|c|c|c|}
\hline Retention Time & Area & Area\% & Retention Time & Area & Area $\%$ \\
\hline 20.632 & 2662447 & 48.079 & 20.892 & 1822865 & 96.928 \\
\hline 21.830 & 2875170 & 51.921 & 22.071 & 57768 & 3.072 \\
\hline
\end{tabular}

6.4.15 $t$-Butyl (R)-4-(2-((t-butyldimethylsilyl)oxy)ethyl)-3-oxo-5-(4,4,5,5-tetramethyl1,3,2-dioxaborolan-2-yl)hex-5-enoate (4o)

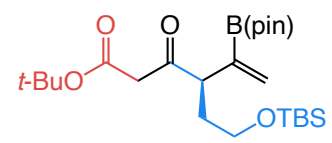

Modified procedure: $2.0 \mathrm{~mol} \%$ CuMes (18.2 mg, $0.200 \mathrm{mmol})$ and $2.2 \mathrm{~mol} \%$ phos-1 $(59.6$ $\mathrm{mg}, 0.110 \mathrm{mmol}$ ) was used and the reaction was carried for $36 \mathrm{~h}$ at $-40{ }^{\circ} \mathrm{C}$.

Colorless oil; 1.31 g, 2.80 mmol, 56\% yield, 96:4 e.r.; IR (neat): 2927 (w), 2855 (w), 1739 (s), 1712 (s), 1367 (m), 1313 (m), 1253 (m), 1140 (s), 1099 (m), 834 (s), 775 (m) cm ${ }^{-1}$; ${ }^{1}$ H NMR $\left(\mathbf{C D C l}_{3}, \mathbf{5 0 0} \mathrm{MHz}\right): \delta 6.00(\mathrm{~d}, J=2.6 \mathrm{~Hz}, 1 \mathrm{H}), 5.69(\mathrm{~d}, J=2.1 \mathrm{~Hz}, 1 \mathrm{H}), 3.60-3.48(\mathrm{~m}, 4 \mathrm{H})$, $3.43-3.30(\mathrm{~m}, 2 \mathrm{H}), 2.13-2.03(\mathrm{~m}, 2 \mathrm{H}), 1.77-1.66(\mathrm{~m}, 1 \mathrm{H}), 1.43(\mathrm{~s}, 9 \mathrm{H}), 1.24(\mathrm{~s}, 12 \mathrm{H}), 0.87$ (s, 9H), 0.01 (s, 6H); ${ }^{13} \mathbf{C ~ N M R ~ ( C D C l} 3,126$ MHz): $\delta 203.6,166.8,139.0$ (C-B, bs), 133.1, 84.0,

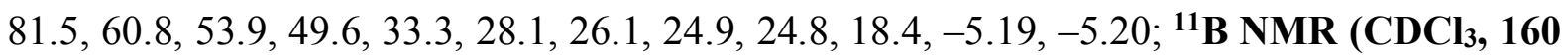
MHz): $\delta 29.8$; HRMS (DART): Calcd for $\mathrm{C}_{24} \mathrm{H}_{46} \mathrm{BO}_{6} \mathrm{Si}[\mathrm{M}+\mathrm{H}]^{+}:$469.3151, Found: 469.3148; specific rotation: $[\alpha]_{\mathrm{D}}{ }^{20}=-52.1\left(c 0.81, \mathrm{CHCl}_{3}\right)$ for an enantiomerically enriched sample of 96:4 e.r.

Enantiomeric purity was determined by HPLC analysis in comparison with authentic racemic material; Chiralcel AD-H column, 99.9:0.1 hexanes $/ i$-PrOH, 0.3 mL/min, $220 \mathrm{~nm}$.
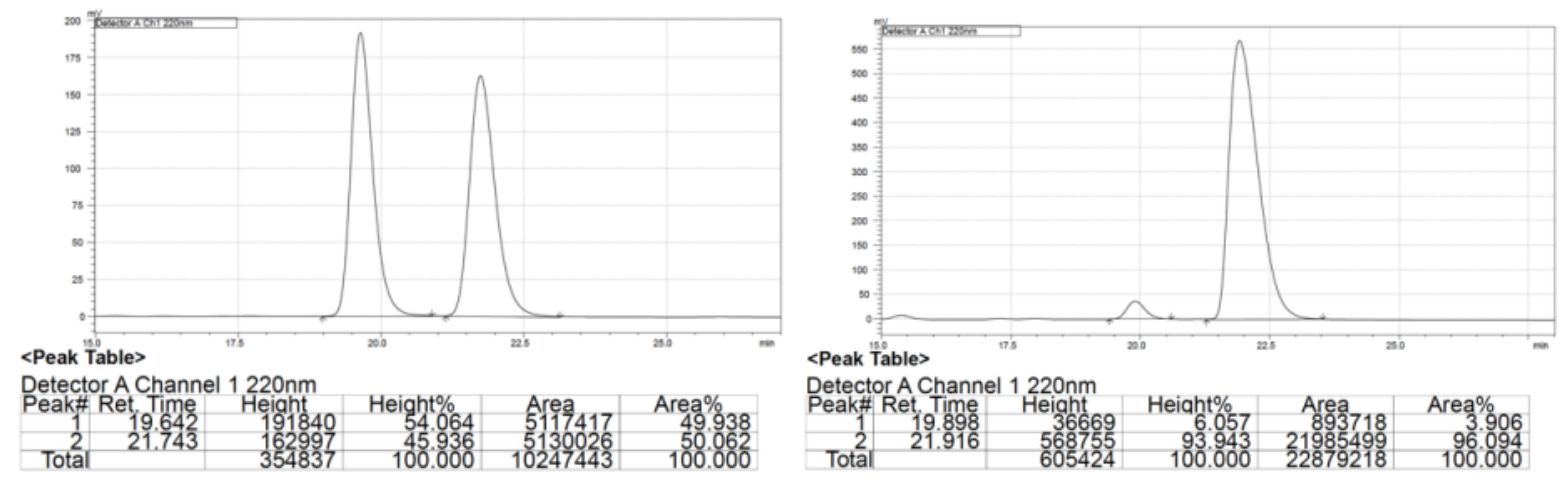


\begin{tabular}{|c|c|c|c|c|c|}
\hline Retention Time & Area & Area\% & Retention Time & Area & Area\% \\
\hline 19.642 & 5117417 & 49.938 & 19.898 & 893718 & 3.906 \\
\hline 21.743 & 5130026 & 50.062 & 21.916 & 21985499 & 96.094 \\
\hline
\end{tabular}

6.4.16 $(2 R, 4 R)-4-(2-((t$-Butyldimethylsilyl)oxy)ethyl)-2-phenyl-5-(4,4,5,5-tetramethyl1,3,2-dioxaborolan-2-yl)hex-5-en-3-one (4p)

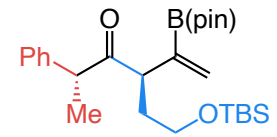

Modified procedure: $2.0 \mathrm{~mol} \%$ CuMes $(3.6 \mathrm{mg}, 0.040 \mathrm{mmol})$ and $2.2 \mathrm{~mol} \%$ phos-1 (11.9 mg, $0.110 \mathrm{mmol})$.

Colorless oil; 422 mg, 0.92 mmol, 92\% yield, 98:2 d.r., >99:1 e.r.; IR (neat): 2926 (w), 1710 (m), 1312 (m), 1139 (m), 1097 (m), 813 (s), 774 (m), 698 (m) cm ${ }^{-1} ;{ }^{1} \mathbf{H}$ NMR (CDCl, 400 MHz): $\delta 7.32-7.25(\mathrm{~m}, 2 \mathrm{H}), 7.25-7.16(\mathrm{~m}, 3 \mathrm{H}), 6.00(\mathrm{~d}, J=2.6 \mathrm{~Hz}, 1 \mathrm{H}), 5.65(\mathrm{~d}, J=2.6 \mathrm{~Hz}$, $1 \mathrm{H}), 3.89$ (q, $J=6.9 \mathrm{~Hz}, 1 \mathrm{H}), 3.54(\mathrm{t}, J=7.0 \mathrm{~Hz}, 1 \mathrm{H}), 3.35-3.17(\mathrm{~m}, 2 \mathrm{H}), 2.01-1.87(\mathrm{~m}, 1 \mathrm{H})$, $1.65-1.52(\mathrm{~m}, 1 \mathrm{H}), 1.32(\mathrm{~d}, J=6.9 \mathrm{~Hz}, 3 \mathrm{H}), 1.27(\mathrm{~s}, 12 \mathrm{H}), 0.77(\mathrm{~s}, 9 \mathrm{H}),-0.10(\mathrm{~s}, 3 \mathrm{H}),-0.14$ (s, 3H). ${ }^{13} \mathbf{C}$ NMR (CDCl 3 , 101 MHz): $\delta$ 210.2, 140.8, 139.5 (C-B, bs), 132.9, 128.9, 128.2,

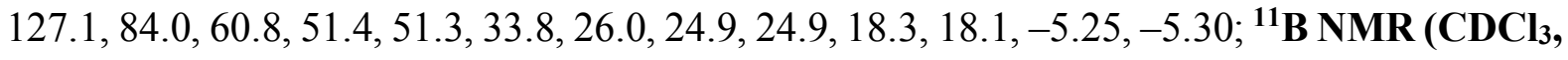
$160 \mathrm{MHz}$ ): $\delta$ 29.8; HRMS (DART): Calcd for $\mathrm{C}_{26} \mathrm{H}_{44} \mathrm{BO}_{4} \mathrm{Si}[\mathrm{M}+\mathrm{H}]^{+}$: 459.3096, Found: 459.3081; specific rotation: $[\alpha]_{\mathrm{D}}{ }^{20}=-158.7\left(c 0.80, \mathrm{CHCl}_{3}\right)$ for an enantiomerically enriched sample of $>99: 1$ e.r.

Enantiomeric purity was determined by HPLC analysis in comparison with authentic racemic material; $(R, R)$-Whelk-O1 column, 99.9:0.1 hexanes/i-PrOH, 0.3 mL/min, $220 \mathrm{~nm}$.
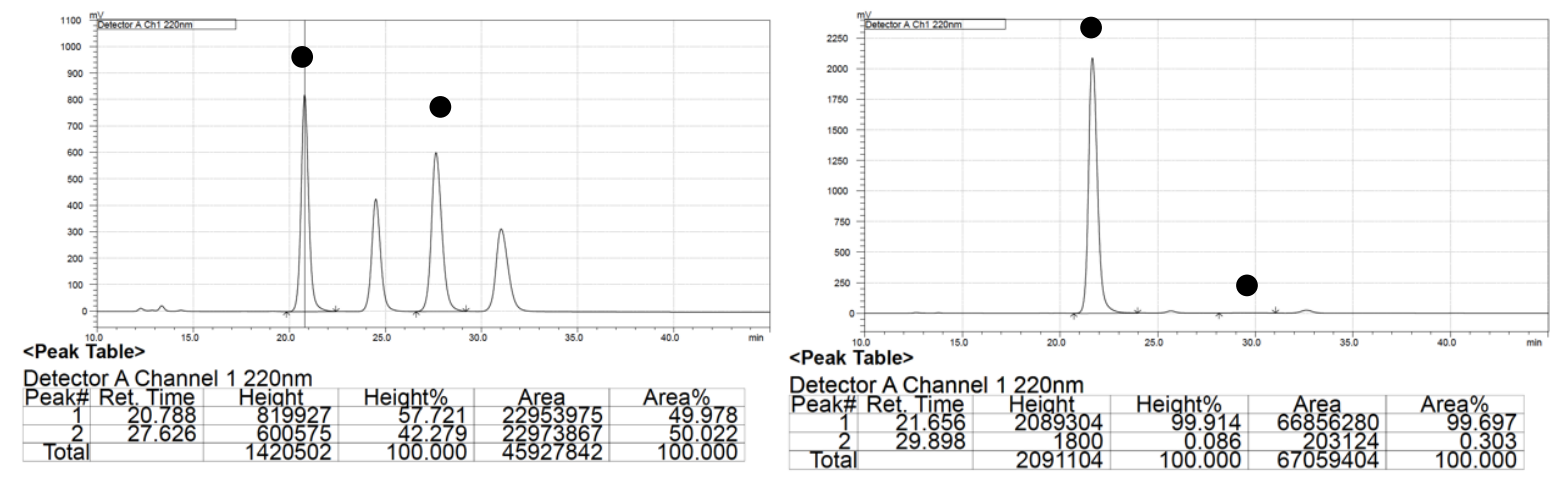

\begin{tabular}{|c|c|c|c|c|c|}
\hline Retention Time & Area & Area\% & Retention Time & Area & Area\% \\
\hline 20.788 & 22953975 & 49.978 & 21.656 & 668566280 & 99.697 \\
\hline 27.626 & 22973867 & 50.022 & 29.898 & 203124 & 0.303 \\
\hline
\end{tabular}

6.4.17 (2S,4R)-4-(2-((t-Butyldimethylsilyl)oxy)ethyl)-2-phenyl-5-(4,4,5,5-tetramethyl1,3,2-dioxaborolan-2-yl)hex-5-en-3-one (4q)<smiles>[M]C(C(=O)[C@@H](CC[SeH])C(=C)Br)c1ccccc1</smiles> 
Modified procedure: $2.0 \mathrm{~mol} \%$ CuMes $(3.6 \mathrm{mg}, 0.040 \mathrm{mmol})$ and $2.2 \mathrm{~mol} \%$ phos-1 (11.9 mg, $0.110 \mathrm{mmol}$ ) was used.

Colorless oil; 413 mg, 0.90 mmol, 90\% yield, 98:2 d.r.; IR (neat): 2953 (w), 2855 (w), 1709 (m), 1310 (m), 1140 (m), 1098 (m), 833 (s), $774(\mathrm{~m}), 698(\mathrm{~m}) \mathrm{cm}^{-1} ;{ }^{1} \mathbf{H}$ NMR (CDCl $\mathbf{3}, \mathbf{5 0 0}$ MHz): $\delta$ 7.29-7.24 (m, 2H), 7.23-7.18 (m, 3H), $5.80(\mathrm{~d}, J=2.8 \mathrm{~Hz}, 1 \mathrm{H}), 5.48(\mathrm{~d}, J=2.8 \mathrm{~Hz}$, $1 \mathrm{H}), 3.94$ (q, $J=7.1 \mathrm{~Hz}, 1 \mathrm{H}), 3.65(\mathrm{t}, J=7.1 \mathrm{~Hz}, 1 \mathrm{H}), 3.58-3.44(\mathrm{~m}, 2 \mathrm{H}), 2.07-1.93(\mathrm{~m}, 1 \mathrm{H})$, $1.86-1.75$ (m, 1H), 1.40 (d, J=7.1 Hz, 3H), $1.21(\mathrm{~s}, 12 \mathrm{H}), 0.90(\mathrm{~s}, 9 \mathrm{H}), 0.03$ (s, 3H), 0.02 (s, $3 \mathrm{H}) ;{ }^{13} \mathbf{C}$ NMR (CDCl 3 , 101 MHz): $\delta$ 211.5, 140.7, 139.1 (C-B, bs), 131.7, 128.6, 128.5,

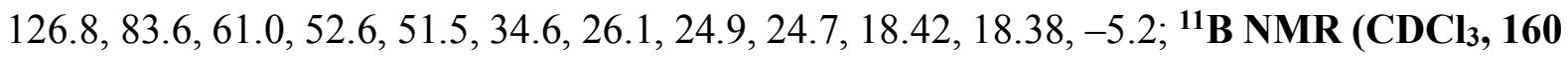
MHz): $\delta 29.7$; HRMS (DART): Calcd for $\mathrm{C}_{26} \mathrm{H}_{44} \mathrm{BO}_{4} \mathrm{Si}[\mathrm{M}+\mathrm{H}]^{+}:$459.3096, Found: 459.3101; specific rotation: $[\alpha]_{\mathrm{D}^{20}}=+10.8\left(c 0.85, \mathrm{CHCl}_{3}\right)$ for an enantiomerically enriched sample of $>99: 1$ e.r.

Enantiomeric purity was determined by HPLC analysis in comparison with authentic racemic material; $(R, R)$-Whelk-O1 column, 99.9:0.1 hexanes/ $i-P r O H, 0.3 \mathrm{~mL} / \mathrm{min}, 220 \mathrm{~nm}$.
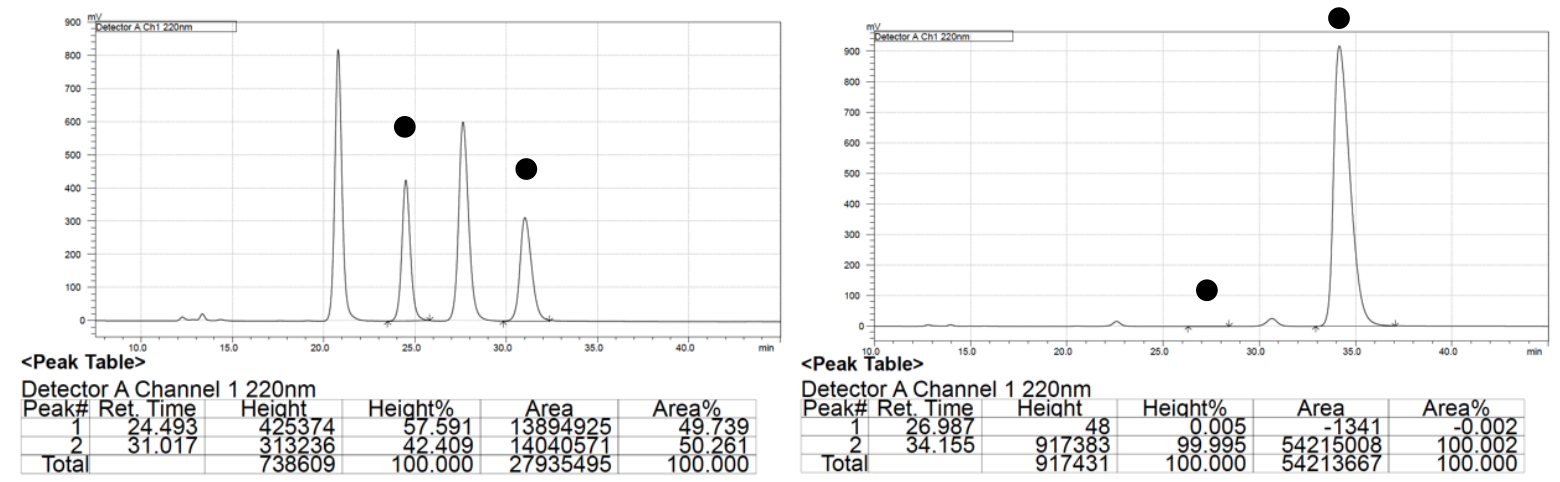

\begin{tabular}{|c|c|c|c|c|c|}
\hline Retention Time & Area & Area $\%$ & Retention Time & Area & Area $\%$ \\
\hline 24.493 & 13894925 & 49.739 & 26.987 & -1341 & -0.002 \\
\hline 31.017 & 14040571 & 50.261 & 34.155 & 54215008 & 100.002 \\
\hline
\end{tabular}

\subsection{Reactions with 1,1-disubstituted allenes}

In a $\mathrm{N}_{2}$-filled glovebox, an oven-dried vial $(4 \mathrm{~mL}, 17 \times 38 \mathrm{~mm})$ was charged with $\mathrm{MesCu}(\mathrm{I})$ (0.9 mg, $0.0050 \mathrm{mmol}),(R)$-DTBM-segphos (7.1 mg, $0.006 \mathrm{mmol})$ and THF $(0.50 \mathrm{~mL})$. The vial was sealed (screw cap) and agitated manually (swirling) until the solution became homogeneous ( $\sim 1 \mathrm{~min})$, after which it was kept for $10 \mathrm{~min}$ at $22{ }^{\circ} \mathrm{C}$. At this time, $i-\operatorname{PrOH}(9.0$ $\mathrm{mg}, 0.15 \mathrm{mmol}$ ) was added by syringe and the mixture was again kept for $10 \mathrm{~min}$ at $22^{\circ} \mathrm{C}$. A separate oven-dried vial was loaded with PhCN (10.3 mg, $0.10 \mathrm{mmol}), t$-Butyldimethyl((2methylbuta-2,3-dien-1-yl)oxy)silane (23.8 mg, $0.12 \mathrm{mmol}$ ), $\mathrm{B}_{2}(\text { pin) })_{2}(30.5 \mathrm{mg}, 0.12 \mathrm{mmol}$ ) and THF $(0.50 \mathrm{~mL})$. The vial was capped and stirred by hand until the solution became homogeneous, after which it was charged, by syringe in a single portion, with the aforementioned solution containing the $\mathrm{Cu}$-based complex. The vial was re-sealed (screw cap) and kept for $6 \mathrm{~h} \mathrm{~h}$ at $22{ }^{\circ} \mathrm{C}$. Analysis of aliquot samples by ${ }^{1} \mathrm{H}$ and ${ }^{11} \mathrm{~B}$ NMR spectroscopy indicated full conversion, after which the reaction was quenched by the addition of $1 \mathrm{~mL}$ of a 1 
$\mathrm{M}$ solution of $\mathrm{HCl}$ followed by vigorous stirring of the mixture for $3 \mathrm{~h}$. The mixture was then washed with $\mathrm{Et}_{2} \mathrm{O}(3 \times 10 \mathrm{~mL})$ and the combined organic layers were dried over $\mathrm{MgSO}_{4}$. Filtration and removal of the volatiles in vacuo afforded light yellow oil, which was purified by silica gel chromatography $\left(20: 1 \rightarrow 15: 1\right.$ hexanes:Et $\left._{2} \mathrm{O}\right)$ to afford $\mathbf{5 a}$ as colorless oil (32.7 $\mathrm{mg}, 0.0760 \mathrm{mmol}, 76 \%$ yield, $94: 6$ e.r.).

\subsection{1 (R)-2-(((t-Butyldimethylsilyl)oxy)methyl)-2-methyl-1-phenyl-3-(4,4,5,5-} tetramethyl-1,3,2-dioxaborolan-2-yl)but-3-en-1-one (5a)<smiles>C=C(Br)C(C)(CO[Sb])C(=O)Nc1ccccc1</smiles>

Colorless oil; $32.7 \mathrm{mg}, 0.0760 \mathrm{mmol}, 76 \%$ yield, 94:6 e.r.; IR (neat): 2975(w), $2854(\mathrm{~m}), 1676$ (s), 1612 (s), 1164 (s), 970 (s) cm ${ }^{-1} ;{ }^{1} \mathbf{H}$ NMR (600 MHz, CDCl $\left.\mathbf{l}_{3}\right): \delta 7.92-7.88$ (m, 2H), $7.44-$ $7.37(\mathrm{~m}, 1 \mathrm{H}), 7.35-7.29(\mathrm{~m}, 2 \mathrm{H}), 6.06(\mathrm{~d}, J=2.5 \mathrm{~Hz}, 1 \mathrm{H}), 5.85(\mathrm{~d}, J=2.5 \mathrm{~Hz}, 1 \mathrm{H}), 4.09$ (dd, $J=9.7,0.9 \mathrm{~Hz}, 1 \mathrm{H}), 3.87(\mathrm{dd}, J=9.8,0.9 \mathrm{~Hz}, 1 \mathrm{H}), 1.47$ (s, 3H), 0.99 (s, 6H), 0.98 (s, 6H), 0.82 (s, 9H), -0.06 (s, 3H), -0.07 (s, 3H); ${ }^{13} \mathbf{C}$ NMR (151 MHz, $\left.\mathbf{C D C l}_{3}\right): \delta 204.2,137.9,131.6$, $129.7,129.3,127.8,83.6,67.3,56.8,26.0,24.6,24.5,22.0,18.4,-5.4,-5.5$; the carbon bearing the boron atom could not be detected due to quadrupolar effects; ${ }^{11} \mathbf{B} \mathbf{N M R}\left(\mathbf{1 9 2} \mathbf{M H z}, \mathbf{C D C l}_{\mathbf{3}}\right)$ : $\delta$ 29.4; HRMS (DART): Calcd for $\mathrm{C}_{24} \mathrm{H}_{39} \mathrm{BO}_{4} \mathrm{Si}[\mathrm{M}+\mathrm{H}]^{+}$: 431.2774, Found: 431.2783; specific rotation: $[\alpha]_{\mathrm{D}}{ }^{20}=-56.9\left(c 1.0, \mathrm{CHCl}_{3}\right)$ for an enantiomerically enriched sample of 94:6 e.r.

Enantiomeric purity was determined by HPLC analysis in comparison with authentic racemic material; $(R, R)-$ Whelk-O1 column, 100:0 hexanes $/ i-\mathrm{PrOH}, 0.5 \mathrm{~mL} / \mathrm{min}, 220 \mathrm{~nm}$.
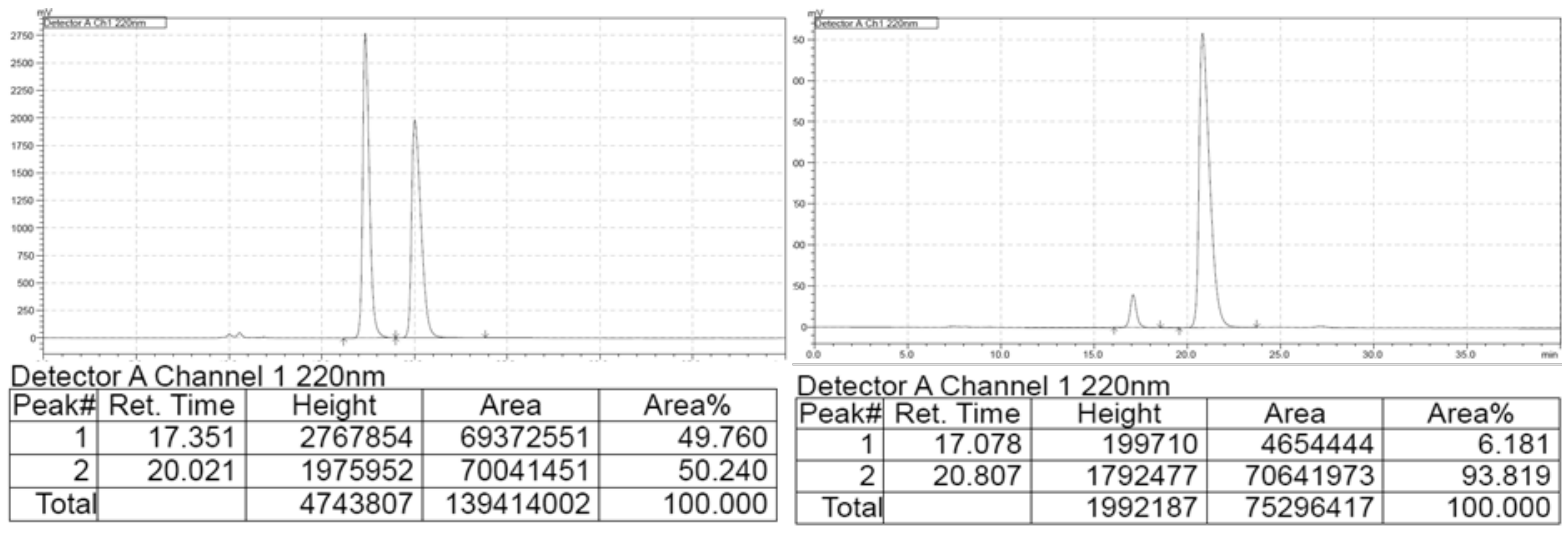

\begin{tabular}{|c|c|c|c|c|c|}
\hline Retention Time & Area & Area\% & Retention Time & Area & Area\% \\
\hline 17.351 & 69372551 & 49.760 & 17.078 & 4654444 & 6.181 \\
\hline 20.021 & 70041451 & 50.240 & 20.807 & 70641973 & 93.819 \\
\hline
\end{tabular}

6.5.2 (R)-1-(Benzo[d][1,3]dioxol-5-yl)-2-(((t-butyldimethylsilyl)oxy)methyl)-2-methyl3-(4,4,5,5-tetramethyl-1,3,2-dioxaborolan-2-yl)but-3-en-1-one (5b)

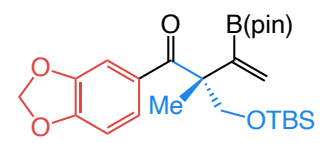


Colorless oil; $24.7 \mathrm{mg}, 0.1040 \mathrm{mmol}, 52 \%$ yield, 93:7 e.r.; IR (neat): 2975(w), 2854 (m), 1676 (s), 1612 (s), 1164 (s), 970 (s) cm ${ }^{-1} ;{ }^{1} \mathbf{H}$ NMR (600 MHz, $\left.\mathbf{C D C l}_{3}\right): \delta 7.57$ (dd, $J=8.2,1.8 \mathrm{~Hz}$, $1 \mathrm{H}), 7.44(\mathrm{~d}, J=1.8 \mathrm{~Hz}, 1 \mathrm{H}), 6.72(\mathrm{~d}, J=8.2 \mathrm{~Hz}, 1 \mathrm{H}), 6.05(\mathrm{~d}, J=2.5 \mathrm{~Hz}, 1 \mathrm{H}), 5.98-5.94$ (m, 2H), $5.83(\mathrm{~d}, J=2.5 \mathrm{~Hz}, 1 \mathrm{H}), 4.05(\mathrm{~d}, J=9.8 \mathrm{~Hz}, 1 \mathrm{H}), 3.84(\mathrm{~d}, J=9.8 \mathrm{~Hz}, 1 \mathrm{H}), 1.44(\mathrm{~s}, 3 \mathrm{H})$, $1.03(\mathrm{~s}, 6 \mathrm{H}), 1.01(\mathrm{~s}, 6 \mathrm{H}), 0.82(\mathrm{~s}, 9 \mathrm{H}),-0.05(\mathrm{~s}, 3 \mathrm{H}),-0.07(\mathrm{~s}, 3 \mathrm{H}) ;{ }^{13} \mathbf{C}$ NMR (151 MHz, $\left.\mathbf{C D C l}_{3}\right): \delta 202.1,150.4,147.4,132.4,129.1,125.9,109.9,107.2,101.5,83.6$, , 67.3, 56.6, $26.0,24.6,24.5,22.1,18.4,-5.3,-5.5$; the carbon bearing the boron atom could not be detected due to quadrupolar effects; ${ }^{11} \mathbf{B}$ NMR (192 $\left.\mathbf{M H z}, \mathbf{C D C l}_{3}\right)$ : $\delta$ 29.4; HRMS (DART): Calcd for $\mathrm{C}_{24} \mathrm{H}_{39} \mathrm{BO}_{4} \mathrm{Si}[\mathrm{M}+\mathrm{H}]^{+}: 431.2774$, Found: 431.2783 ; specific rotation: $[\alpha]_{\mathrm{D}}{ }^{20}=-24.3(c 1.0$, $\mathrm{CHCl}_{3}$ ) for an enantiomerically enriched sample of 93:7 e.r.

Enantiomeric purity was determined by HPLC analysis in comparison with authentic racemic material; $(R, R)$-Whelk-O1 column, 99.5:0.5 hexanes/i-PrOH, $0.5 \mathrm{~mL} / \mathrm{min}, 220 \mathrm{~nm}$.

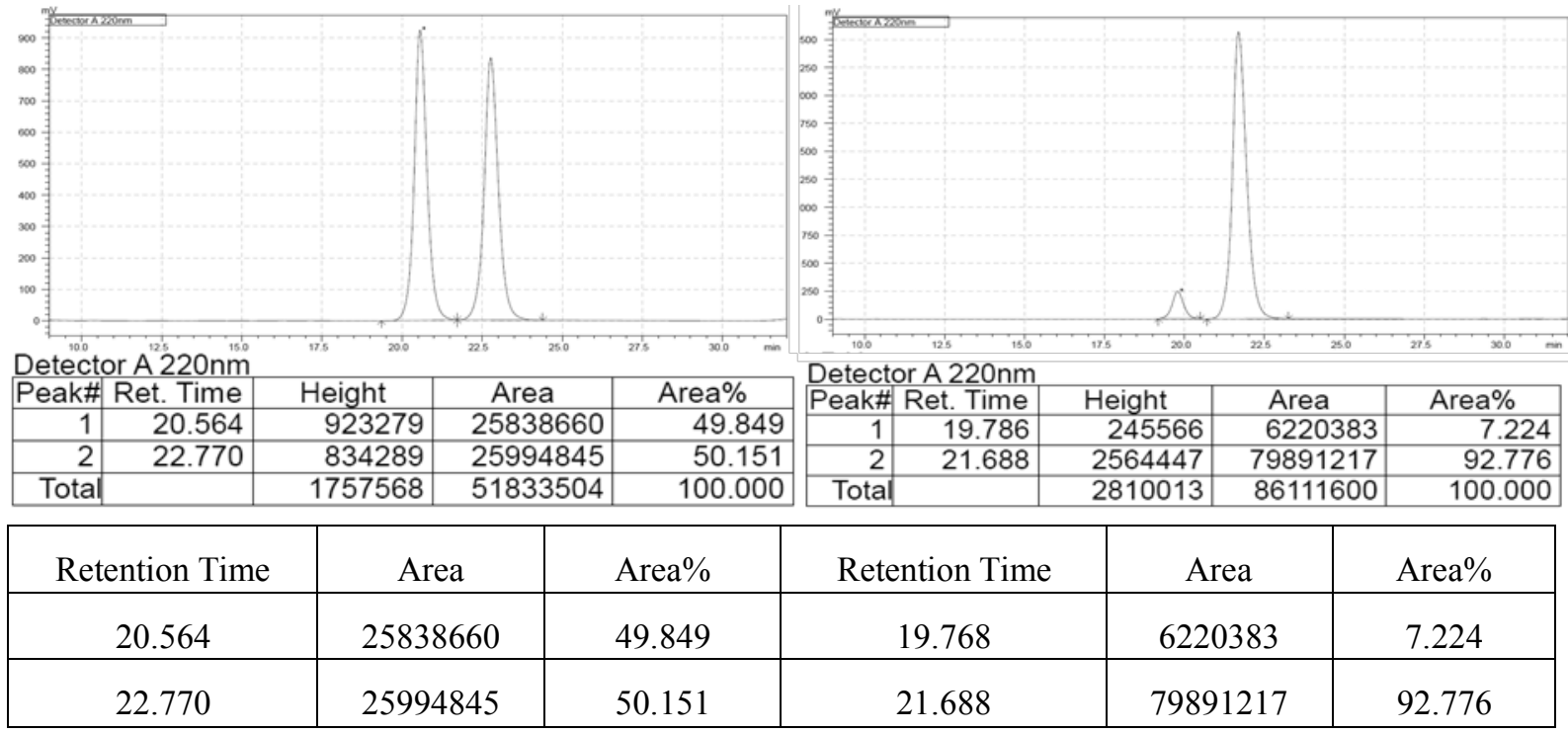

6.5.3 t-Butyl-(R)-3-(2-(((t-butyldimethylsilyl)oxy)methyl)-2-methyl-3-(4,4,5,5tetramethyl-1,3,2-dioxaborolan-2-yl)but-3-enoyl)-1H-indole-1-carboxylate (5c)

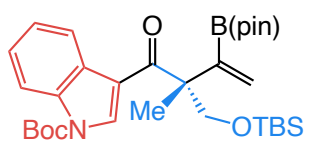

Colorless oil, 32.5 mg, 0.1120 mmol, 56\% yield, 94:6 e.r.; IR (neat): 2974 (w), 2926 (w), 2854 (w), 1737 (s), 1674 (s), 1607 (m), 1413 (w), 910 (s) cm ${ }^{-1}$; ${ }^{1}$ H NMR (500 MHz, CDCl $)$ ): $\delta 8.39$ $(\mathrm{d}, J=9.4 \mathrm{~Hz}, 1 \mathrm{H}), 8.28(\mathrm{~s}, 1 \mathrm{H}), 8.07(\mathrm{~d}, J=7.6 \mathrm{~Hz}, 1 \mathrm{H}), 7.36-7.27(\mathrm{~m}, 2 \mathrm{H}), 6.13(\mathrm{~s}, 1 \mathrm{H})$, $5.93(\mathrm{~s}, 1 \mathrm{H}), 4.10(\mathrm{~s}, 1 \mathrm{H}), 3.92(\mathrm{~d}, J=7.9 \mathrm{~Hz}, 1 \mathrm{H}), 1.66(\mathrm{~s}, 9 \mathrm{H}), 1.46(\mathrm{~s}, 3 \mathrm{H}), 0.88(\mathrm{~s}, 12 \mathrm{H})$, 0.82 (s, 9H), -0.03 (s, 3H), -0.07 (s, 3H); ${ }^{13} \mathbf{C}$ NMR (126 MHz, CDCl $): \delta 200.5,149.3,134.7$, $133.2,129.2,128.7,125.1,124.2,123.3,119.2,114.6,84.8,83.4,67.1,57.5,28.2,26.0,24.5$, $24.4,21.9,18.4,-5.3,-5.5$; the carbon bearing the boron atom could not be detected due to quadrupolar effects; ${ }^{11} \mathrm{~B}$ NMR (192 $\left.\mathbf{M H z}, \mathbf{C D C l}_{3}\right): \delta$ 29.2; HRMS (DART): Calcd for $\mathrm{C}_{31} \mathrm{H}_{49} \mathrm{BNO}_{6} \mathrm{Si}[\mathrm{M}+\mathrm{H}]^{+}: 570.3419$, Found: 570.3416 ; specific rotation: $[\alpha]_{\mathrm{D}}{ }^{20}=-15.0$ (c 1.0, $\mathrm{CHCl}_{3}$ ) for an enantiomerically enriched sample of $94: 6$ e.r. 
Enantiomeric purity was determined by HPLC analysis in comparison with authentic racemic material; $(R, R)$-Whelk-O1 column, 99.5:0.5 hexanes/i-PrOH, $0.5 \mathrm{~mL} / \mathrm{min}, 220 \mathrm{~nm}$.
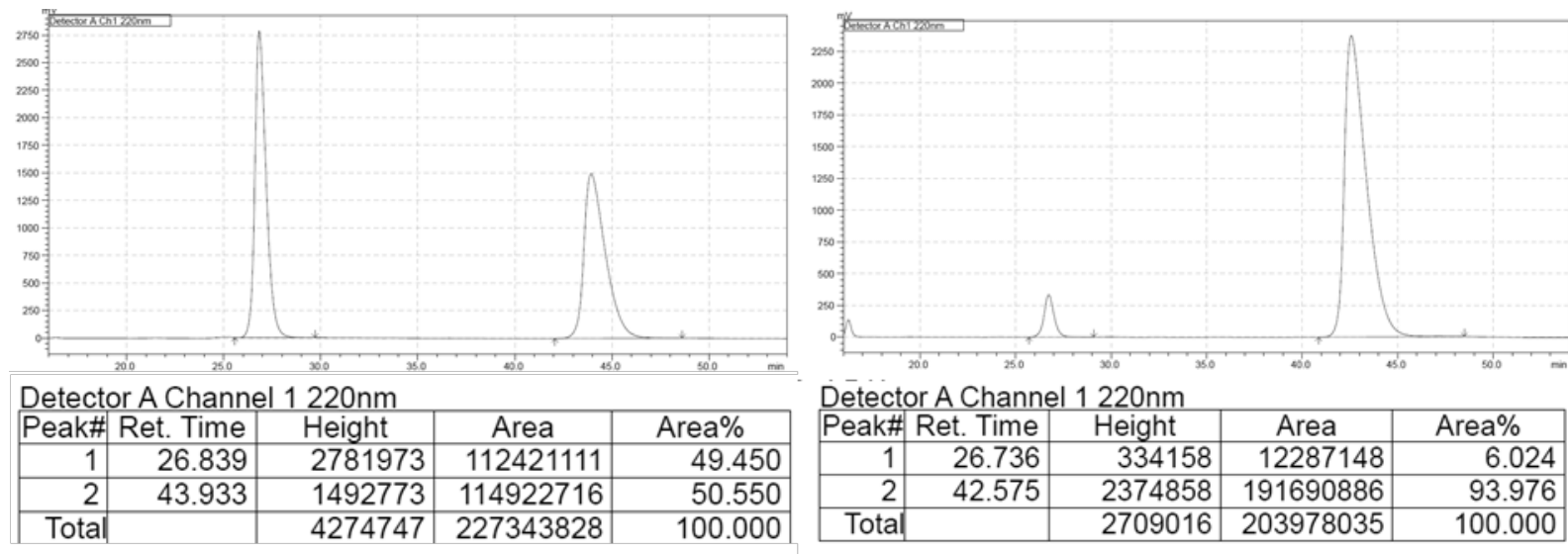

\begin{tabular}{|c|c|c|c|c|c|}
\hline Retention Time & Area & Area $\%$ & Retention Time & Area & Area $\%$ \\
\hline 26.839 & 112421111 & 49.450 & 26.736 & 12287148 & 6.024 \\
\hline 43.933 & 227343828 & 50.550 & 42.575 & 203978035 & 93.976 \\
\hline
\end{tabular}

6.5.4 (R)-3-Methyl-3-phenyl-4-(4,4,5,5-tetramethyl-1,3,2-dioxaborolan-2-yl)pent-4-en2-one (5d)<smiles>C=C(Br)C(C)(Br)c1ccccc1</smiles>

Modified procedure: The reaction was carried out for $6 \mathrm{~h}$ at $22{ }^{\circ} \mathrm{C}$ and was quenched by addition of $1 \mathrm{~mL}$ of a $1 \mathrm{M}$ solution of $\mathrm{HCl}$ followed by vigorous stirring of the mixture for $3 \mathrm{~h}$. Colorless oil; 23.9 mg, 0.0796 mmol, 80\% yield, >99:1 e.r.; IR (neat): 2975 (m), 2931 (w), 1705 (s), 1410 (m), 1370 (s), 1351 (s), 1304 (w), 1213 (w), 1144 (s), 102 (w), 967 (w), 861 (w), $701(\mathrm{~m}) \mathrm{cm}^{-1} ;{ }^{1} \mathbf{H}$ NMR (400 MHz, $\left.\mathbf{C D C l}_{3}\right): \delta$ 7.36-7.29 (m, 2H), 7.29-7.22 (m, 4H), $5.73(\mathrm{~d}$, $J=2.4 \mathrm{~Hz}, 1 \mathrm{H}), 4.90(\mathrm{~d}, J=2.4 \mathrm{~Hz}, 1 \mathrm{H}), 1.97(\mathrm{~s}, 3 \mathrm{H}), 1.78(\mathrm{~s}, 3 \mathrm{H}), 1.28(\mathrm{~s}, 6 \mathrm{H}), 1.26(\mathrm{~s}, 6 \mathrm{H})$; ${ }^{13}$ C NMR (151 MHz, $\left.\mathbf{C D C l}_{3}\right): \delta$ 210.3, 141.1, 128.3, 128.1, 127.8, 126.9, 83.3, 62.5, 26.0, 24.8, 24.5, 22.6; the carbon bearing the boron atom could not be detected due to quadrupolar effects; ${ }^{11} \mathbf{B}$ NMR (160 MHz, $\mathbf{C D C l}_{3}$ ): $\delta 31.2$; HRMS (DART): Calcd for $\mathrm{C}_{18} \mathrm{H}_{26} \mathrm{BO}_{3}[\mathrm{M}+\mathrm{H}]^{+}$: 301.1970, Found: 301.1977; specific rotation: $[\alpha]_{\mathrm{D}}^{20}=-120.6\left(c 1.0, \mathrm{CHCl}_{3}\right)$ for an enantiomerically enriched sample of $>99: 1$ e.r.

Enantiomeric purity was determined by HPLC analysis in comparison with authentic racemic material; $(R, R)$-Whelk-O1 column, 99.9:0.1 hexanes/i-PrOH, $0.3 \mathrm{~mL} / \mathrm{min}, 220 \mathrm{~nm}$. 

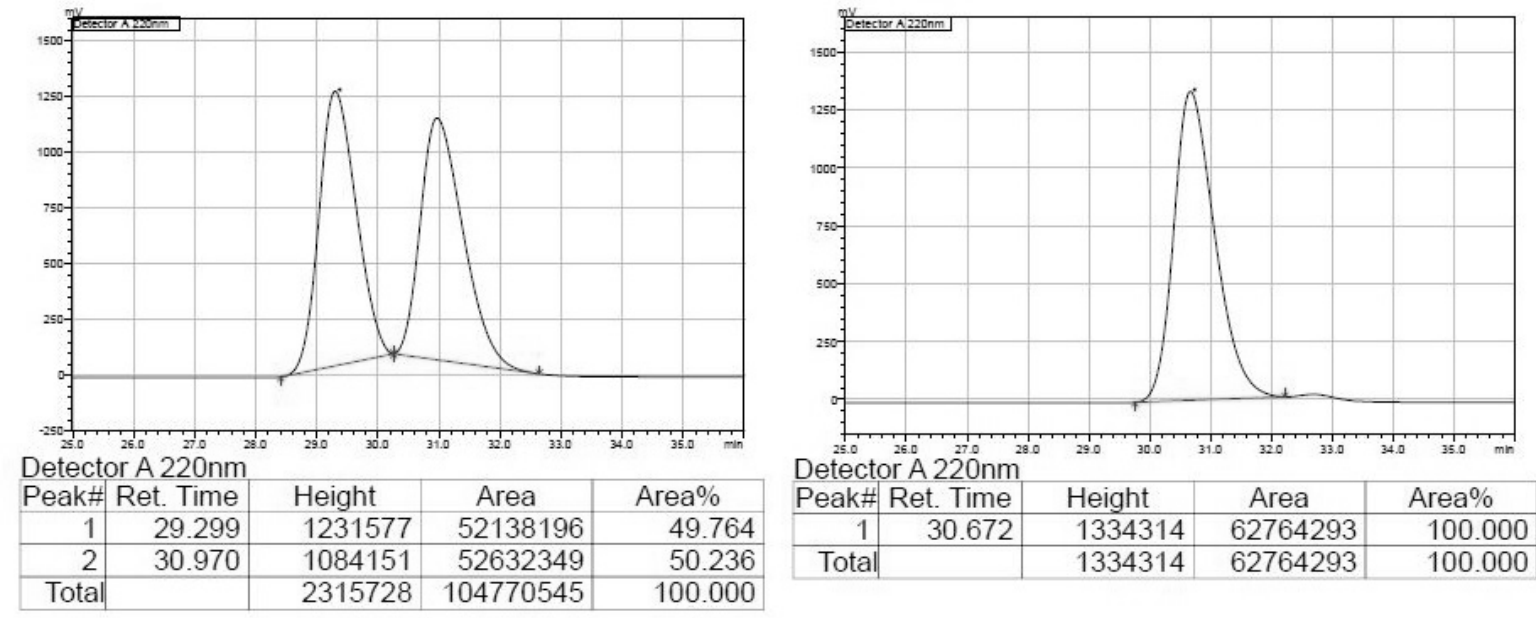

\begin{tabular}{|c|c|c|c|c|c|}
\hline Retention Time & Area & Area $\%$ & Retention Time & Area & Area\% \\
\hline 29.299 & 52138196 & 49.764 & & & \\
\hline 30.970 & 52632349 & 50.236 & 30.672 & 62764293 & 100.000 \\
\hline
\end{tabular}

\subsection{5 (R)-1-Cyclohexyl-2-methyl-2-phenyl-3-(4,4,5,5-tetramethyl-1,3,2-dioxaborolan-}

\section{2-yl)but-3-en-1-one (5e)}<smiles>C=C(Br)[C@](C)(C(=O)Cl)c1ccccc1</smiles>

Modified procedure: $30.1 \mathrm{mg}$ of $i$-PrOH $(0.500 \mathrm{mmol}, 5.0$ equiv. $)$ was used. The reaction was performed for $24 \mathrm{~h}$ at $22{ }^{\circ} \mathrm{C}$ for $24 \mathrm{~h}$ and was quenched by the addition of $1.0 \mathrm{M} \mathrm{HCl}$, after which the mixture was allowed to stir for $8 \mathrm{~h}$ to ensure complete ketimine hydrolysis.

Colorless oil; 20.7 mg, 0.0562 mmol, 56\% yield, 99:1 e.r.; IR (neat): 2974 (m), 2929 (s), 2852 (w), 1696 (s), 1445 (w), 1413 (w), 1370 (m), 1351 (s), 1301 (s), 1133 (s), 1065 (m), 989 (w), $968(\mathrm{w}), 835(\mathrm{w}), 700(\mathrm{w}) \mathrm{cm}^{-1}$; ${ }^{1} \mathbf{H}$ NMR (400 MHz, $\left.\mathbf{C D C l}_{3}\right): \delta$ 7.34-7.21 (m, 5H), $5.65(\mathrm{~d}$, $J=2.6 \mathrm{~Hz}, 1 \mathrm{H}), 4.77(\mathrm{~d}, J=2.6 \mathrm{~Hz}, 1 \mathrm{H}), 2.4-2.38(\mathrm{~m}, 1 \mathrm{H}), 1.81(\mathrm{~s}, 3 \mathrm{H}), 1.76-1.64(\mathrm{~m}, 2 \mathrm{H})$, 1.59-1.49 (m, 2H), 1.39-1.30 (m, 2H), 1.29 (s, 6H), 1.26 (s, 6H), 1.19-1.09 (m, 2H), 1.08$1.01(\mathrm{~m}, 1 \mathrm{H}), 1.00-0.87(\mathrm{~m}, 1 \mathrm{H}) ;{ }^{13} \mathbf{C} \mathbf{N M R}\left(\mathbf{1 0 1} \mathbf{M H z}, \mathbf{C D C l}_{3}\right): \delta 214.8,140.0,128.6,128.1$, $126.95,126.87,83.2,63.1,46.2,30.5,30.4,25.7,25.6,25.5,25.1,24.4,21.7$; the carbon bearing the boron atom could not be detected due to quadrupolar effects; ${ }^{11} \mathbf{B}$ NMR (160 MHz, CDCl $_{3}$ ): $\delta$ 31.0; HRMS (DART): Calcd for $\mathrm{C}_{23} \mathrm{H}_{34} \mathrm{BO}_{3}[\mathrm{M}+\mathrm{H}]^{+}:$369.2596, Found: 369.2591; specific rotation: $[\alpha]_{\mathrm{D}}{ }^{20}=-119.8\left(c 1.0, \mathrm{CHCl}_{3}\right)$ for an enantiomerically enriched sample of 99:1 e.r.

Enantiomeric purity was determined by HPLC analysis in comparison with authentic racemic material; $(R, R)$-Whelk-O1 column, hexanes, $0.3 \mathrm{~mL} / \mathrm{min}, 220 \mathrm{~nm}$. 

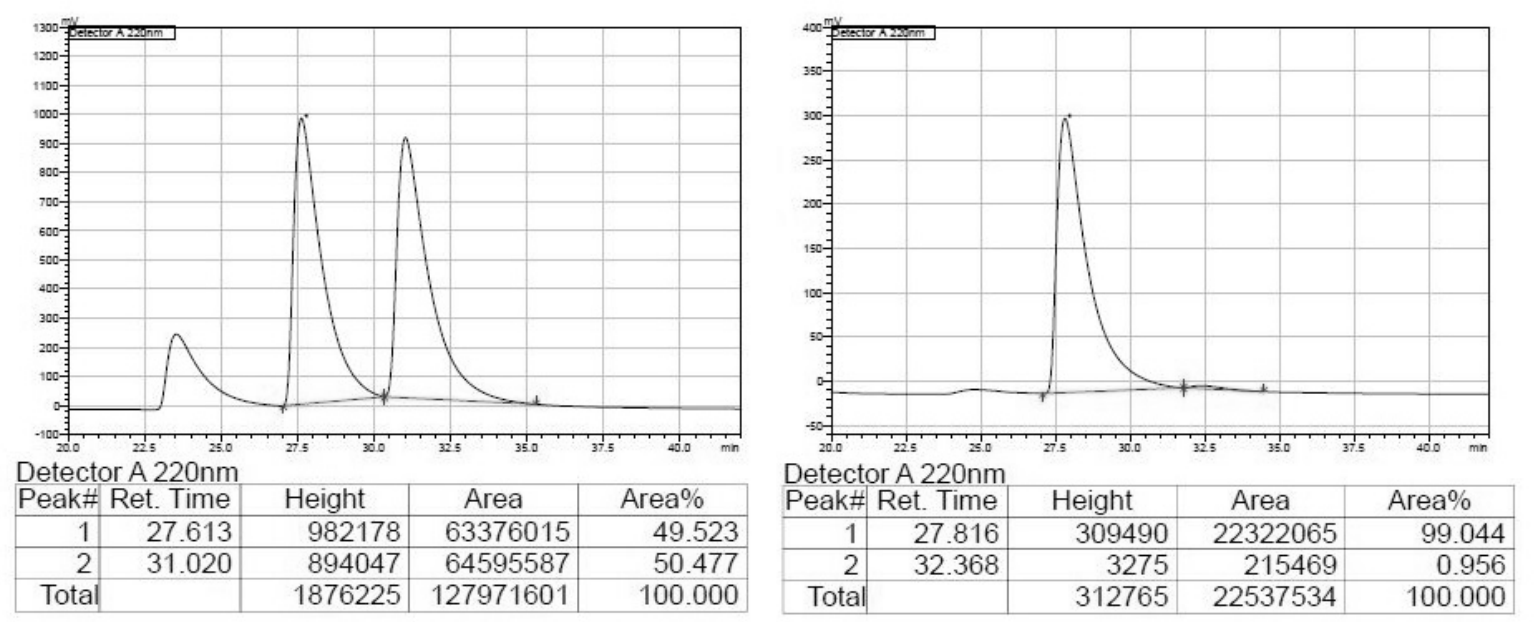

\begin{tabular}{|c|c|c|c|c|c|}
\hline Retention Time & Area & Area $\%$ & Retention Time & Area & Area\% \\
\hline 27.613 & 63376015 & 49.523 & 27.816 & 22322065 & 99.044 \\
\hline 31.020 & 64595587 & 50.477 & 32.368 & 215469 & 0.956 \\
\hline
\end{tabular}

\subsection{6 (R)-4-Methyl-4-phenyl-5-(4,4,5,5-tetramethyl-1,3,2-dioxaborolan-2-yl)-1-} (trimethylsilyl)hex-5-en-1-yn-3-one (5f)

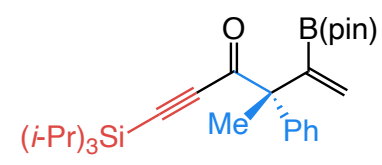

Modified procedure: the reaction was performed for $16 \mathrm{~h}$ at $-40{ }^{\circ} \mathrm{C}$ and was quenched by addition of a saturated aqueous solution of $\mathrm{NH}_{4} \mathrm{Cl}$.

Colorless solid; $35.9 \mathrm{mg}, 0.094 \mathrm{mmol}, 94 \%$ yield, 97.5:2.5 e.r.; IR (neat): 2943 (m), 2866 (m), 2150 (w), 1668 (s), 1351 (s), 1142 (s), 1043 (s), 678 (m) cm ${ }^{-1}$; ${ }^{1}$ H NMR (400 MHz, CDCl 3 ): $\delta 7.35-7.18(\mathrm{~m}, 5 \mathrm{H}), 5.80(\mathrm{~d}, J=2.4 \mathrm{~Hz}, 1 \mathrm{H}), 5.02(\mathrm{~d}, J=2.4 \mathrm{~Hz}, 1 \mathrm{H}), 1.89(\mathrm{~s}, 3 \mathrm{H}), 1.27(\mathrm{~s}$, 6H), 1.26 (s, 6H), 0.98-0.92 (m, 21H); ${ }^{13} \mathbf{C}$ NMR (101 MHz, CDCl 3$):$ 189.4, 140.4, 129.3, $128.8,128.3,127.2,103.2,98.1,83.6,62.6,24.9,24.7,22.8,18.5,11.0$; the carbon bearing the boron atom could not be detected due to quadrupolar effects; ${ }^{11} \mathbf{B}$ NMR (128 $\left.\mathbf{M H z}, \mathbf{C D C l}_{3}\right): \delta$ 29.7; HRMS (DART): Calcd for $\mathrm{C}_{23} \mathrm{H}_{28} \mathrm{BO}_{3}[\mathrm{M}+\mathrm{H}]^{+}$: 467.3153, Found: 467.3175; specific rotation: $[\alpha]_{\mathrm{D}^{20}}=+61.0\left(c 1.0, \mathrm{CHCl}_{3}\right)$ for an enantiomerically enriched sample of 97.5:2.5 e.r.

Enantiomeric purity was determined by HPLC analysis in comparison with authentic racemic material; Chiralcel OD-H column, 99.5:0.5 hexanes $/ i$-PrOH, $0.5 \mathrm{~mL} / \mathrm{min}, 210 \mathrm{~nm}$. 

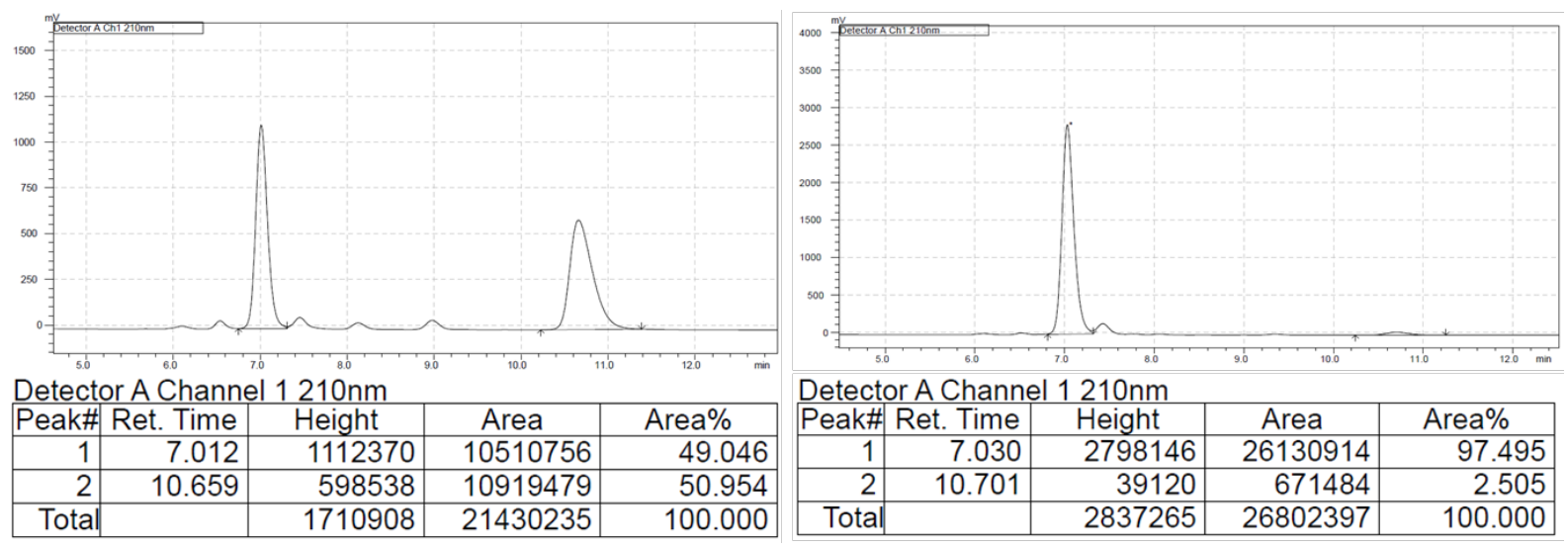

\begin{tabular}{|c|c|c|c|c|c|}
\hline Retention Time & Area & Area\% & Retention Time & Area & Area\% \\
\hline 7.012 & 10510756 & 49.046 & 7.030 & 26130914 & 97.495 \\
\hline 10.659 & 10919479 & 50.954 & 10.701 & 671484 & 2.505 \\
\hline
\end{tabular}

6.5.7 (R)-2-Methyl-1,2-diphenyl-3-(4,4,5,5-tetramethyl-1,3,2-dioxaborolan-2-yl)but-3en-1-one (5g)

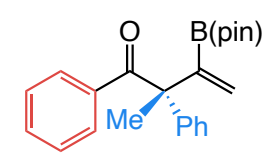

Modified procedure: The reaction was carried out for $16 \mathrm{~h}$ at $22{ }^{\circ} \mathrm{C}$ and was quenched by addition of $1 \mathrm{~mL}$ of a $1 \mathrm{M}$ solution of $\mathrm{HCl}$ followed by vigorous stirring of the mixture for $3 \mathrm{~h}$. Colorless solid; $30.1 \mathrm{mg}, 0.083 \mathrm{mmol}, 83 \%$ yield, 98:2 e.r.; IR (neat): 2977 (m), $2922(\mathrm{w})$, 1671 (m), 1371 (s), 1216 (s), 967 (s), 760 (w) cm ${ }^{-1} ;{ }^{1}$ H NMR (500 MHz, CDCl 3 ): $\delta$ 7.48-7.40 (m, 2H), 7.39-7.31 (m, 5H), 7.32-7.27 (m, 1H), 7.25-7.20 (m, 2H), $5.73(\mathrm{~s}, 1 \mathrm{H}), 4.79(\mathrm{~s}, 1 \mathrm{H})$, 1.87 (s, 3H), 1.32 (s, 12H); ${ }^{13}$ C NMR (101 MHz, CDCl 3$): \delta 204.2,150.3$ (C-B bs), 141.6, $137.3,131.6,129.5,128.7,128.3,128.0,127.2,127.0,83.4,61.3,29.9,25.2,24.6,24.3 ;{ }^{11} \mathbf{B}$ NMR (160 MHz, CDCl 3 ): $\delta 33.1$; HRMS (DART): Calcd for $\mathrm{C}_{23} \mathrm{H}_{28} \mathrm{BO}_{3}[\mathrm{M}+\mathrm{H}]^{+}: 363.2132$, Found: 363.2148 ; specific rotation: $[\alpha]_{\mathrm{D}}{ }^{20}=-126.2\left(c 1.0, \mathrm{CHCl}_{3}\right)$ for an enantiomerically enriched sample of 98:2 e.r.

Enantiomeric purity was determined by HPLC analysis in comparison with authentic racemic material; Chiralcel OD-H column, 99.5:0.5 hexanes $/ i-\mathrm{PrOH}, 0.5 \mathrm{~mL} / \mathrm{min}, 254 \mathrm{~nm}$.
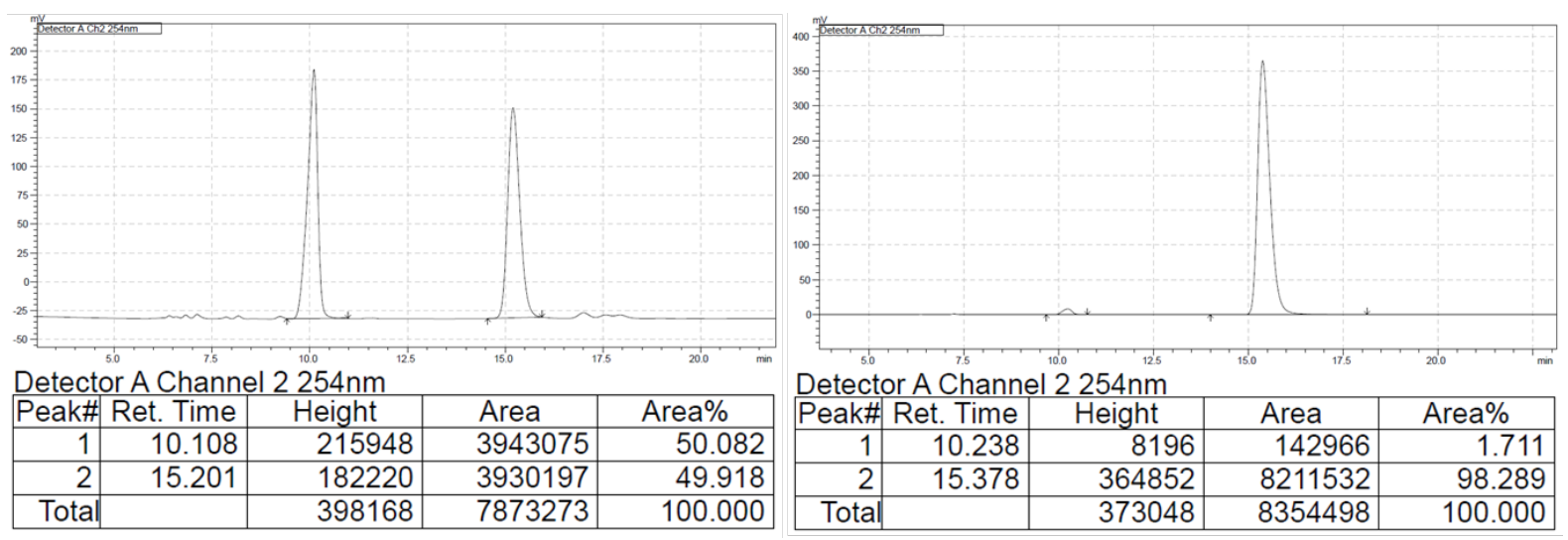

Retention Time Area Area $\%$ Retention Time Area Area\% 


\begin{tabular}{|l|l|l|l|l|l|}
\hline 10.108 & 3943075 & 50.082 & 10.238 & 142966 & 1.711 \\
\hline 15.201 & 3930197 & 49.918 & 15.378 & 8211532 & 98.289 \\
\hline
\end{tabular}

\subsection{Reactions with $\boldsymbol{O}$-substituted allenes}

Modified procedure was followed: the reaction was quenched by the addition of $0.1 \mathrm{M} \mathrm{HCl}$.

\subsection{1 (R)-1-Phenyl-3-(4,4,5,5-tetramethyl-1,3,2-dioxaborolan-2-yl)-2-}

((triisopropylsilyl)oxy)but-3-en-1-one (6a)<smiles></smiles>

Colorless oil; $39.6 \mathrm{mg}, 0.080 \mathrm{mmol}, 80 \%$ yield, 96:4 e.r.; IR (neat): 2925 (m), 2854 (w), 1680 (s), 1620 (m), 1553 (m), 832 (s) cm-1; ${ }^{1} \mathbf{H}$ NMR (600 MHz, CDCl $)$ ): $\delta$ 8.06-8.03 (m, 2H), 7.49-7.44 (m, 1H), 7.38-7.33 (m, 2H), $6.33(\mathrm{~s}, 1 \mathrm{H}), 6.08(\mathrm{~s}, 1 \mathrm{H}), 5.56(\mathrm{~s}, 1 \mathrm{H}), 1.19(\mathrm{~s}, 6 \mathrm{H})$, 1.18 (s, 6H), 1.17-1.09 (m, 3H), 1.06-0.97 (m, 18H); ${ }^{13} \mathbf{C}$ NMR (151 MHz, CDCl 3 ): $\delta$ 198.7, $135.7,132.6,131.9,129.7,128.1,83.9,78.7,24.9,24.6,18.1,17.9,12.3$; the carbon bearing the boron atom could not be detected due to quadrupolar effects; ${ }^{11} \mathbf{B} \mathbf{N M R}\left(\mathbf{1 9 2} \mathbf{M H z}, \mathbf{C D C l}_{3}\right)$ : $\delta$ 29.8; HRMS (DART): Calcd for $\mathrm{C}_{26} \mathrm{H}_{40} \mathrm{BO}_{5} \mathrm{Si}[\mathrm{M}+\mathrm{H}]^{+}:$471.2724, Found: 471.2736; specific rotation: $[\alpha]_{\mathrm{D}}{ }^{20}=-36.5\left(c 0.2, \mathrm{CHCl}_{3}\right)$ for an enantiomerically enriched sample of 96:4 e.r.

Enantiomeric purity was determined by HPLC analysis in comparison with authentic racemic material; $(R, R)$-Whelk-O1 column, pure hexanes, $0.5 \mathrm{~mL} / \mathrm{min}, 220 \mathrm{~nm}$.
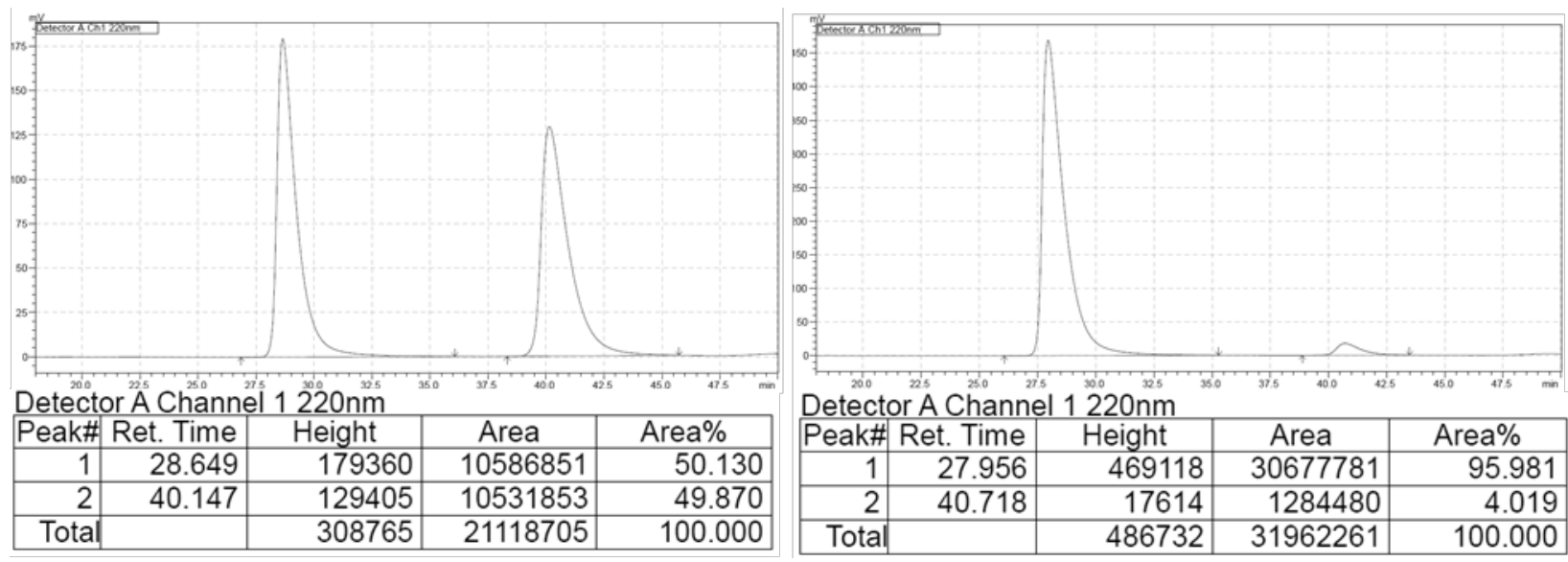

\begin{tabular}{|c|c|c|c|c|c|}
\hline Retention Time & Area & Area\% & Retention Time & Area & Area\% \\
\hline 40.595 & 126437860 & 48.961 & 41.583 & 112655741 & 95.935 \\
\hline 47.279 & 131803347 & 51.039 & 49.710 & 4773574 & 4.065 \\
\hline
\end{tabular}

6.6.2 (R)-3-(Benzyloxy)-2-(4,4,5,5-tetramethyl-1,3,2-dioxaborolan-2-yl)non-1-en-8-yn4-one (6b)<smiles>C#CCCCC(=O)C(Br)C(=C)Br</smiles> 
Colorless oil, $31.7 \mathrm{mg}, 0.086 \mathrm{mmol}, 86 \%$ yield, 97:3 e.r.; IR (neat): 3289 (w), 2974 (w), 2928 (w), 1714 (s), 1661 (s), 1516 (s), 1313 (s) 697 (s) cm ${ }^{-1}$; ${ }^{1}$ H NMR (600 MHz, CDCl $)$ ) $\delta$ 7.39$7.30(\mathrm{~m}, 4 \mathrm{H}), 7.3-7.26(\mathrm{~m}, 1 \mathrm{H}), 6.09(\mathrm{~d}, J=2.9,1 \mathrm{H}), 6.00(\mathrm{~d}, J=2.9,1 \mathrm{H}), 4.62(\mathrm{~d}, J=11.9$ $\mathrm{Hz}, 1 \mathrm{H}), 4.45(\mathrm{~d}, J=11.9 \mathrm{~Hz}, 1 \mathrm{H}), 4.46(\mathrm{~s}, 1 \mathrm{H}), 2.83-2.79(\mathrm{~m}, 1 \mathrm{H}), 2.71-2.67(\mathrm{~m}, 1 \mathrm{H}), 2.24-$ $2.16(\mathrm{~m}, 2 \mathrm{H}), 1.92(\mathrm{t}, J=2.6 \mathrm{~Hz}, 1 \mathrm{H}), 1.79-1.74(\mathrm{~m}, 2 \mathrm{H}), 1.26(\mathrm{~s}, 6 \mathrm{H}), 1.26(\mathrm{~s}, 6 \mathrm{H}) ;{ }^{13} \mathbf{C ~ N M R}$ (151 MHz, $\left.\mathbf{C D C l}_{3}\right): \delta 209.2,138.0,132.5,128.5,127.9,127.8,86.6,84.1,84.0,71.2,68.9$, 37.0, 25.0, 24.7, 22.2, 18.0; the carbon bearing the boron atom could not be detected due to quadrupolar effects; ${ }^{11} \mathbf{B}$ NMR (192 $\left.\mathbf{M H z}, \mathbf{C D C l}_{\mathbf{3}}\right)$ : $\delta$ 29.3; HRMS (DART): Calcd for $\mathrm{C}_{22} \mathrm{H}_{30} \mathrm{BO}_{4}[\mathrm{M}+\mathrm{H}]^{+}:$369.2232, Found: 369.2232 ; specific rotation: $[\alpha]_{\mathrm{D}}{ }^{20}=-36.7$ (c 1.0, $\mathrm{CHCl}_{3}$ ) for an enantiomerically enriched sample of $97: 3$ e.r.

Enantiomeric purity was determined by HPLC analysis in comparison with authentic racemic material; $(R, R)$-Whelk-O1 column, 99.0:1.0 hexanes/i-PrOH, $0.5 \mathrm{~mL} / \mathrm{min}, 220 \mathrm{~nm}$.

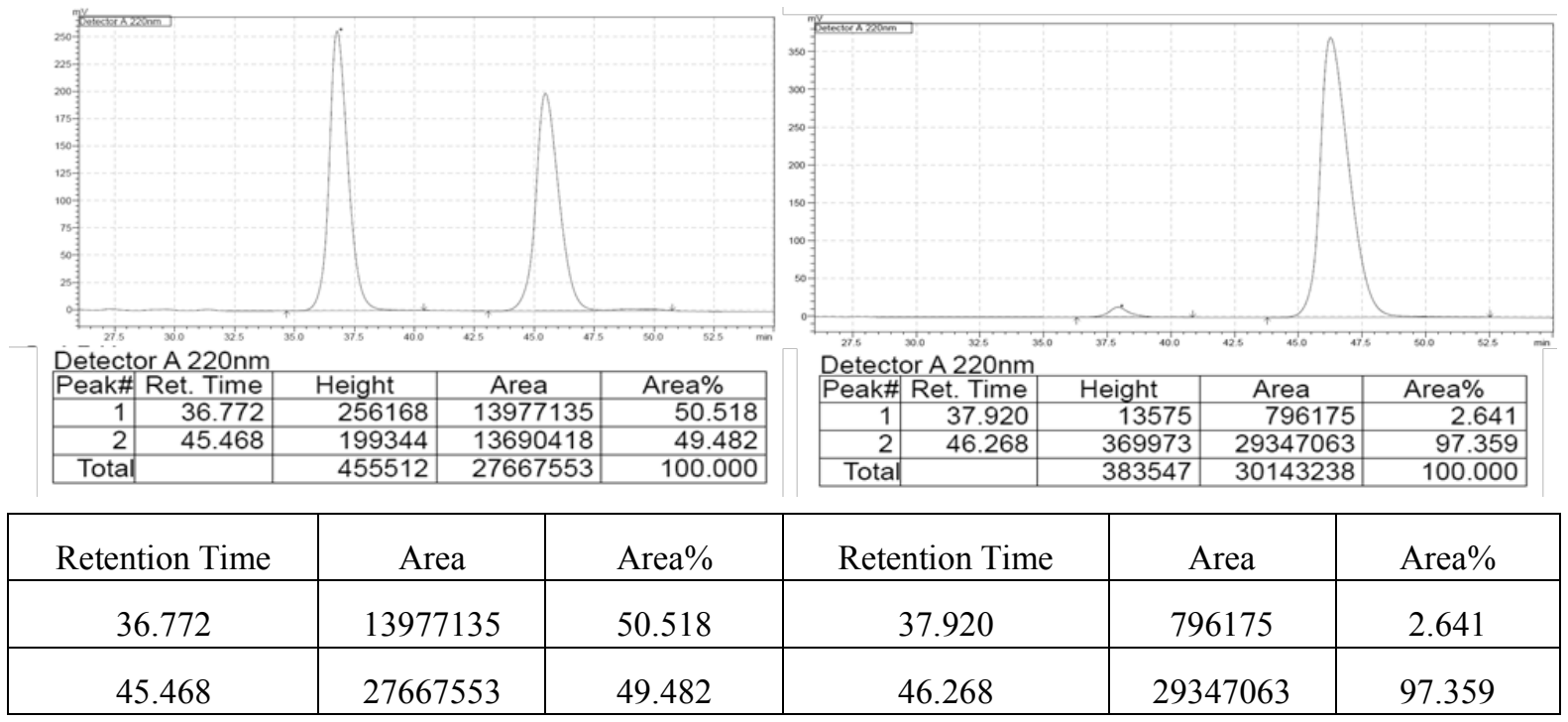

6.6.3 (R)-3-(Benzyloxy)-2-(4,4,5,5-tetramethyl-1,3,2-dioxaborolan-2-yl)hepta-1,6-dien4-one (6c)<smiles>C=CCC(=O)C(Br)C(=C)Br</smiles>

Colorless oil; 20.9 mg, 0.0610 mmol, 61\% yield, 97:3 e.r.; IR (neat): 2975 (m), 1718 (s), 1640 (m), 1618 (w), 1314 (s), 1139 (s) cm ${ }^{-1} ;{ }^{1} \mathbf{H}$ NMR (600 MHz, CDCl $)$ : $\delta$ 7.40-7.32 (m, 4H), $7.32-7.28(\mathrm{~m}, 1 \mathrm{H}), 6.10(\mathrm{~d}, J=2.9 \mathrm{~Hz}, 1 \mathrm{H}), 6.01(\mathrm{~d}, J=2.9 \mathrm{~Hz}, 1 \mathrm{H}), 5.93-5.89(\mathrm{~m}, 1 \mathrm{H}), 5.17-$ $5.13(\mathrm{~m}, 1 \mathrm{H}), 5.12-5.06(\mathrm{~m}, 1 \mathrm{H}), 4.61(\mathrm{~d}, J=11.8 \mathrm{~Hz}, 1 \mathrm{H}), 4.50(\mathrm{~d}, J=1.3 \mathrm{~Hz}, 1 \mathrm{H}), 4.45(\mathrm{~d}$, $J=11.8 \mathrm{~Hz}, 1 \mathrm{H}), 3.48-3.34(\mathrm{~m}, 2 \mathrm{H}), 1.26(\mathrm{~s}, 12 \mathrm{H}) ;{ }^{13} \mathbf{C}$ NMR (151 MHz, CDCl $\left.\mathbf{3}\right): \delta 207.5$, $137.9,132.7,130.9,128.5,127.9,118.6,86.4,84.0,71.2,43.1,25.0,24.7$; the carbon bearing the boron atom could not be detected due to quadrupolar effects; ${ }^{11} \mathbf{B} \mathbf{N M R}\left(\mathbf{1 9 2} \mathbf{M H z}, \mathbf{C D C l}_{\mathbf{3}}\right)$ : $\delta$ 29.4; HRMS (DART): Calcd for $\mathrm{C}_{20} \mathrm{H}_{28} \mathrm{BO}_{4}[\mathrm{M}+\mathrm{H}]^{+}:$343.2072, Found: 343.2075; specific rotation: $[\alpha]_{\mathrm{D}}{ }^{20}=-19.6\left(c 1.0, \mathrm{CHCl}_{3}\right)$ for an enantiomerically enriched sample of 97:3 e.r.

Enantiomeric purity was determined by HPLC analysis in comparison with authentic racemic material; $(R, R)$-Whelk-O1 column, 99.0:1.0 hexanes/i-PrOH, $0.5 \mathrm{~mL} / \mathrm{min}, 220 \mathrm{~nm}$. 


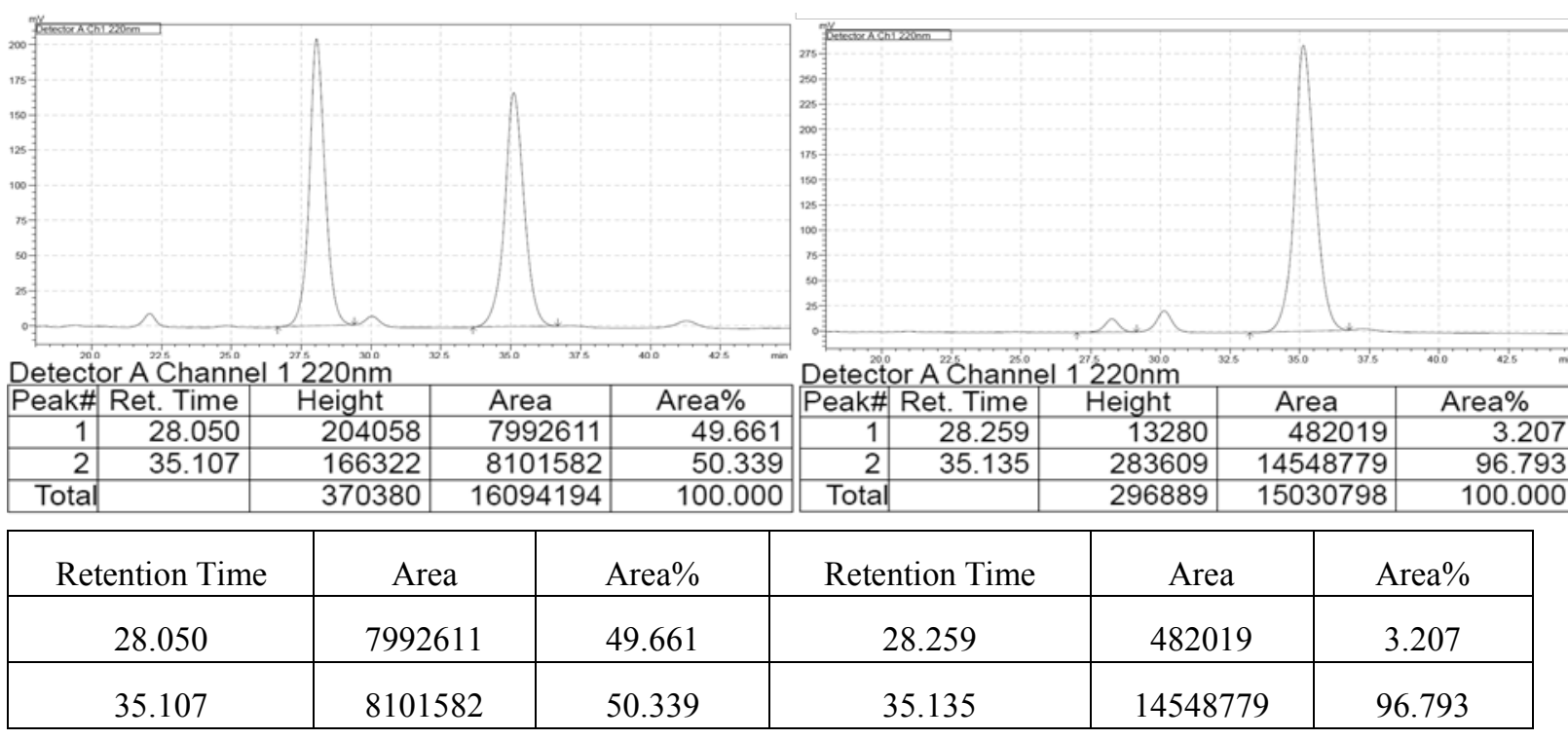

6.6.4 Methyl (R)-5-(benzyloxy)-4-oxo-6-(4,4,5,5-tetramethyl-1,3,2-dioxaborolan-2yl)hept-6-enoate (6d)<smiles>C=C(Br)C(Br)(Br)c1ccccc1</smiles>

Colorless oil; $32.6 \mathrm{mg}, 0.1680$ mmol, 84\% yield, 97:3 e.r.; IR (neat): 2976 (w), 1737 (s), 1719 (s), 1357 (s), 1166 (s) cm ${ }^{-1}$; ${ }^{1} \mathbf{H}$ NMR (600 MHz, CDCl $): \delta 7.40-7.32$ (m, 4H), 7.32-7.26 $(\mathrm{m}, 1 \mathrm{H}), 6.09(\mathrm{~d}, J=2.9,1 \mathrm{H}), 6.00(\mathrm{~d}, J=2.9,1 \mathrm{H}), 4.61(\mathrm{~d}, J=11.8 \mathrm{~Hz}, 1 \mathrm{H}), 4.49(\mathrm{~s}, 1 \mathrm{H})$, $4.47(\mathrm{~d}, J=11.8 \mathrm{~Hz}, 1 \mathrm{H}), 3.65(\mathrm{~s}, 3 \mathrm{H}), 3.12-2.83(\mathrm{~m}, 2 \mathrm{H}), 2.69-2.41(\mathrm{~m}, 2 \mathrm{H}), 1.25(\mathrm{~s}, 12 \mathrm{H})$; ${ }^{13}$ C NMR (151 MHz, CDCl 3$): ~ \delta 208.1,173.4,137.9,132.7,128.5,127.83,127.81,86.5,84.0$, $71.2,51.8,33.4,27.7,24.9,24.7$; the carbon bearing the boron atom could not be detected due to quadrupolar effects; ${ }^{11} \mathbf{B}$ NMR (192 $\left.\mathbf{M H z}, \mathbf{C D C l}_{3}\right): \delta 29.5$; HRMS (DART): Calcd for $\mathrm{C}_{21} \mathrm{H}_{30} \mathrm{BO}_{6}[\mathrm{M}+\mathrm{H}]^{+}:$389.2135, Found: 389.2130 ; specific rotation: $[\alpha]_{\mathrm{D}}{ }^{20}=-23.4(c$ 1.0, $\mathrm{CHCl}_{3}$ ) for an enantiomerically enriched sample of 97:3 e.r.

Enantiomeric purity was determined by HPLC analysis in comparison with authentic racemic material; $(R, R)$-Whelk-O1 column, 95.0:5.0 hexanes/i-PrOH, $0.5 \mathrm{~mL} / \mathrm{min}, 220 \mathrm{~nm}$.

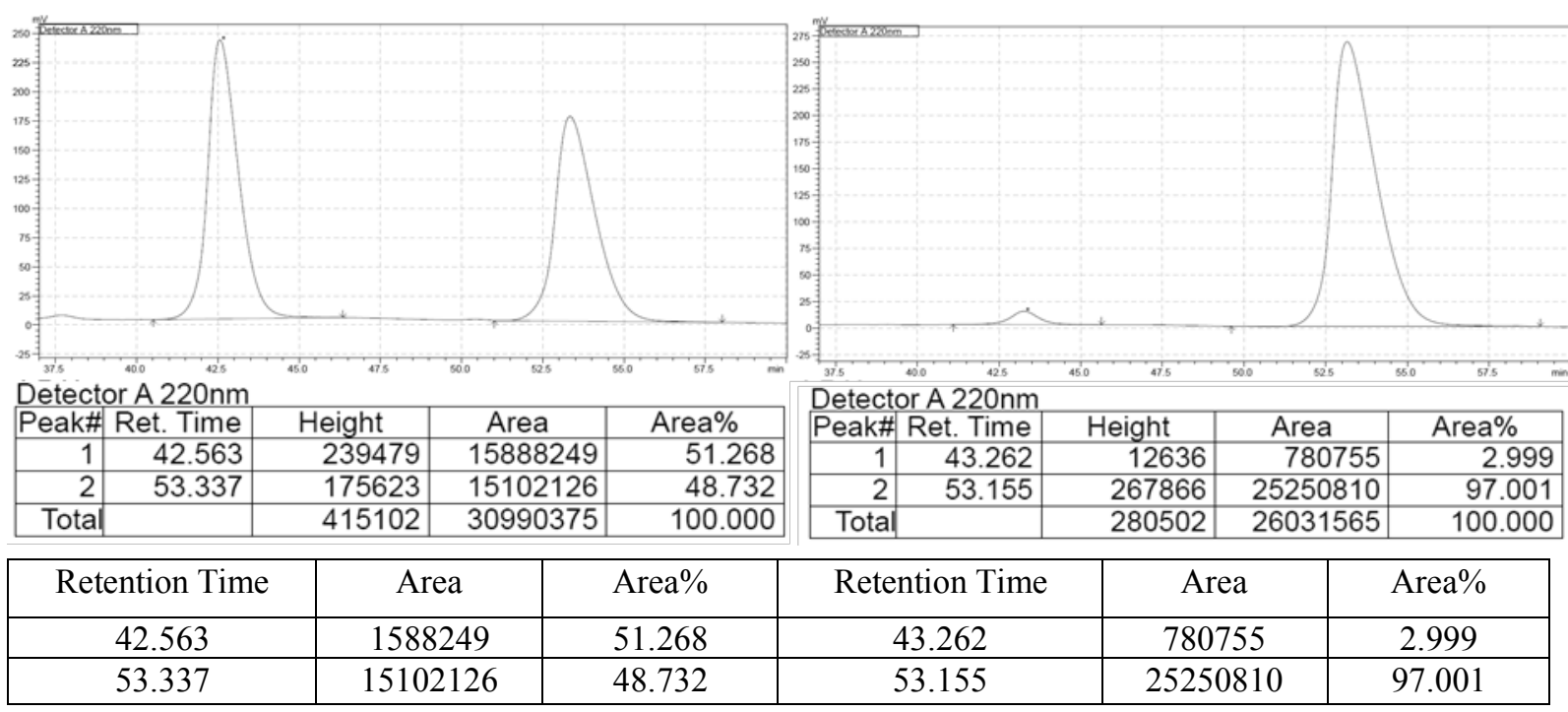


6.6.5 (R)-4-(Benzyloxy)-5-(4,4,5,5-tetramethyl-1,3,2-dioxaborolan-2-yl)hex-5-en-3-one (6e)<smiles>C=C(Br)C(Br)C(=O)CC</smiles>

Colorless oil; $26.4 \mathrm{mg}, 0.0800 \mathrm{mmol}, 80 \%$ yield, 96.5:3.5 e.r.; IR (neat): 2975 (m), 1716 (s), 1314 (s), 1165 (s), 698 (m), cm ${ }^{-1}$; ${ }^{1}$ H NMR (600 MHz, CDCl $): \delta 7.41-7.32$ (m, 4H), 7.32$7.27(\mathrm{~m}, 1 \mathrm{H}), 6.07(\mathrm{~d}, J=2.9 \mathrm{~Hz}, 1 \mathrm{H}), 5.99$ (d, $J=2.9 \mathrm{~Hz}, 1 \mathrm{H}), 4.61$ (d, $J=11.8 \mathrm{~Hz}, 1 \mathrm{H}), 4.48$ $(\mathrm{s}, 1 \mathrm{H}), 4.45(\mathrm{~d}, J=11.8 \mathrm{~Hz}, 1 \mathrm{H}), 2.75-2.64(\mathrm{~m}, 1 \mathrm{H}), 2.62-2.50(\mathrm{~m}, 1 \mathrm{H}), 1.26(\mathrm{~s}, 6 \mathrm{H}), 1.25(\mathrm{~s}$, 6H), 1.01 (t, $J=7.3 \mathrm{~Hz}, 3 \mathrm{H}) ;{ }^{13} \mathbf{C}$ NMR (151 MHz, $\left.\mathbf{C D C l}_{3}\right): \delta 210.4,138.1,132.1,128.5$, $127.8,127.8,86.5,84.9,71.2,31.6,25.0,24.7,7.5$; the carbon bearing the boron atom could not be detected due to quadrupolar effects; ${ }^{11} \mathbf{B}$ NMR (192 $\left.\mathbf{M H z}, \mathbf{C D C l}_{3}\right): \delta 29.7$; HRMS (DART): Calcd for $\mathrm{C}_{19} \mathrm{H}_{28} \mathrm{BO}_{4}[\mathrm{M}+\mathrm{H}]^{+}: 331.2078$, Found: 331.2075 ; specific rotation: $[\alpha]_{\mathrm{D}}{ }^{20}$ $=-25.5\left(c 1.0, \mathrm{CHCl}_{3}\right)$ for an enantiomerically enriched sample of 97:3 e.r.

Enantiomeric purity was determined by HPLC analysis in comparison with authentic racemic material; $(R, R)$-Whelk-O1 column, 99:1 hexanes/i-PrOH, $0.5 \mathrm{~mL} / \mathrm{min}, 220 \mathrm{~nm}$.
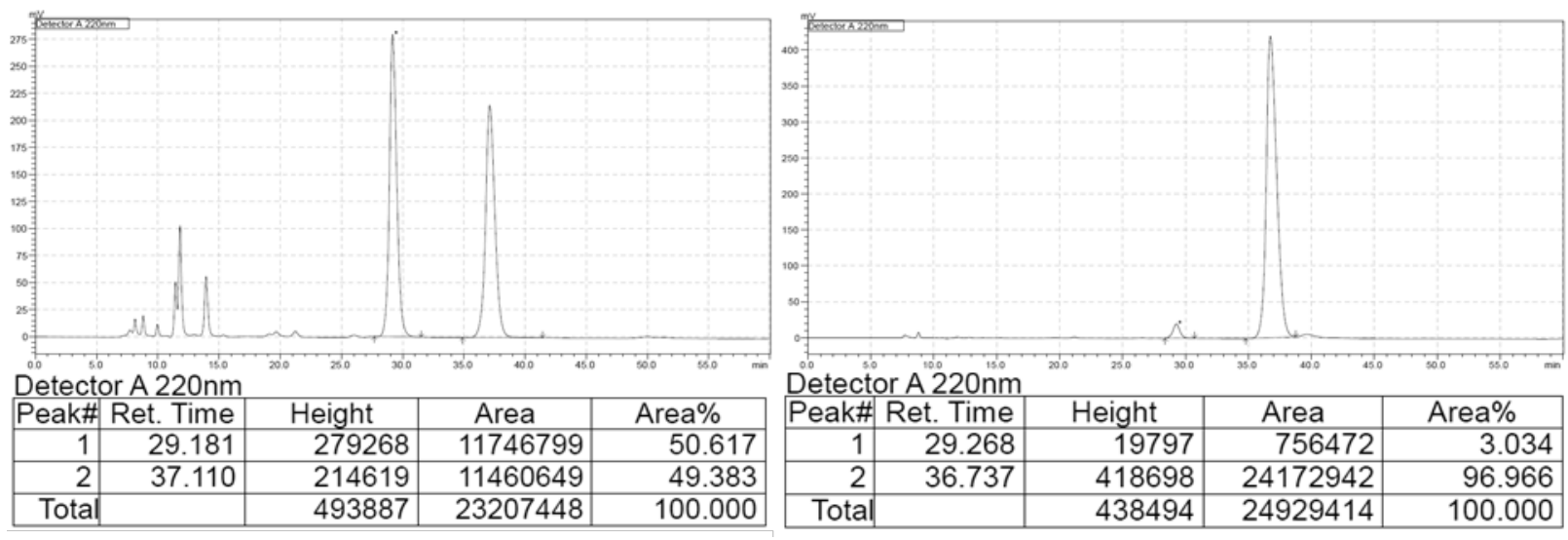

\begin{tabular}{|c|c|c|c|c|c|}
\hline Retention Time & Area & Area\% & Retention Time & Area & Area \% \\
\hline 29.181 & 279268 & 50.617 & 29.268 & 756472 & 3.034 \\
\hline 37.110 & 214619 & 49.383 & 36.737 & 24172942 & 96.966 \\
\hline
\end{tabular}

6.6.6 (R)-3-((4-Methoxybenzyl)oxy)-1-phenyl-4-(4,4,5,5-tetramethyl-1,3,2dioxaborolan-2-yl)pent-4-en-2-one (6f)<smiles>C=C(Br)C(O[Ga])C(=O)Br</smiles>

Colorless oil; $523.6 \mathrm{mg}, 1.24$ mmol, 62\% yield, 98:2 e.r.; IR (neat): 2974 (w), 2925 (w), 1721 (w), 1611 (s), 1512 (s), 1247 (s), 1140 (s) cm ${ }^{-1}$; ${ }^{1} \mathbf{H}$ NMR (600 MHz, CDCl $): \delta 7.31-7.19$ (m, $5 \mathrm{H}), 7.18-7.13(\mathrm{~m}, 2 \mathrm{H}), 6.90-6.84(\mathrm{~m}, 2 \mathrm{H}), 6.12(\mathrm{~d}, J=2.9 \mathrm{~Hz}, 1 \mathrm{H}), 6.02(\mathrm{~d}, J=2.9 \mathrm{~Hz}, 1 \mathrm{H})$, $4.53(\mathrm{~s}, 1 \mathrm{H}), 4.51(\mathrm{~d}, J=11.3 \mathrm{~Hz}, 1 \mathrm{H}), 4.34(\mathrm{~d}, J=11.3 \mathrm{~Hz}, 1 \mathrm{H}), 3.92(\mathrm{~d}, J=16.3 \mathrm{~Hz}, 1 \mathrm{H})$, $3.87(\mathrm{~d}, J=16.3 \mathrm{~Hz}, 1 \mathrm{H}), 3.81(\mathrm{~s}, 3 \mathrm{H}), 1.26$ (s, 12H). ${ }^{13} \mathbf{C}$ NMR (151 MHz, CDCl $):$ 159.4, 134.4, 132.84, $130.0129 .9,129.6,128.5,126.7,113.9,85.9,84.0,71.0,55.4,45.1,25.0$, 24.7. The carbon bearing the boron atom could not be detected due to quadrupolar effects; ${ }^{11} \mathbf{B}$ 
NMR (192 MHz, CDCl 3 ): $\delta$ 29.6 HRMS (DART): Calcd for $\mathrm{C}_{25} \mathrm{H}_{35} \mathrm{BNO}_{5}\left[\mathrm{M}+\mathrm{NH}_{4}\right]^{+}$: 440.2609, Found: 440.2608; specific rotation: $[\alpha]_{\mathrm{D}}{ }^{20}=-49.2\left(c\right.$ 1.0, $\left.\mathrm{CHCl}_{3}\right)$ for an enantiomerically enriched sample of $98: 2$ e.r.

Enantiomeric purity was determined by HPLC analysis in comparison with authentic racemic material; Chiralcel OD-H column, 95.0:5.0 hexanes $/ i-\mathrm{PrOH}, 0.5 \mathrm{~mL} / \mathrm{min}, 220 \mathrm{~nm}$.
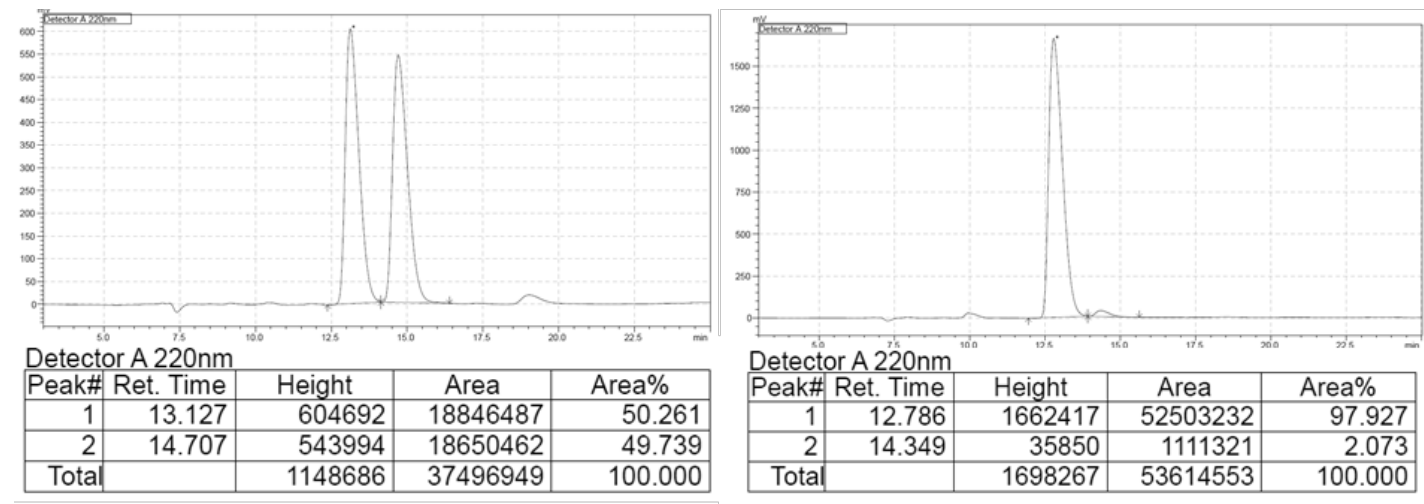

\begin{tabular}{|c|c|c|c|c|c|}
\hline Retention Time & Area & Area $\%$ & Retention Time & Area & Area $\%$ \\
\hline 13.127 & 18846487 & 50.261 & 12.768 & 52503232 & 97.927 \\
\hline 14.707 & 18650462 & 49.739 & 14.439 & 1111321 & 2.073 \\
\hline
\end{tabular}

\section{Studies Regarding Mechanism and Variations in Conditions}

\subsection{Impact of time on enantioselectivity in a reaction of a nitrile with an electron- deficient substituent}

Because of increased acidity of the proton at the stereogenic carbon center, products derived from nitrile that contain an electron-deficient moiety are especially prone to epimerization and olefin isomerization.

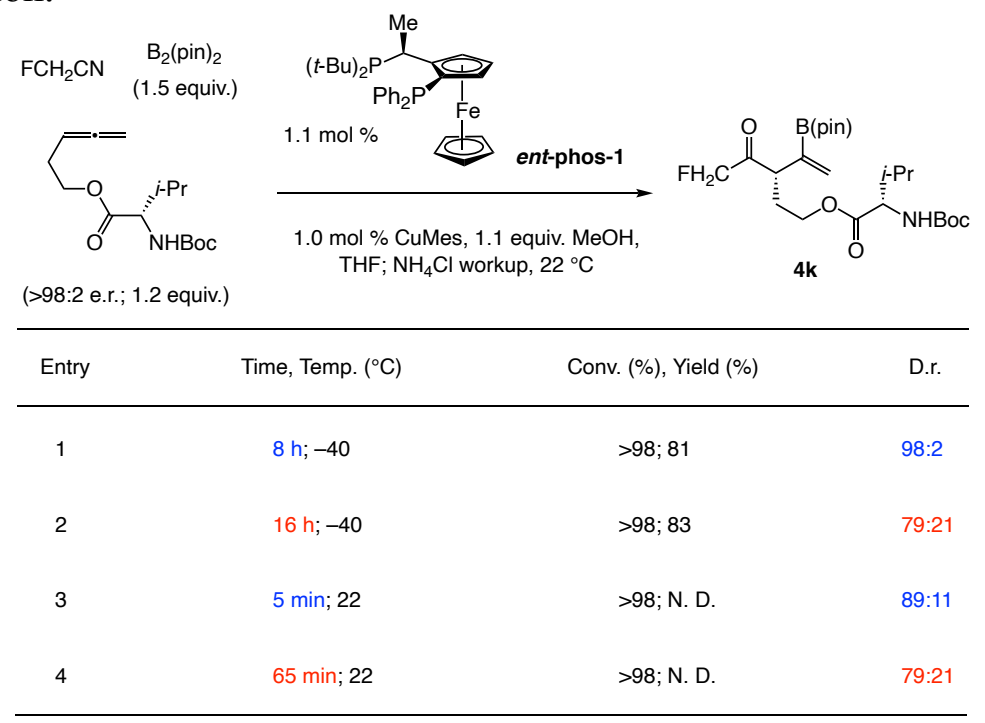

Table S1. Impact of time on e.r. in a reaction of a nitrile with an electron-deficient substituent. Reactions were performed under $\mathrm{N}_{2}$ atm. Conversion (nitrile disappearance) and diastereomeric ratio (d.r.) values were determined by analysis of ${ }^{1} \mathrm{H}$ NMR spectra of unpurified product mixtures $( \pm 2 \%)$. Yields are for isolated and purified products $( \pm 5 \%)$. pin, pinacolato; Mes, 2,4-6-trimethylphenyl; Boc, benzyloxycarbonyl; N. D., not determined. 


\subsection{Impact of alcohol size on enantioselectivity}

When a larger alcohol is used (e.g., $i-\mathrm{PrOH}$ vs $\mathrm{MeOH}$ ) transformations were less efficient and enantioselective (Table S2). This is likely because protonolysis of intermediate complex is slower, catalyst turnover is less efficient, longer reactions times are needed, allowing the N-H ketimine to undergo epimerization prior to hydrolysis to afford the ketone product (Scheme $\mathrm{S} 1)$. This is a problem that is particular to synthesis of $\beta, \gamma$-unsaturated ketones, because, in contrast to previous cases (homoallylic amine synthesis) where in situ reduction or use of low temperature addressed the epimerization/olefin isomerization problem, here, the ketimine must survive intact until the addition of water, which must be performed at ambient temperature.

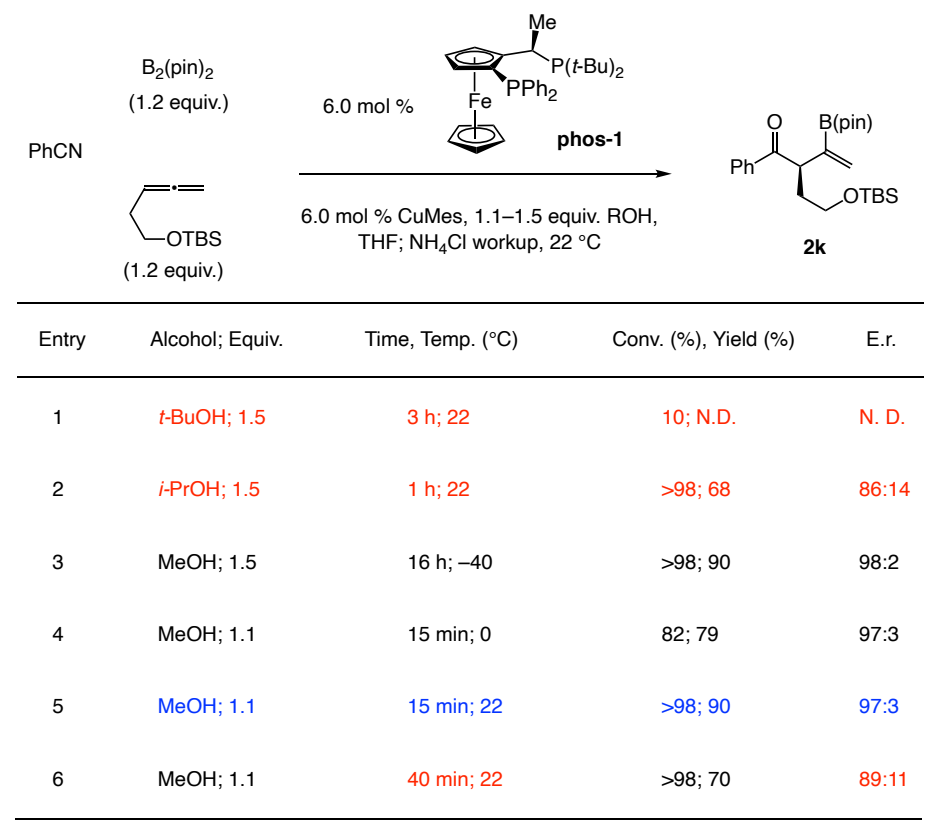

Table S2. Impact of alcohol size on yield and enantioselectivity. When a larger alcohol is used (e.g., $t$ - $\mathrm{BuOH}$ vs. $i$-PrOH vs. $\mathrm{MeOH}$ ), protonolysis of intermediate complex is slower, catalyst turnover is less efficient, longer reactions times are needed, allowing the $\mathrm{N}-\mathrm{H}$ ketimine to undergo epimerization prior to hydrolysis to afford the ketone product.

Reactions were performed under $\mathrm{N}_{2} \mathrm{~atm}$. Conversion (nitrile disappearance) was determined by analysis of ${ }^{1} \mathrm{H}$ NMR spectra of unpurified product mixtures $( \pm 2 \%)$. Yields are for isolated and purified products $( \pm 5 \%)$. Enantiomeric ratio (e.r.) values were determined by HPLC analysis $( \pm 1 \%)$. pin, pinacolato; Mes, 2,4,6-trimethylphenyl; TBS, $t$-butyldimethylsilyl; N. D., not determined.

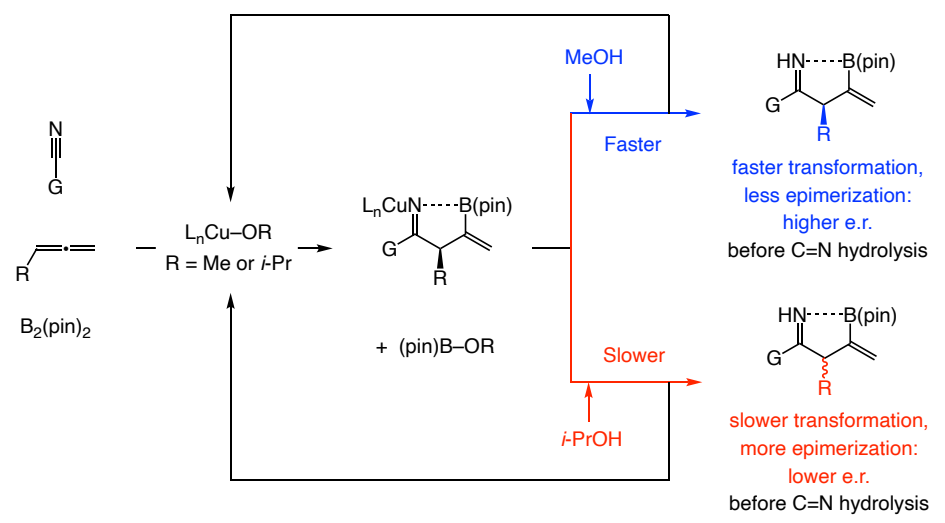

Scheme S1. The reason for reduced enantioselectivity with larger alcohols. Reactions were performed under $\mathrm{N}_{2}$ atm. Conversion (nitrile disappearance) was determined by analysis of ${ }^{1} \mathrm{H}$ NMR spectra of unpurified product mixtures $( \pm 2 \%)$. Yields are for isolated and purified products $( \pm 5 \%)$. Enantioselectivities were determined by HPLC analysis $( \pm 1 \%)$. pin, pinacolato; TBS, $t$-butyldimethylsilyl; N. D., not determined. 


\subsection{Use of a more robust $\mathrm{Cu}$ salt}

Preliminary investigations revealed that a reaction carried out with $\left[\mathrm{CuF}\left(\mathrm{PPh}_{3}\right)_{3} \cdot 2 \mathrm{EtOH}\right]$ (Table S3) was similarly efficient and enantioselective compared to when CuMes is used, despite being performed at ambient temperature, which allowed for complete conversion after just 15 min (entries 1-2). As before, extended reaction times can lead to epimerization (major) and some alkene isomerization (minor; entry 3 ).

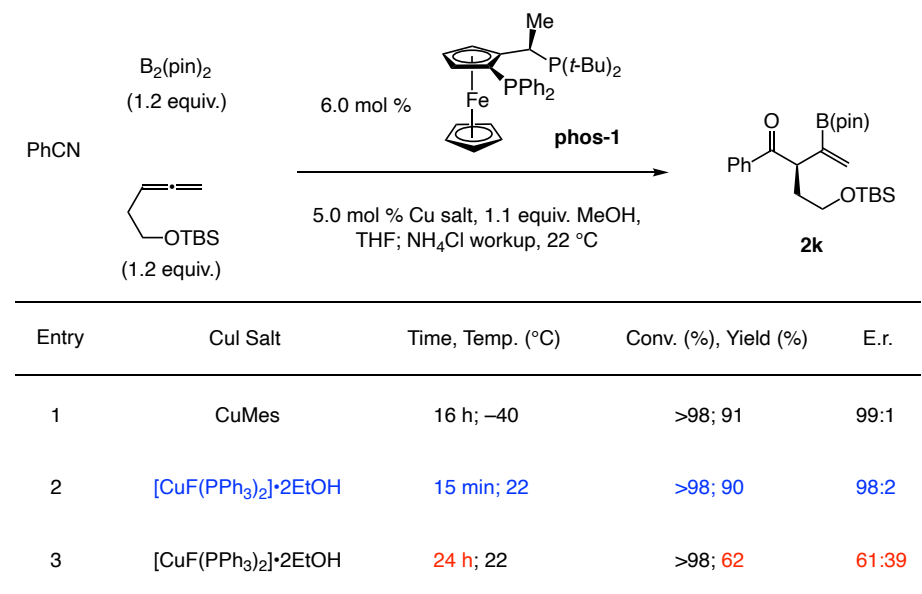

Table S3. Effect of Cu Salt on Efficiency and Enantioselectivity. Reactions were performed under $\mathrm{N}_{2}$ atm. Conversion (nitrile disappearance) determined by analysis of ${ }^{1} \mathrm{H}$ NMR spectra of unpurified product mixtures $( \pm 2 \%)$. Yields are for isolated and purified products $( \pm 5 \%)$. Enantioselectivities were determined by HPLC analysis $( \pm 1 \%)$. pin, pinacolato; TBS, $t$-butyldimethylsilyl; N. D., not determined.

\subsection{The optimal conditions for reactions involving 1,1-disubstituted allenes}

The use of a more sterically demanding chiral ligand and alcohol, namely DTBM-segphos (also needed for high e.r.) and $i-\mathrm{PrOH}$ allows for minimization of adventitious protonolysis of the intermediate $\mathrm{Cu}$-allyl compound (to give byproduct $\mathbf{A}$ ), resulting in more efficient processes (Table S4). Because epimerization is not an issue with these transformations, slower rate of catalyst $\left(\mathrm{P}_{2} \mathrm{Cu}-\mathrm{OR}\right)$ regeneration is not a concern.

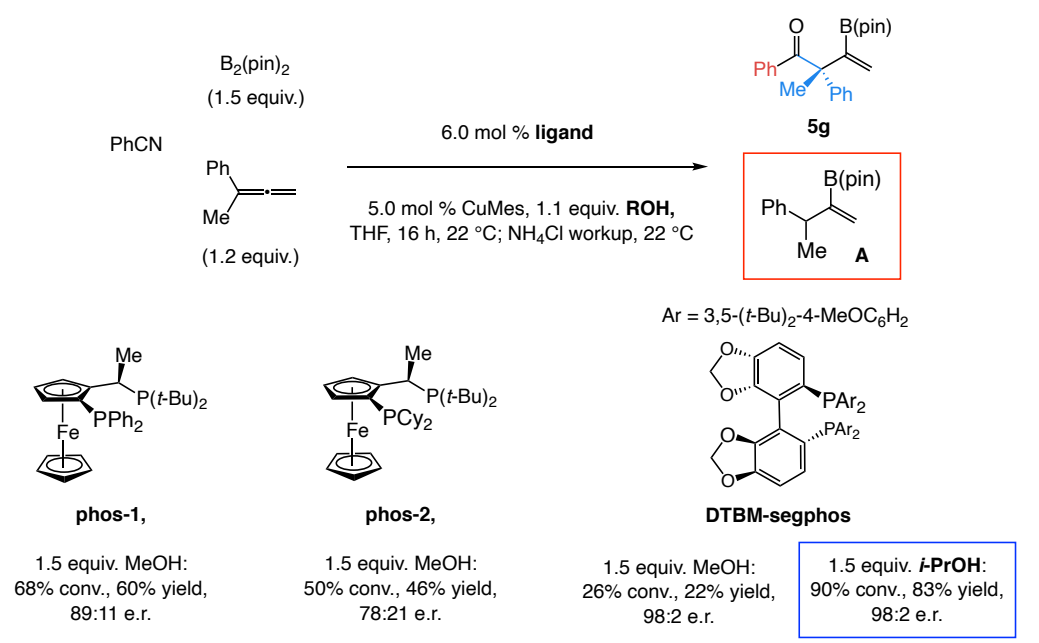

Table S4. The optimal conditions for reactions involving 1,1-disubstituted allenes. Reactions were performed under $\mathrm{N}_{2}$ atm. Conversion (nitrile disappearance) was determined by analysis of ${ }^{1} \mathrm{H}$ NMR spectra of unpurified product mixtures $( \pm 2 \%)$. Yields are for isolated and purified products $( \pm 5 \%)$. Enantiomeric ratio (e.r.) values were determined by HPLC analysis $( \pm 1 \%)$. pin, pinacolato; Mes, $2,4-6-$ trimethylphenyl. 


\subsection{The impact of ketimine hydrolysis conditions on product enantioselectivity}

A number of conditions were investigated for hydrolysis of $\beta$-boryl $\beta, \gamma$-unsaturated ketimines. In the case of $\mathbf{2 k}$, filtration of the reaction mixture through a plug of silica gel afforded the desired product in 78:22 er (Table S5, entry 1). When an aqueous solution of saturated ammonium chloride was used to quench the reaction, there was minimal epimerization (98:2 er; entry 2). However, in the case of $\mathbf{2} \mathbf{i}$, which involves the intermediacy of a ketimine bearing a more sensitive aryl-substituted stereogenic carbon center, use of the latter conditions (i.e., $\mathrm{NH}_{4} \mathrm{Cl}$ ) enantioselectiomeric purity of the product was moderate (87:13 er; entry 3 ). The same was observed with a saturated aqueous solution of $\mathrm{NaCl}$ (84:16 er; entry 4). The optimal conditions were thus determined to involve the use of a $0.5 \mathrm{M}$ aqueous solution of $\mathrm{HCl}$ at -40 ${ }^{\circ} \mathrm{C}$ (95:5 er; entry 5); this is probably owing to faster hydrolysis of the ketimine intermediate, leading to minimal epimerization.

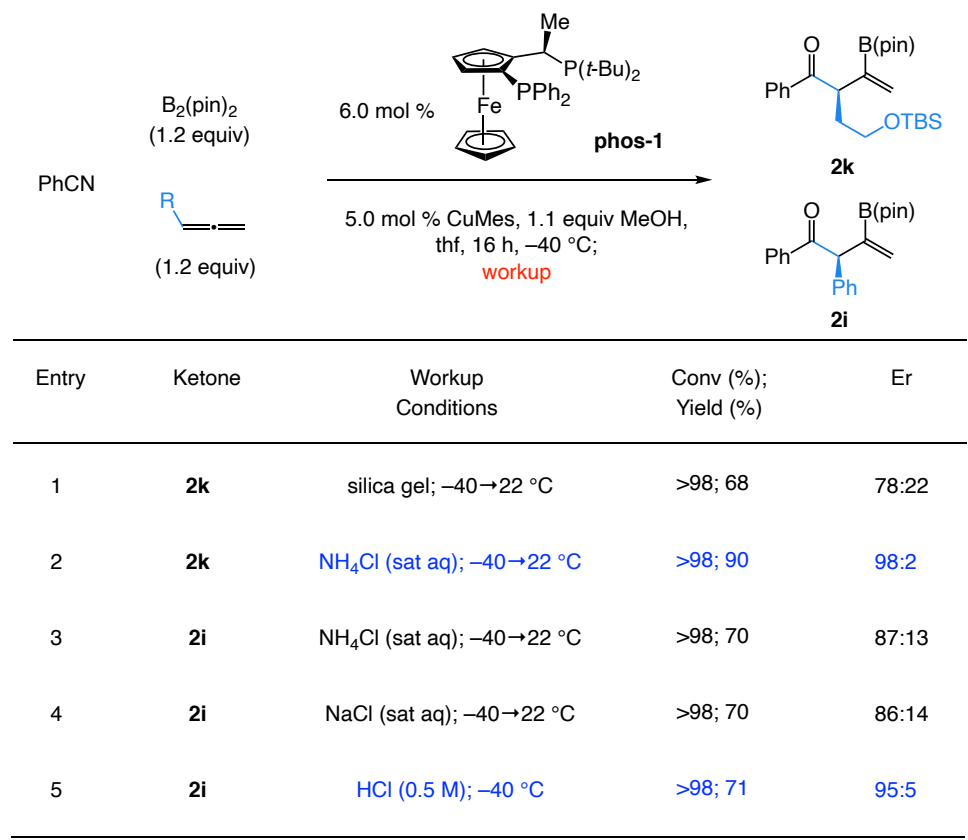

Table S5. Effect of workup conditions on product enantioselectivity. Reactions were performed under $\mathrm{N}_{2}$ atm. Conversion (nitrile disappearance) was determined by analysis of ${ }^{1} \mathrm{H}$ NMR spectra of unpurified product mixtures ( $\left.\pm 2 \%\right)$. Yields are for isolated and purified products $( \pm 5 \%)$. Enantiomeric ratio (e.r.) values were determined by HPLC analysis ( $\pm 1 \%)$. pin, pinacolato; Mes, 2,4-6-trimethylphenyl.

\section{Diastereoselective Additions to $\beta$-Boryl $\beta, \gamma$-Unsaturated Ketones}

\subsection{Identification of optimal conditions}

A number of conditions were investigated. Because of the difference in reactivity and size of different nucleophiles and difficult-to-predict impact of enthalpic and/or entropic factors, we surmised that different sets of conditions and optimal reagents would be needed, and this indeed proved to be the case.

\subsubsection{Addition of an alkyl moiety}

The majority of commonly used alkylmetal compounds, such as $\mathrm{MeMgBr}$ and $\mathrm{Me}_{2} \mathrm{Zn}$, proved to be ineffective (Table S6). Only an alkyllithium reagent proved to be sufficiently reactive, affording the desired tertiary alcohol in high yield (e.g., $(\boldsymbol{R}, \boldsymbol{R})-\mathbf{7 a}$ in 91:9 d.r., 83\% yield, 97:3 e.r., after oxidative workup). Diastereoselectivity is consistent with addition occurring through 
the Felkin-Anh-type transition state. Solvent polarity proved to be critical, with a non-polar medium being advantageous (compare entries 1-4 and 5-6), likely because entropic factors are dominant under these conditions ${ }^{28}$.

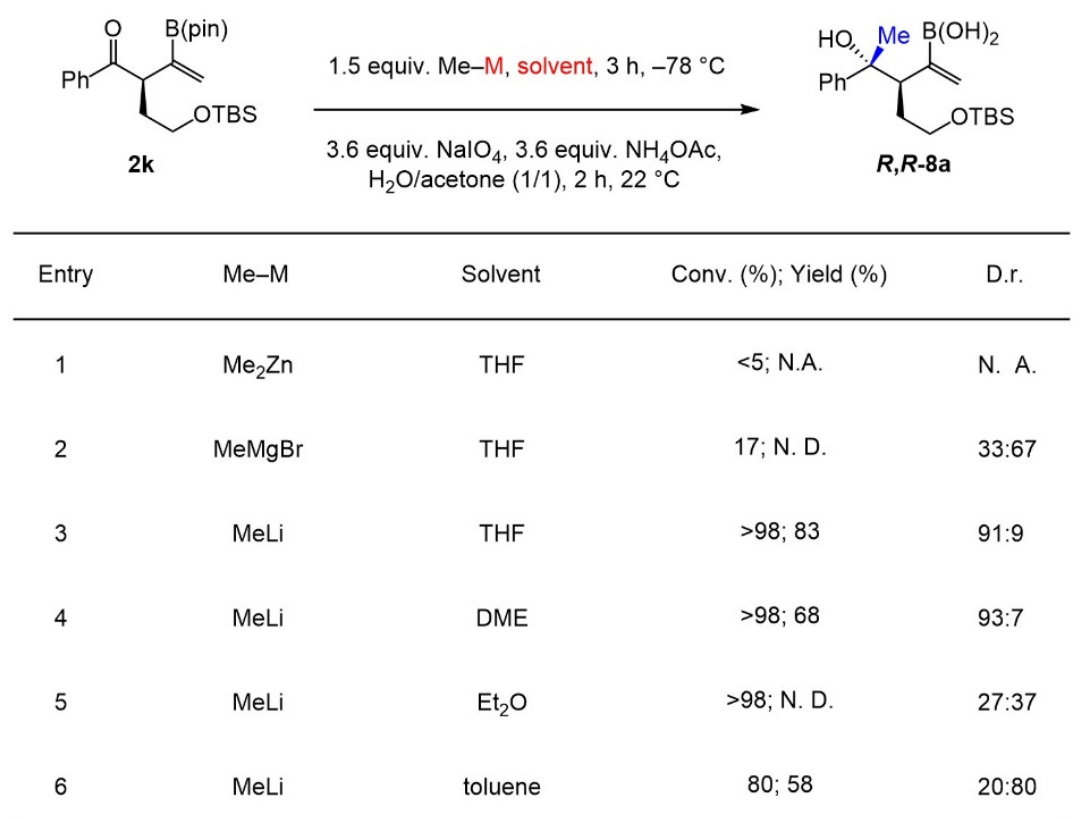

Table S6. Determining the optimal methyl metal compound, phase 1. Reactions were performed under $\mathrm{N}_{2}$ atm. Conversion (ketone disappearance) and diastereoselectivity were determined by analysis of ${ }^{1} \mathrm{H}$ NMR spectra of unpurified product mixtures ( $\pm 2 \%$ ). Yields are for isolated and purified products $( \pm 5 \%)$. pin, pinacolato; TBS, t-butyldimethylsilyl; N. A., not applicable; N. D., not determined.

Based on the reasoning that in a process largely controlled by steric factors, a more sizeable nucleophile should lead to higher diastereoselectivity, we first investigated reactions with as Ti-, Mn-, and Yb-based allylmetal compounds (Table S7, entries 1-4). However, these conditions led to inferior efficiency. In contrast, when an organocerium compound was used, additions were efficient and diastereoselective (entries 5-7), with the more soluble $\mathrm{Ce}(\mathrm{O} i \text { - } \mathrm{Pr})_{3}$ being optimal (likely owing to minimal direct MeLi addition). Accordingly, we surmised that by including $\mathrm{LiCl}$, which helps solubilize the Ce-based salt (probably by forming an anionic complex), might further facilitate transformation ${ }^{29}$. In the event, $\mathrm{CeCl}_{3} \cdot 2 \mathrm{LiCl}$ was found to be optimal (entry 7). 


\begin{tabular}{|c|c|c|c|c|}
\hline $4 r$ & DTBS & \multicolumn{2}{|c|}{$\begin{array}{c}1.5 \text { equiv. Me-M, } 2.0 \text { equiv. additive, } \\
\mathrm{THF}, 3 \mathrm{~h},-78^{\circ} \mathrm{C}\end{array}$} & 7b \\
\hline Entry & Additive & Conv. (\%) & Yield (\%) & D.r. \\
\hline 1 & None & $>98$ & 65 & $82: 18$ \\
\hline 2 & $\mathrm{ClTi}(\mathrm{O} i-\mathrm{Pr})_{3}$ & 10 & N. D. & N. D. \\
\hline 3 & $\mathrm{Mn}(\mathrm{OPiv})_{2}$ & $<5$ & N. A. & N. A. \\
\hline 4 & $\mathrm{Yb}(\mathrm{OTf})_{3}$ & 40 & 30 & $95: 5$ \\
\hline 5 & $\mathrm{CeCl}_{3}$ & $>98$ & 75 & $90: 10$ \\
\hline 6 & $\mathrm{Ce}(\mathrm{O} i-\mathrm{Pr})_{3}$ & $>98$ & 69 & $93: 7$ \\
\hline 7 & $\mathrm{CeCl}_{3} \cdot 2 \mathrm{LiCl}$ & $>98$ & 85 & $93: 7$ \\
\hline
\end{tabular}

Table S7. Determining the optimal methyl metal compound, phase 2. Reactions were performed under $\mathrm{N}_{2}$ atm. Conversion (ketone disappearance) and diastereoselectivity were determined by analysis of ${ }^{1} \mathrm{H}$ NMR spectra of unpurified product mixtures ( $\pm 2 \%$ ). Yields are for isolated and purified products $( \pm 5 \%)$. pin, pinacolato; TBS, $t$-butyldimethylsilyl; N. A., not applicable; N. D., not determined.

\subsubsection{Addition of an aryl moiety}

Solvent polarity is crucial here as well (Scheme S2). More specifically, d.r. vlues were higher with 2-Me-THF compared to DME or THF (87:13 d.r. and 91:9). Although the reasons for the diastereoselectivity changes are unclear, aggregation state changes in the organolithium reagent might be involved ${ }^{30}$, resulting in increased effectiveness of a less coordinating medium.
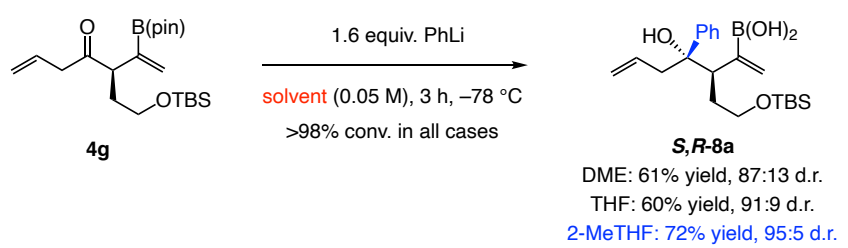

Scheme S2. Determining the optimal conditions for diastereoselective aryl additions. Reactions were performed under $\mathrm{N}_{2}$ atm. Conversion (nitrile disappearance) and diastereoselectivity were determined by analysis of ${ }^{1} \mathrm{H}$ NMR spectra of unpurified product mixtures $( \pm 2 \%)$. Yields are for isolated and purified products $( \pm 5 \%)$. pin, pinacolato; TBS, $t$-butyldimethylsilyl; N. D., not determined.

\subsubsection{Addition of an allyl moiety}

Several different allylmetal compounds were investigated, including $\mathrm{Ce}-\mathrm{Cu}-$, and Ti-based compounds, which were generated in situ by reaction of an allyl- $\mathrm{MgCl}$ or allyl- $\mathrm{ZnBr}$. Appreciable diastereoselectivity was observed in all cases (Table S8). While a blend of allyl$\mathrm{MgCl}$ and cerium trichloride in THF allowed us to convert $\mathbf{2} \mathbf{k}$ to $(R, R)-\mathbf{8 a}$ in $80 \%$ yield and 91:9 d.r. after $10 \mathrm{~min}$ at room temperature (entry 4), a more effective protocol entailed the use of manganese pivaloate as the additive ${ }^{8}$ (entry $8 ; 80 \%$ yield, $97: 3$ d.r.). The improved selectivity observed for $\mathrm{Mn}(\mathrm{OPiv})_{2}$ may be attributed to the large size of the anionic ligand. This hypothesis is supported by the observation that when $\mathrm{MnCl}_{2}$ and $\mathrm{MnBr}_{2}$ were used, d.r. values were lower (entries 6-7). 


\begin{tabular}{|c|c|c|c|c|c|}
\hline \multicolumn{3}{|c|}{ 2k } & \multicolumn{3}{|c|}{$R, R-7 \mathrm{a}$} \\
\hline Entry & Allyl-M & $\begin{array}{l}\text { Time (h); } \\
\text { Temp }\left({ }^{\circ} \mathrm{C}\right)\end{array}$ & Additive (mol \%) & $\begin{array}{l}\text { Conv. (\%); } \\
\text { Yield (\%) }\end{array}$ & D.r. \\
\hline 1 & allyl-ZnBr & $1 ; 0$ & None & $>98 ;$ N. D. & 86:14 \\
\hline 2 & allyl-ZnBr & $1 ; 0$ & $\mathrm{Cu}\left(\mathrm{PPh}_{3}\right)_{3} \mathrm{Br} ; 5$ & $>98 ; 73$ & $84: 16$ \\
\hline 3 & allyl-MgBr & $6 ;-78$ & $\mathrm{Ce}(\mathrm{O}-\mathrm{Pr})_{3} \& \mathrm{LiCl} ; 150 \& 500$ & $>98 ; 72$ & $70: 30$ \\
\hline 4 & allyl-MgBr & $6 ;-78$ & $\mathrm{CeCl}_{3} ; 150$ & $>98 ; 80$ & 91:9 \\
\hline 5 & allyl-MgBr & $1 ; 22$ & $\mathrm{Ti}(\mathrm{Ot}-\mathrm{Bu})_{4} ; 150$ & 95; N. D. & 83:17 \\
\hline 6 & allyl-MgBr & $6 ;-78$ & $\mathrm{MnCl}_{2} ; 150$ & $95 ;$ N. D. & $85: 15$ \\
\hline 7 & allyl-MgBr & $6 ;-78$ & $\mathrm{MnBr}_{2} ; 150$ & $>98 ;$ N. D. & $86: 14$ \\
\hline 8 & allyl-MgBr & $6 ;-78$ & $\mathrm{Mn}(\mathrm{OPiv})_{2} ; 150$ & $>98 ; 80$ & 97:3 \\
\hline
\end{tabular}

Table S8. Determining the optimal conditions for allyl addition. Reactions were performed under $\mathrm{N}_{2}$ atm. Conversion (ketone disappearance) and diastereoselectivity were determined by analysis of ${ }^{1} \mathrm{H}$ NMR spectra of unpurified product mixtures ( $\pm 2 \%$ ). Yields are for isolated and purified products $( \pm 5 \%)$. pin, pinacolato; TBS, $t$-butyldimethylsilyl; N. A., not applicable; N. D., not determined.

\subsubsection{Addition of an alkenyl moiety}

Addition of vinyl- $\mathrm{MgBr}$ to ketone $\mathbf{2} \mathbf{k}$ was inefficient ( $<2 \%$ conv.) and the presence of $\mathrm{Ce}(\mathrm{O} i$ $\mathrm{Pr})_{3}$ did little to improve matters (Table S9, entry 1). With LiCl efficiency was higher (entries 3-4), probably because it positively impacts $\mathrm{Mg} / \mathrm{Ce}$ exchange. After examining several other additive options (entries 4-5) we were able to establish that the combination of 2:1 mixture of $\mathrm{LiCl}$ and $\mathrm{CeCl}_{3}$ are optimal (entry 6). These findings are key because Grignard reagents are more functional group tolerant and easier to handle than their organolithium counterparts. For example, whereas vinyl- $\mathrm{MgBr}$ is commercially available, vinyl-Li must be prepared through somewhat tedious procedures involving Sn-based reagents ${ }^{31}$.

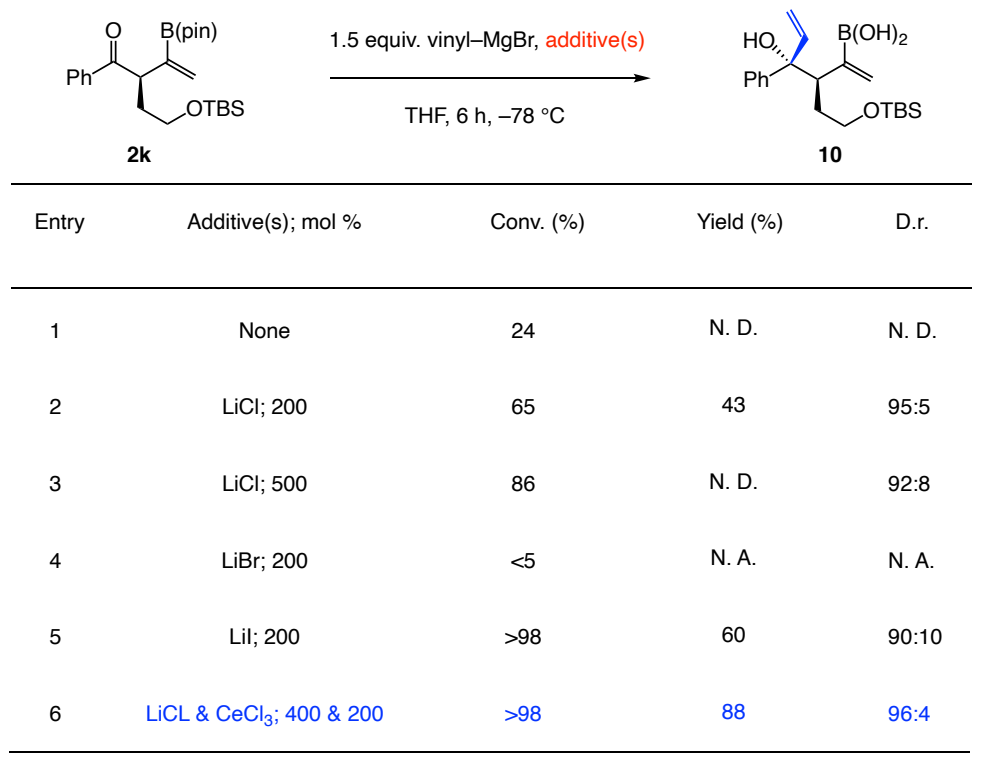


Table S9. Determining the optimal conditions for alkenyl addition. Reactions were performed under $\mathrm{N}_{2}$ atm. Conversion (ketone disappearance) and diastereoselectivity were determined by analysis of ${ }^{1} \mathrm{H}$ NMR spectra of unpurified product mixtures ( $\pm 2 \%$ ). Yields are for isolated and purified products ( $\pm 5 \%$ ). pin, pinacolato; TBS, $t$-butyldimethylsilyl; N. A., not applicable; N. D., not determined.

\subsubsection{Regarding boronic acid products}

Isolation of alkenyl-B(pin) products is not a simple task because the $\beta$-hydroxy group causes partial removal of the pinacolato moiety (Scheme S3). Different work-up procedures were developed to address this issue, allowing us to isolate the derived boronic acids easily and in high yield (i.e., hydrolysis/oxidation with $\mathrm{NaIO}_{4}$ and oxidation with $\mathrm{NaBO}_{3} \bullet 4 \mathrm{H}_{2} \mathrm{O}$; see below for experimental details).

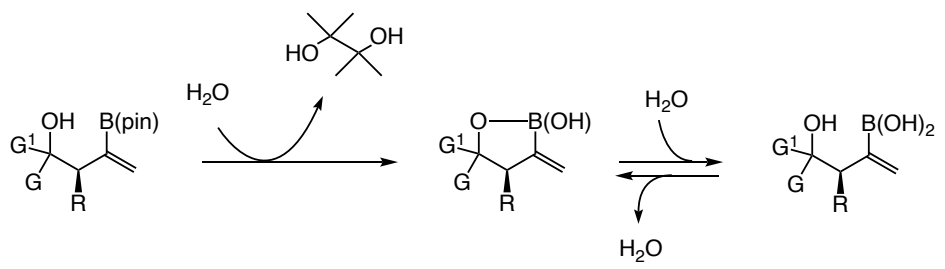

Scheme S3. $\beta$-Hydroxy-assisted hydrolysis of a B(pin) moiety.

\subsection{Procedures for Addition of an Organometallic Compounds to a $\beta, \gamma$ - Unsaturated Ketone}

\subsubsection{General procedure for addition of an organometallic compound based on $\mathrm{CeCl}_{3} \cdot 2 \mathrm{LiCl}$}

In a $\mathrm{N}_{2}$-filled glove box, an oven-dried vial $(4 \mathrm{~mL}, 17 \times 38 \mathrm{~mm})$ containing a stir bar was charged with $\mathrm{CeCl}_{3}$ (49.3 mg, $0.20 \mathrm{mmol}, 2.0$ equiv.), $\mathrm{LiCl}$ (17.0 mg, $0.40 \mathrm{mmol}, 4.0$ equiv.), and THF $(1.0 \mathrm{~mL})$. The vial was capped with a septum and the suspension was allowed to stir for $1 \mathrm{~h}$. The vial was removed from the glove box and the mixture was subjected to sonication for $5 \mathrm{~min}$, at which time a milky suspension was formed. The solution was then allowed to cool to $-78^{\circ} \mathrm{C}$ and kept at this temperature for $30 \mathrm{~min}$ (vessel was connected to a $\mathrm{N}_{2}$-filled Schlenk line placed in a dry ice/acetone bath). A solution of the organometallic compound (1.8 equiv.) was added dropwise by syringe, after which the mixture was allowed to stir at $-78^{\circ} \mathrm{C}$ for $1 \mathrm{~h}$. A solution of the ketone (1.0 equiv.; e.g., $0.10 \mathrm{mmol}$ in $0.50 \mathrm{~mL}$ THF) was added dropwise by syringe, and the resulting mixture was allowed to stir for $2.5 \mathrm{~h}$ at $-78{ }^{\circ} \mathrm{C}$, and then at room temperature for $10 \mathrm{~min}$. At this time, the reaction was quenched by the addition of a saturated aqueous solution of sodium and potassium tartrate and the solution was allowed to stir vigorously for $30 \mathrm{~min}$ at ambient temperature. The solution was washed with $\mathrm{Et}_{2} \mathrm{O}(10 \mathrm{~mL} \times$ 3 ) and the volatiles were removed in vacuo to afford the unpurified tertiary alcohol, which was subjected to one of the work-up procedures detailed below.

\subsubsection{For addition of an organolithium compound (no additive)}

In a $\mathrm{N}_{2}$-filled glove box, an oven-dried vial ( $4 \mathrm{~mL}, 17 \times 38 \mathrm{~mm}$ ) equipped with a stir bar was charged with a ketone $(0.10 \mathrm{mmol}, 1.0$ equiv. $)$ and 2-Me-THF $(2.0 \mathrm{~mL})$. The vial was sealed (screw cap and PTFE septum) and was removed from the glove box. The mixture was then allowed to cool to $-78{ }^{\circ} \mathrm{C}$ and stay at this temperature for $15 \mathrm{~min}$, (vessel was connected to a $\mathrm{N}_{2}$-filled Schlenk line placed in a dry ice/acetone bath). A solution of the organolithium $(0.12$ 
mmol, 1.2 equiv.) was added dropwise through an oven-dried syringe $\left(-78^{\circ} \mathrm{C}\right)$, after which the mixture was allowed to stir for $3 \mathrm{~h}$ at $-78{ }^{\circ} \mathrm{C}$. The reaction mixture was allowed to warm to room temperature and the reaction was quenched by the addition of a saturated aqueous solution of $\mathrm{NH}_{4} \mathrm{Cl}(1.0 \mathrm{~mL})$. After more of the $\mathrm{NH}_{4} \mathrm{Cl}$ solution was added $(5.0 \mathrm{~mL})$, the mixture was washed with $\mathrm{Et}_{2} \mathrm{O}(2 \times 3.0 \mathrm{~mL})$ and then $\mathrm{CH}_{2} \mathrm{Cl}_{2}(3.0 \mathrm{~mL})$. The organic layers were combined and the volatile were removed in vacuo to afford a residue, which was subjected to a workup procedure, as detailed below (see section 8.3).

\subsubsection{For addition of a Grignard reagent to an $\alpha$-substituted OPMB ketone}

In a $\mathrm{N}_{2}$-filled glove box, an oven-dried vial ( $4 \mathrm{~mL}, 17 \times 38 \mathrm{~mm}$ ) equipped with a stir bar was charged with a ketone $(0.10 \mathrm{mmol}, 1.0$ equiv. $)$ and THF $(1 \mathrm{~mL})$. The vial was sealed (screw cap and PTFE septum) and was removed from the glove box. The solution was allowed to cool to $-78^{\circ} \mathrm{C}$ and stir for $30 \mathrm{~min}$, (vessel was connected to a $\mathrm{N}_{2}$-filled Schlenk line placed in a dry ice/acetone bath). A solution of $\mathrm{MeMgBr}$ (in $\mathrm{Et}_{2} \mathrm{O}, 0.30 \mathrm{mmol}, 3.0$ equiv.) was added dropwise by syringe, and the mixture was allowed to stir at $-78{ }^{\circ} \mathrm{C}$ for $1 \mathrm{~h}$, and then at room temperature for $10 \mathrm{~min}$. At this time, the reaction was quenched by the addition of a saturated solution of aqueous sodium-potassium tartrate $(1 \mathrm{~mL})$, after which the solution was allowed to stir vigorously for $30 \mathrm{~min}$ at room temperature. The mixture was then washed with $\mathrm{Et}_{2} \mathrm{O}(3 \times 10$ $\mathrm{mL}$ ) and the volatiles were removed under reduced pressure to afford a residue, which was subjected to a workup procedure, as detailed below (see section 8.3).

\subsubsection{For addition of allyl-MgCl in combination with $\mathrm{Mn}(\mathrm{OPiv})_{2}$}

The procedure was adapted from a previously reported protocol. ${ }^{8}$ In a $\mathrm{N}_{2}$-filled glove box, an oven-dried vial ( $4 \mathrm{~mL}, 17 \times 38 \mathrm{~mm})$ equipped with a stir bar was charged with manganese(II) pivalate $(0.20 \mathrm{mmol}, 2.0$ equiv. $)$ and THF $(1 \mathrm{~mL})$. The vial was sealed (screw cap and PTFE septum) and the solution was allowed to stir until it became homogeneous. The vial was removed from the glove box and the vessel was connected to a $\mathrm{N}_{2}$-filled Schlenk line placed in a dry ice/acetone bath for $15 \mathrm{~min}$. The mixture was allowed to cool to $-78{ }^{\circ} \mathrm{C}$ and a solution of allyl- $\mathrm{MgCl}$ (0.22 M in THF, $0.15 \mathrm{mmol}, 1.5$ equiv.) was added dropwise by oven-dried syringe over $2 \mathrm{~min}$, causing the light pink solution to turn dark red; stirring was allowed to continue for $35 \mathrm{~min}$ at $-78^{\circ} \mathrm{C}$. The ketone $(0.10 \mathrm{mmol})$, dissolved in THF $(1.0 \mathrm{~mL})$, and added dropwise to the mixture at $-78{ }^{\circ} \mathrm{C}$ in a pan of $3 \mathrm{~min}$. The mixture allowed to stir for $1 \mathrm{~h}$ at $-78^{\circ} \mathrm{C}$ after which the reaction was quenched by addition of a saturated solution of $\mathrm{NaHCO}_{3}(1.0 \mathrm{~mL})$ at low temperature. The mixture was allowed to warm to room temperature, diluted by addition of more of the $\mathrm{NaHCO}_{3}$ solution $(10.0 \mathrm{~mL})$, and washed with $\mathrm{Et}_{2} \mathrm{O}(2 \times 3.0 \mathrm{~mL})$ and $\mathrm{CH}_{2} \mathrm{Cl}_{2}$ $(3.0 \mathrm{~mL})$. The organic layers were combined and the volatiles were removed in vacuo to afford a residue that was subjected to a work-up procedure detailed below (see section 8.3).

\subsubsection{For addition of an allylmagnesium halide compound to an $\alpha$-substituted OPMB ketone}

The procedure was adapted from a previously reported protocol.$^{32}$ In a $\mathrm{N}_{2}$-filled glove box, an oven-dried vial $\left(4 \mathrm{~mL}, 17 \times 38 \mathrm{~mm}\right.$ ) was equipped with a solution of allyl- $\mathrm{MgBr}$ (in $\mathrm{Et}_{2} \mathrm{O}, 0.20$ 
mmol, 2.0 equiv.). The solvent was removed in vacuo and dried under high vacuum (0.8 mbar) for $10 \mathrm{~min}$, and then $\mathrm{CH}_{2} \mathrm{Cl}_{2}(0.4 \mathrm{~mL})$ was added. The vial was capped with a septum, removed from the glovebox, and the solution subjected to sonication for $5 \mathrm{~min}$, leading to generation of a milky off-white suspension. A second oven-dried vial with a stir bar was charged with ketone 6f $(0.10 \mathrm{mmol}, 1.0$ equiv. $)$ and $\mathrm{CH}_{2} \mathrm{Cl}_{2}(1 \mathrm{~mL})$. The vial containing the ketone was removed from the glovebox, the mixture was allowed to cool to $-78{ }^{\circ} \mathrm{C}$ (vessel was connected to a $\mathrm{N}_{2}$ filled Schlenk line placed in a dry ice/acetone bath), after which it was charged with the solution of allyl- $\mathrm{MgBr}$ in $\mathrm{CH}_{2} \mathrm{Cl}_{2}$ was added dropwise by syringe. The mixture was allowed to stir at $78^{\circ} \mathrm{C}$ for $1 \mathrm{~h}$, and then at room temperature for $10 \mathrm{~min}$. At this time, the reaction was quenched by the addition of a saturated aqueous solution of $\mathrm{NaHCO}_{3}(1 \mathrm{~mL})$ and the mixture was to stir vigorously for $30 \mathrm{~min}$. The mixture was washed with $\mathrm{Et}_{2} \mathrm{O}(3 \times 10 \mathrm{~mL})$ and the volatiles were removed in vacuo, affording a residue that was subjected to a work-up procedure detailed below (see section 8.3).

\subsubsection{For addition of a propargylzinc compound}

A representative procedure is as follows: 3-Bromo-1-trimethylsilyl-1-propyne $(4.86 \mathrm{~mL}, 30.0$ mmol) was added to a suspension of activated $\mathrm{Zn}$ powder $(5.88 \mathrm{~g}, 90.0 \mathrm{mmol})$ in THF $(25 \mathrm{~mL})$. The mixture was slowed to heat to $55{ }^{\circ} \mathrm{C}$ for $15 \mathrm{~min}$, resulting in the formation of $\mathrm{Me}_{3} \mathrm{Si}$ substituted propargyl-ZnBr. The solution was allowed to cool to $22{ }^{\circ} \mathrm{C}$, and then was added (1.0 M solution in THF, $26.2 \mathrm{~mL}, 26.2 \mathrm{mmol}$ ) was added at $0{ }^{\circ} \mathrm{C}$ to a solution of ketone $4 \mathbf{d}$ $(4.11 \mathrm{~g}, 13.1 \mathrm{mmol})$ in THF (50 mL, vessel was connected to a $\mathrm{N}_{2}$-filled Schlenk line placed in an ice/water bath). The mixture was allowed to stir for $2 \mathrm{~h}$ after which it was allowed to warm to $22{ }^{\circ} \mathrm{C}$. It was then diluted with an aqueous solution of $\mathrm{pH}=7$ buffer (di-sodium hydrogen phosphate/potassium-dihydrogen phosphate, $70 \mathrm{~mL})$ and $\mathrm{NaBO}_{3} \bullet 4 \mathrm{H}_{2} \mathrm{O}(6.05 \mathrm{~g}, 39.3$ mmol). The resulting mixture was allowed to stir vigorously at $22^{\circ} \mathrm{C}$ for $1 \mathrm{~h}$; it was then diluted with $\mathrm{Et}_{2} \mathrm{O}(50 \mathrm{~mL})$, the phases were separated, and the aqueous phase was washed with $\mathrm{Et}_{2} \mathrm{O}$ $(3 \times 50 \mathrm{~mL})$. The combined organic layers were dried over $\mathrm{MgSO}_{4}$, filtered and concentrated under reduced pressure, affording a yellowish oil residue, which was purified by silica gel chromatography $\left(9: 1 \rightarrow 4: 1\right.$ hexanes: $\mathrm{Et}_{2} \mathrm{O} ; \mathrm{R}_{f}=0.21$ in 4:1 hexanes: $\left.\mathrm{Et}_{2} \mathrm{O}\right)$ afforded lactone $\mathbf{1 6 b}$ as a colorless oil ( $4.20 \mathrm{~g}, 10.2 \mathrm{mmol}, 78 \%$ yield, $>98: 2$ d.r.).

\subsection{The Workup Procedures}

\subsubsection{Leading to Boronic Acid Products with $\mathrm{NaBO}_{3} \bullet 4 \mathrm{H}_{2} \mathrm{O}$}

Unpurified $\beta$-boryl tertiary alcohol was dissolved in a 1:1 THF/water and the solution was transferred to a vial containing a magnetic stir bar and $\mathrm{NaBO}_{3} \bullet 4 \mathrm{H}_{2} \mathrm{O}$ (5.0 equiv.) and a magnetic stir bar. The mixture was allowed to stir at ambient temperature while being monitored by TLC. Once full conversion was detected (typically, 20-30 min), the solution was washed three times with $\mathrm{Et}_{2} \mathrm{O}$ and the combined organic layers were dried over $\mathrm{MgSO}_{4}$. Filtration and removal of volatiles in vacuo afforded a residue (often light-yellow oil), which was purified by chromatography (see note below for recommended silica gel treatment). 


\subsubsection{Leading to $\beta$-Hydroxy Ketone Products with $\mathrm{NaIO}_{4}$}

Unpurified $\beta$-boryl tertiary alcohol was dissolved in a 1:1 acetone/water $(2.0 \mathrm{~mL})$ and the solution was transferred to a vial containing a magnetic stir bar and $\mathrm{NaIO}_{4}$ (3.5 equiv.), and $\mathrm{NH}_{4} \mathrm{OAc}$ (3.0 equiv.). The mixture was allowed to stir for $2.5 \mathrm{~h}$ at room temperature and was then washed three times with $\mathrm{Et}_{2} \mathrm{O}$. The combined organic layers were dried over $\mathrm{MgSO}_{4}$. Filtration and removal of volatiles in vacuo afforded a residue (often light-yellow oil), which was purified by chromatography.

The following points merit note:

1) Diastereomeric purity was typically determined by ${ }^{1} \mathrm{H}$ NMR in $\mathrm{CDCl}_{3}$ with $\sim 50 \mu \mathrm{L}$ $\mathrm{D}_{2} \mathrm{O}$.

2) $\beta$-Hydroxy boronic acid products can be sensitive to oxidative work-up and can be converted to $\beta$-hydroxy ketone. It is therefore recommended that reaction progress is monitored to circumvent this issue, which can lead to lower yields.

3) $\beta$-Hydroxy ketones are prone decomposition through retro-aldol processes. This decomposition is exacerbated by silica gel and can be minimized if the silica gel used has been kept in in an open container in a $150{ }^{\circ} \mathrm{C}$ oven for at least three days.

\subsection{Regarding possible loss of enantiomeric purity upon nucleophilic addition to an $\alpha$-substituted ketone}

The enantiomeric purity of a number of different tertiary alcohols, derived from the addition of organometallic nucleophiles to enantioenriched $\alpha$-substituted $\beta$-boryl ketones, were analyzed by HPLC in order to determine whether the stereochemical purity of the starting ketone was preserved (Scheme S4). Thus, reactions of various $\alpha$-substituted ketones with alkyl-, allyl-, aryl-, and alkynyl-metal compounds ( $\mathrm{Li}-, \mathrm{Mg}-$, Ce-, and $\mathrm{Mn}$-based) were probed. In none of the cases investigated was there any loss of enantiomeric purity detected.
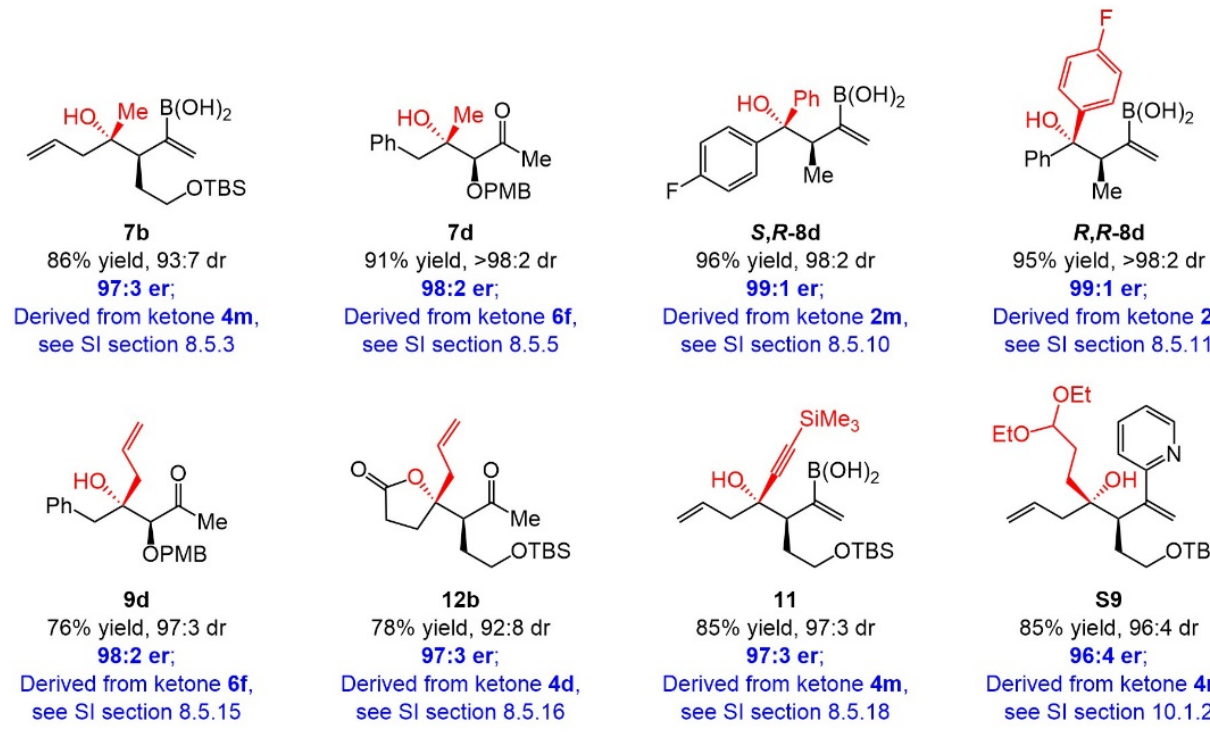

$$
\begin{gathered}
\text { 95\% yield, >98:2 dr } \\
\text { 99:1 er; } \\
\text { Derived from ketone 2I, }
\end{gathered}
$$

see SI section 8.5.11
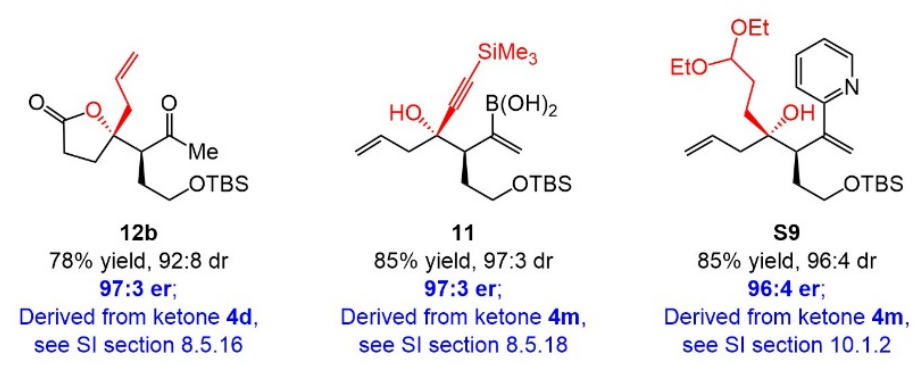

Scheme S4. Enantiomeric purity of Tertiary alcohols. In none of the cases investigated was there any loss of enantiomeric purity observed, compared to the a-substituted ketone substrate. 


\subsection{Analytical Data for Tertiary Alcohols}

\subsection{1 ((3R,4R)-3-(2-((t-Butyldimethylsilyl)oxy)ethyl)-4-hydroxy-4-phenylpent-1-en-2-} yl)boronic acid $[(R, R)-7 a]$<smiles>CC(=O)OCCC(C(=O)O)C(O)(O)c1ccccc1</smiles>

Prepared according to the procedure described in section 8.2.1 with commercially available $\mathrm{MeMgCl}$ (3.0 M in THF), followed by $\mathrm{NaIO}_{4}$ workup (2 h).

Colorless oil; $28.1 \mathrm{mg}, 0.0771 \mathrm{mmol}, 77 \%$ yield, 97:3 d.r.; IR (neat): 3375 (br), 2951 (m), 2926 (m), 2881 (m), 1444 (s), 1403 (s), 1294 (m), 1376 (m), 1254 (s), 1096 (s), 1067 (m), 936 (w), 834 (s), 761 (s), 667 (s) cm ${ }^{-1}$; ${ }^{1} \mathbf{H}$ NMR (600 MHz, CDCl $): \delta 7.47-7.42$ (m, 2H), 7.34$7.32(\mathrm{~m}, 2 \mathrm{H}), 7.28-7.22(\mathrm{~m}, 1 \mathrm{H}), 5.73(\mathrm{~s}, 1 \mathrm{H}), 5.57$ (s, 1H), 3.66 (ddd, $J=10.1,7.5,4.8 \mathrm{~Hz}$, 1H), 3.59 (ddd, $J=10.1,7.9,6.6 \mathrm{~Hz}, 1 \mathrm{H}), 2.99-2.92$ (m, 1H), 1.93-1.84 (m, 1H), 1.77-1.68 (m, 1H), $1.49(\mathrm{~s}, 3 \mathrm{H}), 0.84(\mathrm{~s}, 10 \mathrm{H}),-0.01(\mathrm{~s}, 3 \mathrm{H}),-0.02(\mathrm{~s}, 3 \mathrm{H}) ;{ }^{13} \mathbf{C}$ NMR (151 MHz, CDCl $\left._{3}\right): \delta 147.1,146.5$ (C-B bs), 128.3, 127.2, 125.1, 122.2, 86.2, 61.3, 53.0, 32.6, 26.0, 24.5, 18.4, -5.21, -5.24; ${ }^{11} \mathbf{B}$ NMR (160 $\left.\mathbf{~ M H z , ~} \mathbf{C D C l}_{3}\right): \delta$ 31.6; HRMS (DART): Calcd for $\mathrm{C}_{19} \mathrm{H}_{32} \mathrm{BO}_{3} \mathrm{Si}\left[\mathrm{M}+\mathrm{H}-\mathrm{H}_{2} \mathrm{O}\right]^{+}: 347.2208$, Found: 347.2210 ; specific rotation: $[\alpha]_{\mathrm{D}}{ }^{20}=+6.5(c$ $1.0, \mathrm{CHCl}_{3}$ ) for an enantiomerically enriched sample of 99:1 e.r., which is the enantiomeric purity of the starting ketone (2k).

\subsection{2 ((3R,4S)-3-(2-((t-Butyldimethylsilyl)oxy)ethyl)-4-hydroxy-4-phenylpent-1-en-2-} yl)boronic acid $[(S, R)-7 \mathrm{a}]$

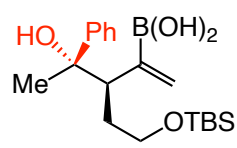

Prepared according to the procedure described in section 8.2.2 with commercially available $\mathrm{PhLi}\left[1.78 \mathrm{M}\right.$ in $\left.(n-\mathrm{Bu})_{2} \mathrm{O}\right]$, followed by $\mathrm{NaIO}_{4}$ workup $(2 \mathrm{~h})$.

Colorless oil; 29.5 mg, 0.081 mmol, 81\% yield, 92:8 d.r.; IR (neat): 3351 (br), 2951 (m), 2926 (m), 2854 (m), 1494 (m), 1444 (m), 1400 (m), 1275 (m), 1255 (m), 1092 (s), 1064 (m), 835 (s), $774(\mathrm{~m}), 700(\mathrm{~m}) \mathrm{cm}^{-1}$; ${ }^{1} \mathbf{H}$ NMR (600 MHz, $\left.\mathbf{C D C l}_{3}\right): \delta 7.36-7.28(\mathrm{~m}, 4 \mathrm{H}), 7.26-7.22(\mathrm{~m}, 1 \mathrm{H})$, $5.81(\mathrm{~s}, 1 \mathrm{H}), 5.64(\mathrm{~s}, 1 \mathrm{H}), 3.45(\mathrm{dd}, J=7.8,5.0 \mathrm{~Hz}, 2 \mathrm{H}), 2.94-2.83(\mathrm{~m}, 1 \mathrm{H}), 1.62(\mathrm{~s}, 3 \mathrm{H}), 1.22-$ $1.16(\mathrm{~m}, 1 \mathrm{H}), 0.97-0.87(\mathrm{~m}, 2 \mathrm{H}), 0.85(\mathrm{~s}, 9 \mathrm{H}),-0.05(\mathrm{~s}, 3 \mathrm{H}),-0.05(\mathrm{~s}, 3 \mathrm{H}) ;{ }^{13} \mathbf{C}$ NMR (151 MHz, $\left.\mathbf{C D C l}_{3}\right): \delta 144.0,128.2,126.8,125.5,123.7,86.5,60.0,52.8,33.8,31.1,26.1,18.4$, $5.16,-5.20$. The carbon bearing the boron atom could not be detected due to quadrupolar effects; ${ }^{11} \mathbf{B}$ NMR (160 MHz, CDCl $\mathbf{~}_{3}$ ): $\delta$ 31.9; HRMS (DART): Calcd for $\mathrm{C}_{19} \mathrm{H}_{32} \mathrm{BO}_{3} \mathrm{Si}$ $\left[\mathrm{M}+\mathrm{H}-\mathrm{H}_{2} \mathrm{O}\right]^{+}: 347.2208$, Found: 347.2205 ; specific rotation: $[\alpha]_{\mathrm{D}}^{20}=-22.7\left(c 1.0, \mathrm{CHCl}_{3}\right)$ for an enantiomerically enriched sample of 99:1 e.r., which is the enantiomeric purity of ketone $(4 \mathrm{~g})$. 


\subsection{3 ((3R,4S)-3-(2-((t-Butyldimethylsilyl)oxy)ethyl)-4-hydroxy-4-methylhepta-1,6-}

\section{dien-2-yl)boronic acid (7b)}

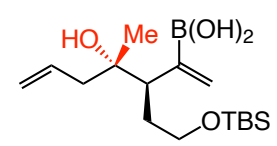

Prepared according to the procedure described in section 8.2.1 with commercially available MeLi (3.1 M in diethoxymethane) was used; $\mathrm{NaIO}_{4}$ workup (2 h).

Colorless oil; 28.1 mg, 0.0855 mmol, 86\% yield, 93:7 d.r.; IR (neat): 3367 (br), 3056 (m),2924 (s), 2853 (m), 1641 (w), 1460 (m), 1404 (m), 1253 (m), 1099 (s), 834 (s), 774 (m) cm ${ }^{-1}$; ${ }^{1} \mathbf{H}$ NMR (600 MHz, $\left.\mathbf{C D C l}_{3}+\mathbf{D}_{2} \mathbf{O}\right): \delta 5.93-5.76(\mathrm{~m}, 1 \mathrm{H}), 5.73(\mathrm{~s}, 1 \mathrm{H}), 5.58(\mathrm{~s}, 1 \mathrm{H}), 5.33(\mathrm{bs}$, $3 \mathrm{H}), 5.18-5.02(\mathrm{~m}, 2 \mathrm{H}), 3.79-3.61(\mathrm{~m}, 2 \mathrm{H}), 2.71-2.56(\mathrm{~m}, 1 \mathrm{H}), 2.40-2.26(\mathrm{~m}, 2 \mathrm{H}), 1.69-1.57$ (m, 2H), 1.12 (s, 3H), 0.90 (s, 9H), 0.05 (s, 6H); ${ }^{13} \mathbf{C}$ NMR (151 MHz, CDCl $): 147.1$ (C-B bs), $\delta 133.8,122.2,118.6,85.2,61.5,48.8,46.4,32.6,29.9,26.1,23.0,-5.1 ;{ }^{11}$ B NMR (128 MHz, CDCl 3 ): 30.1; HRMS (ESI): Calcd for $\mathrm{C}_{16} \mathrm{H}_{32} \mathrm{BO}_{3} \mathrm{Si}\left[\mathrm{M}+\mathrm{H}-\mathrm{H}_{2} \mathrm{O}\right]^{+}:$: 11.2208 , Found: 311.2211; specific rotation: $[\alpha]_{\mathrm{D}}{ }^{20}=+9.6\left(c\right.$ 1.0, $\left.\mathrm{CHCl}_{3}\right)$ for an enantiomerically enriched sample of 97:3 e.r., which corresponds to the enantiomeric purity of the ketone starting material. The e.r. for $\mathbf{7 b}$ was determined by HPLC analysis in comparison with authentic racemic material. Since the presence of a boronic acid group was found to be complicate for HPLC analysis (significant peak broadening), the alkenyl boronate was converted to the pyridinyl derivative S1, obtained by catalytic cross-coupling with 2-bromopyridine. Chiralcel AZ-H column, 95:5 hexanes/i-PrOH, $0.3 \mathrm{~mL} / \mathrm{min}, 220 \mathrm{~nm}$.

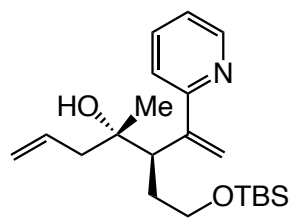

S1
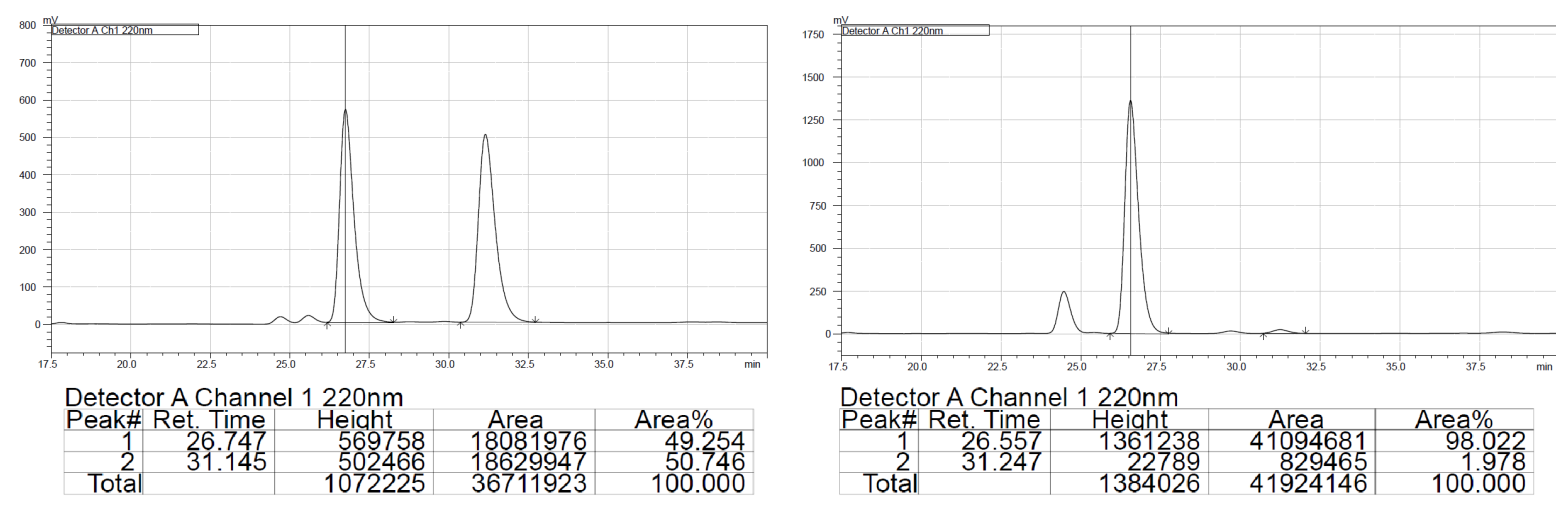

\begin{tabular}{|c|c|c|c|c|c|}
\hline Retention Time & Area & Area\% & Retention Time & Area & Area\% \\
\hline 26.747 & 18081976 & 49.254 & 26.557 & 41094681 & 98.022 \\
\hline 31.145 & 18629947 & 50.746 & 31.247 & 829465 & 1.978 \\
\hline
\end{tabular}




\subsection{4 ((3R,4S)-3-(2-((t-Butyldimethylsilyl)oxy)ethyl)-4-hydroxy-4,5,5-trimethylhex-1-} en-2-yl)boronic acid (7c)

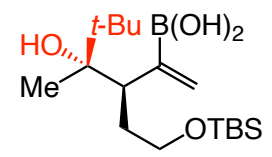

Synthesized according to the procedure described in section 8.2.2 with commercially available $t$-BuLi (1.6 $\mathrm{M}$ in pentane), followed by $\mathrm{NaIO}_{4}$ workup (3 h).

Pale-yellow oil; $20.9 \mathrm{mg}, 0.061 \mathrm{mmol}, 61 \%$ yield, $97: 3$ d.r. determined by ${ }^{1} \mathrm{H} \mathrm{NMR}$ in $\mathrm{CDCl}_{3}$ with a drop of $\mathrm{D}_{2} \mathrm{O}$; IR (neat): 3368 (br), 2951 (m), 2926 (m), 1459 (s), 1444 (s), 1403 (s), 1255 (s), 1097 (s), 1068 (m), 835 (s), 775 (s), 698 (s) cm ${ }^{-1} ;{ }^{1} \mathbf{H}$ NMR (400 MHz, CDCl $)$ : $\delta$ $5.54(\mathrm{~d}, J=3.0,1 \mathrm{H}), 5.41(\mathrm{~d}, J=3.0,1 \mathrm{H}), 3.84-3.75(\mathrm{~m}, 1 \mathrm{H}), 3.69-3.59(\mathrm{~m}, 1 \mathrm{H}), 2.95-2.64$ (m, 1H), 2.13-2.01 (m, 1H), 1.95-1.82 (m, 1H), $1.36(\mathrm{~s}, 3 \mathrm{H}), 0.94(\mathrm{~s}, 9 \mathrm{H}), 0.89(\mathrm{~s}, 9 \mathrm{H}), 0.05$ (s, 3H), 0.05 (s, 3H); ${ }^{13} \mathbf{C}$ NMR (101 MHz, $\left.\mathbf{C D C l}_{3}\right): \delta 117.5,89.4,62.2,54.7,39.8,32.7,27.2$, $26.1,25.1,18.4,-5.1,-5.2$. The carbon bearing the boron atom could not be detected due to quadrupolar effects; ${ }^{11} \mathrm{~B}$ NMR (160 $\left.\mathbf{M H z}, \mathbf{C D C l}_{3}\right): \delta$ 31.2; HRMS (DART): Calcd for $\mathrm{C}_{17} \mathrm{H}_{36} \mathrm{BO}_{3} \mathrm{Si}\left[\mathrm{M}+\mathrm{H}-\mathrm{H}_{2} \mathrm{O}\right]^{+}: 327.2521$, Found: 327.2523 ; specific rotation: $[\alpha]_{\mathrm{D}}{ }^{20}=-4.0(c$ $1.0, \mathrm{CHCl}_{3}$ ) for an enantiomerically enriched sample of 99:1 e.r., which is the enantiomeric purity of the ketone $(\mathbf{4 g})$.

\subsection{5 (3S,4S)-4-Hydroxy-3-((4-methoxybenzyl)oxy)-4-methyl-5-phenylpentan-2-one} (7d)

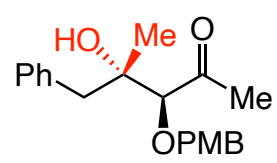

Synthesized according to the procedure described in section 8.2.3 with commercially available $\mathrm{MeMgBr}$ (in $\mathrm{Et}_{2} \mathrm{O}, 0.30 \mathrm{mmol}, 3.0$ equiv.), followed by $\mathrm{NaBO}_{3} \cdot 4 \mathrm{H}_{2} \mathrm{O}$ workup ( 30 minutes).

Colorless oil; $44.8 \mathrm{mg}, 0.1365 \mathrm{mmol}, 91 \%$ yield, >98:2 d.r., 98:2 e.r.; IR (neat): 3503 (bs), 2922 (w), 1703 (s), 1510 (s), 1246 (s) cm ${ }^{-1} ;{ }^{1} \mathbf{H}$ NMR (600 MHz, CDCl 3 ): $\delta$ 7.32-7.21 (m, $7 \mathrm{H}), 6.94-6.89(\mathrm{~m}, 2 \mathrm{H}), 4.54$ (d, $J=11.1 \mathrm{~Hz}, 1 \mathrm{H}), 4.36$ (d, $J=11.1 \mathrm{~Hz}, 1 \mathrm{H}), 3.83(\mathrm{~s}, 3 \mathrm{H}), 3.60$ (s, 1H), 2.87 (d, $J=13.6 \mathrm{~Hz}, 1 \mathrm{H}), 2.72$ (d, $J=13.6 \mathrm{~Hz}, 1 \mathrm{H}), 2.44$ (s, 1H), 2.28 (s, 3H), 1.15 (s, $3 \mathrm{H}) ;{ }^{13} \mathbf{C}$ NMR (151 MHz, $\left.\mathbf{C D C l}_{3}\right): \delta 212.9,159.7,136.7,131.0,129.9,129.2,128.2,126.7$, 114.1, 88.9, 74.4, 73.3, 55.4, 44.6, 28.7, 23.6; HRMS (DART): Calcd for $\mathrm{C}_{20} \mathrm{H}_{28} \mathrm{NO}_{4}$ $\left[\mathrm{M}+\mathrm{NH}_{4}\right]^{+}: 346.2013$, Found: 346.2017 ; specific rotation: $[\alpha]_{\mathrm{D}}{ }^{20}-46.0\left(\mathrm{c} 0.2, \mathrm{CHCl}_{3}\right)$ for an enantiomerically enriched sample of 98:2 e.r.

Enantiomeric purity of 7d was determined by HPLC analysis in comparison with authentic racemic material; $(R, R)$-Whelk-O1 column, 95.0:5.0 hexanes $/ i-\mathrm{PrOH}, 0.5 \mathrm{~mL} / \mathrm{min}, 220 \mathrm{~nm}$. This indicated no loss of enantiomeric purity (compared to ketone). 


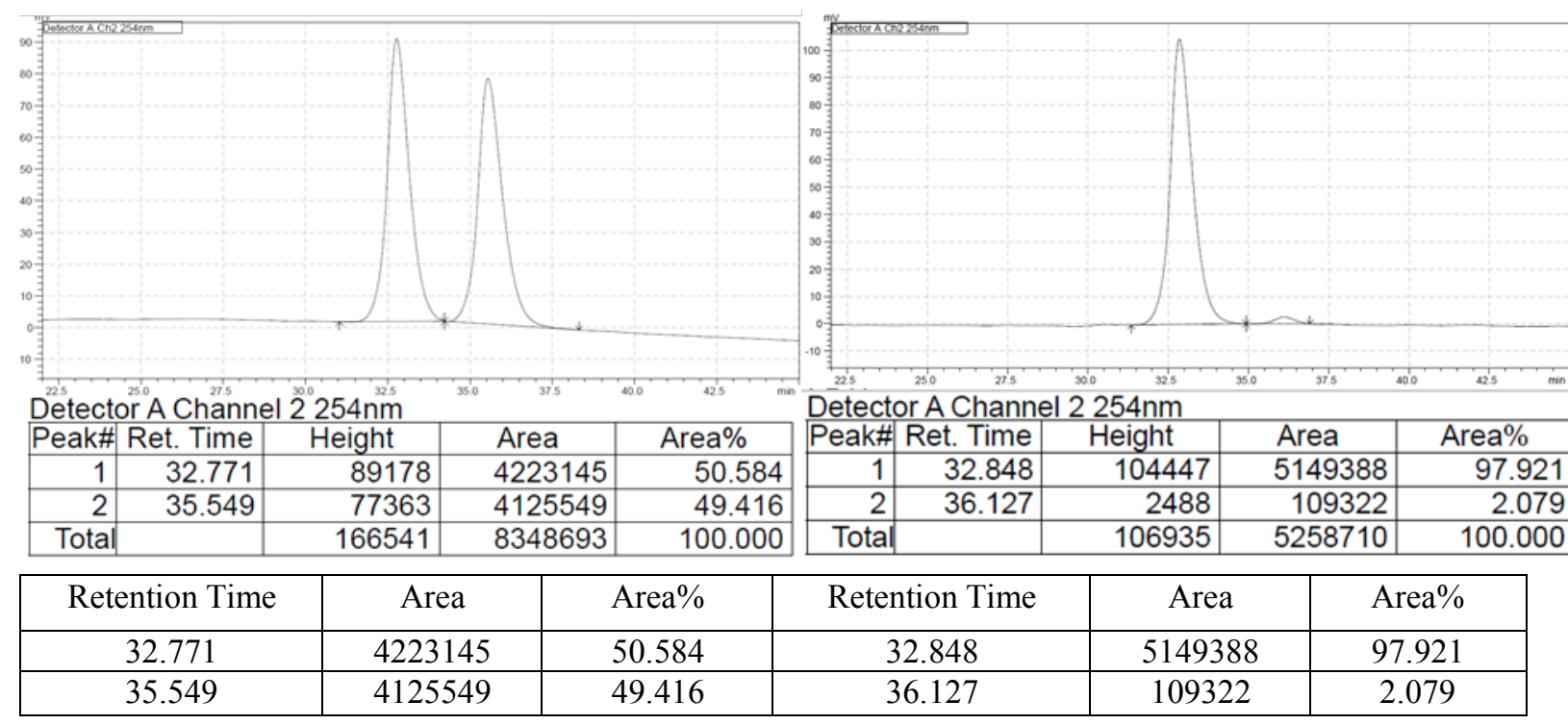

8.5.6 ((3R,4S)-3-(2-((t-butyldimethylsilyl)oxy)ethyl)-4-hydroxy-4-phenylhepta-1,6dien-2-yl)boronic acid $[(S, R)-8 \mathrm{a}]$<smiles>C=CCC(O)(CC[18OH])C(CC)C(=O)O</smiles>

Synthesized according to the procedure described in section 8.2.2 with commercially available $\mathrm{PhLi}\left[1.78 \mathrm{M}\right.$ in $\left.(n-\mathrm{Bu})_{2} \mathrm{O}\right]$, followed by $\mathrm{NaIO}_{4}$ workup $(2 \mathrm{~h})$.

Colorless oil; $28.1 \mathrm{mg}, 0.020$ mmol, 72\% yield, 95:5 d.r.; IR (neat): 3387 (br), 3060 (w), 2952 (m), 2926 (s), 2854 (m), 1642 (w), 1444 (m), 1401 (s), 1256 (s), 1098 (s), 835 (s), 774 (m) $\mathrm{cm}^{-1} ;{ }^{1} \mathbf{H}$ NMR (500 MHz, $\left.\mathbf{C D C l}_{3}\right): \delta 7.44-7.13(\mathrm{~m}, 5 \mathrm{H}), 5.82(\mathrm{~s}, 1 \mathrm{H}), 5.64(\mathrm{~s}, 1 \mathrm{H}), 5.64-5.49$ (m, 1H), 5.08-4.89 (m, 2H), 3.54-3.32 (m, 2H), 3.03-2.92 (m, 1H), $2.74(\mathrm{dd}, \mathrm{J}=14.4,6.7 \mathrm{~Hz}$, 1H), $2.61(\mathrm{dd}, \mathrm{J}=14.4,7.2 \mathrm{~Hz}, 1 \mathrm{H}), 0.85(\mathrm{~s}, 9 \mathrm{H}),-0.05$ (s, 6H) ${ }^{\mathbf{1 3}} \mathbf{C}$ NMR (126 MHz, CDCl $\left.\mathbf{3}\right)$ : $\delta 146.3$ (C-B bs), 142.1, 133.6, 128.1, 126.8, 126.0, 124.0, 118.3, 88.3, 59.7, 51.7, 48.1, 33.6, 26.1, 18.4, -5.15, -5.20. ${ }^{11} \mathbf{B}$ NMR (160 $\left.\mathbf{~ M H z , ~} \mathbf{C D C l}_{3}\right): \delta 30.8$; HRMS (DART): Calcd for $\mathrm{C}_{21} \mathrm{H}_{34} \mathrm{BO}_{3} \mathrm{Si}\left[\mathrm{M}+\mathrm{H}-\mathrm{H}_{2} \mathrm{O}\right]^{+}: 373.2365$, Found: 373.2374 ; specific rotation: $[\alpha]_{\mathrm{D}}{ }^{20}=-32.6(c$ 1.0, $\mathrm{CHCl}_{3}$ ) for an enantiomerically enriched sample of 98:2 e.r.

\subsection{7 ((3R,4R)-3-(2-((t-Butyldimethylsilyl)oxy)ethyl)-4-hydroxy-4-phenylhepta-1,6-} dien-2-yl)boronic acid $[(R, R)-8 a]$<smiles>C=CCC(O)(COCCO)C(CCO)C(O)c1ccccc1</smiles>

Synthesized according to the procedure described in section 8.2.4 with freshly prepared solution of allyl- $\mathrm{MgCl}\left(0.22 \mathrm{M}\right.$ in THF, $0.15 \mathrm{mmol}, 1.5$ equiv.), followed by $\mathrm{NaIO}_{4}$ workup (2 h).

Colorless oil; $32.0 \mathrm{mg}, 0.0820$ mmol, 82\% yield, 96:4 d.r.; IR (neat): 3377 (br), 3068 (w), 2932 (s), 2857 (m), 1446 (s), 1403 (s), 1260 (s), 1096 (s), 1005 (m), 931 (m), 836 (s), 774 (m), $702(\mathrm{~m}) \mathrm{cm}^{-1}$; ${ }^{1} \mathbf{H}$ NMR (500 MHz, $\left.\mathbf{C D C l}_{3}\right): \delta 7.43-7.36(\mathrm{~m}, 2 \mathrm{H}), 7.34-7.30(\mathrm{~m}, 2 \mathrm{H}), 7.25-$ 
$7.21(\mathrm{~m}, 1 \mathrm{H}), 5.69(\mathrm{dd}, J=2.8,2.0 \mathrm{~Hz}, 1 \mathrm{H}), 5.66-5.52(\mathrm{~m}, 2 \mathrm{H}), 5.05-4.93(\mathrm{~m}, 2 \mathrm{H}), 3.77-3.67$ (m, 1H), 3.65-3.54 (m, 1H), 2.93-2.83 (m, 1H), 2.77-2.69 (m, 1H), 2.58-2.48 (m, 1H), 2.09$1.97(\mathrm{~m}, 1 \mathrm{H}), 1.80-1.70(\mathrm{~m}, 1 \mathrm{H}), 0.83(\mathrm{~s}, 9 \mathrm{H}),-0.01$ (s, 6H); ${ }^{13} \mathbf{C} \mathbf{N M R}\left(126 \mathbf{~ M H z}, \mathbf{C D C l}_{3}\right): \delta$ 145.6, 133.6, 128.3, 127.0, 125.4, 121.8, 118.3, 87.5, 61.5, 53.9, 41.8, 32.4, 26.0, 18.4, -5.19, -5.23. The carbon bearing the boron atom could not be detected due to quadrupolar effects; ${ }^{11}$ B NMR (160 MHz, CDCl $)$ ): $\delta 31.8$; HRMS (DART): Calcd for $\mathrm{C}_{21} \mathrm{H}_{34} \mathrm{BO}_{3} \mathrm{Si}\left[\mathrm{M}+\mathrm{H}-\mathrm{H}_{2} \mathrm{O}\right]^{+}$: 373.2365, Found: 373.2357 ; specific rotation: $[\alpha]_{\mathrm{D}}{ }^{20}=+14.7$ (c 1.0, $\mathrm{CHCl}_{3}$ ) for an enantiomerically enriched sample of 98:2 e.r., which is the enantiomeric purity of the ketone (2k).

We determined the enantiomeric purity of $(\boldsymbol{R}, \boldsymbol{R})$-8a by HPLC analysis of the pyridyl derivative, S2. The presence of a boronic acid group complicates HPLC analysis (significant peak broadening). Chiralcel AZ-H column, 99:1 hexanes $/ i-\mathrm{PrOH}, 0.8 \mathrm{~mL} / \mathrm{min}, 254 \mathrm{~nm}$.

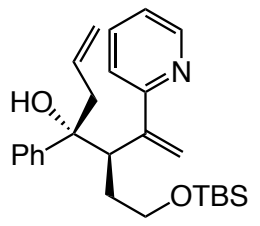

S2
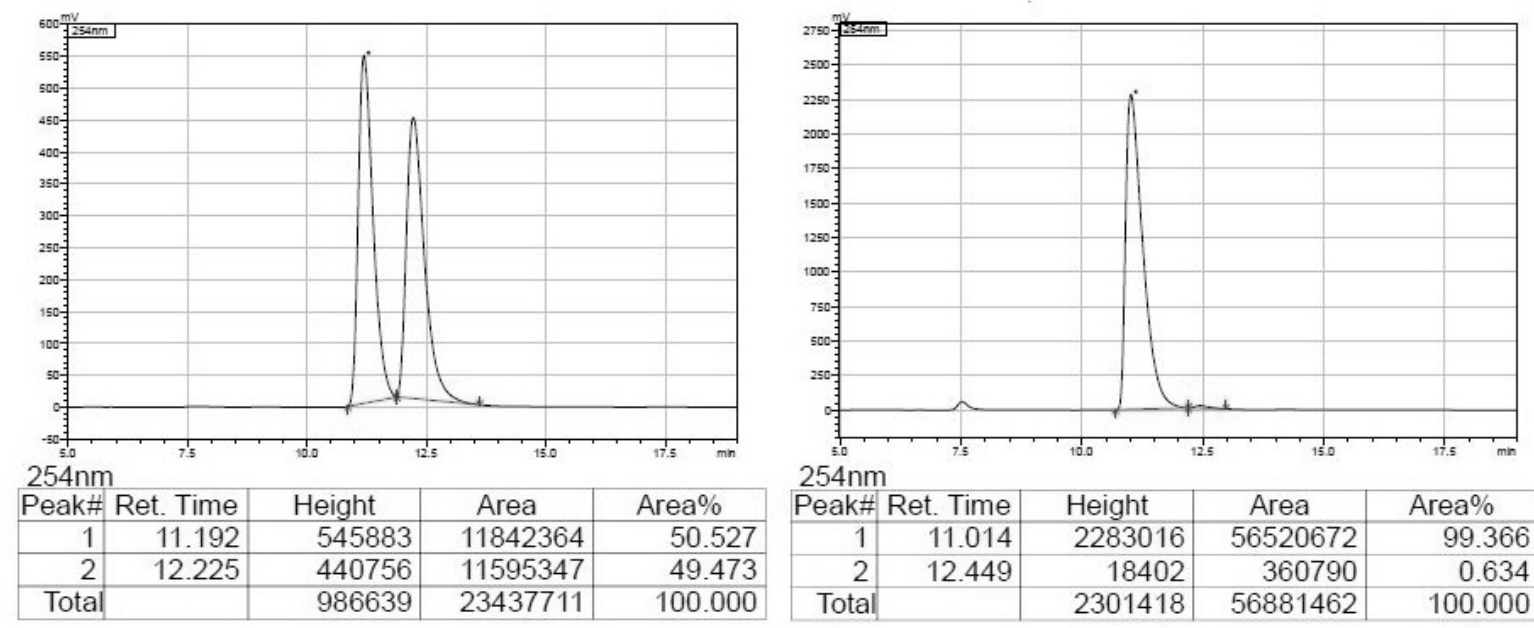

\begin{tabular}{|c|c|c|c|c|c|}
\hline Retention Time & Area & Area\% & Retention Time & Area & Area\% \\
\hline 11.192 & 11842364 & 50.527 & 11.014 & 56520672 & 99.366 \\
\hline 12.225 & 11595347 & 49.473 & 12.449 & 360790 & 0.634 \\
\hline
\end{tabular}

8.5.8 (3S,4S)-3-(2-((t-Butyldimethylsilyl)oxy)ethyl)-4-hydroxy-4-(pyridin-2-yl)pentan2-one (8b)

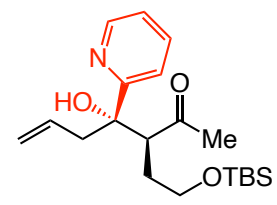

A freshly prepared solution of 2-pyridyl-Li solution $(0.5 \mathrm{~mL}, 0.36 \mathrm{M}, 0.18 \mathrm{mmol}$, prepared at $-78{ }^{\circ} \mathrm{C}$ in THF) was used. The reaction was carried out with $0.3 \mathrm{mmol} \mathbf{4 m}$, and the amounts of other reagents was adjusted accordingly. Oxidation to the $\beta$-hydroxy ketone $\left(\mathrm{NaBO}_{3} \bullet 4 \mathrm{H}_{2} \mathrm{O}\right)$ 
was necessary for efficient product isolation because the boric acid could not be obtained in high purity.

Colorless oil; $63.8 \mathrm{mg}, 0.1890$ mmol, 63\% yield, 92:8 d.r.; IR (neat): 3493 (br), 2951 (m), 2926 (m), 1696 (m), 1253 (m), 1098 (s), 833 (s), 776 (m) cm ${ }^{-1}$; ${ }^{\mathbf{H}} \mathbf{H}$ NMR (600 MHz, CDCl $)$ : $\delta 8.68-8.46(\mathrm{~m}, 1 \mathrm{H}), 7.76-7.66(\mathrm{~m}, 1 \mathrm{H}), 7.53-7.43(\mathrm{~m}, 1 \mathrm{H}), 7.18(\mathrm{~s}, 0 \mathrm{H}), 5.00(\mathrm{~s}, 1 \mathrm{H}), 3.46-$ $3.31(\mathrm{~m}, 2 \mathrm{H}), 3.22(\mathrm{dd}, J=10.4,3.1 \mathrm{~Hz}, 1 \mathrm{H}), 2.23(\mathrm{~s}, 3 \mathrm{H}), 1.92-1.83(\mathrm{~m}, 1 \mathrm{H}), 1.49(\mathrm{~s}, 3 \mathrm{H})$, $1.38-1.27(\mathrm{~m}, 1 \mathrm{H}), 0.86(\mathrm{~s}, 1 \mathrm{H}), 0.81(\mathrm{~s}, 9 \mathrm{H}),-0.07$ (s, 6H); ${ }^{13} \mathbf{C}$ NMR (151 MHz, CDCl 3$): \delta$ 214.7, 163.9, 148.0, 137.0, 122.2, 120.0, 75.4, 61.8, 59.1, 32.4, 31.5, 27.6, 26.0, 18.4, -5.41, -5.43; HRMS (ESI): Calcd for $\mathrm{C}_{18} \mathrm{H}_{32} \mathrm{NO}_{3} \mathrm{Si}[\mathrm{M}+\mathrm{H}]^{+}:$338.2146, Found: 338.2151; specific rotation: $[\alpha]_{\mathrm{D}}{ }^{20}=+13.1\left(c 0.6, \mathrm{CHCl}_{3}\right)$ for an enantiomerically enriched sample of 99:1 e.r., which is identical to the e.r. of the starting ketone $(\mathbf{4 m})$.

\subsection{9 (3S,4R)-4-hydroxy-3-((4-methoxybenzyl)oxy)-5-phenyl-4-(thiophen-3-yl)pentan-} 2-one $(8 c)$

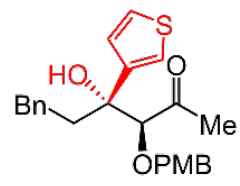

Synthesized according to the procedure described in section 8.2.3 with prepared solution of thiophen-3-ylmagnesium chloride, lithium chloride complex (in THF, $0.76 \mathrm{mmol}, 2.0$ equiv.) prepared according to a reported procedure, ${ }^{33}$ followed by $\mathrm{NaBO}_{3} \cdot 4 \mathrm{H}_{2} \mathrm{O}$ workup ( 30 minutes). Colorless oil, $47.6 \mathrm{mg}, 0.1210 \mathrm{mmol}, 80 \%$ yield, 99:1 d.r., IR (neat): 3504 (bs), 2921 (w), 1704 (s), 1611 (s), 1510 (s), 1351 (s), 1246 (s) cm ${ }^{-1}$; ${ }^{1} \mathbf{H}$ NMR (600 MHz, CDCl $): \delta$ 7.30$7.23(\mathrm{~m}, 3 \mathrm{H}), 7.22-7.18(\mathrm{~m}, 3 \mathrm{H}), 7.18-7.14(\mathrm{~m}, 1 \mathrm{H}), 7.11-7.05(\mathrm{~m}, 3 \mathrm{H}), 6.95-6.90(\mathrm{~m}, 2 \mathrm{H})$, $4.49(\mathrm{~d}, J=11.0 \mathrm{~Hz}, 1 \mathrm{H}), 4.34(\mathrm{~d}, J=11.0 \mathrm{~Hz}, 1 \mathrm{H}), 3.84(\mathrm{~s}, 3 \mathrm{H}), 3.75(\mathrm{~s}, 1 \mathrm{H}), 3.42(\mathrm{~s}, 1 \mathrm{H})$, 3.16 (s, 2H), 1.88 (s, 3H); ${ }^{13} \mathbf{C}$ NMR (151 MHz, CDCl $): \delta 214.4,159.7,144.9,136.0,131.0$, 129.6, 129.1, 128.0, 126.8, 126.6, 125.5, 122.0, 114.2, 88.9, 77.4, 73.67, 55.5, 45.8, 27.5; HRMS (DART): Calcd for $\mathrm{C}_{23} \mathrm{H}_{23} \mathrm{O}_{3} \mathrm{~S}$ [M+H- $\left.\mathrm{H}_{2} \mathrm{O}\right]^{+}:$379.1362, Found: 379.1363; Specific rotation: $[\alpha]_{\mathrm{D}}{ }^{20}=-21.2\left(c 0.2, \mathrm{CHCl}_{3}\right)$ for an enantiomerically enriched sample of 98:2 e.r.

\subsubsection{0 ((3R,4S)-4-(4-Fluorophenyl)-4-hydroxy-3-methyl-4-phenylbut-1-en-2-yl)boronic acid $[(S, R)-8 \mathrm{~d}]$}

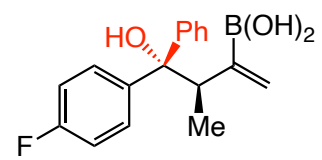

Synthesized according to the procedure described in section 8.2.1 with a freshly prepared solution of $\mathrm{PhMgBr}$ (0.34 M in THF), followed by $\mathrm{NaIO}_{4}$ workup ( $8 \mathrm{~h}$ ).

Colorless oil; $28.7 \mathrm{mg}, 0.0956$ mmol, 96\% yield, 98:2 d.r.; IR (neat): 3385 (br), 3056 (w), 2968 (w), 2929 (w), 1508 (s), 1492 (m), 1443 (s), 1280 (m), 1228 (m), 1160 (m), 979 (w), 934 (w), 834 (s), 699 (m), 575 (m) cm ${ }^{-1} ;{ }^{1} \mathbf{H}$ NMR (600 MHz, CDCl $): \delta 7.49-7.42$ (m, 2H), 7.28$7.17(\mathrm{~m}, 3 \mathrm{H}), 7.07$ (d, $J=7.5 \mathrm{~Hz}, 2 \mathrm{H}), 7.05-6.98(\mathrm{~m}, 2 \mathrm{H}), 5.76(\mathrm{~d}, J=2.3 \mathrm{~Hz}, 1 \mathrm{H}), 5.57$ (d, $J$ $=2.3 \mathrm{~Hz}, 1 \mathrm{H}), 3.64-3.54(\mathrm{~m}, 1 \mathrm{H}), 0.87(\mathrm{~d}, J=6.8 \mathrm{~Hz}, 3 \mathrm{H}) ;{ }^{13} \mathbf{C}$ NMR (151 MHz, CDCl $): \delta$ 
$162.1(\mathrm{~d}, J=246.2 \mathrm{~Hz}), 147.7$ (C-B bs), 143.4, 142.3 (d, $J=3.3 \mathrm{~Hz}), 128.8$ (d, $J=8.0 \mathrm{~Hz})$, 127.8, 127.1, 126.6, 122.3, 115.0 (d, $J=21.3 \mathrm{~Hz}), 89.6,49.4,17.7 ;{ }^{11}$ B NMR (160 MHz, $\left.\mathbf{C D C l}_{3}\right): \delta 32.1 ;{ }^{19} \mathbf{F}$ NMR (564 MHz, $\left.\mathbf{C D C l}_{3}\right): \delta-116.18--116.33$ (m); HRMS (DART): Calcd for $\mathrm{C}_{17} \mathrm{H}_{19} \mathrm{BO}_{2} \mathrm{~F}\left[\mathrm{M}+\mathrm{H}-\mathrm{H}_{2} \mathrm{O}\right]^{+}: 283.1300$, Found: 283.1297 ; specific rotation: $[\alpha]_{\mathrm{D}}{ }^{20}=$ $-118.6\left(c 1.0, \mathrm{CHCl}_{3}\right)$ for an enantiomerically enriched sample of 99:1 e.r., which corresponds to the e.r. of the ketone substrate $(\mathbf{2 m})$.

To determine whether there was any concomitant epimerization, the enantiomeric purity of alcohol $(\boldsymbol{S}, \boldsymbol{R})$-8d was determined by HPLC analysis of the derived $\beta$-hydroxy ketone (S3), in comparison with authentic racemic material (significant peak broadening with the boronic acid). Chiralcel OD-H column, 98:2 hexanes $/ i$-PrOH, $0.3 \mathrm{~mL} / \mathrm{min}, 220 \mathrm{~nm}$.
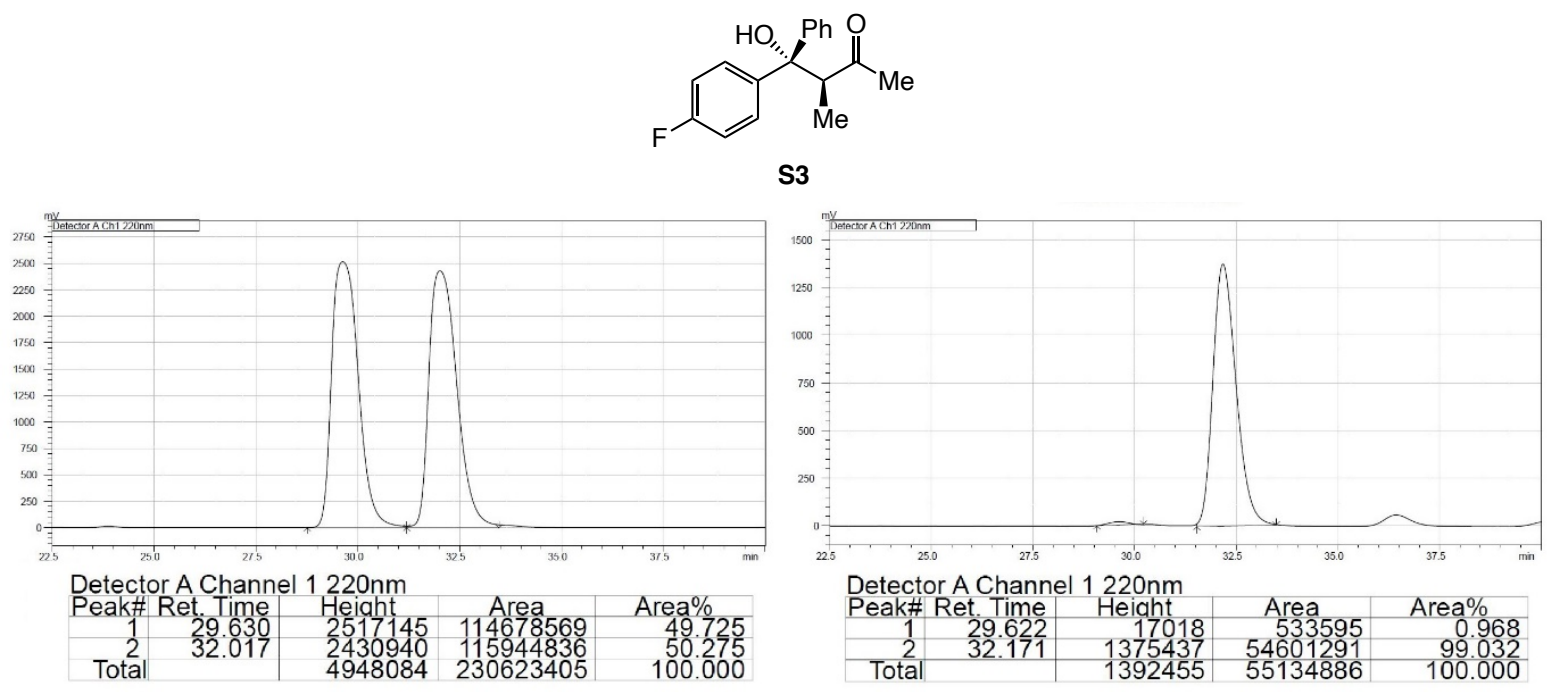

\begin{tabular}{|c|c|c|c|c|c|}
\hline Retention Time & Area & Area $\%$ & Retention Time & Area & Area\% \\
\hline 29.630 & 114678569 & 49.725 & 29.622 & 533595 & 0.968 \\
\hline 32.017 & 115944836 & 50.275 & 32.171 & 54601291 & 99.032 \\
\hline
\end{tabular}

8.5.11 ((3R,4R)-4-(4-Fluorophenyl)-4-hydroxy-3-methyl-4-phenylbut-1-en-2-yl)boronic acid $[(R, R)-8 d]$

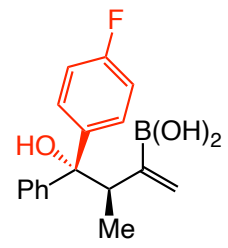

Synthesized according to the procedure described in section 8.2.1 with a freshly prepared solution of $4-\mathrm{F}-\mathrm{C}_{6} \mathrm{H}_{4} \mathrm{MgBr}\left(0.58 \mathrm{M}\right.$ in THF), followed by $\mathrm{NaIO}_{4}$ workup ( $8 \mathrm{~h}$ ).

Colorless oil; 28.4 mg, 0.0946 mmol, 95\% yield, >98:2 d.r.; IR (neat): 3384 (br), 3059 (w), 2969 (w), 2926 (w), 1506 (s), 1445 (s), 1398 (s), 1323 (m), 1292 (m), 1233 (m), 1161 (m), 979 (w), 936 (w), 831 (s), 698 (m) cm ${ }^{-1} ;{ }^{1} \mathbf{H}$ NMR (400 MHz, CDCl $): \delta 7.51-7.45$ (m, 2H), 7.387.32 (m, 2H), 7.32-7.27 (m, 1H), 7.09-7.02 (m, 2H), 6.96-6.89 (m, 2H), 5.75 (dd, $J=2.7,1.9$ $\mathrm{Hz}, 1 \mathrm{H}), 5.58$ (br s, 1H), $4.73(\mathrm{~s}, 1 \mathrm{H}), 3.63$ (qt, $J=6.9,2.7 \mathrm{~Hz}, 1 \mathrm{H}), 0.89$ (d, $J=6.9 \mathrm{~Hz}, 3 \mathrm{H})$; ${ }^{13}$ C NMR (151 MHz, CDCl $): \delta 161.8$ (d, $J=245.6$ Hz), 147.7 (C-B bs), 139.5 (d, $J=3.3$ 
$\mathrm{Hz}), 128.4,128.35,128.3,127.6,126.9,122.3,114.6$ (d, $J=21.3 \mathrm{~Hz}), 89.7,49.2,17.7 ;{ }^{11} \mathbf{B}$ NMR (160 MHz, $\left.\mathbf{C D C l}_{3}\right): \delta 32.0 ;{ }^{19} \mathbf{F}$ NMR (376 $\left.\mathbf{M H z}, \mathbf{C D C l}_{3}\right): \delta-117.20-117.42(\mathrm{~m})$; HRMS (DART): Calcd for $\mathrm{C}_{17} \mathrm{H}_{19} \mathrm{BO}_{2} \mathrm{~F}\left[\mathrm{M}+\mathrm{H}-\mathrm{H}_{2} \mathrm{O}\right]^{+}:$: 283.1300, Found: 283.1305; specific rotation: $[\alpha]_{\mathrm{D}}^{20}=-129.7\left(c 1.0, \mathrm{CHCl}_{3}\right)$ for an enantiomerically enriched sample of $99: 1$ e.r. which corresponds to the e.r. of the starting ketone (2m).

To determine whether there was any concomitant epimerization, the enantiomeric purity of alcohol $(\boldsymbol{R}, \boldsymbol{R})-\mathbf{8 d}$ was determined by HPLC analysis of the derived $\beta$-hydroxy ketone (S4), in comparison with authentic racemic material (significant peak broadening with the boronic acid). Chiralcel IC column, 99.5:0.5 hexanes $/ i-\mathrm{PrOH}, 1.0 \mathrm{~mL} / \mathrm{min}, 220 \mathrm{~nm}$.
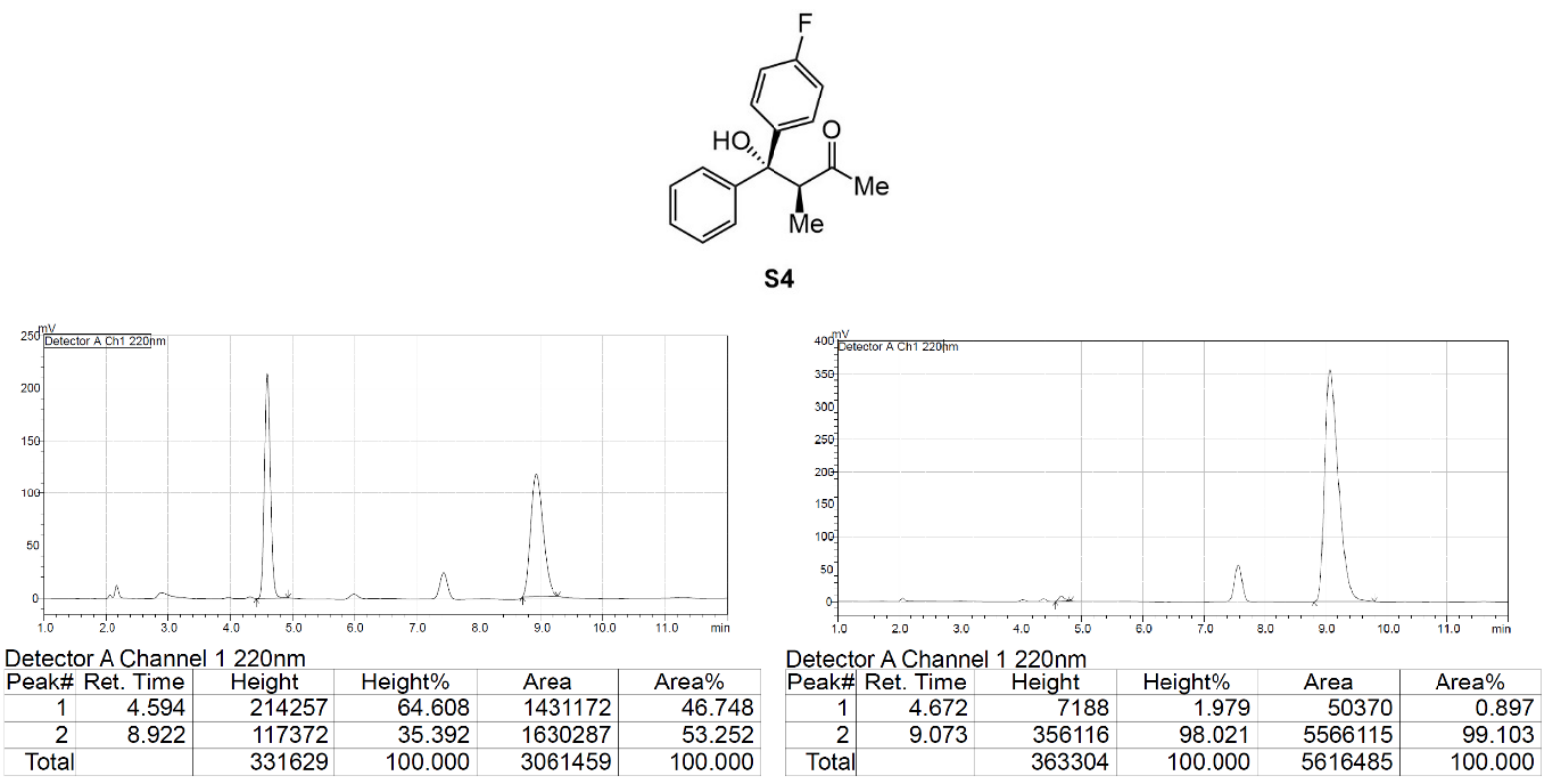

\begin{tabular}{|c|c|c|c|c|c|}
\hline Retention Time & Area & Area $\%$ & Retention Time & Area & Area $\%$ \\
\hline 4.594 & 1431172 & 46.748 & 4.672 & 50370 & 0.897 \\
\hline 8.922 & 1630287 & 53.252 & 9.073 & 5566115 & 99.103 \\
\hline
\end{tabular}

8.5.12 ((3R,4R)-4-Hydroxy-3-methyl-4-phenylhepta-1,6-dien-2-yl)boronic acid (9a)<smiles>C=CCC(O)(c1ccccc1)C(O)C(=O)O</smiles>

Synthesized according to the procedure described in section 8.2.4 with freshly prepared solution of allyl- $\mathrm{MgCl}(0.22 \mathrm{M}$ in THF, $0.15 \mathrm{mmol})$, followed by $\mathrm{NaIO}_{4}$ workup ( $\left.3 \mathrm{~h}\right)$.

Colorless oil; $21.8 \mathrm{mg}, 0.0886$ mmol, 89\% yield, >98:2 d.r.; IR (neat): 3369 (br), 3061 (w), 2969 (m), 2923 (w), 2852 (w), 1442 (s), 1401 (s), 1316 (s), 1295 (s), 1270 (s), 1006 (m), 927 (m), 867 (w), 760 (m), 699 (s) cm ${ }^{-1} ;{ }^{1} \mathbf{H}$ NMR (600 MHz, CDCl $): \delta 7.49-7.40$ (m, 2H), 7.39$7.30(\mathrm{~m}, 2 \mathrm{H}), 7.28-7.23(\mathrm{~m}, 1 \mathrm{H}), 5.78-5.42(\mathrm{~m}, 3 \mathrm{H}), 5.08-4.91(\mathrm{~m}, 2 \mathrm{H}), 2.81-2.67(\mathrm{~m}, 2 \mathrm{H})$, $2.40(\mathrm{dd}, J=14.6,6.8 \mathrm{~Hz}, 1 \mathrm{H}), 1.23(\mathrm{~d}, J=6.8 \mathrm{~Hz}, 3 \mathrm{H}) ;{ }^{13} \mathbf{C}$ NMR (151 MHz, CDCl $): \delta$ $145.6,133.5,128.4,127.0,124.9,121.1,118.4,87.8,52.6,41.2,12.7$. The carbon bearing the boron atom could not be detected due to quadrupolar effects; ${ }^{11} \mathbf{B}$ NMR (160 $\left.\mathbf{M H z}, \mathbf{C D C l}_{3}\right): \delta$ 
31.9; HRMS (DART): Calcd for $\mathrm{C}_{14} \mathrm{H}_{18} \mathrm{BO}_{2}\left[\mathrm{M}+\mathrm{H}-\mathrm{H}_{2} \mathrm{O}\right]^{+}$: 229.1394, Found: 229.1399; specific rotation: $[\alpha]_{\mathrm{D}}{ }^{20}=+80.9\left(c 1.0, \mathrm{CHCl}_{3}\right)$ for an enantiomerically enriched sample of 99:1 e.r., which corresponds to the e.r. of the starting ketone (2l).

8.5.13 ((3S,4R)-3-(((t-Butoxycarbonyl)amino)methyl)-4-hydroxy-4-methylhepta-1,6dien-2-yl)boronic acid (9b)

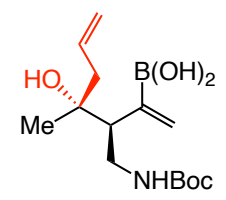

Synthesized according to the procedure described in section 8.2.4 with freshly prepared solution of allyl- $\mathrm{MgCl}(0.22 \mathrm{M}$ in THF, $0.15 \mathrm{mmol})$, followed by $\mathrm{NaIO}_{4}$ workup ( $\left.2 \mathrm{~h}\right)$.

Colorless oil; 20.9 mg, 0.0699 mmol, 70\% yield, 92:8 d.r.; IR (neat): 3347 (br), 2975 (m), 2928 (m), 1689 (s), 1510 (m), 1453 (m), 1407 (s), 1366 (m), 1278 (m), 1251 (m), 1168 (s), $1055(\mathrm{w}), 915(\mathrm{w}) \mathrm{cm}^{-1},{ }^{1} \mathbf{H}$ NMR (500 MHz, CD $\left.\mathbf{C N}\right): \delta 5.90-5.77(\mathrm{~m}, 1 \mathrm{H}), 5.73-5.62(\mathrm{~m}$, $1 \mathrm{H}), 5.60-5.41(\mathrm{~m}, 2 \mathrm{H}), 5.14-5.00(\mathrm{~m}, 2 \mathrm{H}), 3.37-3.07(\mathrm{~m}, 2 \mathrm{H}), 2.75-2.62(\mathrm{~m}, 1 \mathrm{H}), 2.56(\mathrm{~s}$, $1 \mathrm{H}), 2.34-2.15(\mathrm{~m}, 2 \mathrm{H}), 1.39(\mathrm{~s}, 9 \mathrm{H})$; broadened signals are due to equilibrium of carbamate rotamers; ${ }^{13} \mathbf{C}$ NMR (126 MHz, $\left.\mathbf{C D}_{3} \mathbf{C N}\right)$ : $\delta$ 156.8, 147.4-147.2 (m), 135.2, 122.1, 118.3, 83.4, $79.3,55.1,42.5,40.3,28.5,27.9$; The carbon bearing the boron atom could not be detected due to quadrupolar effects; ${ }^{11} \mathrm{~B}$ NMR (160 MHz, CD $\mathbf{C N}$ ): $\delta$ 31.9; HRMS (DART): Calcd for $\mathrm{C}_{14} \mathrm{H}_{27} \mathrm{BNO}_{3}\left[\mathrm{M}+\mathrm{H}-\mathrm{H}_{2} \mathrm{O}\right]^{+}: 282.1869$, Found: 282.1871 ; specific rotation: $[\alpha]_{\mathrm{D}}{ }^{20}=+3.7(c$ $1.0, \mathrm{CHCl}_{3}$ ) for an enantiomerically enriched sample of 97:3 e.r., which corresponds to the e.r. of the starting ketone $(4 \mathrm{~h})$.

8.5.14 (3R,4S)-4-hydroxy-3,4-dimethyl-3-phenylhepta-1,6-dien-2-yl)boronic acid (9c)

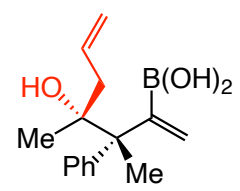

Synthesized according to the procedure described in section 8.2.4 with freshly prepared solution of allyl- $\mathrm{MgCl}(0.22 \mathrm{M}$ in THF, $0.15 \mathrm{mmol})$, followed by $\mathrm{NaIO}_{4}$ workup ( $\left.5 \mathrm{~h}\right)$.

Colorless oil; $31.3 \mathrm{mg}, 0.0819$ mmol, 82\% yield, 95:5 d.r.; IR (neat): 3354 (br), 3056 (w), 2974 (m), 2929 (w), 1494 (s), 1444 (s), 1399 (s), 1378 (m), 1320 (m), 1290 (m), 1158 (w), 1059 (w), 1047 (w), 917 (m), 758 (m), 703 (m) cm ${ }^{-1} ;{ }^{1}$ H NMR (500 MHz, CDCl $): \delta 7.42$ $7.27(\mathrm{~m}, 4 \mathrm{H}), 7.25-7.19(\mathrm{~m}, 1 \mathrm{H}), 5.96(\mathrm{~d}, J=1.8 \mathrm{~Hz}, 1 \mathrm{H}), 5.93-5.63(\mathrm{~m}, 1 \mathrm{H}), 5.57(\mathrm{~d}, J=1.8$ $\mathrm{Hz}, 1 \mathrm{H}), 5.13-5.02(\mathrm{~m}, 2 \mathrm{H}), 4.76(\mathrm{~s}, 1 \mathrm{H}), 2.51(\mathrm{dd}, J=14.0,5.8 \mathrm{~Hz}, 1 \mathrm{H}), 2.25(\mathrm{dd}, J=14.0$, $8.4 \mathrm{~Hz}, 1 \mathrm{H}), 1.50$ (s, 3H), 0.80 (s, 3H); ${ }^{13} \mathbf{C}$ NMR (126 MHz, CDCl $)$ : $\delta$ 144.4, 134.6, 128.7, $127.9,126.4,124.0,118.1,87.7,57.2,42.6,25.6,23.5$. The carbon bearing the boron atom could not be detected due to quadrupolar effects; ${ }^{11} \mathbf{B}$ NMR (160 MHz, $\left.\mathbf{C D C l}_{3}\right): \delta 31.2 ; \mathbf{H R M S}$ (DART): Calcd for $\mathrm{C}_{15} \mathrm{H}_{20} \mathrm{BO}_{2}\left[\mathrm{M}+\mathrm{H}-\mathrm{H}_{2} \mathrm{O}\right]^{+}:$: 243.1551, Found: 243.1548 ; specific rotation: $[\alpha]_{\mathrm{D}}{ }^{20}=-62.9\left(c 1.00, \mathrm{CHCl}_{3}\right)$ for an enantiomerically enriched sample of $>99: 1$ e.r. which corresponds to the enantiomeric purity of the starting ketone (5d). 


\subsubsection{5 (3S,4S)-4-Benzyl-4-hydroxy-3-((4-methoxybenzyl)oxy)hept-6-en-2-one (9d)}

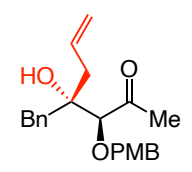

Synthesized according to the procedure described in section 8.2.4 with freshly prepared solution of allyl- $\mathrm{MgCl}(0.22 \mathrm{M}$ in THF, $0.15 \mathrm{mmol})$, followed by $\mathrm{NaBO}_{3} \cdot 4 \mathrm{H}_{2} \mathrm{O}$ workup (30 $\min )$.

Colorless oil; $53.2 \mathrm{mg}, 0.150$ mmol, 75\% yield, 97:3 d.r.; IR (neat): 3504 (bs), 2925 (w), 1705 (s), 1610 (w), 1512 (s), 1247 (s) cm ${ }^{-1} ;{ }^{1} \mathbf{H}$ NMR (600 MHz, CDCl $): \delta 7.31-7.21$ (m, 7H), 6.92 $(\mathrm{d}, J=8.2 \mathrm{~Hz}, 2 \mathrm{H}), 5.85-5.76(\mathrm{~m}, 1 \mathrm{H}), 5.20-5.01(\mathrm{~m}, 2 \mathrm{H}), 4.46(\mathrm{~d}, J=10.9 \mathrm{~Hz}, 1 \mathrm{H}), 4.31$ (d, $J=10.9 \mathrm{~Hz}, 1 \mathrm{H}), 3.83(\mathrm{~s}, 3 \mathrm{H}), 3.66(\mathrm{~s}, 1 \mathrm{H}), 2.82(\mathrm{~d}, J=14.0 \mathrm{~Hz}, 1 \mathrm{H}), 2.72(\mathrm{~d}, J=14.0 \mathrm{~Hz}$, $1 \mathrm{H}), 2.60$ (s, 1H), 2.42-2.37 (m, 1H), 2.27 (s, 3H), 2.25-2.19 (m, 1H); ${ }^{13}$ C NMR (151 MHz, $\left.\mathbf{C D C l}_{3}\right): \delta 213.3,159.7,136.6,133.4,131.1,129.8,129.2,128.2,126.7,119.2,114.1,87.2$, 76.2, 73.17, 55.4, 41.7, 41.0, 28.8; HRMS (DART): Calcd for $\mathrm{C}_{25} \mathrm{H}_{31} \mathrm{BO}_{5}[\mathrm{M}+\mathrm{H}]^{+}: 355.1893$, Found: 355.1904 ; specific rotation: $[\alpha]_{\mathrm{D}^{20}}=-13.2\left(c\right.$ 1.0, $\left.\mathrm{CHCl}_{3}\right)$ for an enantiomerically enriched sample of 98:2 e.r., which corresponds to the enantiomeric purity of the ketone starting material (6f).

To establish whether there was any concomitant epimerization, the enantiomeric purity of $9 \mathbf{d}$ was determined by HPLC analysis in comparison with authentic racemic material; $(R, R)$ Whelk-O1 column, 95.0:5.0 hexanes/i-PrOH, 0.5 mL/min, $220 \mathrm{~nm}$.
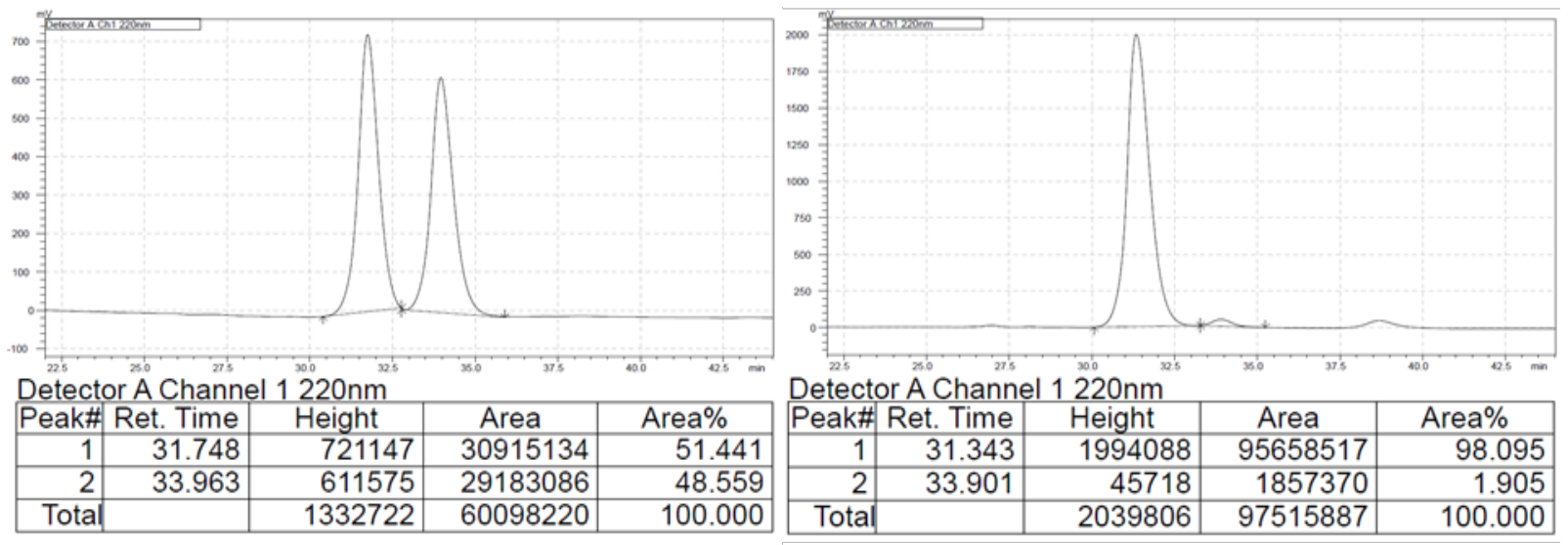

\begin{tabular}{|c|c|c|c|c|c|}
\hline Retention Time & Area & Area\% & Retention Time & Area & Area\% \\
\hline 31.748 & 30915134 & 51.441 & 31.343 & 95658517 & 98.095 \\
\hline 33.963 & 29183068 & 48.559 & 33.901 & 1857370 & 1.905 \\
\hline
\end{tabular}

8.5.16 $(R)-5$-Allyl-5-((R)-5-((t-butyldimethylsilyl)oxy)-2-(4,4,5,5-tetramethyl-1,3,2dioxaborolan-2-yl)pent-1-en-3-yl)dihydrofuran-2(3H)-one (9e; intermediate for $4 \mathrm{~d} \rightarrow \mathbf{1 3}$, Scheme 12)

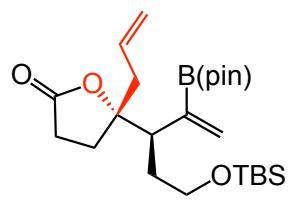


A modified version of the aforementioned general protocol was used. The reaction was performed at $-78{ }^{\circ} \mathrm{C}$ for $1 \mathrm{~h}$ and then the mixture was allowed to warm to $22{ }^{\circ} \mathrm{C}$ for $30 \mathrm{~min}$, after which it was again allowed to warm to $60^{\circ} \mathrm{C}$ for $30 \mathrm{~min}$ (to promote lactone formation). The mixture was cooled to $22^{\circ} \mathrm{C}$, and the reaction was quenched and the product was used for the next step without purification. It should however be noted that the absence of a free hydroxyl group renders the compound stable in silica gel chromatography.

Yellow oil; 33.4 mg, 0.0741 mmol, 74\% yield, 92:8 d.r.; IR (neat): 2952 (m), 2928 (m), 2897 (w), 2855 (m), 1774 (s), 1420 (m), 1371 (m), $1311(\mathrm{~m}), 1211$ (m), 1141 (s), 1096 (s), $945(\mathrm{w})$, 919 (w), 835 (s), 775 (m) cm ${ }^{-1},{ }^{1}$ H NMR (500 MHz, CDCl $)$ ): $\delta 6.04-5.98$ (m, 1H), 5.82-5.66 $(\mathrm{m}, 2 \mathrm{H}), 5.18-5.08(\mathrm{~m}, 2 \mathrm{H}), 3.62-3.56(\mathrm{~m}, 1 \mathrm{H}), 3.45-3.36(\mathrm{~m}, 1 \mathrm{H}), 2.77-2.68(\mathrm{~m}, 1 \mathrm{H}), 2.58-$ $2.43(\mathrm{~m}, 3 \mathrm{H}), 2.33-2.19(\mathrm{~m}, 2 \mathrm{H}), 2.01-1.85(\mathrm{~m}, 2 \mathrm{H}), 1.82-1.71(\mathrm{~m}, 1 \mathrm{H}), 1.25(\mathrm{~s}, 6 \mathrm{H}), 1.24(\mathrm{~s}$, 6H), 0.87 (s, 10H), 0.01 (s, 3H), 0.00 (s, 3H); ${ }^{13} \mathbf{C}$ NMR (151 MHz, CDCl 3 ): $\delta 177.1,133.3$, 132.2, 120.1, 89.9, 83.8, 61.2, 48.8, 42.1, 30.8, 29.6, 27.9, 26.1, 25.0, 24.7, 18.4, -5.1, -5.2; the carbon bearing the boron atom could not be detected due to quadrupolar effects; ${ }^{11} \mathbf{B}$ NMR (160 MHz, CDCl $\mathbf{~}_{3}$ ): $\delta 30.9$; HRMS (DART): Calcd for $\mathrm{C}_{24} \mathrm{H}_{44} \mathrm{BO}_{5} \mathrm{Si}[\mathrm{M}+\mathrm{H}]^{+}:$451.3046, Found: 451.3048; specific rotation: $[\alpha]_{\mathrm{D}}{ }^{20}=-8.0\left(c 1.0, \mathrm{CHCl}_{3}\right)$ for an enantiomerically enriched sample of 97:3 e.r., which corresponds to the enantiomeric purity of ketone (4d).

Enantiomeric purity was determined by HPLC analysis in comparison with authentic racemic material; Chiralcel OZ-H column, 99.5:0.5 hexanes $/ i$-PrOH, $0.6 \mathrm{~mL} / \mathrm{min}, 220 \mathrm{~nm}$.
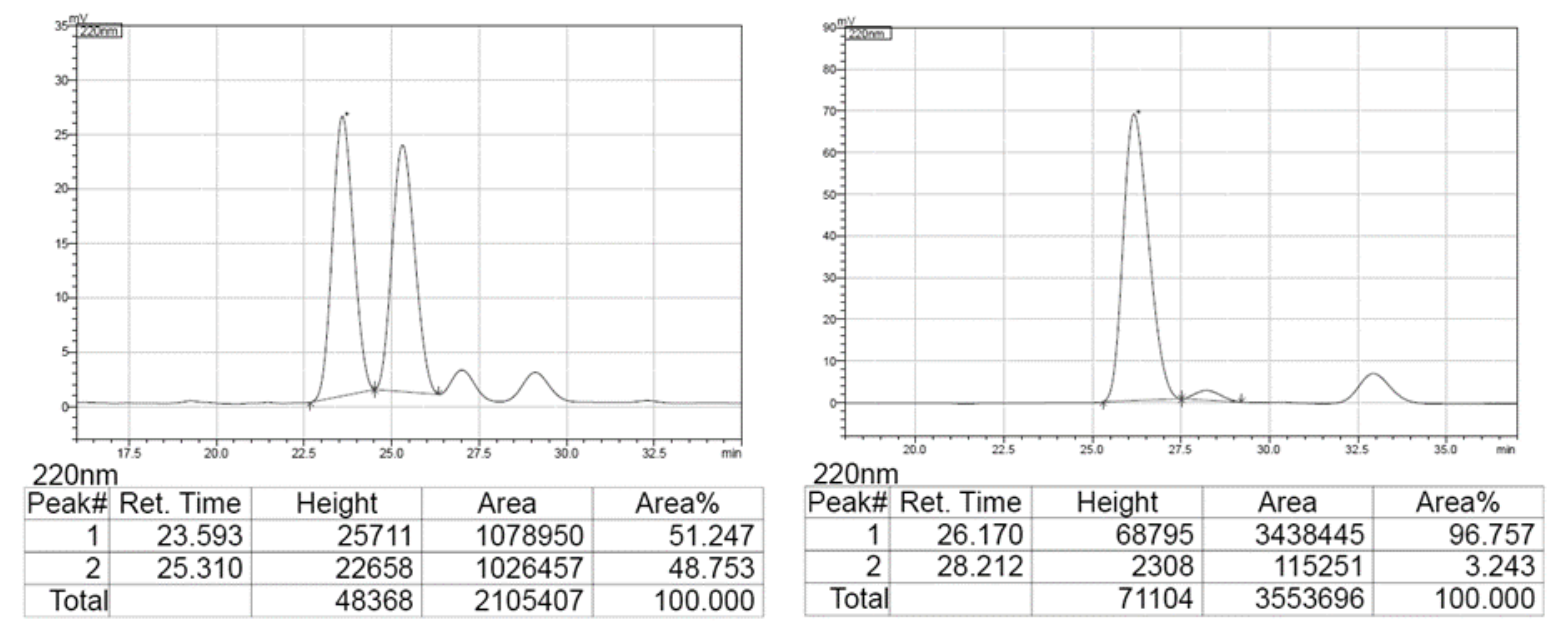

\begin{tabular}{|c|c|c|c|c|c|}
\hline Retention Time & Area & Area\% & Retention Time & Area & Area $\%$ \\
\hline 23.593 & 1078950 & 51.247 & 26.170 & 3438445 & 96.757 \\
\hline 25.310 & 2105407 & 48.753 & 28.212 & 115251 & 3.243 \\
\hline
\end{tabular}

8.5.17 ((3R,4R)-3-(2-((t-Butyldimethylsilyl)oxy)ethyl)-4-hydroxy-4-phenylhexa-1,5-dien2-yl)boronic acid (10)

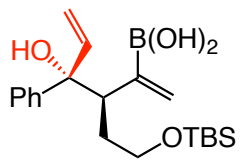


Synthesized according to the procedure described in section 8.2.1 with commercially available vinyl- $\mathrm{MgBr}$ (1.0 M in THF), followed by $\mathrm{NaIO}_{4}$ workup ( $2 \mathrm{~h}$ ). was used; $\mathrm{NaIO}_{4}$ workup, $2 \mathrm{~h}$. Colorless oil; 34.9 mg, 0.0880 mmol, 88\% yield, 97:3 d.r.; IR (neat): 3378 (w), 2950 (m), 2926 (m), 2854 (m), 1445 (m), 1403 (m), 1255 (m), 1097 (m), 929 (m), 834 (s), 775 (m), 699

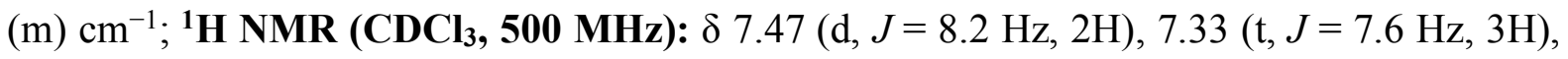
7.26-7.23 (m, 1H), 6.11 (dd, $J=16.9,10.7 \mathrm{~Hz}, 1 \mathrm{H}), 5.71(\mathrm{~s}, 1 \mathrm{H}), 5.57(\mathrm{~s}, 1 \mathrm{H}), 5.26-5.13$ (m, $2 \mathrm{H}), 4.72(\mathrm{~s}, 1 \mathrm{H}), 3.77-3.62(\mathrm{~m}, 2 \mathrm{H}), 3.29-3.22(\mathrm{~m}, 1 \mathrm{H}), 2.02-1.92(\mathrm{~m}, 1 \mathrm{H}), 1.66-1.55(\mathrm{~m}$, 1H), 0.88 (s, 9H), 0.03 (s, 6H); ${ }^{13} \mathbf{C}$ NMR (CDCl 3 , 101 MHz): $\delta$ 145.9(C-B, bs), 144.8, 139.7, $128.3,127.4,125.9,122.6,114.3,87.8,61.1,52.2,33.9,26.1,18.4,-5.17,-5.21 ;{ }^{11} \mathbf{B}$ NMR (CDCl 3 , 160 MHz): $\delta 31.1$; HRMS (DART): Calcd for $\mathrm{C}_{20} \mathrm{H}_{32} \mathrm{BO}_{3} \mathrm{Si}\left[\mathrm{M}+\mathrm{H}-\mathrm{H}_{2} \mathrm{O}\right]^{+}: 359.2208$, Found: 359.2204; specific rotation: $[\alpha]_{\mathrm{D}^{20}}=-34.6\left(c\right.$ 1.0, $\left.\mathrm{CHCl}_{3}\right)$ an enantiomerically enriched sample of 98:2 e.r., which corresponds to the enantiomeric purity of ketone (2k).

\subsubsection{8 ((3R,4S)-3-(2-((t-Butyldimethylsilyl)oxy)ethyl)-4-hydroxy-4-} (trimethylsilyl)ethynyl)hepta-1,6-dien-2-yl)boronic acid (11)

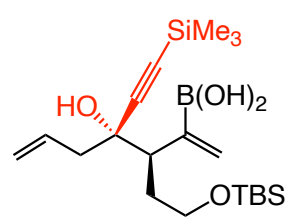

Synthesized according to the procedure described in section 8.2.1 with a freshly prepared solution of the alkynyl- $\mathrm{Li}$ compound ( 1.8 equiv. in $0.5 \mathrm{~mL}$ of THF, prepared at $-78{ }^{\circ} \mathrm{C}$ ), followed by $\mathrm{NaIO}_{4}$ workup ( $2 \mathrm{~h}$ ).

Colorless oil; $34.9 \mathrm{mg}, 0.0850 \mathrm{mmol}, 85 \%$ yield, 97:3 d.r.; IR (neat): 3351 (br), 2925 (m), 2152 (w), 1644 (w), 1401 (m), 1245 (m), 1098 (m), 839 (s), 774 (m) cm ${ }^{-1} ;{ }^{1}$ H NMR (400 MHz, CDCl $): \delta 5.99-5.84(\mathrm{~m}, 1 \mathrm{H}), 5.78(\mathrm{~s}, 1 \mathrm{H}), 5.63(\mathrm{~s}, 1 \mathrm{H}), 5.18-5.08(\mathrm{~m}, 2 \mathrm{H}), 3.82-3.63$ (m, 2H), 2.84-2.72 (m, 1H), 2.59-2.41 (m, 2H), 2.06-1.93 (m, 1H), 1.79-1.65 (m, 1H), 0.90 $(\mathrm{s}, 9 \mathrm{H}), 0.16(\mathrm{~s}, 9 \mathrm{H}), 0.06(\mathrm{~s}, 6 \mathrm{H}) ;{ }^{13} \mathbf{C}$ NMR (151 MHz, $\left.\mathbf{C D C l}_{3}\right): \delta 145.5,132.9,123.7,119.0$, 104.6, 92.7, 82.5, 60.7, 49.2, 47.3, 34.9, 26.1, 18.4, 0.0, -5.1; ${ }^{11} \mathbf{B}$ NMR (128 MHz, CDCl $\left.\mathbf{3}\right)$ : $\delta$ 29.7; HRMS (ESI): Calcd for $\mathrm{C}_{20} \mathrm{H}_{38} \mathrm{BO}_{3} \mathrm{Si}_{2}\left[\mathrm{M}+\mathrm{H}-\mathrm{H}_{2} \mathrm{O}\right]^{+}:$: 393.2447, Found: 393.2444; specific rotation: $[\alpha]_{\mathrm{D}^{20}}=+22.7\left(c 1.0, \mathrm{CHCl}_{3}\right)$ for an enantiomerically enriched sample of 97:3 e.r., which corresponds to the enantiomeric purity of the ketone (4m).

To establish whether there was any concomitant epimerization, the enantiomeric purity of the $\beta$-hydroxy ketone $\mathbf{S} 4$ was determined by HPLC analysis in comparison with authentic racemic material; $(R, R)$-Whelk-O1 column, 99.0:0.5 hexanes $/ i-\mathrm{PrOH}, 0.5 \mathrm{~mL} / \mathrm{min}, 220 \mathrm{~nm}$.

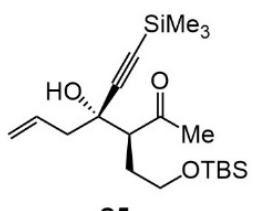



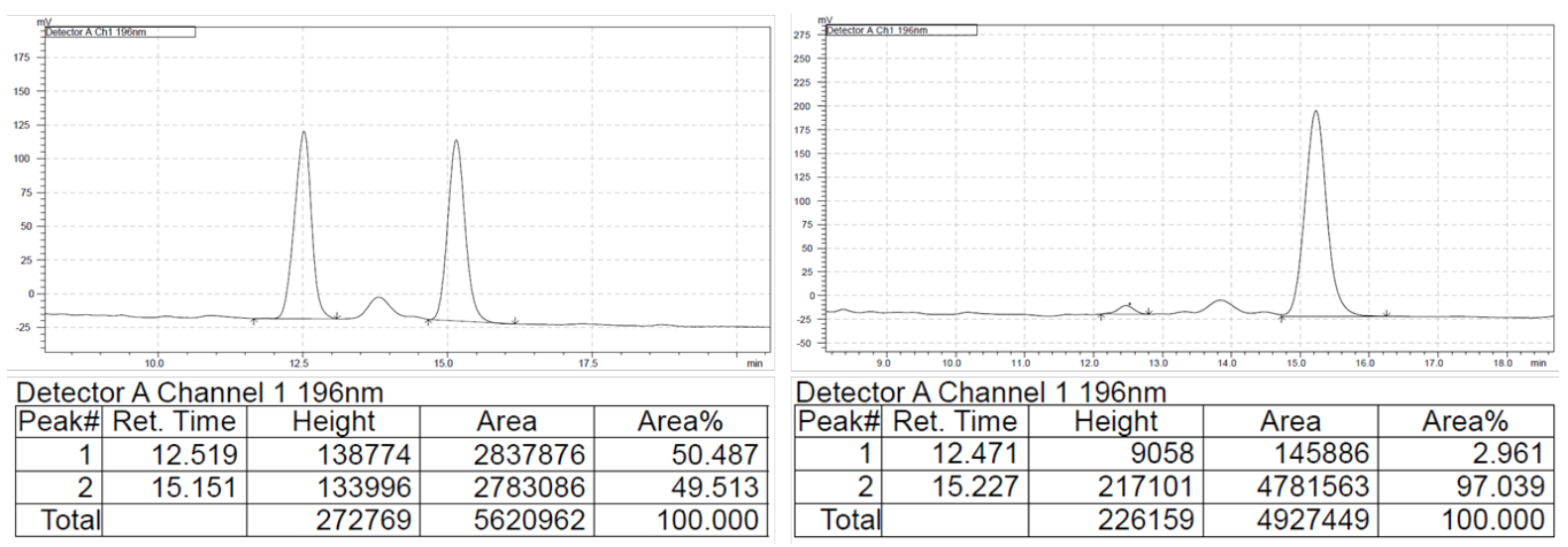

\begin{tabular}{|c|c|c|c|c|c|}
\hline Retention Time & Area & Area\% & Retention Time & Area & Area $\%$ \\
\hline 12.519 & 2837876 & 50.487 & 12.471 & 145886 & 2.961 \\
\hline 15.151 & 2783086 & 49.513 & 15.227 & 4781563 & 97.039 \\
\hline
\end{tabular}

8.5.19 (3S,4R)-3-(2-((t-butyldimethylsilyl)oxy)ethyl)-4-hydroxy-4-methyl-7(trimethylsilyl)hept-6-yn-2-one (12a)

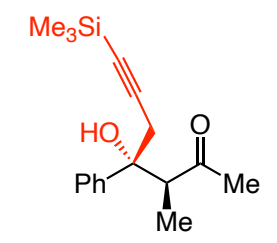

Prepared according to the procedure described in section 8.2.6 with a freshly prepared solution of $\mathrm{Me}_{3} \mathrm{Si}$-substituted propargyl- $\mathrm{ZnBr}$ (2.0 equiv, $1.0 \mathrm{M}$ solution in THF) followed by followed by $\mathrm{NaBO}_{3} \bullet 4 \mathrm{H}_{2} \mathrm{O}$ workup (30 min).

Colorless oil; $21.0 \mathrm{mg}, 0.0730 \mathrm{mmol}, 73 \%$ yield, >98:2 d.r.; IR (neat): 3466 (br), 2958 (w), 2177 (w), 1699 (m), 1248 (m), 838 (s), 759 (s), 699 (s) $\mathrm{cm}^{-1} ;{ }^{1}$ H NMR (600 MHz, CDCl $)$ ): $\delta$ $7.58-7.51(\mathrm{~m}, 2 \mathrm{H}), 7.35-7.28(\mathrm{~m}, 2 \mathrm{H}), 7.28-7.21(\mathrm{~m}, 1 \mathrm{H}), 4.33(\mathrm{~s}, 1 \mathrm{H}), 3.61(\mathrm{q}, \mathrm{J}=7.1 \mathrm{~Hz}$, 1H), $2.74(\mathrm{~d}, \mathrm{~J}=16.9 \mathrm{~Hz}, 1 \mathrm{H}), 2.55(\mathrm{~d}, \mathrm{~J}=16.9 \mathrm{~Hz}, 1 \mathrm{H}), 2.04(\mathrm{~s}, 3 \mathrm{H}), 1.31(\mathrm{~d}, \mathrm{~J}=7.1 \mathrm{~Hz}, 3 \mathrm{H})$, 0.12 (s, 9H); ${ }^{13} \mathbf{C}$ NMR (151 MHz, $\left.\mathbf{C D C l}_{3}\right): \delta 215.5,145.7,128.1,127.3,125.3,102.7,88.4$, 76.0, 50.9, 32.2, 31.4, 11.7, 0.0; HRMS (ESI): Calcd for $\mathrm{C}_{17} \mathrm{H}_{24} \mathrm{O}_{2} \mathrm{Si}[\mathrm{M}+\mathrm{Na}]^{+}: 311.144328$, Found: 311.144409 ; specific rotation: $[\alpha]_{\mathrm{D}}{ }^{20}=+89.8\left(c 1.0, \mathrm{CHCl}_{3}\right)$ for an enantiomerically enriched sample of 99:1 e.r., which corresponds to the enantiomeric purity of ketone (2l).

8.5.20 (R)-5-((S)-1-((t-butyldimethylsilyl)oxy)-4-oxopentan-3-yl)-5-(3(trimethylsilyl)prop-2-yn-1-yl)dihydrofuran-2(3H)-one (12b)

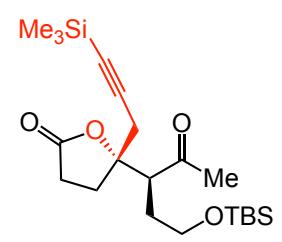

Synthesized according to the procedure described in section 8.2.6 with a freshly prepared solution of $\mathrm{Me}_{3} \mathrm{Si}$-substituted propargyl- $\mathrm{ZnBr}$ (2.0 equiv., 1.0 M solution in THF) followed by followed by $\mathrm{NaBO}_{3} \bullet 4 \mathrm{H}_{2} \mathrm{O}$ workup $(30 \mathrm{~min})$. 
Colorless oil; 4.20 g, 10.2 mmol, 78\% yield, >98:2 d.r.; IR (neat): 2955 (w), 2930 (w), 2857 (w), 2178 (w), 1780 (s), 1713 (m), 1359 (w), 1250 (s), 1183 (m), 1092 (s), 1029(m), 937 (w), 832 (s), 775 (s) cm ${ }^{-1}$; ${ }^{1} \mathbf{H}$ NMR (CDCl, 400 MHz): $\delta 3.60$ (1H, ddd, $J=10.6,6.1,5.0 \mathrm{~Hz}$ ), $3.50(1 \mathrm{H}, \mathrm{ddd}, J=10.6,7.5,4.4 \mathrm{~Hz}, 1 \mathrm{H}), 3.19(1 \mathrm{H}, \mathrm{dd}, J=10.5,2.5 \mathrm{~Hz}), 2.79(1 \mathrm{H}$, ddd, $J=$ $18.2,10.8,8.5 \mathrm{~Hz}), 2.71(1 \mathrm{H}, \mathrm{d}, J=17.3 \mathrm{~Hz}), 2.55(1 \mathrm{H}, \mathrm{ddd}, J=18.2,10.9,4.8 \mathrm{~Hz}), 2.48(1 \mathrm{H}$, d, $J=17.3 \mathrm{~Hz}), 2.38-2.23(2 \mathrm{H}, \mathrm{m}), 2.28(3 \mathrm{H}, \mathrm{s}), 1.92(1 \mathrm{H}, \mathrm{dddd}, J=13.5,10.5,6.1,4.4 \mathrm{~Hz})$, $1.55(1 \mathrm{H}$, dddd, $J=13.5,7.5,5.0,2.5 \mathrm{~Hz}), 0.86(9 \mathrm{H}, \mathrm{s}), 0.12(9 \mathrm{H}, \mathrm{s}), 0.02(3 \mathrm{H}, \mathrm{s}), 0.01(3 \mathrm{H}$, s); $\left.{ }^{13} \mathbf{C ~ N M R ~ ( C D C l} 3 \mathbf{3}, \mathbf{1 0 1} \mathbf{M H z}\right): \delta 209.5,176.1,100.8,89.1,87.0,61.2,56.5,33.8,30.9,30.1$, 29.4, 29.1, 26.0, 18.4, -0.1, -5.36, -5.40; HRMS (ESI): Calcd for $\mathrm{C}_{21} \mathrm{H}_{39} \mathrm{O}_{4} \mathrm{Si}_{2}[\mathrm{M}+\mathrm{H}]^{+}$: 411.2381, Found: 411.2372; specific rotation: $\left.[\alpha]_{\mathrm{D}}{ }^{20}=+50.7(c) 1.0, \mathrm{CHCl}_{3}\right)$ for an enantiomerically enriched sample of 98:2 e.r. which corresponds to the enantiomeric purity of the ketone (4d).

\section{Product Functionalization}

\subsection{Catalytic ring-closing metathesis}

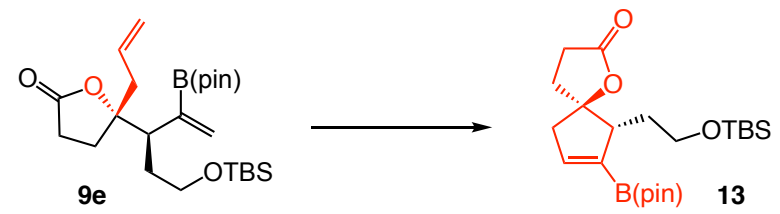

A Schlenk tube was charged with 1,3-Bis-(2,4,6-trimethylphenyl)-2imidazolidinylidene)dichloro(o-isopropoxyphenylmethylene)ruthenium $(1.4 \mathrm{mg}, \quad 0.0021$ $\mathrm{mmol})$ and $9 \mathrm{e}(19.0 \mathrm{mg}, 0.042 \mathrm{mmol}, 92: 8 \mathrm{~d}$.r.). The tube was sealed with a septum and toluene $(5 \mathrm{~mL})$ was added, after which the mixture was allowed to heat to $90{ }^{\circ} \mathrm{C}$ for $3.5 \mathrm{~h}(>98 \%$ conv., as judged by TLC analysis). The volatiles were removed in vacuo to afford brown oil, which was purified by silica gel chromatography $\left(\mathrm{R}_{f}=0.25,5: 1\right.$ hexanes/EtOAc) to afford $\mathbf{1 3}$ as colorless oil (16.9 $\mathrm{mg}, 0.040 \mathrm{mmol}, 95 \%$ yield). Diastereomeric purity (92:8 d.r.) was determined by analysis of the ${ }^{1} \mathrm{H}$ NMR spectrum $\left(\mathrm{CDCl}_{3}\right)$.

\subsection{1 (5R,6R)-6-(2-((t-Butyldimethylsilyl)oxy)ethyl)-7-(4,4,5,5-tetramethyl-1,3,2-} dioxaborolan-2-yl)-1-oxaspiro[4.4]non-7-en-2-one (13)

Colorless oil; 16.9 mg, 0.040 mmol, 95\% yield, 92:8 d.r.; IR (neat): 2924 (m), 2852 (m), 1774 (s), 1614 (w), 1371 (s), 1143 (m), 834 (m) cm ${ }^{-1}$; ${ }^{1} \mathbf{H}$ NMR (600 MHz, CDCl $\left.{ }_{3}\right): \delta 6.50(\mathrm{~s}, 1 \mathrm{H})$, 3.70-3.62 (m, 1H), 3.61-3.52 (m, 1H), 2.94-2.81 (m, 1H), $2.65(\mathrm{~s}, 2 \mathrm{H}), 2.60-2.53(\mathrm{~m}, 2 \mathrm{H})$, 2.42 (dt, J = 13.1, $8.4 \mathrm{~Hz}, 1 \mathrm{H}), 2.19-2.10(\mathrm{~m}, 1 \mathrm{H}), 1.85-1.76(\mathrm{~m}, 1 \mathrm{H}), 1.60-1.45$ (m, 1H), 1.25 (s, 12H), 0.88 (s, 9H), 0.04 (s, 6H); ${ }^{13} \mathbf{C}$ NMR (151 MHz, CDCl 3 ): $\delta 176.6,143.8,138.7,97.4$, 83.5, 61.8, 52.1, 45.7, 34.0, 29.9, 28.6, 26.1, 25.1, 24.8, 18.6, -5.1; ${ }^{11}$ B NMR (160 MHz, $\mathbf{C D C l}_{3}$ ): $\delta 28.5$; HRMS (ESI): Calcd for $\mathrm{C}_{22} \mathrm{H}_{40} \mathrm{BO}_{5} \mathrm{Si}[\mathrm{M}+\mathrm{H}]^{+}:$423.2724, Found: 423.2735; specific rotation: $[\alpha]_{\mathrm{D}}{ }^{20}=+44.4\left(\mathrm{c} 1.0, \mathrm{CHCl}_{3}\right)$ for an enantiomerically enriched sample of 98:2 e.r., which corresponds to the enantiomeric purity of alcohol (9e). 


\subsection{Boryl to carboxyl conversion/cyclization}

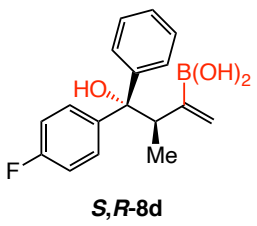

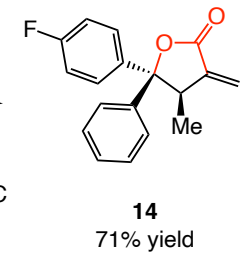

A flame-dried round-bottom flask containing a stir bar was charged with $\mathrm{Pd}(\mathrm{OAc})_{2}(4.50 \mathrm{mg}$, $0.02 \mathrm{mmol})$ and Xantphos (14.0 mg, $24.0 \mu \mathrm{mol})$, DMSO (1 mL), and $\mathrm{MeOH}(1 \mathrm{~mL})$. The mixture was allowed to stir at $22^{\circ} \mathrm{C}$ for $10 \mathrm{~min}$, after which vinylic boronic acid $(\boldsymbol{S}, \boldsymbol{R})-\mathbf{8 d}(120$ $\mathrm{mg}, 0.40 \mathrm{mmol}$ ) and 1,4-benzoquinone $(47.5 \mathrm{mg}, 0.44 \mathrm{mmol})$ were added as a mixture in a solution of 1:1 DMSO:MeOH (2 mL). The solution was purged three times with an atmosphere of $\mathrm{CO}$, and the mixture was allowed to stir at $50{ }^{\circ} \mathrm{C}$ for $14 \mathrm{~h}$ under an atmosphere of $\mathrm{CO}$ (balloon). The solution was then allowed to cool to $22{ }^{\circ} \mathrm{C}$ and filtered through Celite with $\mathrm{Et}_{2} \mathrm{O}$ $(3 \times 5 \mathrm{~mL})$ as the eluent. The filtrate was diluted with $\mathrm{H}_{2} \mathrm{O}(10 \mathrm{~mL})$, the two phases were separated and the aqueous phase was washed with $\mathrm{Et}_{2} \mathrm{O}(3 \times 5 \mathrm{~mL})$. The combined organic layers were dried over $\mathrm{MgSO}_{4}$, filtered and the volatiles were removed in vacuo to afford brown oil residue, which was purified by silica gel chromatography $\left(9: 1 \rightarrow 5: 1\right.$ hexanes:Et $\left._{2} \mathrm{O}\right)$ to furnish lactone 14 as colorless oil ( $80 \mathrm{mg}, 283 \mu \mathrm{mol}, 71 \%$ yield).

\subsection{1 (4R,5S)-5-(4-Fluorop.enyl)-4-methyl-3-methylene-5-phenyldihydrofuran-2(3H)- one (14)}

Colorless oil; $20.1 \mathrm{mg}, 0.0710$ mmol, 71\% yield, 98:2 d.r.; IR (neat): 3060 (w), 2975 (w), 2929 (w), 1766 (s), 1509 (m), 1447 (w), 1263 (m), 1233 (m), 1158 (m), 983 (m), 967 (m), 844 (w), $814(\mathrm{w}), 700(\mathrm{~m}) \mathrm{cm}^{-1}{ }^{1}{ }^{1} \mathbf{H}$ NMR (400 MHz, $\left.\mathbf{C D C l}_{3}\right): \delta 7.51-7.44(\mathrm{~m}, 2 \mathrm{H}), 7.32-7.23(\mathrm{~m}, 4 \mathrm{H})$, $7.12-7.00(\mathrm{~m}, 4 \mathrm{H}), 6.30(\mathrm{~d}, J=2.9 \mathrm{~Hz}, 1 \mathrm{H}), 5.56(\mathrm{~d}, J=2.6 \mathrm{~Hz}, 1 \mathrm{H}), 3.88-3.78(\mathrm{~m}, 1 \mathrm{H}), 1.02$ $(\mathrm{d}, J=6.9 \mathrm{~Hz}, 3 \mathrm{H}) ;{ }^{13} \mathbf{C}$ NMR (151 MHz, $\left.\mathbf{C D C l}_{3}\right): \delta 169.7,162.6(\mathrm{~d}, J=248.1 \mathrm{~Hz}), 140.6(\mathrm{~d}$, $J=33.7 \mathrm{~Hz}), 138.8(\mathrm{~d}, J=3.5 \mathrm{~Hz}), 128.82,128.76,128.3,128.0,126.5,121.8,115.6(\mathrm{~d}, J=$ $21.4 \mathrm{~Hz}), 90.1,44.0,16.9 ;{ }^{19} \mathbf{F}$ NMR (376 $\left.\mathbf{M H z}, \mathbf{C D C l}_{3}\right): \delta-113.32-113.44(\mathrm{~m})$; HRMS (DART): Calcd for $\mathrm{C}_{18} \mathrm{H}_{16} \mathrm{O}_{2} \mathrm{~F}[\mathrm{M}+\mathrm{H}]^{+}: 283.1129$, Found: 283.1134 ; specific rotation: $[\alpha]_{\mathrm{D}}{ }^{20}$ $=-130.6\left(c\right.$ 1.0, $\left.\mathrm{CHCl}_{3}\right)$ for an enantiomerically enriched sample of 99:1 e.r., which corresponds to the enantiomeric purity of the starting material alcohol $(\boldsymbol{S}, \boldsymbol{R})-\mathbf{8 d}$.

\section{$9.3 \mathrm{C}-\mathrm{B}$ to $\mathrm{C}-\mathrm{H}$ conversion}

\subsection{1 (3S,4R)-4-(2-((t-Butyldimethylsilyl)oxy)ethyl)-2,2,3-trimethylhex-5-en-3-ol (15a)}

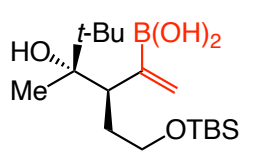

7c

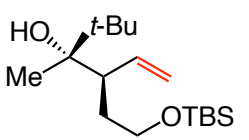

$15 a$

A previously reported procedure was used ${ }^{34}$. A flame-dried round-bottom flask with a stir bar was charged with 1,3-dicyclohexylimidazolium tetrafluoroborate $(1.60 \mathrm{mg}, 5.00 \mu \mathrm{mol}), \mathrm{CuCl}$ $(0.50 \mathrm{mg}, 5.00 \mu \mathrm{mol}), \mathrm{NaOMe}(6.50 \mathrm{mg}, 0.12 \mathrm{mmol})$, and THF $(0.5 \mathrm{~mL})$. The mixture was allowed to stir at $22{ }^{\circ} \mathrm{C}$ for $1 \mathrm{~h}$, after which a solution of alkenyl boronic acid 7c $(34 \mathrm{mg}, 0.10$ 
$\mathrm{mmol})$ and $\mathrm{MeOH}(20 \mu \mathrm{L}, 0.50 \mathrm{mmol})$, dissolved in THF $(0.5 \mathrm{~mL})$ was added, and the mixture was allowed to stir for $4 \mathrm{~h}$ at $60{ }^{\circ} \mathrm{C}$. The mixture was then allowed to cool to $22{ }^{\circ} \mathrm{C}$ and filtered through a short plug of Celite and silica gel $\left(3 \times 2 \mathrm{~mL} \mathrm{Et}_{2} \mathrm{O}\right)$. The filtrate was concentrated under reduced pressure, affording colorless oil, which was purified by silica gel chromatography $\left(20: 1 \rightarrow 10: 1\right.$ hexanes: Et $\left._{2} \mathrm{O}\right)$ to afford 15a.

Colorless oil; $28.0 \mathrm{mg}, 0.0931 \mathrm{mmol}, 93 \%$ yield, 98:2 d.r.; IR (neat): 3479 (br), 2955 (s), 2928 (m), 2858 (m), 1472 (w), 1374 (w), 1256 (m), 1091 (s), 1005 (w), 911 (m), 835 (s), 775 (m), $\mathrm{cm}^{-1}$; ${ }^{1} \mathbf{H}$ NMR (400 MHz, $\left.\mathbf{C D C l}_{3}\right): \delta 5.70(1 \mathrm{H}, \mathrm{dt}, J=16.9,10.2 \mathrm{~Hz}), 5.01-4.90(2 \mathrm{H}, \mathrm{m})$, $3.67(1 \mathrm{H}, \mathrm{ddd}, J=10.0,6.5,3.7 \mathrm{~Hz}), 3.51(1 \mathrm{H}, \mathrm{td}, J=10.0,5.3 \mathrm{~Hz}, 1 \mathrm{H}), 2.52(1 \mathrm{H}, \mathrm{ddd}, J=$ 10.0, 8.5, 3.0 Hz), 2.17-2.05 (1H, m), $1.87(1 \mathrm{H}, \mathrm{bs}), 1.33-1.20(1 \mathrm{H}, \mathrm{m}), 1.11(3 \mathrm{H}, \mathrm{s}), 0.97(9 \mathrm{H}$, s), 0.90 (9H, s), 0.05 (6H, s); ${ }^{13} \mathbf{C}$ NMR (101 MHz, $\left.\mathbf{C D C l}_{3}\right): \delta 141.7,115.0,77.0,61.5,48.7$, 39.6, 33.4, 26.4, 26.1 18.4, 18.3, -5.19, -5.20; HRMS (ESI): Calcd for $\mathrm{C}_{17} \mathrm{H}_{37} \mathrm{O}_{2} \mathrm{Si}[\mathrm{M}+\mathrm{H}]^{+}$: 301.2557, Found: 301.2563 ; specific rotation: $[\alpha]_{\mathrm{D}}{ }^{20}=-26.5\left(c 1.0, \mathrm{CHCl}_{3}\right)$ for an enantiomerically enriched sample of 98:1 e.r., which corresponds to the enantiomeric purity of the ketone $(\mathbf{4 g})$.

\subsection{2 (1S,2R)-1-(4-Fluorophenyl)-2-methyl-1-phenylbut-3-en-1-ol (15b)}

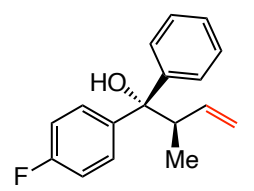

Yellow oil; 23.3 mg, 0.0909 mmol, 91\% yield, 98:2 d.r.; IR (neat): 3547 (br), 3061 (w), 2976 (m), 2932 (w), 1601 (m), 1506 (s), 1446 (m), 1339 (w), 1225 (s), 1160 (s), 1012 (m), 915 (m), 825 (s), 762 (m), 699 (s), 581 (m) cm ${ }^{-1}$; ${ }^{1} \mathbf{H}$ NMR (600 MHz, CDCl $): \delta 7.52-7.46(\mathrm{~m}, 4 \mathrm{H})$, $7.38-7.27$ (m, 2H), 7.23-7.15 (m, 1H), 7.00-6.95 (m, 2H), 5.84 (ddd, $J=17.1,10.6,6.2 \mathrm{~Hz}$, 1H), 5.20-5.12 (m, 2H), 3.52-3.45 (m, 1H), 2.27 (s, 1H), 0.99 (d, $J=6.8 \mathrm{~Hz}, 3 \mathrm{H}) ;{ }^{13} \mathbf{C} \mathbf{N M R}$ (151 MHz, $\left.\mathbf{C D C l}_{3}\right): \delta 161.6(\mathrm{~d}, J=245.3 \mathrm{~Hz}), 145.7,142.6(\mathrm{~d}, J=3.4 \mathrm{~Hz}), 139.1,128.3,127.8$ $(\mathrm{d}, J=8.0 \mathrm{~Hz}), 126.7,125.7,117.6,115.0(\mathrm{~d}, J=21.4 \mathrm{~Hz}), 79.1,44.6,13.7 ;{ }^{19} \mathbf{F}$ NMR (376 MHz, $\mathbf{C D C l}_{3}$ ): $\delta-116.72$ (tt, $J=8.4,5.1 \mathrm{~Hz}$ ); HRMS (DART): Calcd for $\mathrm{C}_{17} \mathrm{H}_{16} \mathrm{~F}[\mathrm{M}+\mathrm{H}-$ $\left.\mathrm{H}_{2} \mathrm{O}\right]^{+}: 239.1231$, Found: 239.1233 ; specific rotation: $[\alpha]_{D}{ }^{20}=-10.3\left(c 1.0, \mathrm{CHCl}_{3}\right)$ for an enantiomerically enriched sample of 99:1 e.r., which corresponds to the enantiomeric purity of the starting alcohol $(\boldsymbol{S}, \boldsymbol{R})-\mathbf{8 d}$.

\subsection{Deprotection of $\alpha$-OPMB ketones}<smiles>C=C(Br)C(Br)(Br)OC(=O)CBr</smiles>

$6 \mathbf{f}$<smiles>C=C(Br)C(O)C(=O)Br</smiles>

16

A vial $(4 \mathrm{~mL}, 17 \times 38 \mathrm{~mm})$ containing a stir bar, was charged with ketone $\mathbf{6 f}(40.1 \mathrm{mg}, 0.10$ $\mathrm{mmol}), \mathrm{CH}_{2} \mathrm{Cl}_{2}(2.0 \mathrm{~mL})$ and $\mathrm{H}_{2} \mathrm{O}(0.1 \mathrm{~mL})$. After 1 minute of gentle stirring, 2,3-dichloro-5,6dicyano-1,4-benzoquinone (DDQ) $(34.1 \mathrm{mg}, 0.15 \mathrm{mmol})$ was added slowly. The vial was allowed to stir for $4 \mathrm{~h}$ at $22{ }^{\circ} \mathrm{C}$, after which the reaction was quenched by the addition of 1.0 $\mathrm{mL}$ of a saturated solution of aqueous $\mathrm{NaHCO}_{3}(1 \mathrm{~mL})$ followed by a saturated solution of 
aqueous $\mathrm{Na}_{2} \mathrm{~S}_{2} \mathrm{O}_{3}(1 \mathrm{~mL})$. The mixture was allowed to stir vigorously for $5 \mathrm{~min}$ at room temperature, after which it was washed with $\mathrm{CH}_{2} \mathrm{Cl}_{2}(3 \times 10 \mathrm{~mL})$ and the volatiles were removed in vacuo affording yellow oil, which was purified by silica gel chromatography (1:5 EtOAc:hexanes) to afford 16 as light yellow oil (27.2 mg, $0.090 \mathrm{mmol}, 90 \%$ yield).

\subsection{1 (R)-3-Hydroxy-1-phenyl-4-(4,4,5,5-tetramethyl-1,3,2-dioxaborolan-2-yl)pent-4-} en-2-one (16)

Colorless oil; $27.2 \mathrm{mg}, 0.090$ mmol, 90\% yield, 95.5:4.5 e.r.; IR (neat): 3471 (b), 2975 (m), 1716 (s), 1616 (w), 1314 (s), 1212 (s), 1165 (s), cm ${ }^{-1}$; ${ }^{1}$ H NMR (600 MHz, CDCl $)$ : $\delta$ 7.33$7.29(\mathrm{~m}, 2 \mathrm{H}), 7.28-7.24(\mathrm{~m}, 1 \mathrm{H}), 7.21-7.17(\mathrm{~m}, 2 \mathrm{H}), 6.16(\mathrm{~d}, J=2.7 \mathrm{~Hz}, 1 \mathrm{H}), 6.04(\mathrm{~d}, J=2.7$ $\mathrm{Hz}, 1 \mathrm{H}), 4.81$ (d, $J=5.6 \mathrm{~Hz}, 1 \mathrm{H}), 3.89$ (d, $J=5.6 \mathrm{~Hz}, 1 \mathrm{H}), 3.82-3.77$ (m, 2H), 1.27 (s, 6H), 1.25 (s, 6H); ${ }^{13} \mathbf{C}$ NMR (151 MHz, $\left.\mathbf{C D C l}_{3}\right): \delta 207.0,134.7,133.5,129.7,128.7,127.2,84.2$, $79.9,77.4,77.2,76.9,44.7,24.9$. The carbon bearing the boron atom could not be detected due to quadrupolar effects; ${ }^{11} \mathbf{B}$ NMR (128 $\left.\mathbf{~ M H z , ~} \mathbf{C D C l}_{3}\right)$ : $\delta$ 30.3; HRMS (DART): Calcd for $\mathrm{C}_{25} \mathrm{H}_{31} \mathrm{BO}_{5}[\mathrm{M}+\mathrm{H}]^{+}:$303.1762, Found: 303.1770 ; specific rotation: $[\alpha]_{\mathrm{D}}{ }^{20}=-18.7$ (c 1.0, $\mathrm{CHCl}_{3}$ ) for an enantiomerically enriched sample of 97.5:2.5 e.r.

Enantiomeric purity was determined by HPLC analysis in comparison with authentic racemic material; Chiralcel IC-3 column, 95.0:5.0 hexanes $/ i-\mathrm{PrOH}, 0.5 \mathrm{~mL} / \mathrm{min}, 220 \mathrm{~nm}$.

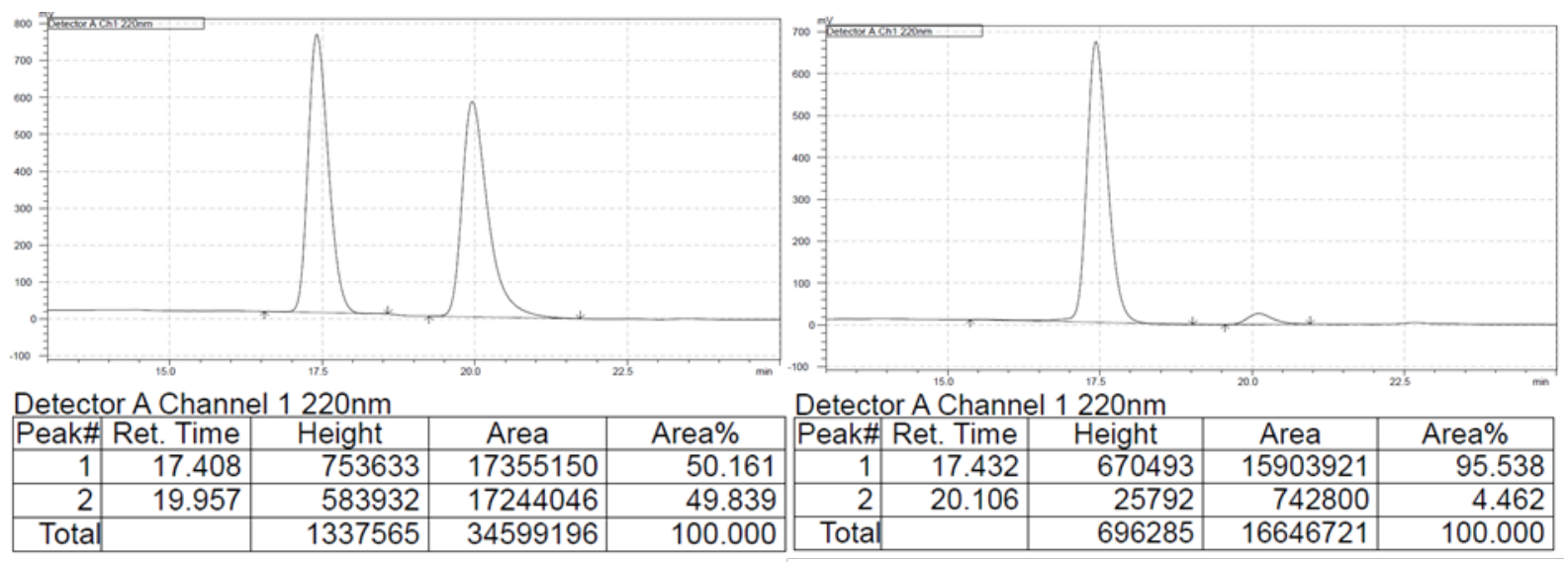

\begin{tabular}{|c|c|c|c|c|c|}
\hline Retention Time & Area & Area\% & Retention Time & Area & Area\% \\
\hline 17.408 & 17355150 & 50.146 & 17.432 & 15903921 & 95.539 \\
\hline 19.957 & 17244046 & 49.839 & 20.106 & 742800 & 4.462 \\
\hline
\end{tabular}

\subsection{Catalytic cross-coupling}

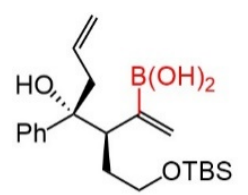

$R, R-8$ a

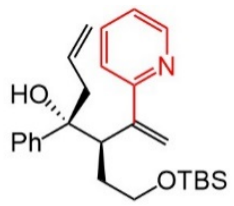

S2

A vial ( $4 \mathrm{~mL}, 17 \times 38 \mathrm{~mm})$ containing a magnetic stir bar was charged with $(\boldsymbol{R}, \boldsymbol{R})-\mathbf{8 a}(55.3 \mathrm{mg}$, $0.153 \mathrm{mmol}$ ), 2-bromopyridine (36.3 $\mathrm{mg}, 0.230 \mathrm{mmol}), \mathrm{Pd}_{2}(\mathrm{dba})_{3}(2.7 \mathrm{mg}, 0.0030 \mathrm{mmol})$ and SPhos (2.5 mg, $0.0060 \mathrm{mmol})$. The vial was sealed (screw cap) and, after THF (1.2 mL) and a 
solution of $3 \mathrm{M} \mathrm{NaOH}(0.4 \mathrm{~mL})$ were added, the mixture was allowed to heat to $60{ }^{\circ} \mathrm{C}$. After 6 $\mathrm{h}$, the mixture was allowed to cool to room temperature, the solution was washed with EtOAc $(3 \times 5 \mathrm{~mL})$, dried over $\mathrm{MgSO}_{4}$, filtered, and the volatiles were removed in vacuo to afford yellowish oil , which was purified by silica gel chromatography (50:1 $\rightarrow$ 10:1hexanes:EtOAc; $\mathrm{R}_{f}=0.25$ in 10:1 hexanes:EtOAc) afforded $\mathbf{S 2}$ as yellow oil (44.1 $\mathrm{mg}, 0.104 \mathrm{mmol}, 68 \%$ yield).

\subsection{1 (3R,4R)-3-(2-((t-Butyldimethylsilyl)oxy)ethyl)-4-phenyl-2-(pyridin-2-yl)hepta-1,6- dien-4-ol (S5)}

Yellow oil; 44.1 mg, 0.104 mmol, 68\% yield, >98:2 d.r.; IR (neat): 3146 (br), 3081 (m), 2949 (m), 2854 (m), 1590 (m), 1470 (m), 1253 (m), 1094 (s), 835 (s), 804 (m), 775 (m) cm ${ }^{-1} ;{ }^{1} \mathbf{H}$

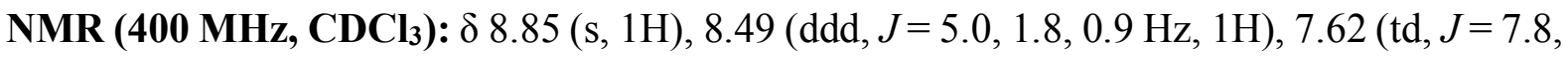
$1.8 \mathrm{~Hz}, 1 \mathrm{H}), 7.43-7.32(\mathrm{~m}, 3 \mathrm{H}), 7.24-7.13(\mathrm{~m}, 3 \mathrm{H}), 7.12-7.04(\mathrm{~m}, 1 \mathrm{H}), 5.68-5.54(\mathrm{~m}, 1 \mathrm{H})$, $5.26(\mathrm{~d}, J=1.1 \mathrm{~Hz}, 1 \mathrm{H}), 5.08-4.98(\mathrm{~m}, 1 \mathrm{H}), 4.96-4.89(\mathrm{~m}, 1 \mathrm{H}), 4.88(\mathrm{~s}, 1 \mathrm{H}), 3.54-3.33(\mathrm{~m}$, $2 \mathrm{H}), 3.18(\mathrm{dd}, J=11.4,2.8 \mathrm{~Hz}, 1 \mathrm{H}), 3.00-2.64(\mathrm{~m}, 2 \mathrm{H}), 2.24-2.11(\mathrm{~m}, 1 \mathrm{H}), 1.82-1.66(\mathrm{~m}, 1 \mathrm{H})$, $0.86(\mathrm{~s}, 9 \mathrm{H}),-0.05$ (s, 3H), -0.06 (s, 3H); ${ }^{13} \mathbf{C}$ NMR (101 MHz, $\left.\mathbf{C D C l}_{3}\right): \delta 158.0,147.4,147.2$, 146.6, 137.7, 135.4, 127.4, 126.4, 125.6, 123.3, 122.9, 122.2, 116.8, 61.6, 54.7, 45.3, 30.2, 26.1, 18.4, -5.2, -5.3; HRMS (ESI): Calcd for $\mathrm{C}_{26} \mathrm{H}_{38} \mathrm{NO}_{2} \mathrm{Si}[\mathrm{M}+\mathrm{H}]^{+}:$424.2666, Found: 424.2665; specific rotation: $[\alpha]_{\mathrm{D}}{ }^{20}=+78.8\left(c 1.0, \mathrm{CHCl}_{3}\right)$ for an enantiomerically enriched sample of $>99: 1$ e.r., which corresponds to the enantiomeric purity of $(\boldsymbol{R}, \boldsymbol{R})-\mathbf{8 a}$.

\subsection{Directed ketone reduction}

A previously reported protocol was used ${ }^{35}$.

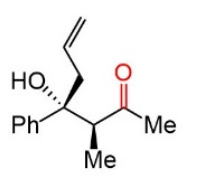

s6

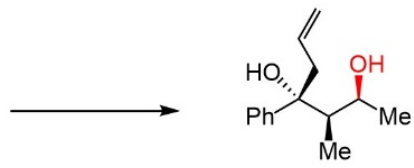

s7

A vial that contained $\mathrm{Me}_{4} \mathrm{~N}(\mathrm{AcO})_{3} \mathrm{BH}(536 \mathrm{mg}, 1.83 \mathrm{mmol})$ and $1: 1$ mixture of $\mathrm{HOAc}: \mathrm{CH}_{3} \mathrm{CN}$ mixture $(1 \mathrm{~mL})$ was charged, at $0{ }^{\circ} \mathrm{C}$ and in a dropwise manner, with a solution of $(3 S, 4 R)-4$ hydroxy-3-methyl-4-phenylhept-6-en-2-one (S7; $40.0 \mathrm{mg} 0.183 \mathrm{mmol})$ dissolved in 1:1 HOAc: $\mathrm{CH}_{3} \mathrm{CN}(2 \mathrm{~mL})$. The mixture was allowed to stir for $3 \mathrm{~h}$ at $0{ }^{\circ} \mathrm{C}$, after which the reaction was quenched by the addition of a saturated solution of aqueous sodium-potassium tartrate ( 5 $\mathrm{mL})$. The mixture was allowed to warm to ambient temperature, after which $\mathrm{CH}_{2} \mathrm{Cl}_{2}(20 \mathrm{~mL})$ was added. It was then washed with an aqueous solution of saturated $\mathrm{NaHCO}_{3}$. The aqueous layer was back-washed with $\mathrm{CH}_{2} \mathrm{Cl}_{2}$ and the combined organic layers were washed with an aqueous solution of saturated $\mathrm{NaHCO}_{3}$. The aqueous layer was back-washed with $\mathrm{CH}_{2} \mathrm{Cl}_{2}(4 \times$ $20 \mathrm{~mL}$ ), and the combined organic layers were dried over anhydrous $\mathrm{Na}_{2} \mathrm{SO}_{3}$ and the volatiles were removed in vacuo, affording colorless oil, which was purified by silica gel chromatography $\left(\mathrm{R}_{f}=0.18,3: 1\right.$ hexanes/Et $\left.2 \mathrm{O}\right)$ to afford the $\beta$-hydroxy ketone $\mathbf{S} 7$ as colorless oil (38.0 mg, $0.172 \mathrm{mmol}, 94 \%$ yield). Diastereomeric purity was determined by analysis of its ${ }^{1} \mathrm{H}$ NMR spectrum $\left(\mathrm{CDCl}_{3} ; 97: 3\right.$ d.r.). 


\subsection{1 (2S,3R,4R)-3-Methyl-4-phenylhept-6-ene-2,4-diol (S7)}

Colorless oil; $38.0 \mathrm{mg}, 0.172 \mathrm{mmol}, 94 \%$ yield, $97: 3$ d.r. determined by ${ }^{1} \mathrm{H} \mathrm{NMR}$ in $\mathrm{CDCl}_{3}$; IR (neat): 3346 (br), 2977 (m), 2917 (w), 1445 (m), 1149 (m), 704 (s) cm ${ }^{-1}$; ${ }^{1}$ H NMR (400 MHz, CDCl$\left._{3}\right): \delta 7.40-7.29(\mathrm{~m}, 4 \mathrm{H}), 7.25-7.21(\mathrm{~m}, 1 \mathrm{H}), 5.41-5.24(\mathrm{~m}, 1 \mathrm{H}), 5.12-4.95(\mathrm{~m}, 2 \mathrm{H}), 3.70$ $(\mathrm{qd}, J=6.4,1.4 \mathrm{~Hz}, 1 \mathrm{H}), 2.87(\mathrm{~s}, 1 \mathrm{H}), 2.70-2.53(\mathrm{~m}, 2 \mathrm{H}), 1.84(\mathrm{qd}, J=7.0,1.4 \mathrm{~Hz}, 1 \mathrm{H}), 1.18$ $(\mathrm{d}, J=7.0 \mathrm{~Hz}, 3 \mathrm{H}), 1.01(\mathrm{~d}, J=6.4 \mathrm{~Hz}, 3 \mathrm{H}) ;{ }^{13} \mathbf{C}$ NMR (101 MHz, CDCl $\left.\mathbf{3}\right): \delta 146.28,133.60$, 128.32, 126.58, 125.54, 119.30, 80.03, 77.48, 77.16, 76.84, 67.68, 46.55, 45.36, 21.68, 6.28; HRMS (ESI): Calcd for $\mathrm{C}_{14} \mathrm{H}_{19} \mathrm{O}\left[\mathrm{M}+\mathrm{H}-\mathrm{H}_{2} \mathrm{O}\right]^{+}$: 203.1430 Found: 203.1425; specific rotation: $[\alpha]_{\mathrm{D}}{ }^{20}=-17.2\left(c 1.0, \mathrm{CHCl}_{3}\right)$ for an enantiomerically enriched sample of 99:1 e.r., which corresponds to the enantiomeric purity of the starting ketone $\mathbf{S 6}$.

\section{Synthesis of the Bicyclic Fragments of (+)-Rubriflordilactone A and B, and 5-epi-Rubriflordilactone $A$ and $B$}

\subsection{Synthesis of the Bicyclic Fragments of (+)-Rubriflordilactone A and B}
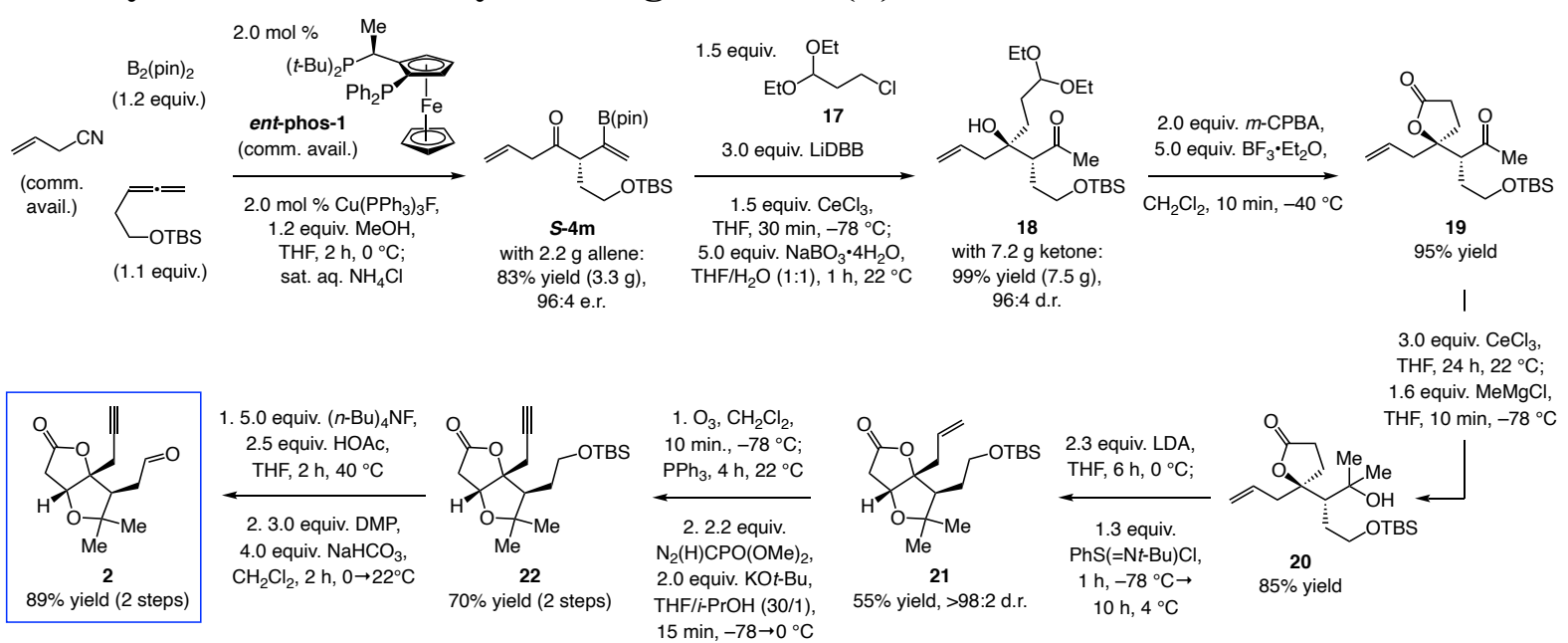

10.1.1 $(S)-3-(2-((t$-Butyldimethylsilyl)oxy)ethyl)-2-(4,4,5,5-tetramethyl-1,3,2-

\section{dioxaborolan-2-yl)hepta-1,6-dien-4-one $(S-4 m)$}

A $100 \mathrm{~mL}$ round-bottom flask containing a stir bar was charged with $\mathrm{CuF}\left(\mathrm{PPh}_{3}\right)_{3} \cdot 2 \mathrm{EtOH}$ (192.3 mg, $0.20 \mathrm{mmol}$ ) and ent-phos-1 (119.4 mg, $0.22 \mathrm{mmol})$; the mixture was purged with $\mathrm{N}_{2}$ (Schlenk line) and then THF was added (20 mL). Anhydrous MeOH (368 mg, $\left.11.5 \mathrm{mmol}\right)$ was then added, after which the solution was allowed to stir at $22{ }^{\circ} \mathrm{C}$ for $10 \mathrm{~min}$. A separate $250 \mathrm{~mL}$ round-bottom flask containing $\mathrm{B}_{2}(\text { pin })_{2}(3.05 \mathrm{~g}, 1.2 \mathrm{mmol})$ was purged with $\mathrm{N}_{2}$ and allyl cyanide $(670 \mathrm{mg}, 10.0 \mathrm{mmol}), t$-butyldimethyl(penta-3,4-dien-1-yloxy)silane (2.18 g, $1.10 \mathrm{mmol})$, and THF (60 mL) were added. The resulting solution was allowed to stir for $2 \mathrm{~h}$ at $0{ }^{\circ} \mathrm{C}$. The solution was allowed to cool to $0{ }^{\circ} \mathrm{C}$ and then it was added the solution of bisphosphine- $\mathrm{Cu}$ catalyst through canula (within $\sim 1 \mathrm{~min}$ ). The mixture was allowed to stir for $2 \mathrm{~h}$ at $0{ }^{\circ} \mathrm{C}$. After complete conversion was observed (monitored by TLC), the reaction was quenched by the addition of a saturated solution of aqueous $\mathrm{NH}_{4} \mathrm{Cl}(50 \mathrm{~mL})$. The mixture was diluted with water $(50 \mathrm{~mL})$ and washed with $\mathrm{Et}_{2} \mathrm{O}(3 \times 40 \mathrm{~mL})$. The combined organic layers 
were dried over $\mathrm{Na}_{2} \mathrm{SO}_{4}$, filtered, and concentrated in vacuo to afford pale-yellow oil, which was purified by silica gel chromatography $\left(100: 2 \rightarrow 100: 3\right.$ hexanes:EtOAc; $\mathrm{R}_{f}=0.40$ (5:1 hexanes:EtOAc)) to afford $S-\mathbf{4 m}$ as colorless oil (3.28 g, $8.3 \mathrm{mmol}, 83 \%$ yield).

Colorless oil; 3.28 g, 8.3 mmol, 83\% yield, 96:4 e.r.; IR (neat): 2926 (w), 2854 (w), 1712 (m), 1312 (m), 1251 (m), 1139 (s), 1096 (m), 833 (s), 774 (s) cm ${ }^{-1}$; ${ }^{\mathbf{1}} \mathbf{H}$ NMR (CDCl $\mathbf{3}, 500$ MHz): $\delta 5.96(\mathrm{~d}, J=2.7 \mathrm{~Hz}, 1 \mathrm{H}), 5.90(\mathrm{ddt}, J=17.2,10.2,6.9 \mathrm{~Hz}, 1 \mathrm{H}), 5.66(\mathrm{~d}, J=3.2 \mathrm{~Hz}, 1 \mathrm{H}), 5.15-$ $5.02(\mathrm{~m}, 2 \mathrm{H}), 3.60-3.49$ (m, 3H), 3.20 (ddt, $J=7.2,4.5,1.4 \mathrm{~Hz}, 2 \mathrm{H}), 2.06$ (dq, $J=13.5,6.8$ $\mathrm{Hz}, 1 \mathrm{H}), 1.70$ (ddt, $J=13.3,7.2,6.0 \mathrm{~Hz}, 1 \mathrm{H}), 1.25$ (s, 12H), 0.87 (s, 9H), 0.01 (s, 3H), 0.01 (s, $3 \mathrm{H}) ;{ }^{13} \mathbf{C}$ NMR (CDCl 3,101 MHz): $\delta 208.8,132.3,131.3,118.3,83.9,61.0,53.1,46.7,33.6$, 26.1, 24.88, 24.85, 18.4, -5.18, -5.20; HRMS (DART): Calcd for $\mathrm{C}_{21} \mathrm{H}_{40} \mathrm{BO}_{4} \mathrm{Si}[\mathrm{M}+\mathrm{H}]^{+}$: 395.2783, Found: 395.2778; specific rotation: $\left.[\alpha]_{\mathrm{D}}{ }^{20}=+59.2(c) 1.5, \mathrm{CHCl}_{3}\right)$ for an enantiomerically enriched sample of 96:4 e.r.
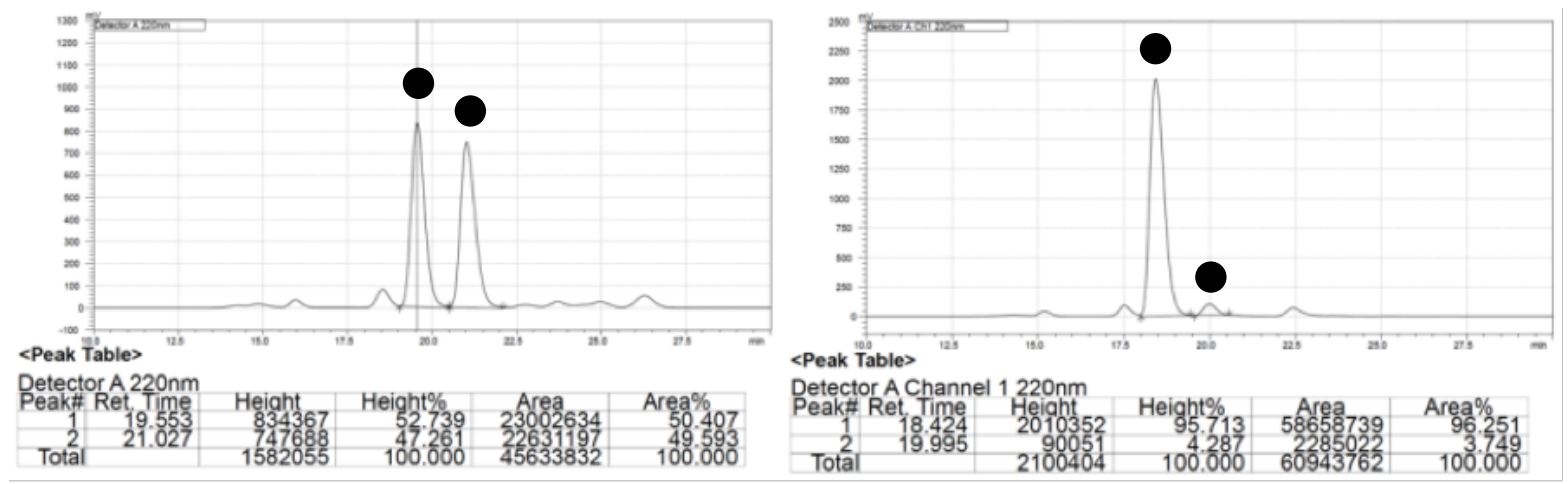

\begin{tabular}{|c|c|c|c|c|c|}
\hline Retention Time & Area & Area\% & Retention Time & Area & Area\% \\
\hline 19.553 & 23002634 & 50.407 & 18.424 & 58658739 & 96.251 \\
\hline 21.027 & 22631197 & 49.593 & 19.995 & 2285022 & 3.749 \\
\hline
\end{tabular}

10.1.2 (3R,4R)-3-(2-((t-Butyldimethylsilyl)oxy)ethyl)-4-(3,3-diethoxypropyl)-4hydroxyhept-6-en-2-one (18)

A $500 \mathrm{~mL}$ round-bottom flask with a stir bar was purged with $\mathrm{N}_{2}$ (Schlenk line) and then charged with 3-chloropropionaldehyde diethylacetal (17; $4.53 \mathrm{~g}, 27.2 \mathrm{mmol})$ and THF (30 mL). The solution was allowed to cool to $-78{ }^{\circ} \mathrm{C}$ after which it was charged with lithium di-tbutylbiphenylide (LiDBB; $133 \mathrm{~mL}, 0.41 \mathrm{M}$ in THF) by syringe. This deep-green solution was allowed to stir for $1 \mathrm{~h}$ at $-78{ }^{\circ} \mathrm{C}$ after which it was transferred by cannula to a $1000 \mathrm{~mL}$ roundbottom flask that contained a previously cooled $\left(-78^{\circ} \mathrm{C}\right)$ suspension of $\mathrm{CeCl}_{3}(6.69 \mathrm{~g}, 27.2$ $\mathrm{mmol})$ in THF $(100 \mathrm{~mL})$. The resulting cloudy red mixture was allowed to stir for $1 \mathrm{~h} \mathrm{at}-78$ ${ }^{\circ} \mathrm{C}$ for $1 \mathrm{~h}$ and then a solution of ketone $\boldsymbol{S}-\mathbf{4 m}(7.19 \mathrm{~g}, 18.2 \mathrm{mmol})$ in THF (30 mL) was added through cannula. The mixture was allowed to stir for $30 \mathrm{~min}$ at $-78^{\circ} \mathrm{C}$, after which the reaction was quenched by the addition of a saturated solution aqueous potassium sodium tartrate (150 $\mathrm{mL})$ and water $(150 \mathrm{~mL})$. The solution was allowed to become clear and then it was charged with sodium perborate tetrahydrate $(14.0 \mathrm{~g}, 91.0 \mathrm{mmol})$ and the mixture was allowed to stir for $1 \mathrm{~h}$ at $22{ }^{\circ} \mathrm{C}$ after which EtOAc $(300 \mathrm{~mL})$ and water $(300 \mathrm{~mL})$ were added. The mixture was washed with EtOAc $(3 \times 150 \mathrm{~mL})$ and the combined organic layers were washed with brine 
$(200 \mathrm{~mL})$, dried over $\mathrm{Na}_{2} \mathrm{SO}_{4}$, and the volatiles were removed in vacuo to afford yellow solid. Purification by column chromatography $\left(20: 1 \rightarrow 5: 1\right.$ hexanes:EtOAc; $\mathrm{R}_{f}=0.45(2: 1$ hexanes:EtOAc)) afforded tertiary alcohol 18 as colorless oil (7.50 g, $18.0 \mathrm{mmol}, 99 \%$ yield).

Colorless oil; 7.50 g, 18.0 mmol, 99\% yield, 96:4 d.r., 96:4 e.r.; IR (neat): 3474 (w), 2927 (m), 2855 (m), 1696 (s), 1372 (w), 1254 (m), 1092 (s), 938 (m), 834 (s), 776 (m) cm ${ }^{-1} ;{ }^{1} \mathbf{H}$ NMR (CDCl, 500 MHz): $\delta 5.86$ (ddt, $J=17.2,10.3,7.3 \mathrm{~Hz}, 1 \mathrm{H}), 5.15-5.04(\mathrm{~m}, 2 \mathrm{H}), 4.45-4.40$ (m, $1 \mathrm{H}), 3.68-3.58(\mathrm{~m}, 3 \mathrm{H}), 3.56-3.42(\mathrm{~m}, 4 \mathrm{H}), 2.95(\mathrm{dd}, J=10.0,3.5 \mathrm{~Hz}, 1 \mathrm{H}), 2.29-2.17(\mathrm{~m}$, $5 \mathrm{H}), 1.93-1.74(\mathrm{~m}, 2 \mathrm{H}), 1.72-1.53(\mathrm{~m}, 3 \mathrm{H}), 1.51-1.42(\mathrm{~m}, 1 \mathrm{H}), 1.19(\mathrm{td}, J=7.1,1.6 \mathrm{~Hz}, 6 \mathrm{H})$,

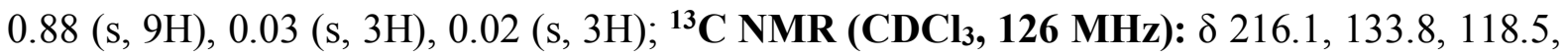
103.4, 75.1, 61.61, 61.55, 61.47, 54.0, 43.1, 34.1, 31.0, 30.6, 27.6, 26.0, 18.4, 15.5, 15.4, -5.3, -5.4; HRMS (DART): Calcd for $\mathrm{C}_{22} \mathrm{H}_{44} \mathrm{O}_{5} \mathrm{Si}[\mathrm{M}+\mathrm{H}]^{+}:$417.3031, Found: 417.3003; specific rotation: $[\alpha]_{\mathrm{D}}{ }^{20}=-11.4\left(c 0.55, \mathrm{CHCl}_{3}\right)$.

We determined the enantiomeric purity of $\mathbf{1 8}$ by HPLC analysis of the pyridyl derivative $\mathbf{S 8}$. The presence of a boronic acid group complicates HPLC analysis (significant peak broadening). Chiralcel AZ-H column, 95:5 hexanes $/ i-\mathrm{PrOH}, 0.5 \mathrm{~mL} / \mathrm{min}, 254 \mathrm{~nm}$.

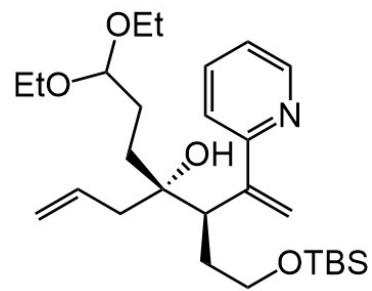

S8
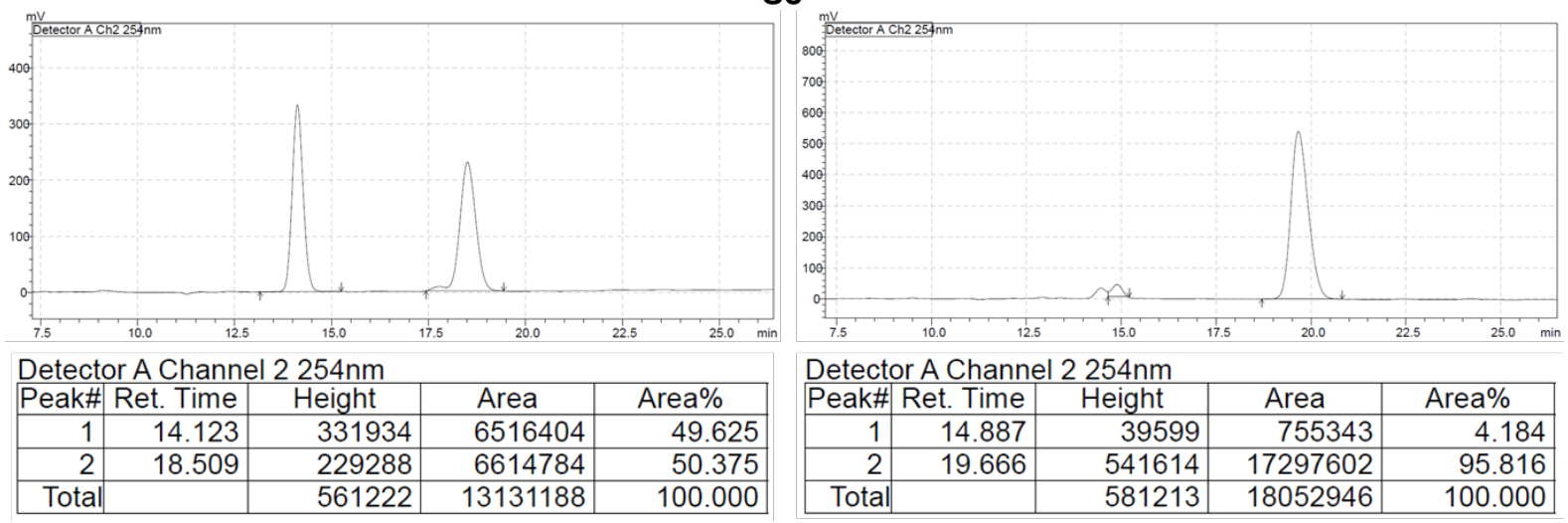

\begin{tabular}{|c|c|c|c|c|}
\hline \multicolumn{5}{|c|}{ Detector A Channel 2 254nm } \\
\hline Peak\# & Ret. Time & Height & Area & Area\% \\
\hline 1 & 14.123 & 331934 & 6516404 & 49.625 \\
\hline 2 & 18.509 & 229288 & 6614784 & 50.375 \\
\hline Total & & 561222 & 13131188 & 100.000 \\
\hline
\end{tabular}

\begin{tabular}{|c|c|c|c|c|}
\hline \multicolumn{5}{|c|}{ nel 2 254nm } \\
\hline Peak & Ret. Time & Height & Area & Area\% \\
\hline 1 & 14.887 & 39599 & 755343 & 4.184 \\
\hline 2 & 19.666 & 541 & 172976 & 95.816 \\
\hline Tota & & 581213 & 18052946 & 100.000 \\
\hline
\end{tabular}

\begin{tabular}{|c|c|c|c|c|c|}
\hline Retention Time & Area & Area $\%$ & Retention Time & Area & Area\% \\
\hline 14.123 & 6516404 & 49.625 & 14.887 & 755343 & 4.184 \\
\hline 18.509 & 6614784 & 50.375 & 19.666 & 17297602 & 95.816 \\
\hline
\end{tabular}

10.1.3 $(R)$-5-Allyl-5-((R)-1-((t-butyldimethylsilyl)oxy)-4-oxopentan-3-yl)dihydrofuran2(3H)-one (19)

A solution of acetal $18(4.00 \mathrm{~g}, 9.6 \mathrm{mmol})$ in $\mathrm{CH}_{2} \mathrm{Cl}_{2}(100 \mathrm{~mL})$ in a $1000 \mathrm{~mL}$ round-bottom flask that contained a stir bar, was allowed to cool at $-40{ }^{\circ} \mathrm{C}$. The solution was charged with $m$-CPBA (75\% pure reagent, $4.42 \mathrm{~g}, 19.2 \mathrm{mmol}$ ), and the resulting cloudy mixture was allowed to stir for $10 \mathrm{~min}$ at $-40{ }^{\circ} \mathrm{C}$. In a separate $250 \mathrm{~mL}$ round-bottom flask, a solution of $\mathrm{BF}_{3} \cdot \mathrm{OEt}_{2}$ (6.0 mL, $48 \mathrm{mmol})$ in $\mathrm{CH}_{2} \mathrm{Cl}_{2}(40 \mathrm{~mL})$ was allowed to cool to $-78^{\circ} \mathrm{C}$; this was transferred to 
the aforementioned solution of acetal 18 through canula quickly $(\sim 10 \mathrm{sec})$ while the solution was allowed to stir vigorous. (The $1000 \mathrm{~mL}$ round-bottom flask was placed under vacuum to reduce the time of bulk solution transfer.) The mixture was allowed to stir for $10 \mathrm{~min}$ at -40 ${ }^{\circ} \mathrm{C}$ and reaction progress was monitored by TLC. Once complete conversion was reached, the reaction was quenched by the addition of saturated solution of aqueous $\mathrm{NaHSO}_{3}(100 \mathrm{~mL})$. (Longer reaction time can lead to byproduct formation, including the premature removal of the silyl protecting group.) The aqueous layer was washed by $\mathrm{CH}_{2} \mathrm{Cl}_{2}(3 \times 100 \mathrm{~mL})$ and the combined organic layer was washed by an aqueous solution of $1.0 \mathrm{M} \mathrm{K}_{2} \mathrm{CO}_{3}(100 \mathrm{~mL})$, a saturated solution of $\mathrm{Na}_{2} \mathrm{CO}_{3}(200 \mathrm{~mL})$ and brine $(200 \mathrm{~mL})$. The organic layers were dried over $\mathrm{Na}_{2} \mathrm{SO}_{4}$ and the volatiles were removed in vacuo to afford yellow oil, purification of which by silica gel chromatography $\left(10: 1 \rightarrow 3: 1\right.$ hexanes:EtOAc; $\mathrm{R}_{f}=0.38$ (2:1 hexanes:EtOAc) $)$ afforded lactone 19 as colorless oil (3.10 g, $9.1 \mathrm{mmol}, 95 \%$ yield).

Colorless oil; 3.10 g, 9.1 mmol, 95\% yield; IR (neat): 2927 (m), 2855 (w), 1776 (s), 1711 (m), 1359 (w), 1184 (w), 1092 (m), 929 (w), 834 (s), 777 (m) cm $\left.{ }^{-1} ;{ }^{1} \mathbf{H ~ N M R ~ ( C D C l}_{3}, \mathbf{5 0 0} \mathbf{~ M H z}\right)$ $\delta 5.85$ (ddt, $J=17.4,10.2,7.2 \mathrm{~Hz}, 1 \mathrm{H}), 5.27-5.16(\mathrm{~m}, 2 \mathrm{H}), 3.60$ (dt, $J=10.9,5.6 \mathrm{~Hz}, 1 \mathrm{H})$, $3.54-3.49(\mathrm{~m}, 1 \mathrm{H}), 3.32(\mathrm{dd}, J=11.0,2.6 \mathrm{~Hz}, 1 \mathrm{H}), 2.68-2.59(\mathrm{~m}, 1 \mathrm{H}), 2.52-2.46(\mathrm{~m}, 2 \mathrm{H}), 2.43$ $(\mathrm{d}, J=7.2,2 \mathrm{H}), 1.99-1.89(\mathrm{~m}, 1 \mathrm{H}), 1.85(\mathrm{ddd}, J=12.9,9.2,5.5 \mathrm{~Hz}, 1 \mathrm{H}), 1.60-1.51(\mathrm{~m}, 1 \mathrm{H})$,

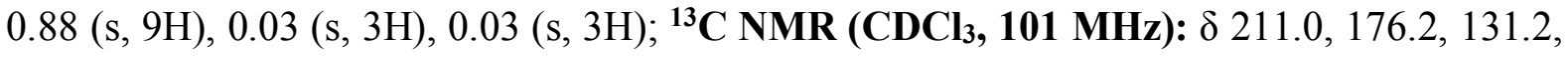
120.9, 88.8, 61.0, 53.7, 43.7, 33.9, 30.9, 28.8, 26.3, 26.0, 18.4, -5.39, -5.44; HRMS (DART): Calcd for $\mathrm{C}_{18} \mathrm{H}_{33} \mathrm{O}_{4} \mathrm{Si}[\mathrm{M}+\mathrm{H}]^{+}: 341.2142$, Found: 341.2150 ; specific rotation: $[\alpha]_{D^{20}}=-55.6$ (c $\left.0.62, \mathrm{CHCl}_{3}\right)$.

\subsection{4 (R)-5-Allyl-5-((S)-1-((t-butyldimethylsilyl)oxy)-4-hydroxy-4-methylpentan-3- yl)dihydrofuran-2(3H)-one (20)}

A $1000 \mathrm{~mL}$ round-bottom flask containing a stir bar was charged at $22{ }^{\circ} \mathrm{C}$ with ketone $19(4.50$ $\mathrm{g}, 13.2 \mathrm{mmol}$ ), $\mathrm{CeCl}_{3}(9.75 \mathrm{~g}, 39.6 \mathrm{mmol})$ and the solution was purged with $\mathrm{N}_{2}$ (Schlenk line), and then THF was added $(200 \mathrm{~mL})$. The mixture was allowed to stir for $24 \mathrm{~h}$ at $22{ }^{\circ} \mathrm{C}$ before being allowed to cool to $-78^{\circ} \mathrm{C}$. In a $100 \mathrm{~mL}$ round-bottom flask, a solution of $\mathrm{MeMgCl}(3.0$ $\mathrm{M}$ in THF, $7.0 \mathrm{~mL}, 21 \mathrm{mmol})$ was diluted in THF ( $35 \mathrm{~mL})$ and the solution was allowed to cool at $-78{ }^{\circ} \mathrm{C}$. The solution of $\mathrm{MeMgCl}$ was then transferred to a vigorously stirrung solution of ketone 19 by cannula at $-78{ }^{\circ} \mathrm{C}$, and the mixture was allowed to stir for $10 \mathrm{~min}$ at $-78{ }^{\circ} \mathrm{C}$. (After $\sim 80 \%$ of the solution was transferred, the reaction was monitored by TLC after each 5.0 $\mathrm{mL}$ solution was added.) After the reaction reached completion (monitored by TLC), it was quenched by the addition of a saturated solution of aqueous potassium sodium tartrate (150 $\mathrm{mL})$ and water $(100 \mathrm{~mL})$. The mixture was allowed to stir for $1 \mathrm{~h}$ at $22{ }^{\circ} \mathrm{C}$, at which time the solution turned clear. The mixture was then washed with EtOAc $(4 \times 150 \mathrm{~mL})$ and the combined organic layers were washed with a saturated solution of aqueous $\mathrm{NaHCO}_{3}(200 \mathrm{~mL})$ and brine $(200 \mathrm{~mL})$ and then dried over a bed of $\mathrm{Na}_{2} \mathrm{SO}_{4}$. Filtration and removal of the volatiles in vacuo afforded pale-yellow oil, which was purified by silica gel chromatography $(5: 1 \rightarrow 2: 1$ hexanes:EtOAc; $\mathrm{R}_{f}=0.25$ (2:1 Hexanes:EtOAc)) to afford alcohol 20 as colorless oil (3.97 g, $11.2 \mathrm{mmol}, 85 \%$ yield). 
Colorless oil; 3.97 g, 11.2 mmol, 85\% yield; IR (neat): 3443 (w), 2953 (m), 2926 (s), 2854 (m), 1759 (s), 1470 (w), 1253 (m), 1201 (m), 1091 (s), 1005 (w), 921 (w), 835 (s), 776 (m) $\mathrm{cm}^{-1} ;{ }^{1} \mathbf{H}$ NMR $\left(\mathbf{C D C l}_{3}, \mathbf{4 0 0} \mathbf{M H z}\right): \delta 5.82(\mathrm{ddt}, J=17.3,10.5,7.2 \mathrm{~Hz}, 1 \mathrm{H}), 5.26-5.17(\mathrm{~m}$, $2 \mathrm{H}), 3.76-3.62(\mathrm{~m}, 2 \mathrm{H}), 2.92(\mathrm{~s}, 1 \mathrm{H}), 2.64-2.34(\mathrm{~m}, 5 \mathrm{H}), 2.16-2.04(\mathrm{~m}, 1 \mathrm{H}), 2.03(\mathrm{dd}, J=5.2$, $3.2 \mathrm{~Hz}, 1 \mathrm{H}), 1.85-1.71(\mathrm{~m}, 1 \mathrm{H}), 1.52$ (ddt, $J=15.1,6.1,2.7 \mathrm{~Hz}, 1 \mathrm{H}), 1.31(\mathrm{~s}, 3 \mathrm{H}), 1.24(\mathrm{~s}, 3 \mathrm{H})$, 0.90 (s, 9H), 0.07 (s, 6H); ${ }^{13} \mathbf{C}$ NMR (CDCl, 126 MHz): $\delta$ 176.7, 131.8, 120.8, 91.1, 73.2, 63.7, 52.6, 44.8, 31.0, 29.7, 29.6, 29.5, 29.0, 26.1 , 18.4, -5.2, -5.3; HRMS (DART): Calcd for $\mathrm{C}_{19} \mathrm{H}_{37} \mathrm{O}_{4} \mathrm{Si}[\mathrm{M}+\mathrm{H}]^{+}: 357.2456$, Found: 357.2475 ; specific rotation: $[\alpha]_{\mathrm{D}}{ }^{20}=-14.8(c 0.32$, $\left.\mathrm{CHCl}_{3}\right)$.

\subsection{5 (3a $R, 6 S, 6 a R)-6 a-A l l y l-6-(2-((t$-butyldimethylsilyl)oxy)ethyl)-5,5- dimethyltetrahydrofuro[3,2-b]furan-2(3H)-one $(21)$}

In a $250 \mathrm{~mL}$ round-bottom flask, alcohol $20(1.18 \mathrm{~g}, 3.31 \mathrm{mmol})$ was dried by azeotropic distillation with anhydrous benzene $\left(3 \times 20 \mathrm{~mL}\right.$ ). The flask was then purged with $\mathrm{N}_{2}$ (Schlenk line) and THF was added $(40 \mathrm{~mL})$. The was allowed to cool to $0{ }^{\circ} \mathrm{C}$ while being allowed to stir at $0{ }^{\circ} \mathrm{C}$ and was charged with lithium diisopropylamide (LDA, $1.5 \mathrm{M}, 5.1 \mathrm{~mL}, 7.6 \mathrm{mmol}$ ). The resulting orange solution was allowed to stir for $6 \mathrm{~h}$ at $0{ }^{\circ} \mathrm{C}$ before being cooled to $-78{ }^{\circ} \mathrm{C}$ and was charged by cannula in $10 \mathrm{sec}$ with a solution of $N$-t-butylbenzenesulfinimidoyl chloride $(0.930 \mathrm{~g}, 4.33 \mathrm{mmol})$ in THF $(5.0 \mathrm{~mL})$ that was cooled to $-78^{\circ} \mathrm{C}$ beforehand. The mixture was allowed to stir for $1 \mathrm{~h}$ at $-78{ }^{\circ} \mathrm{C}$ after which it was allowed to warm to $0{ }^{\circ} \mathrm{C}$ and stir for $12 \mathrm{~h}$ at $0{ }^{\circ} \mathrm{C}$. At this time, the reaction was quenched by the addition of a saturated aqueous solution of $\mathrm{NH}_{4} \mathrm{Cl}(50 \mathrm{~mL})$ after which the mixture was washed with EtOAc $(4 \times 80 \mathrm{~mL})$. The combined organic layers were washed with brine, dried over $\mathrm{Na}_{2} \mathrm{SO}_{4}$ and the volatiles were removed in vacuo to afford yellow oil, purification of which by silica gel chromatography $(10: 1 \rightarrow 5: 1 \rightarrow 3: 1$ hexanes:EtOAc; $\mathrm{R}_{f}=0.48$ (2:1 hexanes:EtOAc) $)$ to afford lactone 21 as yellow oil $(0.651 \mathrm{~g}$, $1.84 \mathrm{mmol}, 55 \%$ yield $)$ and recovered starting material 20 ( $0.217 \mathrm{~g}, 0.61 \mathrm{mmol}, 18 \%$ yield $)$.

Yellow oil; 0.651 g, 1.84 mmol, 55\% yield; IR (neat): 2927 (m), 2855 (w), 1776 (s), 1711 (m), 1359 (w), 1184 (w), 1092 (m), 929 (w), 834 (s), 777 (m) cm ${ }^{-1} ;{ }^{1} \mathbf{H ~ N M R ~ ( C D C l}_{3}, 600$ MHz): $\delta 5.69$ (dddd, $J=17.1,10.2,8.6,6.0 \mathrm{~Hz}, 1 \mathrm{H}), 5.27-5.19$ (m, 2H), 4.29 (dd, $J=6.8,1.3 \mathrm{~Hz}$, $1 \mathrm{H}$ ), 3.72 (ddd, $J=10.2,8.2,5.5 \mathrm{~Hz}, 1 \mathrm{H}), 3.64$ (dt, $J=10.2,7.2 \mathrm{~Hz}, 1 \mathrm{H}$ ), 2.75 (ddt, $J=14.1$, 5.9, $1.4 \mathrm{~Hz}, 1 \mathrm{H}), 2.69(\mathrm{dd}, J=18.8,6.9 \mathrm{~Hz}, 1 \mathrm{H}), 2.59$ (dd, $J=18.8,1.3 \mathrm{~Hz}, 1 \mathrm{H}), 2.25$ (dd, $J=$ $14.2,8.5 \mathrm{~Hz}, 1 \mathrm{H}), 2.13(\mathrm{dd}, J=9.9,4.3 \mathrm{~Hz}, 1 \mathrm{H}), 1.68$ (dddd, $J=14.0,9.9,7.6,5.5 \mathrm{~Hz}, 1 \mathrm{H})$, 1.48 (dddd, $J=13.9,8.2,6.8,4.3 \mathrm{~Hz}, 1 \mathrm{H}), 1.31$ (s, 3H), 1.07 (s, 3H), 0.89 (s, 9H), 0.07 (s, 3H), 0.07 (s, 3H); ${ }^{13} \mathbf{C}$ NMR (CDCl 3 , 126 MHz): $\delta$ 175.9, 130.6, 121.3, 96.4, 83.4, 78.0, 61.8, 55.9, 39.1, 37.5, 29.2, 28.0, 26.1, 20.7, 18.4, -5.18, -5.21; HRMS (DART): Calcd for $\mathrm{C}_{19} \mathrm{H}_{35} \mathrm{O}_{4} \mathrm{Si}$ $[\mathrm{M}+\mathrm{H}]^{+}: 355.2299$, Found: 355.2301 ; specific rotation: $[\alpha]_{\mathrm{D}}{ }^{20}=+28.9\left(c 0.71, \mathrm{CHCl}_{3}\right)$.

\subsection{6 (3aR,6S,6aR)-6-(2-((t-Butyldimethylsilyl)oxy)ethyl)-5,5-dimethyl-6a-(prop-2-yn- 1-yl)tetrahydrofuro[3,2-b]furan-2(3H)-one (22)}

In a $250 \mathrm{~mL}$ round-bottom flask, a solution of alkene $21(1.30 \mathrm{~g}, 3.67 \mathrm{mmol})$ in $\mathrm{CH}_{2} \mathrm{Cl}_{2}(40$ $\mathrm{mL}$ ) was allowed to cool to $-78{ }^{\circ} \mathrm{C}$ after which $\mathrm{O}_{3}$ was introduced into the solution until it turned blue, immediately after which $\mathrm{PPh}_{3}(\sim 0.50 \mathrm{~g}, \sim 1.9 \mathrm{mmol})$ was added until the blue 
solution disappeared; a second portion of $\mathrm{PPh}_{3}(1.93 \mathrm{~g}, 7.34 \mathrm{mmol})$ was added after which the mixture was allowed to warm to $22{ }^{\circ} \mathrm{C}$. The solution was allowed to stir at for $4 \mathrm{~h} 22{ }^{\circ} \mathrm{C}$ and the volatiles were removed in vacuo to afford yellow oil, purification of which through silica gel chromatography $\left(5: 1 \rightarrow 3: 2\right.$ hexane:EtOAc; $\mathrm{R}_{f}=0.20$ (2:1 hexanes:EtOAc)) to afford the desired aldehyde as white solid (1.23 g, $3.46 \mathrm{mmol}, 94 \%$ yield).

White solid; m.p.: $80^{\circ} \mathrm{C}$ (decomp.); $1.23 \mathrm{~g}, 3.46$ mmol, 94\% yield; IR (neat): 2926 (w), 2854 (w), 1774 (s), 1725 (m), 1386 (w), 1253 (m), 1198 (m), 1179 (m), 1092 (s), 1041 (m), 933 (w), 834 (s), 775 (s) cm ${ }^{-1} ;{ }^{1} \mathbf{H}$ NMR (CDCl 3 , 600 MHz): $\delta 9.74(\mathrm{~s}, 1 \mathrm{H}), 4.47$ (dd, $J=7.4,1.5 \mathrm{~Hz}$, 1H), 3.75-3.65 (m, 2H), 3.39 (d, $J=18.4 \mathrm{~Hz}, 1 \mathrm{H}), 3.26$ (dd, $J=18.6,7.3 \mathrm{~Hz}, 1 \mathrm{H}), 2.76$ (d, $J$ $=18.4 \mathrm{~Hz}, 1 \mathrm{H}), 2.64(\mathrm{dd}, J=18.6,1.4 \mathrm{~Hz}, 1 \mathrm{H}), 2.09(\mathrm{dd}, J=10.1,4.2 \mathrm{~Hz}, 1 \mathrm{H}), 1.63$ (dddd, $J$ $=12.9,10.0,8.6,6.1 \mathrm{~Hz}, 1 \mathrm{H}), 1.54-1.48(\mathrm{~m}, 1 \mathrm{H}), 1.31(\mathrm{~s}, 3 \mathrm{H}), 1.03(\mathrm{~s}, 3 \mathrm{H}), 0.90(\mathrm{~s}, 9 \mathrm{H}), 0.08$ (s, 3H), 0.08 (s, 3H) ${ }^{13}{ }^{\mathbf{C}}$ NMR (CDCl $\mathbf{3}, 151$ MHz): $\delta$ 197.9, 175.9, 92.8, 83.0, 78.9, 61.7, 57.0, 48.1, 38.2, 29.3, 28.0, 26.1, 20.6, 18.4, -5.2, -5.3; HRMS (DART): Calcd for $\mathrm{C}_{18} \mathrm{H}_{33} \mathrm{O}_{5} \mathrm{Si}$ $[\mathrm{M}+\mathrm{H}]^{+}: 357.2092$, Found: 357.2088 ; specific rotation: $[\alpha]_{\mathrm{D}}{ }^{20}=+17.2\left(c 1.0, \mathrm{CHCl}_{3}\right)$.

A $250 \mathrm{~mL}$ round-bottom flask containing a stir bar and $\mathrm{KOt}$-Bu $(771 \mathrm{mg}, 6.88 \mathrm{mmol})$, was purged with $\mathrm{N}_{2}$ (Schlenk line). To this was added $i$-PrOH $(1.5 \mathrm{~mL})$ and THF $(6.0 \mathrm{~mL})$ and the solution was allowed to cool to $-78{ }^{\circ} \mathrm{C}$ after which it was charged with a solution of the $\mathrm{N}_{2}(\mathrm{H}) \mathrm{CPO}(\mathrm{OMe})_{2}$ (the Seyforth-Gilbert reagent; $1.14 \mathrm{~g}, 7.57 \mathrm{mmol}$, in $4.0 \mathrm{~mL}$ THF) dropwise. The mixture was allowed to stir for $10 \mathrm{~min}$ at $-78{ }^{\circ} \mathrm{C}$ after which a solution of the abovementioned aldehyde was added ( $1.23 \mathrm{~g}, 3.44 \mathrm{mmol}$, in $35 \mathrm{~mL}$ THF) through cannula. The solution was then allowed to warm to $0{ }^{\circ} \mathrm{C}(\sim 15 \mathrm{~min})$. After complete consumption of the aldehyde was observed (according to TLC analysis), the reaction was quenched by addition of a saturated solution of aqueous $\mathrm{NH}_{4} \mathrm{Cl}(50 \mathrm{~mL})$. The mixture was washed with EtOAc $(3 \times 50$ $\mathrm{mL}$ ), after which the combined organic layers were washed with brine, dried over $\mathrm{Na}_{2} \mathrm{SO}_{4}$ and the volatiles were removed in vacuo to afford yellow oil, purification of which through silica gel chromatography $\left(15: 1 \rightarrow 5: 1\right.$ hexanes:EtOAc; $\mathrm{R}_{f}=0.50(2: 1$ hexanes:EtOAc) $)$ to afford 21 as white solid $(0.90 \mathrm{~g}, 2.56 \mathrm{mmol}, 74 \%$ yield $)$.

White solid; m.p.: 50-51 ${ }^{\circ} \mathrm{C}$; 0.90 g, 2.56 mmol, 74\% yield; IR (neat): 2922 (s), 2852 (m), $1787(\mathrm{~s}), 1462(\mathrm{w}), 1385(\mathrm{w}), 1254(\mathrm{w}), 1177(\mathrm{w}), 1100(\mathrm{~m}), 1029(\mathrm{w}), 928(\mathrm{w}), 836(\mathrm{~m}), 776$ (m) $\mathrm{cm}^{-1}$; ; ${ }^{1} \mathbf{H}$ NMR (CDCl 3 , 400 MHz): $\delta 4.49(\mathrm{dd}, J=6.9,1.0 \mathrm{~Hz}, 1 \mathrm{H}), 3.73-3.61(\mathrm{~m}, 2 \mathrm{H})$, $3.00(\mathrm{dd}, J=18.7,7.0 \mathrm{~Hz}, 1 \mathrm{H}), 2.90(\mathrm{dd}, J=17.0,2.6 \mathrm{~Hz}, 1 \mathrm{H}), 2.66(\mathrm{dd}, J=18.7,1.1 \mathrm{~Hz}, 1 \mathrm{H})$, $2.49(\mathrm{dd}, J=17.0,2.6 \mathrm{~Hz}, 1 \mathrm{H}), 2.17(\mathrm{dd}, J=9.8,4.4 \mathrm{~Hz}, 1 \mathrm{H}), 2.07$ (t, $J=2.6 \mathrm{~Hz}, 1 \mathrm{H}), 1.67-$ $1.53(\mathrm{~m}, 1 \mathrm{H}), 1.54-1.40(\mathrm{~m}, 1 \mathrm{H}), 1.32(\mathrm{~s}, 3 \mathrm{H}), 1.07$ (s, 3H); ${ }^{13} \mathbf{C} \mathbf{N M R}\left(\mathbf{C D C l}_{3}, \mathbf{1 0 1} \mathbf{M H z}\right): \delta$ 175.7, 94.9, 83.5, 78.7, 78.1, 72.4, 61.8, 55.7, 37.7, 29.1, 28.0, 26.1, 25.6, 20.6, 18.4, -5.2, 5.3; HRMS (DART): Calcd for $\mathrm{C}_{19} \mathrm{H}_{35} \mathrm{O}_{4} \mathrm{Si}[\mathrm{M}+\mathrm{H}]^{+}:$353.2143, Found: 353.2140; specific rotation: $[\alpha]_{\mathrm{D}}{ }^{20}=+19.0\left(c 0.29, \mathrm{CHCl}_{3}\right)$.

\subsubsection{2-((3S,3a $R, 6 \mathrm{a} R)-2,2-D i m e t h y l-5-0 x 0-3 a-(p r o p-2-y n-1-y l) h e x a h y d r o f u r o[3,2-$ b] furan-3-yl)acetaldehyde (2)}

In a $250 \mathrm{~mL}$ round-bottom flask containing a stir bar and a solution of alkyne 21 ( $880 \mathrm{mg}, 2.5$ $\mathrm{mmol})$ in THF $(20 \mathrm{~mL})$ was added $(n-\mathrm{Bu})_{4} \mathrm{NF}(1.0 \mathrm{M}, 12.5 \mathrm{~mL}, 12.5 \mathrm{mmol})$ and HOAc $(750$ 
$\mathrm{mg}, 12.5 \mathrm{mmol})$. The mixture was allowed to warm to $40{ }^{\circ} \mathrm{C}$ and stir for $2 \mathrm{~h}$ at $40{ }^{\circ} \mathrm{C}$. The reaction was then quenched by the addition of a saturated solution of aqueous $\mathrm{NH}_{4} \mathrm{Cl}(30 \mathrm{~mL})$ and water $(10 \mathrm{~mL})$. The mixture was washed with EtOAc $(5 \mathrm{x} 40 \mathrm{~mL})$, and the combined organic layers were washed with brine, dried over sodium sulfate and volatiles were removed in vacuo to afford the desired primary alcohol as colorless oil, which was used directly without purification.

In a $500 \mathrm{~mL}$ round-bottom flask containing a stir bar and a solution of the aforementioned alcohol in $\mathrm{CH}_{2} \mathrm{Cl}_{2}(40 \mathrm{~mL})$ cooled to $0{ }^{\circ} \mathrm{C}$, was added $\mathrm{NaHCO}_{3}(840 \mathrm{mg}, 10.0 \mathrm{mmol})$ and DessMartin periodate $(3.18 \mathrm{~g}, 7.5 \mathrm{mmol})$. The mixture was allowed to warm to $22{ }^{\circ} \mathrm{C}$ and stir for 2 $\mathrm{h}$ at $22{ }^{\circ} \mathrm{C}$. The cloudy mixture was filtered through a short pad of celite and washed with $\mathrm{CH}_{2} \mathrm{Cl}_{2}(50 \mathrm{~mL})$ to afford pale-yellow oil, purification of which by silica gel chromatography $\left(10: 1 \rightarrow 3: 1 \rightarrow 3: 2\right.$ hexane:EtOAc; $\mathrm{R}_{f}=0.38(1: 1$ hexanes:EtOAc $\left.=1: 1)\right)$ afforded 2 as white solid (527 mg, $2.22 \mathrm{mmol}, 89 \%$ yield).

White solid; m.p.: $87-89{ }^{\circ} \mathrm{C}, 527 \mathrm{mg}, 2.22 \mathrm{mmol}, 89 \%$ yield; IR (neat): 2972 (w), 2921 (w), 2849 (w), 1773 (s), 1720 (s), 1373 (w), 1266 (m), 1197 (m), 1122 (w), 1180 (w), 1023 (m), 928 (m), $670(\mathrm{w}) \mathrm{cm}^{-1},{ }^{1} \mathbf{H}$ NMR (CDCl, 400 MHz): $\delta 9.78(\mathrm{~s}, 1 \mathrm{H}), 4.51(\mathrm{~d}, J=6.6 \mathrm{~Hz}, 1 \mathrm{H})$, $2.99(\mathrm{dd}, J=18.7,6.7 \mathrm{~Hz}, 1 \mathrm{H}), 2.77(\mathrm{dd}, J=9.1,6.0 \mathrm{~Hz}, 1 \mathrm{H}), 2.74-2.63(\mathrm{~m}, 2 \mathrm{H}), 2.63-2.52$ (m, 1H), $2.48(\mathrm{dd}, J=17.0,2.7 \mathrm{~Hz}, 1 \mathrm{H}), 2.40(\mathrm{dd}, J=16.7,6.1 \mathrm{~Hz}, 1 \mathrm{H}), 2.15-2.08(\mathrm{~m}, 1 \mathrm{H})$, 1.33 (s, 3H), 1.11 (s, 3H); ${ }^{13} \mathbf{C}$ NMR (CDCl 3 , 101MHz): $\delta 199.4,175.0,94.1,83.1,78.7,77.4$, 72.9, 52.7, 40.7, 37.3, 27.9, 25.5, 21.2; HRMS (DART): Calcd for $\mathrm{C}_{13} \mathrm{H}_{17} \mathrm{O}_{4}[\mathrm{M}+\mathrm{H}]^{+}$: 237.1121, Found: 237.1133 ; specific rotation: $[\alpha]_{\mathrm{D}^{20}}=+41.9\left(c 0.70, \mathrm{CHCl}_{3}\right)$.

\section{Previously Reported Characterization Data for Compound $2^{18,23}$}

White solid; m.p.: $93-96{ }^{\circ} \mathrm{C}$, IR (thin film): 2977, 1775, 1722, 1475, 1246, 1199, 1058; ${ }^{\mathbf{1}} \mathbf{H}$ NMR (CDCl 3 , 400 MHz): $\delta 9.81(t, J=2.0 \mathrm{~Hz}, 1 \mathrm{H}), 4.54(\mathrm{~d}, J=6.4 \mathrm{~Hz}, 1 \mathrm{H}), 3.02(\mathrm{dd}, J=$ $18.7,6.7 \mathrm{~Hz}, 1 \mathrm{H}), 2.80(\mathrm{dd}, J=9.0,6.2 \mathrm{~Hz}, 1 \mathrm{H}), 2.73(\mathrm{dd}, J=18.7,0.5 \mathrm{~Hz}, 2 \mathrm{H}), 2.70$ (dd, $J=$ 17.0, $2.6 \mathrm{~Hz}, 1 \mathrm{H}), 2.61$ (ddd, $J=16.7,9.0,2.1 \mathrm{~Hz}, 1 \mathrm{H}), 2.51$ (dd, $J=17.0,2.6 \mathrm{~Hz}, 1 \mathrm{H}), 2.43$ (ddd, J = 16.7, 6.2, 1.9 Hz, 1H), $2.13(\mathrm{t}, J=2.6 \mathrm{~Hz}, 1 \mathrm{H}), 1.37(\mathrm{~s}, 3 \mathrm{H}), 1.14(\mathrm{~s}, 3 \mathrm{H}) ;{ }^{13} \mathbf{C ~ N M R}$ (CDCl 3 , 101 MHz): $\delta$ 199.3, 175.0, 94.1, 83.2, 78.7, 77.4, 72.9, 52.8, 40.7, 37.3, 28.0, 25.5, 21.2; HRMS (ES ${ }^{+}$): Calcd for $\mathrm{C}_{13} \mathrm{H}_{16} \mathrm{O}_{2} \mathrm{Na}[\mathrm{M}+\mathrm{Na}]^{+}:$259.0941, Found: 259.0938; specific rotation: $[\alpha]_{\mathrm{D}}^{25}=+38.5\left(c 1.05, \mathrm{CHCl}_{3}\right)$. 


\subsection{Synthesis of bicyclic fragment of 5-epi-rubriflordilactone $A$ and $B$}

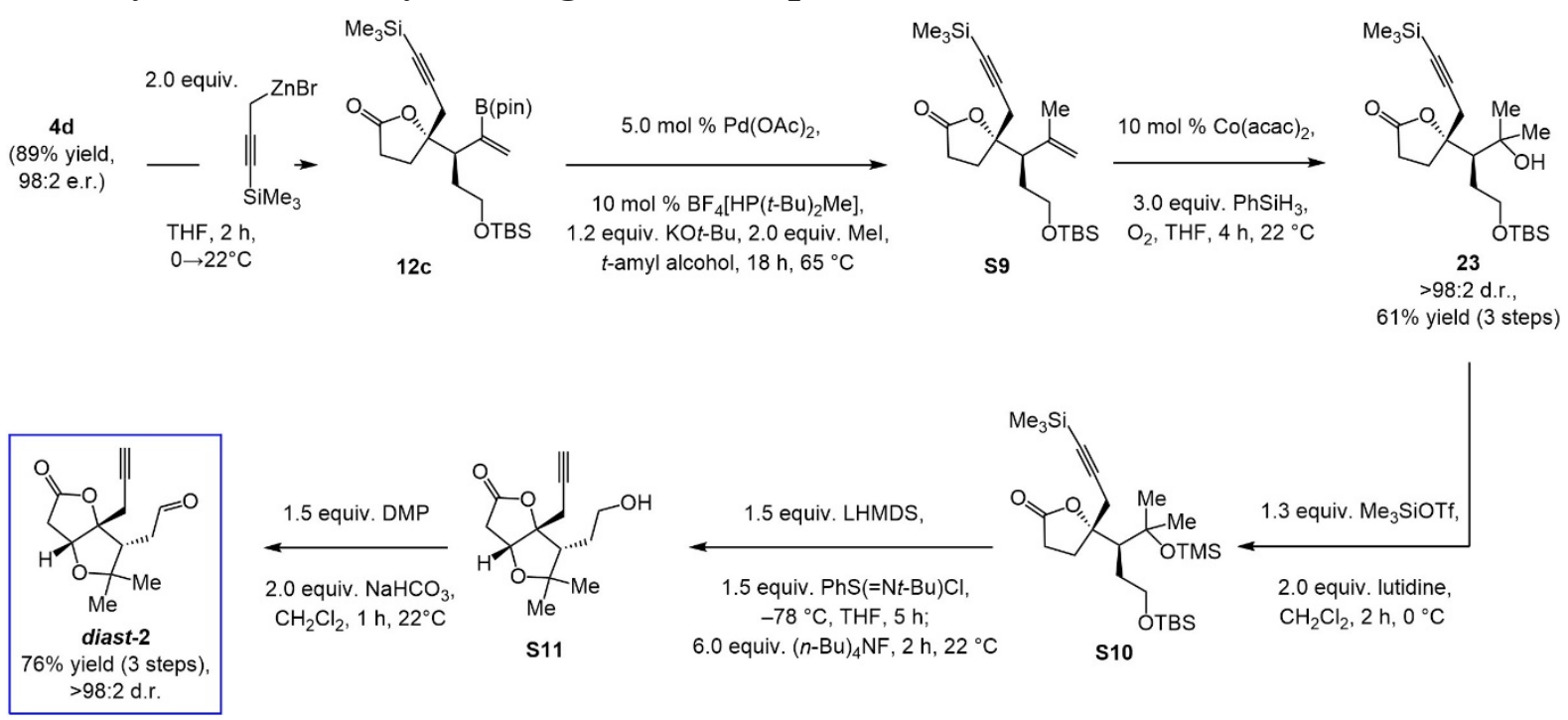

\subsection{1 $(R)-5-((R)-1-((t-B u t y l d i m e t h y l s i l y l) o x y)-4-h y d r o x y-4-m e t h y l p e n t a n-3-y l)-5-(3-$ (trimethylsilyl)prop-2-yn-1-yl)dihydrofuran-2(3H)-one (23)}

A flame-dried two-neck round-bottom flask equipped with a condenser was charged with a suspension of activated $\mathrm{Zn}$ powder $(1.96 \mathrm{~g}, 30.0 \mathrm{mmol})$ in THF $(8.4 \mathrm{~mL})$ and 3-bromo-1trimethylsilyl-1-propyne $(1.62 \mathrm{~mL}, 10.0 \mathrm{mmol})$, and the mixture was allowed to stir at $55^{\circ} \mathrm{C}$ for $15 \mathrm{~min}$ in order to generate $\mathrm{Me}_{3} \mathrm{Si}$-propargyl- $\mathrm{ZnBr}$.

The freshly prepared alkynyl-Zn compound (1.0 M in THF, $4.62 \mathrm{~mL}, 4.62 \mathrm{mmol}$ ) was added at $0{ }^{\circ} \mathrm{C}$ to a solution of ketone $4 \mathbf{d}(725 \mathrm{mg}, 2.31 \mathrm{mmol})$ in THF $(9 \mathrm{~mL})$, and the mixture was allowed to stir for $2 \mathrm{~h}$ while being warmed to $22^{\circ} \mathrm{C}$. The reaction was quenched by the addition an aqueous solution of saturated $\mathrm{NaHCO}_{3}(30 \mathrm{~mL})$, the resulting mixture was washed with $\mathrm{Et}_{2} \mathrm{O}(3 \times 30 \mathrm{~mL})$, and the combined organic layers were dried over $\mathrm{MgSO}_{4}$, filtered and the volatiles were removed in vacuo to afford alkenyl boronate $12 \mathrm{c}$ as colorless oil. This material was used in the next step without purification.

A flame-dried round-bottom flask containing a solution of $12 \mathrm{c}$ in freshly distilled and degassed $t$-amyl alcohol $(23 \mathrm{~mL})$ was charged with $\mathrm{Pd}(\mathrm{OAc})_{2}(26.0 \mathrm{mg}, 116 \mu \mathrm{mol}),[\mathrm{BF} 4]\left[\mathrm{HP}(t-\mathrm{Bu})_{2} \mathrm{Me}\right]$ $(57.3 \mathrm{mg}, 231 \mu \mathrm{mol})$ and $\mathrm{KO} t-\mathrm{Bu}(311 \mathrm{mg}, 2.77 \mathrm{mmol})$. The solution was allowed to stir at $22{ }^{\circ} \mathrm{C}$ for $5 \mathrm{~min}$, after which MeI $(288 \mu \mathrm{L}, 4.62 \mathrm{mmol})$ was added and the mixture was allowed to stir for $18 \mathrm{~h}$ at $65^{\circ} \mathrm{C}$. The solution was subsequently allowed to cool to $22{ }^{\circ} \mathrm{C}$, after which the reaction was quenched by the addition of a saturated solution of aqueous $\mathrm{NaHCO}_{3}(30 \mathrm{~mL})$. The mixture was diluted by the addition of EtOAc $(30 \mathrm{~mL})$, the phases were separated, and the aqueous phase was washed with $\mathrm{Et}_{2} \mathrm{O}(3 \times 5 \mathrm{ml})$. The combined organic layers were dried over $\mathrm{Na}_{2} \mathrm{SO}_{4}$, filtered, and concentrated in vacuo to afford a brown oil/solid. Purification by filtration through a short plug $(6 \mathrm{~cm})$ of silica gel $(10: 1$ petroleum ether:EtOAc) afforded the 1,1-disubstituted alkene $\mathbf{S} 9$ as brown oil (contaminated with 5-10\% unidentified impurities).

In flame-dried round-bottom flask $\mathbf{S 9}$ was dissolved in THF $(40 \mathrm{~mL})$ at $22{ }^{\circ} \mathrm{C}$ and the resulting solution was charged with $\mathrm{PhSiH}_{3}(750 \mathrm{mg}, 855 \mu \mathrm{L}, 6.93 \mathrm{mmol})$ and $\mathrm{Co}(\mathrm{acac})_{2}(59.4 \mathrm{mg}, 0.231$ mmol). Oxygen gas was allowed to pass through the mixture for $4 \mathrm{~h}$, after which the reaction 
was quenched by the addition of a saturated aqueous solution of $\mathrm{NaHCO}_{3}(40 \mathrm{~mL})$. The mixture was washed with EtOAc $(3 \times 40 \mathrm{~mL})$, the combined organic layers were washed with brine $(80$ $\mathrm{mL}$ ), dried over $\mathrm{Na}_{2} \mathrm{SO}_{4}$, filtered, and concentrated under reduced pressure to afford brown oil residue, which was purified by silica gel chromatography $(9: 1 \rightarrow 4: 1$ petroleum ether:EtOAc) to furnish tertiary alcohol 23 as colorless solid (672 $\mathrm{mg}, 1.57 \mathrm{mmol}, 61 \%$ yield for 3 steps). Diastereomeric purity was established by analysis of the ${ }^{1} \mathrm{H}$ NMR spectrum ( $>98: 2$ d.r.).

IR (neat): 3474 (w), 2956 (m), 2929 (w), 2857 (w), 2178 (w), 1760 (s), 1472 (w), 1362 (w), $1250(\mathrm{~m}), 1190$ (m), $1090(\mathrm{~m}), 1007$ (m), $936(\mathrm{w}), 836(\mathrm{~s}), 775(\mathrm{~m}) \mathrm{cm}^{-1} ; \mathrm{R}_{f}=0.21$ (4:1 petroleum ether:EtOAc); ${ }^{1} \mathbf{H}$ NMR (CDCl $\left.\mathbf{3}, \mathbf{4 0 0} \mathbf{M H z}\right): \delta 3.73-3.59(2 \mathrm{H}, \mathrm{m}), 2.94(1 \mathrm{H}, \mathrm{d}, J=$ 17.4), $2.8-2.72(1 \mathrm{H}, \mathrm{m}), 2.74(1 \mathrm{H}, \mathrm{d}, J=17.4 \mathrm{~Hz}), 2.56-2.44(1 \mathrm{H}, \mathrm{m}), 2.47(1 \mathrm{H}, \mathrm{bs}),, 2.45-$ $2.26(2 \mathrm{H}, \mathrm{m}), 1.89(1 \mathrm{H}, \mathrm{dd}, J=5.2,3.1 \mathrm{~Hz}), 1.78(1 \mathrm{H}, \mathrm{dtd}, J=14.8,6.5,5.2 \mathrm{~Hz}), 1.44(1 \mathrm{H}$, $\mathrm{dtd}, J=14.8,6.7,3.1 \mathrm{~Hz}), 1.31(3 \mathrm{H}, \mathrm{s}), 1.26(3 \mathrm{H}, \mathrm{s}), 0.87(9 \mathrm{H}, \mathrm{s}), 0.13(9 \mathrm{H}, \mathrm{s}), 0.04(3 \mathrm{H}, \mathrm{s})$, $0.04(3 \mathrm{H}, \mathrm{s}) ;{ }^{13} \mathbf{C} \mathbf{N M R}\left(\mathbf{C D C l}_{3}, \mathbf{1 0 1} \mathbf{M H z}\right): \delta 176.7,102.3,90.4,88.7,73.0,63.9,53.5,31.1$, 30.8, 30.7, 30.6, 30.1, 29.6, 26.0, 18.4, -0.1, -5.3; HRMS (ESI): Calcd for $\mathrm{C}_{22} \mathrm{H}_{43} \mathrm{O}_{4} \mathrm{Si}_{2}$ $[\mathrm{M}+\mathrm{H}]^{+}: 427.2694$, Found: 427.2682 ; specific rotation: $[\alpha]_{\mathrm{D}}{ }^{20}=+42.3\left(c 1.0, \mathrm{CHCl}_{3}\right)$.

\subsection{2 (3aR,6R,6aR)-6-(2-Hydroxyethyl)-5,5-dimethyl-6a-(prop-2-yn-1- yl)tetrahydrofuro[3,2-b]furan-2(3H)-one (S10)}

In a flame-dried round-bottom flask tertiary alcohol $23(130 \mathrm{mg}, 0.30 \mathrm{mmol})$ was dissolved in $\mathrm{CH}_{2} \mathrm{Cl}_{2}(2 \mathrm{~mL})$, and the mixture was allowed to cool to $0{ }^{\circ} \mathrm{C}$, after which it was charged with 2,6-lutidine $(71 \mu \mathrm{L}, 0.61 \mathrm{mmol})$ and trimethylsilyl triflate (TMSOTf; $72 \mu \mathrm{L}, 0.40 \mathrm{mmol}$ ). The mixture was allowed to stir at $0{ }^{\circ} \mathrm{C}$ for $2 \mathrm{~h}$. The reaction was then quenched by the addition of a saturated solution of aqueous $\mathrm{NaHCO}_{3}(5 \mathrm{~mL})$. The mixture was diluted with $\mathrm{Et}_{2} \mathrm{O}(5 \mathrm{~mL})$, the phases were separated, and the aqueous phase was washed with $\mathrm{Et}_{2} \mathrm{O}(3 \times 4 \mathrm{~mL})$. The combined organic layers were washed with a $10 \%$ aqueous solution of $\mathrm{CuSO}_{4}(10 \mathrm{~mL})$, dried over $\mathrm{MgSO}_{4}$, filtered and the volatiles were removed in vacuo to afford lactone $\mathbf{S 1 0}$ as paleyellow oil. This material was used in the next step without further purification.

To a flame-dried round-bottom flask containing a solution of $\mathbf{S 1 0}$ dissolved in THF $(3 \mathrm{~mL})$ maintained at $-78{ }^{\circ} \mathrm{C}$, was added lithium hexamethyldisilazide (LiHMDS; $1.0 \mathrm{M}$ solution in $\mathrm{THF}, 457 \mu \mathrm{L}, 0.46 \mathrm{mmol})$. The mixture was allowed to stir at $-78{ }^{\circ} \mathrm{C}$ for $1 \mathrm{~h}$ before being charged with a solution of $N$ - $t$-butylbenzenesulfinimidoyl chloride ( $99 \mathrm{mg}, 0.46 \mathrm{mmol}$ ) in THF $(1 \mathrm{~mL})$. The solution was allowed to warm to $22{ }^{\circ} \mathrm{C}$ over a period of $1 \mathrm{~h}$ and then allowed to stir at $22{ }^{\circ} \mathrm{C}$ for $3 \mathrm{~h}$. Next, a solution of $(n-\mathrm{Bu})_{4} \mathrm{NF}(1.0 \mathrm{M}$ in THF, $1.83 \mathrm{~mL}, 1.83 \mathrm{mmol})$ was added and the mixture was allowed to stir at $22{ }^{\circ} \mathrm{C}$ for $2 \mathrm{~h}$. The reaction was then quenched by addition of an aqueous solution of saturated $\mathrm{NaHCO}_{3}(10 \mathrm{~mL})$. The mixture was washed with $\mathrm{CH}_{2} \mathrm{Cl}_{2}(3 \times 20 \mathrm{~mL})$, and the combined organics were dried over $\mathrm{Na}_{2} \mathrm{SO}_{4}$, filtered and concentrated under reduced pressure to afford yellow oil. Purification by silica gel chromatography $\left(\mathrm{R}_{f}=0.14\right.$ in 1:1 petroleum ether:EtOAc; $3: 2 \rightarrow 1: 1$ petroleum ether:EtOAc) afforded bicyclic lactone $\mathbf{S 1 1}$ as colorless oil (59.5 $\mathrm{mg}, 0.25 \mathrm{mmol}, 82 \%$ overall yield). Spectroscopic analysis indicated that a single isomer was generated ( $>98: 2$ d.r.). 
IR (neat): 3396 (w), 2954 (w), 2926 (m), 2856 (w), 2172 (w), 1778 (m), 11378 (w), 1156 (m), 1093 (s), 1029 (m), 936 (m) cm ${ }^{-1} ;{ }^{1} \mathbf{H}$ NMR (CDCl 3 , 400 MHz): $\delta 4.67$ (1H, d, $\left.J=5.8 \mathrm{~Hz}\right)$, 3.86-3.69 (2H, m), 2.96 (1H, dd, $J=18.7,6.5 \mathrm{~Hz}), 2.82(1 \mathrm{H}, \mathrm{dd}, J=17.2,2.7 \mathrm{~Hz}), 2.69-2.50$ $(2 \mathrm{H}, \mathrm{m}), 2.27(1 \mathrm{H}, \mathrm{t}, J=7.1 \mathrm{~Hz}), 2.11(1 \mathrm{H}, \mathrm{t}, J=2.7 \mathrm{~Hz}), 1.90(1 \mathrm{H}, \mathrm{dtd}, J=14.2,7.0,6.2 \mathrm{~Hz})$, $1.72(1 \mathrm{H}, \mathrm{dq}, J=14.2,6.5 \mathrm{~Hz}), 1.69(1 \mathrm{H}, \mathrm{bs}), 1.32(3 \mathrm{H}, \mathrm{s}), 1.16(3 \mathrm{H}, \mathrm{s}) ;{ }^{13} \mathbf{C} \mathbf{~ N M R}\left(\mathbf{C D C l}_{\mathbf{3}}\right.$, $101 \mathrm{MHz}): \delta 175.7,95.5,85.1,79.7,78.2,72.4,60.6,50.9,38.9,30.0,28.5,27.1,24.9$; HRMS (ESI): Calcd for $\mathrm{C}_{13} \mathrm{H}_{22} \mathrm{O}_{4}[\mathrm{M}+\mathrm{H}]^{+}: 238.1278$, Found: 239.1282 ; specific rotation: $[\alpha]_{\mathrm{D}}{ }^{20}=$ $+32.0\left(c 1.0, \mathrm{CHCl}_{3}\right)$.

\subsubsection{2-((3R,3aR,6a $R)-2,2-D i m e t h y l-5-0 x 0-3 a-(p r o p-2-y n-1-y l) h e x a h y d r o f u r o[3,2-$ b]furan-3-yl)acetaldehyde (diast-2)}

To a flame-dried round-bottom flask containing a solution of alcohol $\mathbf{S 1 1}$ (48 $\mathrm{mg}, 0.20 \mathrm{mmol}$ ) in $\mathrm{CH}_{2} \mathrm{Cl}_{2}(2 \mathrm{~mL})$ was added at $22{ }^{\circ} \mathrm{C}$ with Dess-Martin periodinane $(127 \mathrm{mg}, 0.30 \mathrm{mmol})$ and $\mathrm{NaHCO}_{3}(34 \mathrm{mg}, 0.40 \mathrm{mmol})$. The solution was allowed to stir at $22{ }^{\circ} \mathrm{C}$ for $1 \mathrm{~h}$ before the reaction was quenched by the addition of a saturated solution of aqueous $\mathrm{Na}_{2} \mathrm{~S}_{2} \mathrm{O}_{3}(3 \mathrm{~mL})$. The mixture was diluted with a saturated solution of aqueous $\mathrm{NaHCO}_{3}(3 \mathrm{~mL})$ and $\mathrm{Et}_{2} \mathrm{O}(10 \mathrm{~mL})$ and was then allowed to stir vigorously for $10 \mathrm{~min}$. The aqueous phase was washed with $\mathrm{Et}_{2} \mathrm{O}$ $(3 \times 3 \mathrm{~mL})$, after which the combined organic layers were dried over $\mathrm{MgSO}_{4}$, filtered and concentrated under reduced pressure to afford yellow oil residue. Purification by silica gel chromatography $\left(\mathrm{CH}_{2} \mathrm{Cl}_{2}\right)$ delivered aldehyde diast-2 as colorless oil (44 $\mathrm{mg}, 0.19 \mathrm{mmol}, 93 \%$ yield). Note: diast-2 is prone to decomposition and must be stored at $-20{ }^{\circ} \mathrm{C}$ (freezer) and preferably used within $48 \mathrm{~h}$.

IR (neat): 2977 (w), 2917 (m), 2846 (w), 2165 (w), 1785 (s), 1722 (m), 1371 (w), 1199 (m), 1077 (m), 1049 (m), 931 (w) cm ${ }^{-1} ; \mathrm{R}_{f}=0.12\left(100 \% \mathrm{CH}_{2} \mathrm{Cl}_{2}\right) ;{ }^{1} \mathbf{H} \mathbf{N M R}\left(\mathbf{C D C l}_{3}, 400 \mathbf{~ M H z}\right)$ : $\delta 9.85(1 \mathrm{H}, \mathrm{s}), 4.68(1 \mathrm{H}, \mathrm{dd}, J=6.2,0.8 \mathrm{~Hz}), 2.97(1 \mathrm{H}, \mathrm{dd}, J=18.7,6.2 \mathrm{~Hz}), 2.90(1 \mathrm{H}, \mathrm{ddd}, \mathrm{J}$ $=18.7,7.2,0.8 \mathrm{~Hz}, 2 \mathrm{H}), 2.79-2.69(2 \mathrm{H}, \mathrm{m}), 2.69-2.56(3 \mathrm{H}, \mathrm{m}), 2.11(1 \mathrm{H}, \mathrm{t}, J=2.7 \mathrm{~Hz}), 1.32$ $(3 \mathrm{H}, \mathrm{s}), 1.14(3 \mathrm{H}, \mathrm{s}) ;{ }^{13} \mathbf{C}$ NMR (CDCl $\left.3,101 \mathbf{M H z}\right): \delta 199.8,175.1,94.8,84.3,79.4,77.9$, 72.6, 48.2, 40.6, 38.5, 29.3, 27.1, 25.0; HRMS (ESI): Calcd for $\mathrm{C}_{13} \mathrm{H}_{17} \mathrm{O}_{4}[\mathrm{M}+\mathrm{H}]^{+}:$: 237.1121, Found: 237.1118 ; specific rotation: $[\alpha]_{\mathrm{D}}{ }^{20}=+50.4\left(c 1.0, \mathrm{CHCl}_{3}\right)$.

\section{Determination of the absolute and relative configuration of products}

Case 1: The absolute and relative configuration of products was ascertained by X-ray structure of ketone $\mathbf{S 1 2}$, which was obtained by oxidation of $(\boldsymbol{S}, \boldsymbol{R})-\mathbf{8 d}\left(\mathrm{NaBO}_{3} \cdot 4 \mathrm{H}_{2} \mathrm{O}\right)$. Suitable single crystals were obtained through slow evaporation of a hexanes solution. The X-ray structure reveals that the organocerium addition affords the erythro isomer (anti orientation of two stereogenic centers), with $S$ configuration for the stereogenic center at the tertiary carbon and the quaternary carbon being of $S$ configuration. (Although the stereochemistry is the same for compound $(\boldsymbol{S}, \boldsymbol{R})-\mathbf{8 d}$ and for $\mathbf{S 3}$; that is, the substituents are assigned with opposite priorities). 

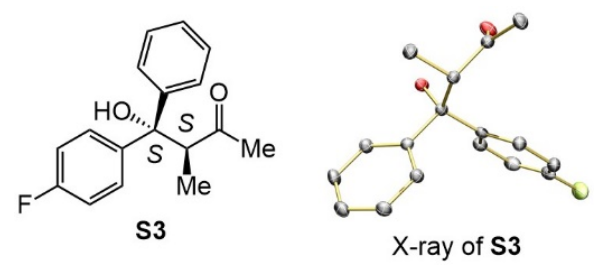

Case 2: The stereochemical identity of allyl-substituted tertiary alcohols was confirmed independently from other organometallic additions. The relative stereochemistry of allylsubstituted tertiary alcohol 9a (see Scheme 10) was established by analysis of data from a NOESY experiment that was performed on acetonide S12 (Scheme S5).

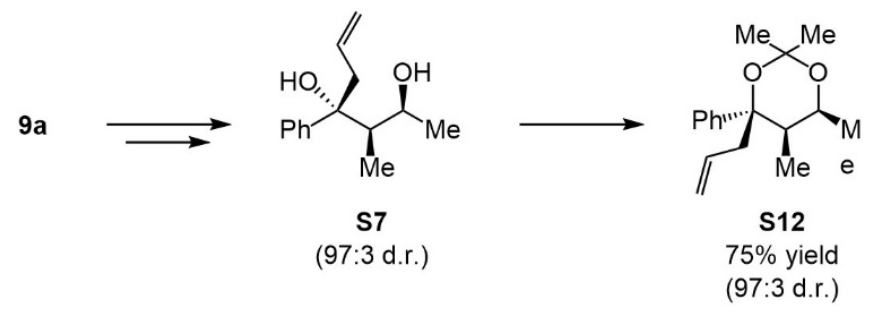

Scheme S5. Preparation of acetonide S13 in three steps from 9a.

\subsection{1 (4R,5R,6S)-4-allyl-2,2,5,6-tetramethyl-4-phenyl-1,3-dioxane (S12)}

An oven-dried vial was charged with 1,3-diol S7 (34.0 $\mathrm{mg}, 0.1540 \mathrm{mmol}$ ) and 2,2dimethoxypropane $(2.0 \mathrm{~mL})$. To this solution was added acetone $(1 \mathrm{~mL})$ and pyridinium $p$ toluene sulfonate $(3.9 \mathrm{mg}, 0.0155 \mathrm{mmol})$. The mixture was allowed to reflux for $1.5 \mathrm{~h}$, until full conversion was observed by TLC analysis. Removal of solvents in vacuo afforded colorless oil, which was purified by silica gel chromatography (40:1 hexanes/ $\left.\mathrm{Et}_{2} \mathrm{O}\right)$.

Colorless oil; $30.0 \mathrm{mg}, 0.1155 \mathrm{mmol}, 75 \%$ yield, $97: 3$ d.r. determined by ${ }^{1} \mathrm{H}$ NMR in $\mathrm{CDCl}_{3}$; IR (neat): 2981 (m), 2935 (m), 2917 (w), 1452 (m), 1380 (m), 1253 (m), 1021 (s), 705 (s)

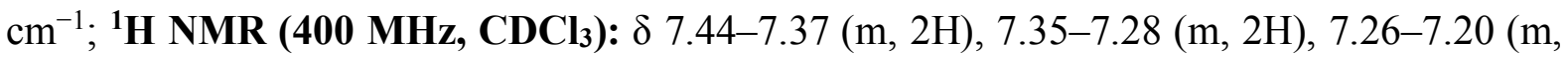
$1 \mathrm{H}), 5.38-5.08(\mathrm{~m}, 1 \mathrm{H}), 4.91-4.69(\mathrm{~m}, 2 \mathrm{H}), 4.18(\mathrm{qd}, \mathrm{J}=6.4,1.7 \mathrm{~Hz}, 1 \mathrm{H}), 2.67(\mathrm{dd}, \mathrm{J}=13.7$, $8.4 \mathrm{~Hz}, 1 \mathrm{H}), 2.35(\mathrm{dd}, \mathrm{J}=13.7,6.0 \mathrm{~Hz}, 1 \mathrm{H}), 2.15(\mathrm{qd}, \mathrm{J}=6.7,1.7 \mathrm{~Hz}, 1 \mathrm{H}), 1.44(\mathrm{~s}, 3 \mathrm{H}), 1.18$ $(\mathrm{d}, \mathrm{J}=6.4 \mathrm{~Hz}, 3 \mathrm{H}), 1.14$ (d, J = 6.7 Hz, 3H), 0.90 (s, 3H). ${ }^{13} \mathbf{C}$ NMR (101 MHz, CDCl $)$ : 145.2, 132.2 , 127.8, 127.5, 126.9, 118.28, 100.0, 79.8, 65.0, 47.9, 33.5, 31.5, 25.2, 19.4, 7.0. HRMS (DART): Calcd for $\mathrm{C}_{17} \mathrm{H}_{24} \mathrm{O}_{2}[\mathrm{M}+\mathrm{H}]^{+}: 260.1776$, Found: 260.1778 ; specific rotation: $[\alpha]_{\mathrm{D}}{ }^{20}$ $=-41.3\left(c\right.$ 1.0, $\left.\mathrm{CHCl}_{3}\right)$ for an enantiomerically enriched sample of 97:3 e.r.

Based on the NOESY experiment (Scheme S6), the stereogenic center was determined to have $R$ configuration. 


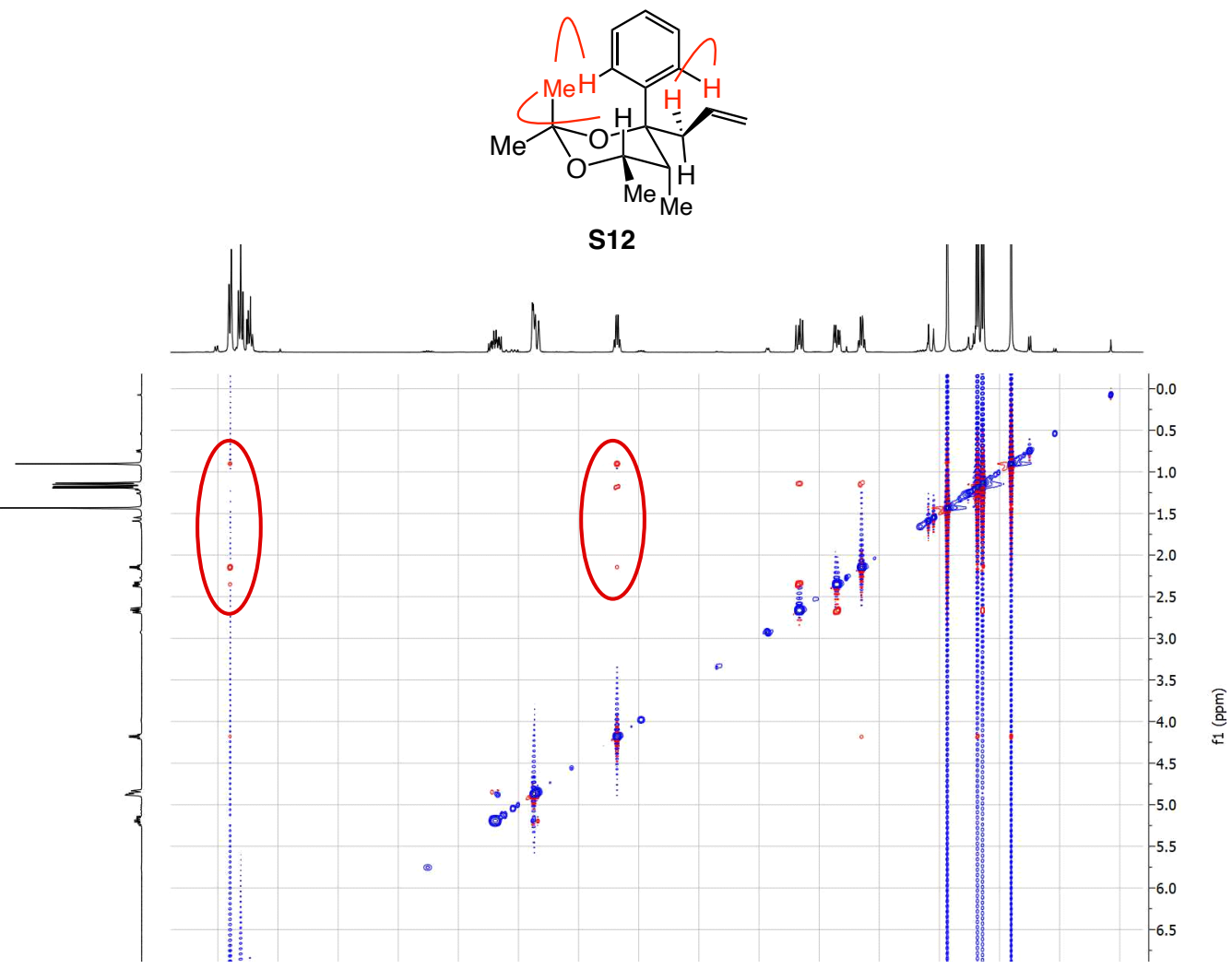

Scheme S6. NOESY experiment to determine the relative stereochemistry of acetonide S12; key enhancements are highlighted.

Case 3: See the analytical data for tertiary homoallylic alcohol 19, below.

Case 4: The absolute configuration of $\beta$-boryl ketones generated from reactions involving disubstituted allenes was established based on X-ray structure of $\mathbf{5 h}$. Suitable single crystals were obtained by slow evaporation of hexanes solution. The stereogenic center was thus found to be of $R$ configuration. Additions to ketones are expected to furnish the same stereochemical outcome as those bearing tertiary stereogenic centers, according to the Felkin-Anh model.

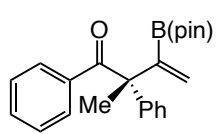

$5 \mathrm{~g}$

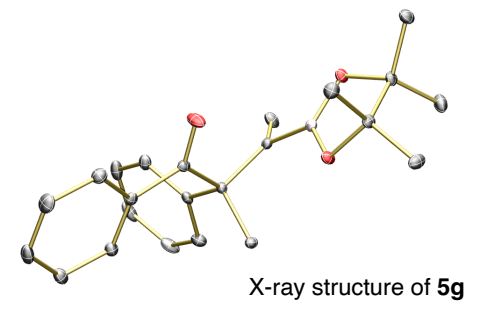

\section{X-ray Structures}

X-ray intensity data were measured at 100(2) K (Oxford Cryostream 700) on a Bruker Kappa APEX Duo diffractometer system equipped with a sealed Mo-target X-ray tube $(\lambda=0.71073$ $\AA)$ and a high brightness $\mathrm{I} \mu \mathrm{S}$ copper source $(\lambda=1.54178 \AA$ ). The crystals were mounted on a goniometer head with paratone oil. The detector was placed at a distance of 5.000 or $6.000 \mathrm{~cm}$ from the crystal. For each experiment, data collection strategy was determined by APEX 
software package and all frames were collected with a scan width of $0.5^{\circ}$ in $\omega$ and $\phi$ with an exposure time of 10 or $20 \mathrm{~s} /$ frame.

The frames were integrated with the Bruker SAINT Software package using a narrow- frame integration algorithm to a maximum $2 \theta$ angle of $56.54^{\circ}(0.75 \AA$ resolution $)$ for Mo data and of $134^{\circ}$ ( $0.84 \AA$ resolution) for $\mathrm{Cu}$ data. The final cell constants are based upon the refinement of the XYZ-centroids of several thousand reflections above $20 \sigma(\mathrm{I})$. Analysis of the data showed negligible decay during data collection. Data were corrected for absorption effects using the empirical method (SADABS). The structures were solved and refined by full-matrix least squares procedures on $\left|F^{2}\right|$ through the use of the Bruker SHELXTL (version 6.12) software package. All hydrogen atoms were included in idealized positions for structure factor calculations except for those forming hydrogen bonds or on a chiral center. Anisotropic displacement parameters were assigned to all non-hydrogen atoms, except those disordered.

\subsection{X-ray structure of $r a c-2 \mathrm{j}$}

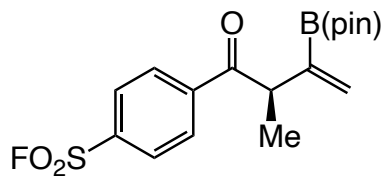

2j

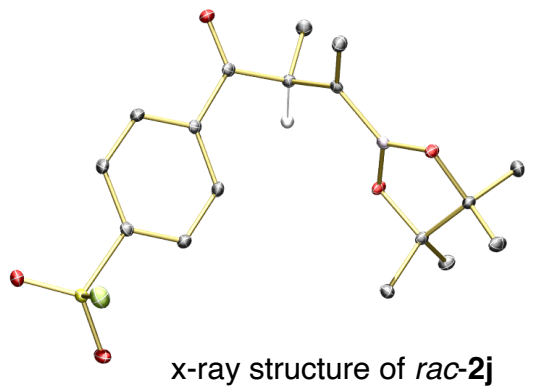

$\mathrm{x}$-ray structure of rac-2j

Table S10. Crystal data and structure refinement for $r a c-2 j$

Identification code

Empirical formula

Formula weight

Temperature

Wavelength

Crystal system

Space group

Unit cell dimensions

Volume

Z

Density (calculated) $r a c-2 \mathbf{j}$

C17 H22 B F O5 S

368.21

100(2) K

$1.54178 \AA$

Triclinic

P-1

$$
\begin{array}{ll}
\mathrm{a}=6.1308(3) \AA & \mathrm{a}=105.1340(10)^{\circ} . \\
\mathrm{b}=9.7304(5) \AA & \mathrm{b}=96.515(2)^{\circ} . \\
\mathrm{c}=16.5174(9) \AA & \mathrm{g}=100.997(2)^{\circ} .
\end{array}
$$

919.74(8) $\AA^{3}$

2

$1.330 \mathrm{Mg} / \mathrm{m}^{3}$ 
Absorption coefficient

$\mathrm{F}(000)$

Crystal size

Theta range for data collection

Index ranges

Reflections collected

Independent reflections

Completeness to theta $=66.695 \infty 0$

Absorption correction

Max. and min. transmission

Refinement method

Data / restraints / parameters

Goodness-of-fit on $\mathrm{F}^{2}$

Final R indices [I $>2 \operatorname{sigma}(\mathrm{I})]$

$\mathrm{R}$ indices (all data)

Extinction coefficient

Largest diff. peak and hole
$1.864 \mathrm{~mm}^{-1}$

388

$0.500 \times 0.250 \times 0.200 \mathrm{~mm}^{3}$

4.837 to $66.695^{\circ}$.

$-7<=\mathrm{h}<=7,-11<=\mathrm{k}<=11,-19<=\mathrm{l}<=19$

11619

$3227[\mathrm{R}(\mathrm{int})=0.0239]$

$98.9 \%$

Semi-empirical from equivalents

0.7528 and 0.6030

Full-matrix least-squares on $\mathrm{F}^{2}$

3227 / 0 / 231

1.046

$\mathrm{R} 1=0.0326, \mathrm{wR} 2=0.0886$

$\mathrm{R} 1=0.0328, \mathrm{wR} 2=0.0888$

$\mathrm{n} / \mathrm{a}$

0.483 and -0.349 e. $\AA^{-3}$ 


\subsection{X-ray Crystal Structure of $R-5 \mathrm{~g}$}

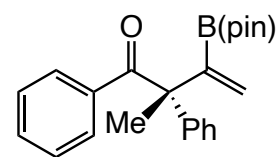

$5 \mathrm{~g}$

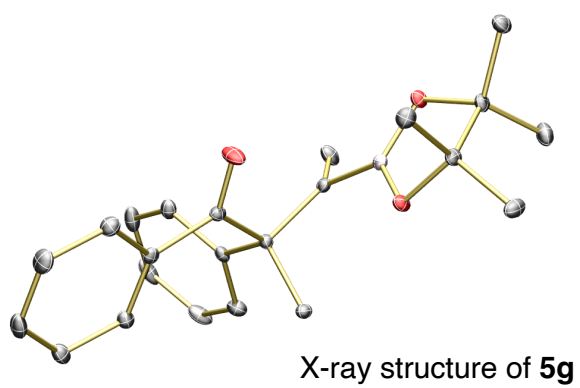

X-ray structure of $\mathbf{5 g}$

Table S11. Crystal data and structure refinement for ketone $5 \mathrm{~g}$

Identification code

Empirical formula

Formula weight

Temperature

Wavelength

Crystal system

Space group

Unit cell dimensions

Volume

Z

Density (calculated)

Absorption coefficient

$\mathrm{F}(000)$

Crystal size

Theta range for data collection

Index ranges

Reflections collected

Independent reflections

Completeness to theta $=66.638 \infty 0$

Absorption correction
$R-5 g$

C23 H27 B O3

362.25

100(2) K

$1.54178 \AA$

Orthorhombic

$\mathrm{P} 22_{1} 2_{1}$

$$
\begin{array}{ll}
\mathrm{a}=6.2313(2) \AA & \mathrm{a}=90^{\circ} . \\
\mathrm{b}=15.8456(4) \AA & \mathrm{b}=90^{\circ} . \\
\mathrm{c}=20.2341(5) \AA & \mathrm{g}=90^{\circ} .
\end{array}
$$

1997.89(10) $\AA^{3}$

4

$1.204 \mathrm{Mg} / \mathrm{m}^{3}$

$0.608 \mathrm{~mm}^{-1}$

776

$0.460 \times 0.120 \times 0.080 \mathrm{~mm}^{3}$

3.543 to $66.638^{\circ}$.

$-7<=\mathrm{h}<=7,-18<=\mathrm{k}<=18,-23<=1<=24$

24460

$3535[\mathrm{R}(\mathrm{int})=0.0547]$

$100.0 \%$

Semi-empirical from equivalents 
Max. and min. transmission

Refinement method

Data / restraints / parameters

Goodness-of-fit on $\mathrm{F}^{2}$

Final $\mathrm{R}$ indices $[\mathrm{I}>2 \operatorname{sigma}(\mathrm{I})]$

$\mathrm{R}$ indices (all data)

Absolute structure parameter

Extinction coefficient

Largest diff. peak and hole
0.7528 and 0.6667

Full-matrix least-squares on $\mathrm{F}^{2}$

3535 / 0 / 249

1.032

$\mathrm{R} 1=0.0263, \mathrm{wR} 2=0.0655$

$\mathrm{R} 1=0.0277, \mathrm{wR} 2=0.0666$

$0.03(7)$

$\mathrm{n} / \mathrm{a}$

0.149 and -0.141 e. $\AA^{-3}$ 


\subsection{X-ray Crystal Structure of $(S, S)-S 3$}

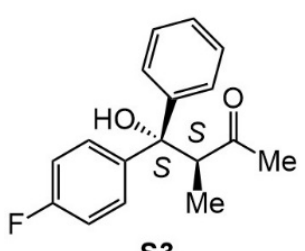

S3

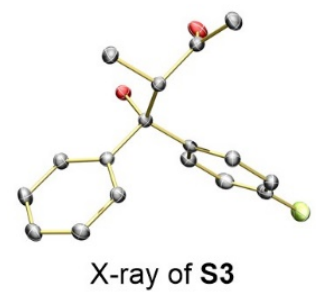

X-ray of S3

Table S12. Crystal data and structure refinement for Alcohol S3

Identification code

Empirical formula

Formula weight

Temperature

Wavelength

Crystal system

Space group

Unit cell dimensions

Volume

Z

Density (calculated)

Absorption coefficient

$\mathrm{F}(000)$

Crystal size

Theta range for data collection

Index ranges

Reflections collected

Independent reflections

Completeness to theta $=66.351 \infty$

Absorption correction

Max. and min. transmission
$(3 S, 4 S)-\mathrm{S3}$

C17 H17 F O2

272.30

173(2) K

$1.54178 \AA$

Orthorhombic

$\mathrm{P} 2{ }_{1} 2{ }_{1}{ }_{1}$

$$
\begin{array}{ll}
\mathrm{a}=8.3512(5) \AA & \mathrm{a}=90^{\circ} . \\
\mathrm{b}=12.8409(8) \AA & \mathrm{b}=90^{\circ} . \\
\mathrm{c}=13.0292(8) \AA & \mathrm{g}=90^{\circ} .
\end{array}
$$

1397.21(15) $\AA 3$

4

$1.295 \mathrm{Mg} / \mathrm{m}^{3}$

$0.757 \mathrm{~mm}^{-1}$

576

$0.580 \times 0.190 \times 0.160 \mathrm{~mm}^{3}$

4.835 to $66.351^{\circ}$.

$-9<=\mathrm{h}<=9,-13<=\mathrm{k}<=15,-15<=\mathrm{l}<=15$

9783

$2405[\mathrm{R}(\mathrm{int})=0.0250]$

$98.8 \%$

Semi-empirical from equivalents

0.7528 and 0.6282 
Refinement method

Data / restraints / parameters

Goodness-of-fit on $\mathrm{F}^{2}$

Final $\mathrm{R}$ indices [I $>2 \operatorname{sigma}(\mathrm{I})]$

$\mathrm{R}$ indices (all data)

Absolute structure parameter

Extinction coefficient

Largest diff. peak and hole
Full-matrix least-squares on $\mathrm{F}^{2}$

$2405 / 1 / 186$

1.079

$\mathrm{R} 1=0.0272, \mathrm{wR} 2=0.0718$

$\mathrm{R} 1=0.0281, \mathrm{wR} 2=0.0727$

$0.06(5)$

$\mathrm{n} / \mathrm{a}$

0.115 and -0.141 e. $\AA-3$

\subsection{X-ray Crystal Structure of 2}

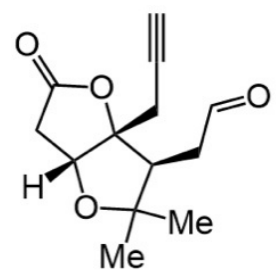

2

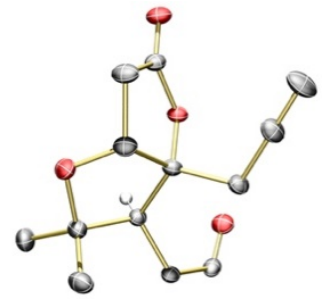

X-Ray of 2

Table S13. Crystal data and structure refinement for 2

Identification code

Empirical formula

Formula weight

Temperature

Wavelength

Crystal system

Space group

Unit cell dimensions

Volume

Z

Density (calculated)

Absorption coefficient
2

C13 H16 O4

236.26

173(2) K

$1.54178 \AA$

Orthorhombic

$\mathrm{P} 2{ }_{1}{ }_{1} 2_{1}$

$$
\begin{array}{ll}
\mathrm{a}=7.6268(3) \AA & \mathrm{a}=90^{\circ} . \\
\mathrm{b}=10.5686(5) \AA & \mathrm{b}=90^{\circ} . \\
\mathrm{c}=15.5705(7) \AA & \mathrm{g}=90^{\circ} .
\end{array}
$$

$1255.05(10) \AA^{3}$

4

$1.250 \mathrm{Mg} / \mathrm{m}^{3}$

$0.763 \mathrm{~mm}^{-1}$ 
$\mathrm{F}(000)$

Crystal size

Theta range for data collection

Index ranges

Reflections collected

Independent reflections

Completeness to theta $=66.604^{\circ}$

Absorption correction

Max. and min. transmission

Refinement method

Data / restraints / parameters

Goodness-of-fit on $\mathrm{F}^{2}$

Final $R$ indices $[\mathrm{I}>2 \operatorname{sigma}(\mathrm{I})]$

$\mathrm{R}$ indices (all data)

Absolute structure parameter

Extinction coefficient

Largest diff. peak and hole
504

$0.560 \times 0.280 \times 0.100 \mathrm{~mm}^{3}$

5.058 to $66.604^{\circ}$.

$-9<=\mathrm{h}<=8,-12<=\mathrm{k}<=12,-18<=\mathrm{l}<=18$

11525

$2161[\mathrm{R}(\mathrm{int})=0.0225]$

$98.2 \%$

Semi-empirical from equivalents

0.7528 and 0.6552

Full-matrix least-squares on $\mathrm{F}^{2}$

$2161 / 0 / 154$

1.062

$\mathrm{R} 1=0.0280, \mathrm{wR} 2=0.0744$

$\mathrm{R} 1=0.0285, \mathrm{wR} 2=0.0751$

$0.04(5)$

$\mathrm{n} / \mathrm{a}$

0.176 and -0.161 e. $\AA^{-3}$

\section{References}

1. Luo, L.; Wilhelm, C.; Sun, A.; Grey, C. P.; Lauher, J. W.; Goroff, N. S. Poly(diiododiacetylene): preparation, isolation, and full characterization of a very simple poly(diacetylene). J. Am. Chem. Soc. 2008, 130, 7702-7709. 2. Eckenberg, P.; Groth, U.; Kohler, T. Stereoselective synthesis of steroids and related compounds, IV. Addition of cerium reagents derived from (trimethylsilyl)propargyl bromide to aldehydes. Liebigs Ann. Chem. 1994, 7, 673-677.

3. Alcaraz, C.; Groth, U. Ligand effects in diastereoselective additions of organocerium reagents to carbonyl substrates. Angew. Chem., Int. Ed. 1997, 36, 2480-2482.

4. Gulliver, D. J.; Levason, W.; Webster, M. Coordination stabilised copper(I) fluoride. Crystal and molecular structure of fluorotris(triphenylphosphine)copper(I) Ethanol (1/2), $\mathrm{Cu}\left(\mathrm{PPh}_{3}\right)_{3} \mathrm{~F} \cdot 2 \mathrm{EtOH}$. Inorganica Chim. Acta 1981, 52, 153-159.

5. Gilbert, J. C.; Weerasooriya, U. Elaboration of aldehydes and ketones to alkynes: improved methodology. $J$. Org. Chem. 1979, 44, 4997-4998. 
6. Kamal, A.; Arifuddin, M.; Venugopal Rao, N. Improved efficient conversion of aldehydes to nitriles via their N, N-dimethyl hydrazones. Synth. Commun. 1998, 28, 4507-4512.

7. Hill, R. R.; Rychnovsky, S. D. Generation, stability, and utility of lithium 4,4'-Di-tert-butylbiphenylide (LiDBB). J. Org. Chem. 2016, 81, 10707-10714.

8. Reetz, M. Y.; Haning, H.; Stanchev, S. Ligand effects in selective carbonyl addition reactions of organomanganese and cerium reagents. Tetrahedron Lett. 1992, 33, 6963-6966.

9. Tsuda, T.; Yazawa, T.; Watanabe, K.; Fujii, T.; Saegusa, T. Preparation of thermally stable and soluble mesitylcopper(I) and its application in organic synthesis. J. Org. Chem. 1981, 46, 192-194.

10. Stollenz, M.; Meyer, F. Mesitylcopper - a powerful tool in synthetic chemistry. Organometallics. 2012, 31, 7708-7727.

11. Lal; S.; Diez-Gonzalez, S. [CuBr( $\left.\left(\mathrm{PPh}_{3}\right)_{3}\right]$ for azide-alkyne cycloaddition reactions under strict click conditions. J. Org. Chem. 2011, 76, 2367-2373.

12. Terao, Y.; Miyamoto, K.; Ohta, H. Introduction of single mutation changes arylmalonate decarboxylase to racemase. Chem. Commun. 2006, 3600-3602.

13. Mu, Y.; Nguyen, T.; Koh, M. J.; Schrock, R. R.; Hoveyda, A. H. E- and Z-, di- and tri-substituted alkenyl nitriles through catalytic cross-metathesis. Nat. Chem. 2019, 11, 478-487.

14. Murray, R. E.; Zweifel, G. Preparation of phenyl cyanate and its utilization for the synthesis of $\alpha, \beta$-unsaturated nitriles. 1980, Synthesis 150.

15. Crabbé, P.; Fillion, H.; André, D.; Luche, J. L. Efficient homologation of acetylenes to allenes. J. Chem. Soc. Chem. Commun. 1979, 859-860.

16. Crandall, J. K.; Batal, D. J.; Lin, F.; Reix, T.; Nadol, G. S.; Ng, R. A. Allene epoxidation. Highly functionalized tetrahydrofurans and tetrahydropyrans from the oxidative cyclization of allenic alcohols. Tetrahedron, 1992, 48, $1427-1448$.

17. Kuang, J.; Ma, S. An efficient synthesis of terminal allenes from terminal 1-alkynes. J. Org. Chem. 2009, 74, $1763-1765$.

18. Zhang, S.; del Pozo, J.; Romiti, F.; Mu, Y.; Torker, S.; Hoveyda A. H. Delayed catalyst function enables direct enantioselective conversion of nitriles to $\mathrm{NH}_{2}$-amines. Science 2019, 364, 45-51.

19. Morrison, R. J.; van der Mei, F. W.; Romiti, F.; Hoveyda, A. H. A catalytic approach for enantioselective synthesis of homoallylic alcohols bearing a $Z$-alkenyl chloride or trifluoromethyl group. A concise and protecting group-free synthesis of mycothiazole. J. Am. Chem. Soc. 2020, 142, 436-447.

20. Qiao, C.; Jeon, H.-B.; Sayre, L. M. Selective inhibition of bovine plasma amine oxidase by homopropargylamine, a new unactivator motif. J. Am. Chem. Soc. 2004, 126, 8038-8045.

21. Bolte, B.; Odabachian, Y.; Gagosz, F. Gold(I)-catalyzed rearrangement of propargyl benzyl ethers: a practical method for the generation and in situ transformation of substituted allenes. J. Am. Chem. Soc. 2010, 132, 72947296.

22. Jung, B.; Hoveyda, A. H. Site- and enantioselective formation of allene-bearing tertiary or quaternary carbon stereogenic centers through NHC-Cu-catalyzed allylic substitution. J. Am. Chem. Soc. 2012, 134, 1490-1493. 
23. Takashi, K.; Fukuyama, T.; Ryu, I. Regioselective radical bromoallylation of allenes leading to 2-bromosubstituted 1,5-dienes. Org. Lett. 2011, 13, 3864-3867.

24. Riveiros, R.; Rodriguez, D.; Perez Sestelo, J.; Sarandeses, L. A. Palladium-catalyzed cross-coupling reaction of triorganoindium reagents with propargylic esters. Org. Lett. 2006, 8, 1403-1406.

25. Moran, J.; Preetz, A.; Mesch, R. A.; Krische. M. J. Iridium-catalysed direct C-C coupling of methanol and allenes. Nature Chem. 2011, 3, 287-290.

26. Trost, B.; Xie, J. Palladium-catalyzed diastereo- and enantioselective Wagner-Meerwein shift: control of absolute stereochemistry in the $\mathrm{C}-\mathrm{C}$ bond migration event. J. Am. Chem. Soc. 2008, 130, 6231-6242.

27. Stergiades, I. A.; Tius, M. A. $\alpha, \beta$-Unsaturated acyl silanes. J. Org. Chem. 1999, 64, 7547-7551.

28. Cainelli, G.; Galletti, P.; Giacomin, D. Solvent effects on stereoselectivity: more than just an environment. Chem.Soc. Rev. 2009, 38, 990-1001.

29. Krasovskiy, A.; Kopp, F.; Knochel, P. Soluble lanthanide salts $\left(\mathrm{LnCl}_{3} \cdot 2 \mathrm{LiCl}\right)$ for the improved addition of organomagnesium reagents to carbonyl compounds. Angew. Chem. Int. Ed. 2006, 45, 497-500.

30. Reich, H. J. Role of organolithium aggregates and mixed aggregates in organolithium mechanisms. Chem. Rev. 2013, 113, 7130-7178.

31. Lovinger, G. J.; Aparece, M. D.; Morken, J. P. Pd-catalyzed conjunctive cross-coupling between Grignardderived boron "ate" complexes and $\mathrm{C}\left(\mathrm{sp}^{2}\right)$ halides or triflates: NaOTf as a Grignard activator and halide scavenger. J. Am. Chem. Soc. 2017, 139, 3153-3160.

32. Read, J. A.; Yang, Y.; Woerpel, K. A. Additions of Organomagnesium Halides to $\alpha$-Alkoxy Ketones: Revision of the Chelation-Control Model. Org. Lett. 2017, 19, 3346-3349.

33. Krasovskiy, A.; Knochel, P. A LiCl-mediated $\mathrm{Br} / \mathrm{Mg}$ exchange reaction for the preparation of functionalized aryl- and heteroarylmagnesium compounds from organic bromides. Angew. Chem. Int. Ed. 2004, 43, 3333-3336. 34. Jang, H.; Romiti, F.; Torker, S.; Hoveyda, A. H. Catalytic diastereo- and enantioselective additions of versatile allyl groups to N-H ketimines. Nat. Chem. 2017, 9, 1269-1275.

35. Evans, D. A.; Chapman, K. T.; Carreira, E. M. Directed reduction of $\beta$-hydroxy ketones employing tetramethylammonium triacetoxyborohydride. J. Am. Chem. Soc. 1988, 110, 3560-3578. 